\title{
MicroRNAs in the heart : micromanagers and sentinels of cardiac disease
}

Citation for published version (APA):

Corsten, M. F. (2012). MicroRNAs in the heart : micromanagers and sentinels of cardiac disease.

[Doctoral Thesis, Maastricht University]. Maastricht University. https://doi.org/10.26481/dis.20120914mc

Document status and date:

Published: 01/01/2012

DOI:

10.26481/dis.20120914mc

Document Version:

Publisher's PDF, also known as Version of record

\section{Please check the document version of this publication:}

- A submitted manuscript is the version of the article upon submission and before peer-review. There can be important differences between the submitted version and the official published version of record.

People interested in the research are advised to contact the author for the final version of the publication, or visit the DOI to the publisher's website.

- The final author version and the galley proof are versions of the publication after peer review.

- The final published version features the final layout of the paper including the volume, issue and page numbers.

Link to publication

\footnotetext{
General rights rights.

- You may freely distribute the URL identifying the publication in the public portal. please follow below link for the End User Agreement:

www.umlib.nl/taverne-license

Take down policy

If you believe that this document breaches copyright please contact us at:

repository@maastrichtuniversity.nl

providing details and we will investigate your claim.
}

Copyright and moral rights for the publications made accessible in the public portal are retained by the authors and/or other copyright owners and it is a condition of accessing publications that users recognise and abide by the legal requirements associated with these

- Users may download and print one copy of any publication from the public portal for the purpose of private study or research.

- You may not further distribute the material or use it for any profit-making activity or commercial gain

If the publication is distributed under the terms of Article $25 \mathrm{fa}$ of the Dutch Copyright Act, indicated by the "Taverne" license above, 
MicroRNAs in the Heart Micromanagers and sentinels of cardiac disease 
The research described in this thesis was supported by a grant of the Dutch Heart Foundation (DHF-2007B036). Also, financial support for the publication of this thesis by the Dutch Heart Foundation and the Rescar Hartsvrienden Foundation Maastricht is gratefully acknowledged.

ISBN: 9789461083340

Vormgeving en lay-out: wenziD.nl, Wendy Schoneveld

Printed: Gildeprint Drukkerijen

Copyright (c) 2012 Maarten F. Corsten 


\section{MicroRNAs in the Heart}

Micromanagers and sentinels of cardiac disease

\section{PROEFSCHRIFT}

ter verkrijging van de graad van doctor aan de Universiteit Maastricht op gezag van de Rector Magnificus, Prof. dr. L.L.G. Soete, volgens het besluit van het College van Decanen in het openbaar te verdedigen op vrijdag 14 september 2012 om 12.00 uur

door

\section{Martinus Franciscus Corsten}




\section{Promotor}

Prof. dr. S. Heymans

\section{Copromotor}

Dr. B. Schroen

\section{Beoordelingscommissie}

Prof. dr. L. de Windt (voorzitter)

Prof. dr. H.J.G.M. Crijns

Prof. dr. M.J. Post

Prof. dr. Y.M. Pinto, Academic Medical Center, Amsterdam, Nederland

Prof. dr. T. Thum, Hannover Medical School, Duitsland 


\section{Contents}

Chapter 1 | General Introduction

Chapter $2 \mid$ MicroRNA Biology

Chapter $3 \mid$ Inflammation in Viral Myocarditis: Friend or Foe?

Chapter 4 MicroRNA Profiling Identifies MicroRNA-155 as an Adverse Mediator of Cardiac Injury and Dysfunction during Acute Viral Myocarditis

Chapter 5| The MicroRNA-221-222 Family Controls Viral Replication and Cardiac Inflammation in Acute Enteroviral Myocarditis

Chapter $6 \mid$ Macrophage MicroRNA-155 Controls Cardiac Hypertrophy and Failure

Chapter 7 Circulating MicroRNA-208b and MicroRNA-499 Reflect Myocardial Damage in Cardiovascular Disease

Chapter $8 \mid$ General Discussion

Summary/Samenvatting

Nawoord

Curriculum Vitae

214

List of Publications

215 

General Introduction 
Heart failure is a grave cardiac condition that affects approximately 15 million people in Europe alone and has a four-year mortality of $40 \%$ after the first hospital admission ${ }^{1,2}$. The clinical syndrome of heart failure occurs when the capacity of the heart to distribute oxygenand nutrient-rich blood to bodily cells is no longer capable of meeting peripheral demands. It is the common final pathway of different cardiovascular disorders, including myocardial infarction, valvular heart disease, hypertension, rhythm disturbances, and genetic, viral, toxic and autoimmune cardiomyopathies ${ }^{2}$. The significance of altered hemodynamics and neurohumoral signaling in patients with heart failure has long been recognized. In addition, the emergence of molecular biology in cardiovascular research in the nineties has provided important insights into the intracellular events that accompany these alterations. Cellular functions are governed by the dynamic expression of genes in our DNA. Such genes encode for proteins with a wide variety of cellular functions, such as cell structure reinforcement, energy production, intercellular communication and the regulation of gene expression. In heart failure, cardiac cells - including cardiomyocytes, fibroblasts, endothelial cells, vascular smooth muscle cells, and resident macrophages or dendritic cells - respond to altered environmental demands by activation of gene expression networks that can be adaptive or maladaptive ${ }^{3}$. An important research goal in molecular cardiology is to identify such adaptive or maladaptive gene programs involved in heart diseases, and design targeted therapies that stimulate or block them, respectively.

MicroRNAs represent a relatively new class of gene expression regulators. Though already described in the early nineties ${ }^{4}$, their wide importance for maintaining homeostasis in virtually all human cell types has only been recognized in the past decade. They form a class of highly conserved small RNA molecules (20-23 nucleotides long) that do not encode for proteins, but that can influence the expression of protein-coding genes ${ }^{5}$. MicroRNAs bind to target messenger RNAs (mRNA) through complementarity between microRNA sequences and sequences in the mRNA 3' untranslated regions. This binding causes inhibition of target mRNA protein synthesis, either through mRNA destabilization or translational inhibition 5 . While microRNAs have a modest influence on the expression of individual target genes, they have profound effects on cellular biology through concerted inhibition of up to hundreds of genes ${ }^{6}$. MicroRNAs therefore act as post-transcriptional fine-tuners of gene expression. More than 1000 microRNAs have currently been identified in humans and their targetome is predicted to cover over $50 \%$ of all human protein-coding genes $^{7,8}$. MicroRNAs are indispensable for cardiac development and homeostasis ${ }^{9,10}$. Additionally, many cardiac diseases, including hypertrophic remodeling and heart failure, are associated with altered microRNA expression patterns ${ }^{11,12}$ which in turn may affect disease development and progression ${ }^{13}$. For instance, miR-199b expression is induced by the transcription factor NFAT during pressure overload-induced heart failure, and contributes to disease progression by further promoting NFAT activity in a positive feedback loop ${ }^{14}$. Similarly, many individual miRNAs have been linked to specific cardiac diseases including hypertrophic remodeling (miR-19914, miR-208a ${ }^{15}$ ), fibrosis (miR-2 $1^{16}$, miR-29 ${ }^{17}$ ), ischemia (miR-2418, miR-49919, miR-92a ${ }^{20}$ ), and arrhythmias (miR-121). 
This thesis focuses on the role of microRNAs in the regulation of cardiac inflammation, which represents an important common characteristic of different cardiac diseases ${ }^{22}$. Inflammatory responses involve coordinated activities by cells from the innate (such as macrophages and granulocytes) and adaptive immune system (predominantly $T$ and $B$ lymphocytes) in response to infection or tissue damage. The importance and relative contribution of such responses to either adaptive or maladaptive mechanisms varies widely per disease and depends on adequate dosing of the response. For instance, while inflammation after myocardial infarction is important for the clearance of dead cells and scar formation, excessive inflammation can cause wall rupture ${ }^{22}$. During pressured overload secreted factors from infiltrating inflammatory cells promote fibrosis through stimulating cardiac fibroblasts, or directly enhance the development of cardiomyocyte hypertrophy and arterial hypertension ${ }^{23-25}$. Elevated cytokine levels in heart failure cause myocyte death and suppressed contractility, thereby contributing to heart failure progression in a positive feedback loop ${ }^{26}$. In viral myocarditis, immune responses eliminate virus from the infected heart but simultaneously inflict irreversible damage to the myocardium ${ }^{27}$. Both the initiation and resolution of inflammatory responses are tightly regulated by various signaling mechanisms including the orchestration of inflammatory gene expression by microRNAs ${ }^{28}$. However, the involvement of microRNAs in cardiac inflammation and their contribution to cardiac pathologies has hitherto remained unstudied. Such studies are attractive since microRNAs are therapeutically targetable regulators of physiology and pathology while inflammation is an important focus of therapeutically driven cardiovascular research ${ }^{29}$.

In addition to their intracellular function as regulators of gene expression, microRNAs can also be detected in circulating plasma ${ }^{30}$. Though the mechanisms by which microRNAs exit cells and enter the blood stream are incompletely understood, plasma microRNAs are protected from degradation by exonucleases through interaction with (lipo)proteins or packaging in exosomes or microvesiscles, theoretically allowing their use as biomarkers ${ }^{31}$. Pioneer studies have shown the feasibility of cancer diagnosis through detection of tumorspecific microRNAs ${ }^{32}$, fostering interest in the use of plasma microRNAs as biomarkers for cardiovascular disease. At the time that research for this thesis commenced, no exploratory studies of plasma microRNAs as biomarkers in cardiac diseases were available".

\section{Aims and outline of this thesis}

The first goal of this thesis was to investigate the biological contribution of microRNAs to cardiac inflammation. To address this issue, two approaches were used. First, we profiled microRNA expression during virus-induced myocarditis, which by definition involves inflammation of the heart. This allowed us to identify microRNAs involved in cardiac inflammation in an unbiased manner. Next, we analyzed the functional importance of a known inflammatory microRNA, microRNA-155, during pressure overload-induced hypertrophy and heart failure. The second goal of this thesis was to explore the utility of 
circulating microRNAs for the diagnosis of cardiovascular disorders. Hereto, plasma levels of microRNAs were examined in patients with different cardiovascular diseases, including acute myocardial infarction, heart failure and myocarditis and comparing them to appropriate controls.

Chapters 2 and 3 of this thesis provide a more comprehensive background for the aforementioned studies. Chapter 2 outlines basic concepts of microRNA biology, including biogenesis, mode of action and plasma stability, while Chapter 3 discusses the pleiotropic role of inflammation in viral myocarditis. Chapters 4 through 7 represent the experimental studies performed. MicroRNA profiling and the functional roles of microRNA-155 and the microRNA-221-222 family in viral myocarditis are discussed in Chapters 4 and 5 . Chapter 6 describes the importance of microRNA-155 for cardiac hypertrophy and failure. The feasibility of using plasma microRNA as biomarkers for cardiovascular disease diagnosis in patients is described in Chapter 7. 


\section{References}

1. Dickstein K, Cohen-Solal A, Filippatos G, McMurray JJ, Ponikowski P, Poole-Wilson PA, Stromberg A, van Veldhuisen DJ, Atar D, Hoes AW, Keren A, Mebazaa A, Nieminen M, Priori SG, Swedberg K. Esc guidelines for the diagnosis and treatment of acute and chronic heart failure 2008: The task force for the diagnosis and treatment of acute and chronic heart failure 2008 of the european society of cardiology. Developed in collaboration with the heart failure association of the esc ( $\mathrm{hfa}$ ) and endorsed by the european society of intensive care medicine (esicm). European heart journal. 2008;29:2388-2442

2. Jessup M, Brozena S. Heart failure. The New England journal of medicine. 2003;348:2007-2018

3. Mudd JO, Kass DA. Tackling heart failure in the twenty-first century. Nature. 2008;451:919-928

4. Lee RC, Feinbaum RL, Ambros V. The c. Elegans heterochronic gene lin-4 encodes small rnas with antisense complementarity to lin-14. Cell. 1993;75:843-854

5. Bartel DP. Micrornas: Target recognition and regulatory functions. Cell. 2009;136:215-233

6. Baek D, Villen J, Shin C, Camargo FD, Gygi SP, Bartel DP. The impact of micrornas on protein output. Nature. 2008;455:64-71

7. Lewis BP, Burge CB, Bartel DP. Conserved seed pairing, often flanked by adenosines, indicates that thousands of human genes are microrna targets. Cell. 2005;120:15-20

8. Friedman RC, Farh KK, Burge CB, Bartel DP. Most mammalian mrnas are conserved targets of micrornas. Genome research. 2009;19:92-105

9. Chen JF, Murchison EP, Tang R, Callis TE, Tatsuguchi M, Deng Z, Rojas M, Hammond SM, Schneider MD, Selzman CH, Meissner G, Patterson C, Hannon GJ, Wang DZ. Targeted deletion of dicer in the heart leads to dilated cardiomyopathy and heart failure. Proceedings of the National Academy of Sciences of the United States of America. 2008;105:2111-2116

10. da Costa Martins PA, Bourajjaj M, Gladka M, Kortland M, van Oort RJ, Pinto YM, Molkentin JD, De Windt LJ. Conditional dicer gene deletion in the postnatal myocardium provokes spontaneous cardiac remodeling. Circulation. 2008;118:1567-1576

11. van Rooij E, Sutherland LB, Liu N, Williams AH, McAnally J, Gerard RD, Richardson JA, Olson EN. A signature pattern of stress-responsive micrornas that can evoke cardiac hypertrophy and heart failure. Proc Natl Acad Sci U S A. 2006;103:18255-18260

12. Thum T, Galuppo P, Wolf C, Fiedler J, Kneitz S, van Laake LW, Doevendans PA, Mummery CL, Borlak J, Haverich A, Gross C, Engelhardt S, Ertl G, Bauersachs J. Micrornas in the human heart: A clue to fetal gene reprogramming in heart failure. Circulation. 2007;116:258-267

13. Schroen B, Heymans S. Micrornas and beyond: The heart reveals its treasures. Hypertension. 2009;54:11891194

14. da Costa Martins PA, Salic K, Gladka MM, Armand AS, Leptidis S, el Azzouzi H, Hansen A, Coenen-de Roo CJ, Bierhuizen MF, van der Nagel R, van Kuik J, de Weger R, de Bruin A, Condorelli G, Arbones ML, Eschenhagen T, De Windt LJ. Microrna-199b targets the nuclear kinase dyrk1a in an auto-amplification loop promoting calcineurin/nfat signalling. Nature cell biology. 2010;12:1220-1227

15. van Rooij E, Sutherland LB, QiX, Richardson JA, Hill J, Olson EN. Control of stress-dependent cardiac growth and gene expression by a microrna. Science. 2007;316:575-579

16. Thum T, Gross C, Fiedler J, Fischer T, Kissler S, Bussen M, Galuppo P, Just S, Rottbauer W, Frantz S, Castoldi M, Soutschek J, Koteliansky V, Rosenwald A, Basson MA, Licht JD, Pena JT, Rouhanifard SH, Muckenthaler MU, Tuschl T, Martin GR, Bauersachs J, Engelhardt S. Microrna-21 contributes to myocardial disease by stimulating map kinase signalling in fibroblasts. Nature. 2008;456:980-984

17. van Rooij E, Sutherland LB, Thatcher JE, DiMaio JM, Naseem RH, Marshall WS, Hill JA, Olson EN. Dysregulation of micrornas after myocardial infarction reveals a role of mir-29 in cardiac fibrosis. Proc Natl Acad Sci U S A. 2008; 105:13027-13032 
18. Fiedler J, Jazbutyte V, Kirchmaier BC, Gupta SK, Lorenzen J, Hartmann D, Galuppo P, Kneitz S, Pena JT, SohnLee C, Loyer X, Soutschek J, Brand T, Tuschl T, Heineke J, Martin U, Schulte-Merker S, Ertl G, Engelhardt S, Bauersachs J, Thum T. Microrna-24 regulates vascularity after myocardial infarction. Circulation. 2011;124:720730

19. Wang JX, Jiao JQ, Li Q, Long B, Wang K, Liu JP, Li YR, Li PF. Mir-499 regulates mitochondrial dynamics by targeting calcineurin and dynamin-related protein-1. Nature medicine. 2011;17:71-78

20. Bonauer A, Carmona G, Iwasaki M, Mione M, Koyanagi M, Fischer A, Burchfield J, Fox H, Doebele C, Ohtani K, Chavakis E, Potente M, Tjwa M, Urbich C, Zeiher AM, Dimmeler S. Microrna-92a controls angiogenesis and functional recovery of ischemic tissues in mice. Science. 2009;324:1710-1713

21. Yang B, Lin H, Xiao J, Lu Y, Luo X, Li B, Zhang Y, Xu C, Bai Y, Wang H, Chen G, Wang Z. The muscle-specific microrna mir-1 regulates cardiac arrhythmogenic potential by targeting gja1 and kcnj2. Nat Med. 2007;13:486491

22. Marchant DJ, Boyd JH, Lin DC, Granville DJ, Garmaroudi FS, McManus BM. Inflammation in myocardial diseases. Circulation research. 2012;110:126-144

23. Kvakan H, Kleinewietfeld M, Qadri F, Park JK, Fischer R, Schwarz I, Rahn HP, Plehm R, Wellner M, Elitok S, Gratze P, Dechend R, Luft FC, Muller DN. Regulatory t cells ameliorate angiotensin ii-induced cardiac damage. Circulation. 2009;119:2904-2912

24. Guzik TJ, Hoch NE, Brown KA, McCann LA, Rahman A, Dikalov S, Goronzy J, Weyand C, Harrison DG. Role of the $t$ cell in the genesis of angiotensin ii induced hypertension and vascular dysfunction. The Journal of experimental medicine. 2007;204:2449-2460

25. Coles B, Fielding CA, Rose-John S, Scheller J, Jones SA, O'Donnell VB. Classic interleukin-6 receptor signaling and interleukin-6 trans-signaling differentially control angiotensin ii-dependent hypertension, cardiac signal transducer and activator of transcription-3 activation, and vascular hypertrophy in vivo. The American journal of pathology. 2007;171:315-325

26. Mann DL. Inflammatory mediators and the failing heart: Past, present, and the foreseeable future. Circulation research. 2002;91:988-998

27. Cooper LT, Jr. Myocarditis. N Engl J Med. 2009;360:1526-1538

28. O'Connell RM, Rao DS, Chaudhuri AA, Baltimore D. Physiological and pathological roles for micrornas in the immune system. Nature reviews. Immunology. 2010;10:111-122

29. Heymans S, Hirsch E, Anker SD, Aukrust P, Balligand JL, Cohen-Tervaert JW, Drexler H, Filippatos G, Felix SB, Gullestad L, Hilfiker-Kleiner D, Janssens S, Latini R, Neubauer G, Paulus WJ, Pieske B, Ponikowski P, Schroen B, Schultheiss HP, Tschope C, Van Bilsen M, Zannad F, McMurray J, Shah AM. Inflammation as a therapeutic target in heart failure? A scientific statement from the translational research committee of the heart failure association of the european society of cardiology. Eur J Heart Fail. 2009;11:119-129

30. Gilad S, Meiri E, Yogev Y, Benjamin S, Lebanony D, Yerushalmi N, Benjamin H, Kushnir M, Cholakh H, Melamed N, Bentwich Z, Hod M, Goren Y, Chajut A. Serum micrornas are promising novel biomarkers. PLoS ONE. 2008:3:e3148

31. Fichtlscherer S, Zeiher AM, Dimmeler S. Circulating micrornas: Biomarkers or mediators of cardiovascular diseases? Arteriosclerosis, thrombosis, and vascular biology. 2011;31:2383-2390

32. Mitchell PS, Parkin RK, Kroh EM, Fritz BR, Wyman SK, Pogosova-Agadjanyan EL, Peterson A, Noteboom J, O'Briant KC, Allen A, Lin DW, Urban N, Drescher CW, Knudsen BS, Stirewalt DL, Gentleman R, Vessella RL, Nelson PS, Martin DB, Tewari M. Circulating micrornas as stable blood-based markers for cancer detection. Proc Natl Acad Sci U S A. 2008;105:10513-10518 

MicroRNA Biology 


\section{Introduction}

MicroRNAs (miRNAs) are short RNAs of 20-23 nucleotides in length that are involved in the post-transcriptional regulation of gene expression in animals and plants ${ }^{1}$. The first miRNAs were discovered in a screen for genes that control developmental timing in worms: antisense complementarity between the small RNAs lin-4 and let-7 and messenger RNA (mRNA) of protein-coding heterochronic genes repressed their protein expression ${ }^{2,3}$. Lin-4 and let-7 appeared exemplary for a large class of small endogenous RNAs able to bind to partially complementary mRNAs and repress their protein levels. MiRNAs are expressed by in plants, algae, viruses, bacteria, fungi and mammals and are remarkably conserved throughout phylogeny, suggesting importance for competitive fitness ${ }^{4}$. The number of miRNAs identified in humans is currently approaching one thousand, many of which are expressed in a tissueand time-dependent manner. Single miRNAs can target up to hundreds of mRNAs. Consequently, human miRNAs are ramified to collectively regulate the expression of more than half of all protein-coding genes ${ }^{5,6}$. MiRNAs affect virtually every cellular process in eukaryote physiology including development, growth, energy metabolism, and cell death. In addition, a wide range of diseases is associated with differential miRNA expression, including cancer and cardiovascular diseases ${ }^{7,8}$. This chapter aims to summarize elementary aspects of miRNA biogenesis and their function in cardiovascular physiology and diseases. Finally, the recent finding that miRNAs are detectable in circulating plasma and may be used as biomarkers for cardiovascular disease will be discussed.

\section{MicroRNA biogenesis}

Mature miRNAs are processed from precursor molecules (primary or pri-miRNAs), which are transcribed from independent miRNA genes or processed from the introns of proteincoding genes ${ }^{9}$. Transcription of pri-miRNA is mediated by the transcription factor-dependent polymerase II, allowing dynamic regulation of pri-miRNA levels (Figure 1). Typically, primiRNAs are long transcripts that contain one or more local hairpins harboring a mature miRNA. Canonical processing of pri-miRNAs involves two steps of enzymatic processing by the RNAse III family members Drosha and Dicer, respectively ${ }^{5}$. In the first step, Drosha cleaves the pri-miRNA in the nucleus to yield a 70 nt hairpin precursor (pre-miRNA). Alternatively, non-canonical processing involves the Drosha-independent generation of premiRNA directly through splicing of short introns (called mirtrons). Both mechanisms converge when the pre-miRNA precursor is shuttled from the nucleus to the cytoplasm by exportin- 5 . This process is energy-dependent and follows exportin- 5 recognition of the 3 ' overhang of the pre-miRNA caused by Drosha cleavage ${ }^{10}$. Once in the cytoplasm, the stem-loop structure of the pre-miRNA hairpin is cleaved by Dicer, thereby removing the loop to yield a small 20 to 23-bp double stranded miRNA/miRNA* duplex. Of this duplex, the strand with the least stable $5^{\prime}$ binding (the mature miRNA) will be incorporated into the microRNA-induced silencing complex (miRISC), where it acts as guide strand to complementary messages. The other strand, referred to as the passenger or miRNA* strand, is often degraded ${ }^{11}$. 


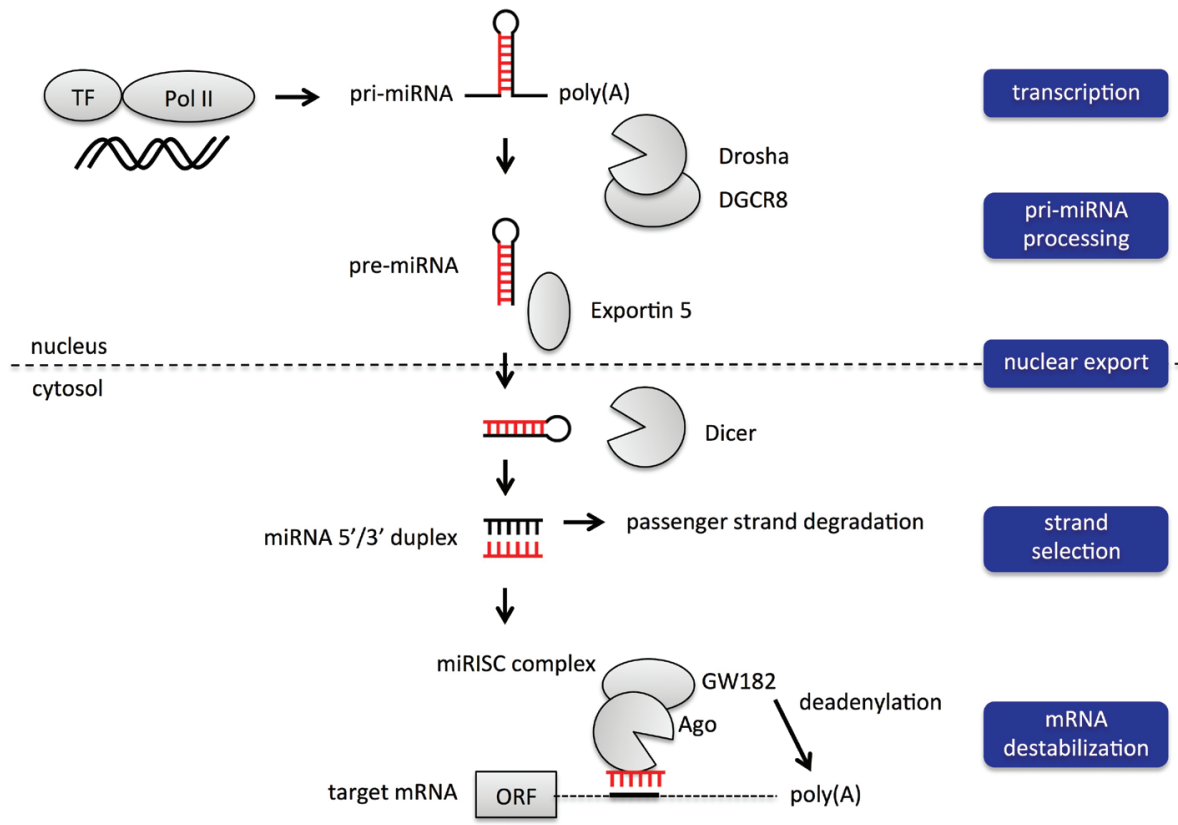

Figure 1. MicroRNA biogenesis. MicroRNAs (miRNAs) are transcribed from independent genes or from non-coding parts of other genes by polymerase II (Pol II). Their rate of transcription is dynamically regulated by transcription factors (TF), similar to protein-coding genes, and yields poly-adenylated primary precursor transcripts (pri-miRNA) that require two steps of enzymatic processing for the generation of mature miRNAs. In the nucleus, pri-miRNAs are cleaved by the RNAse III enzyme Drosha into hairpin precursors (pre-miRNAs), before being actively exported from the nucleus by Exportin 5. The process of primiRNA cleavage by Drosha is regulated by a number of accessory proteins, such as DGCR8. In the cytosol, Dicer cleaves the stem-loop structure from the miRNA/miRNA* duplex, thereby yielding a 20 to 23-bp double stranded miRNA/miRNA* duplex. Incorporation of the miRNA or miRNA* strand into RNA-induced silencing complexes (RISC) depends on thermodynamic strand stability and varies between miRNAs. Once incorporated, miRNAs guide the silencing effect of the RISC complex by binding to complementary sequences in the 3' untranslated regions (3'UTR) downstream of the open reading frame (ORF) of target messenger RNAs (mRNA). Target mRNA degradation of translational repression is effectuated by effector proteins in RISC, such as Argonaute (Ago) and GW182.

Of note, miRNA* strands are not always waste products of miRNA biogenesis and can also be loaded into miRISC complexes. Ratios of miRNA/miRNA* loading into miRISC are in fact variable between cell types and states ${ }^{5}$. This insight has led to a recent strand reclassification in miRBase, the most widely used online miRNA database, which since 2012 differentiates between a miRNA-3p/miRNA-5p strand rather than miRNA/miRNA*.

\section{MicroRNA function}

MiRNAs are involved in a mechanism called transcriptional silencing. By binding to complementary regions in the 3'UTR of target mRNAs, miRNAs guide the actions of effector proteins in the miRISC complex, including Argonaute (Ago) and glycine-tryptophan protein of 
$182 \mathrm{kDa}(\mathrm{GW} 182)^{5}$. These effector proteins generally inhibit the protein output from that message through a variety of mechanisms, including degradation, destabilization or translational repression of the message ${ }^{12}$. Interestingly, the modes of miRNA function in plants and animals are different. In plants, miRNAs bind to targets with extensive complementary for the mature miRNA sequence which induces direct cleavage of the targeted message. Though this mechanism is also present in animals, it is uncommon and such extensive complementarity is rare ${ }^{1}$. In contrast, metazoan miRNA binding to target sequences strongly relies on $3^{\prime} U T R$ complementarity to the first 8 nucleotides of the miRNA's $5^{\prime}$ end, called the seed region. It is currently not possible to deduce definite targets for miRNAs based on sequence information available in public databases, and experimental validation of targets using luciferase reporters tailed by the 3'UTR of interest remains the golden standard. However, improvements in target prediction algorithms have significantly reduced the false positive rates of such predictions over the past decade ${ }^{1}$. One important reason for this improvement has been the incorporation of evolutionary conservation into the prediction methods ${ }^{13}$. Targets sites that are conserved more than would be expected by chance are considered to be under selective pressure and likely biologically relevant. Evolutionary conservation of target sites therefore adds confidence to the prediction as compared to site with similar complementarity for which mutations have gone unpunished ${ }^{13}$. However, predicted non-conserved binding sites for miRNAs outnumber conserved binding sites about ten-to-one', and many non-conserved predicted targets are increased in abundance following miRNA deletion in high-throughput analyses ${ }^{14,15}$. Since 3'UTR regions of genes harbor a high frequency of single nucleotide polymorphisms in comparison to protein coding regions, evolving generation and disruption of miRNA binding sites may reflect an evolutionary strategy that promotes quick and nuanced adaptations in gene expression. In conclusion, while some of such non-conserved targeting may represent biologically inconsequential interactions, species-specific miRNA regulation may contribute to biological homeostasis or pathology in humans.

\section{direct targets indirect targets}

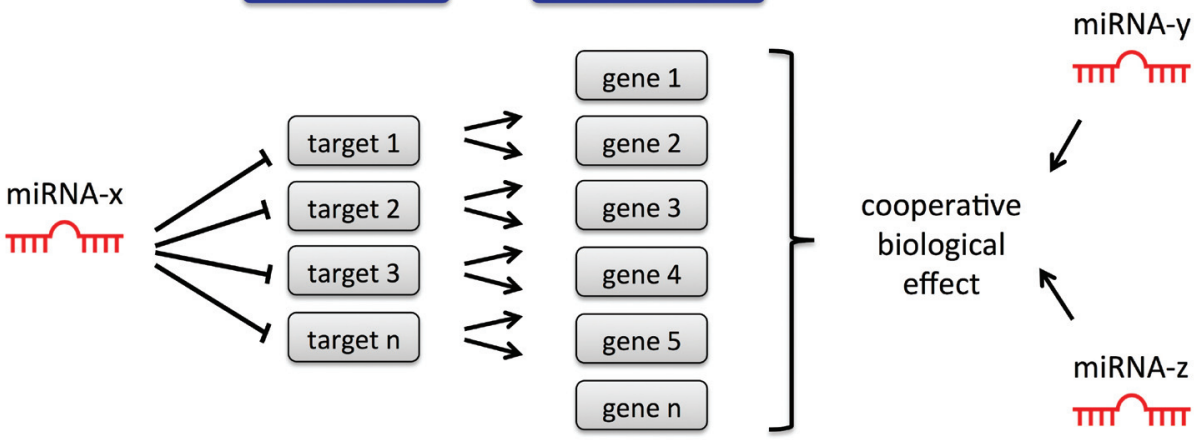

Figure 2. MiRNAs influence biology through gene networks. MiRNAs can directly target hundreds of targets (miR-x in the figure). In turn, these targets can affect the transcription, stability or localization of a large number or indirect target messenger RNA or proteins. Phenotypic effects of altered miRNA signaling are therefore the resultants of small changes in entire signaling networks. In turn, such phenotypical traits are ofter regulated by multiple miRNAs at the same time (miR-y and miR-z). 
Multiple mechanisms for miRNA target silencing exist, raising the question which of them is dominant in mammalian biology. While initial studies suggested that miRNAs reduced target protein output through translational repression without affecting mRNA levels ${ }^{12,16}$, later mRNA-array studies demonstrated that many miRNA target mRNA levels are decreased in response to miRNA overexpression, which was associated with shortening of the poly $(A)$ tail ${ }^{17-19}$. This mechanism is known as mRNA destabilization and is mediated by the effector protein GW182 in the miRISC complex, which can promote transcript deadenylation followed by mRNA decapping and destabilization ${ }^{20}$. Since the effect of miRNAs on transcript levels is generally small, a prevailing belief has been that translational repression is the dominant mode of miRNA action in mammals. This belief has been challenged by recent evidence that miRNA-associated reductions in ribosome-associated target mRNA (nascent proteins) are paralleled by whole-cell mRNA reductions and that $84 \%$ of target repression effects are mediated by mRNA destabilization rather than translational repression ${ }^{12}$. Observations of stronger miRNA effects on the protein than mRNA level therefore likely reflect the methodological sampling difference between transcriptomics and proteomics: small differences in snapshot measurements of mRNAs with short half-lives may be amplified in stably accumulated protein levels. Though our knowledge about miRNA targeting has significantly increased in the past years, much remains to be learned about the different silencing mechanisms and the factors determining which mechanism will be activated following miRNA binding.

\section{MiRNA impact on gene networks}

A consistent observation of miRNA target regulation is that miRNAs suppress their individual targets only to a mild degree. High throughput proteomics analyses have revealed that protein levels of most miRNA targets with perfect seed matching change only $10-20 \%$ after miRNA overexpression or knockout ${ }^{14,15}$. Conversely, the same studies showed that hundreds of direct miRNA targets are affected by such miRNA manipulations, and that direct and indirect effects cooperatively regulate the expression of up to thousands of genes (Figure 2). MiRNAs therefore direct their effects through a collection of nuanced perturbations, which may be a favorable aspect for their use as therapeutic targets ${ }^{21}$. The flipside of this regulatory mechanism is that it complicates the identification or miRNA targets that explain observed phenotypes, as focusing on single targets may not be sufficient to explain the cooperative effects of targets in reality.

It is tempting to speculate that miRNA targets should functionally relate to cooperatively orchestrate biological functions. Target sets of some microRNAs are indeed enriched for specific functions. For example, the miR-17 92 cluster has well-known oncogenic properties $^{22}$ and is enriched in target genes with a role in growth control ${ }^{23}$. Such enrichment is nonetheless not always observed and many miRNAs seem to influence a range of different processes. Moreover, their functions can vary across different cell types based on the differential availability of miRNA targets in such cell types1. Vice versa, many biological functions are regulated by different miRNAs simultaneously. In line with such 
observations, many individual miRNA knockouts in worms lack overt phenotypes due to buffering by both miRNA family members and non-family members ${ }^{24}$, 25 The same phenomenon occurs in mice in cardiovascular research, where single miRNA knockouts often fail to produce deviant phenotypes until challenged by disease models, such as myocardial infarction or aortic banding ${ }^{7}$.

\section{Regulation of miRNA activity}

As regulators of gene expression, miRNAs themselves are also subject to sophisticated control. Promoters of miRNA genes are very similar to those of coding genes and contain elements that suggest cis-regulation of transcription, including TATA box sequences, CpG islands, initiation elements and histone modifications ${ }^{5}$. Following transcription, processing of pri-miRNAs by Drosha and Dicer is influenced by the abundance and activity of accessory proteins such as DGCR8, which stabilizes the Drosha microprocessor complex to increase pri-miRNA processing ${ }^{26}$. Some accessory proteins selectively influence processing of specific miRNAs or families, such as Lin-28 that binds to the terminal loop of pre-let-7 and interferes with Drosha cleavage ${ }^{27}$. After processing and incorporation into the miRISC complex, miRNA-interactions are affected by levels of the miRISC core proteins Argonaute and GW182, and by dozens of additional proteins that either counteract or facilitate miRNA target repression ${ }^{5}$. Such repression is also influenced by the intracellular localization of miRNAs: many cells contain cytoplasmic foci known as processing bodies (p-bodies) that are enriched in miRNA, mRNA, Dicer and Argonaute. Disruption of miRNA biogenesis leads to disappearance of p-bodies suggesting that they play a functional role in miRNA biology 28 . The turnover of miRNAs may also affect miRNA levels and function, but has not extensively been studied thus far.

MiRNAs are also subject to accelerated decay under certain cellular conditions. For instance, miR-29b decays quicker in actively proliferating cells as compared to cells arrested in mitosis ${ }^{29}$. Though the underlying mechanisms are incompletely understood, it is likely that miRNA turnover is an important determinant of miRNA function. Finally, highly proliferating or activated cells tend to produce mRNAs with shorter 3'UTRs that are less sensitive to miRNA-mediated repression ${ }^{30}$. In conclusion, endogenous regulation of miRNA function appears as complex as miRNA targetome silencing itself and adds to the complexity of miRNA biology.

\section{Roles of miRNAs in the heart}

The heart expresses approximately 200 different miRNAs including abundant levels of the cardiomyocytes-specific miR-208a, muscle-specific miRNAs including miR-1, miR-133, miR499, miR-206, and miR-208b, as well as non-myocyte specific miRNAs such as miR-29a, miR-26a, and members of the let-7 family ${ }^{31,32}$. Interruption of miRNA biogenesis in cardiomyocytes through targeted myocyte-specific deletion of Dicer in mice results in 
Endothelial cell

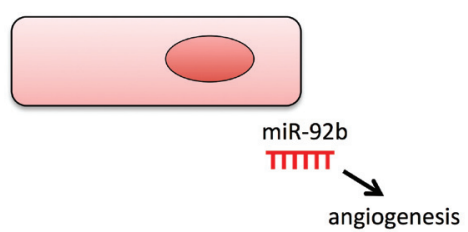

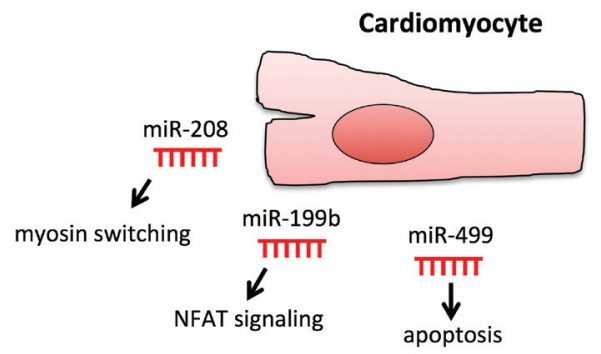

\section{heart failure}
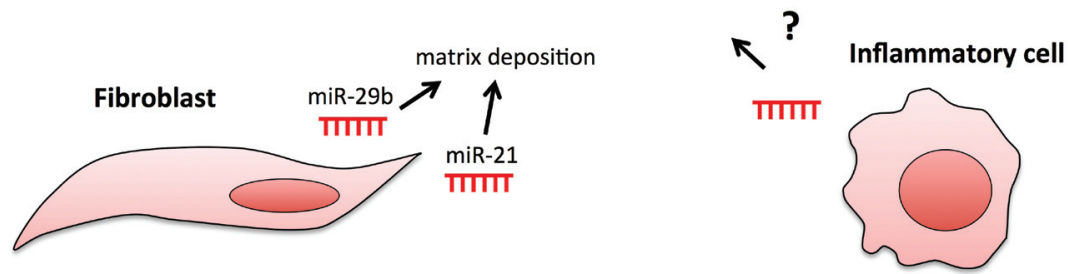

Figure 3. MiRNA roles in different cardiac cell types during cardiac disorders. The onset and progression of heart failure is impacted by miRNA signaling in different cardiac cell types. For instance, miR-199b and miR-208 are highly expressed in cardiomyocytes and affect NFAT signaling and myosin switching, respectively; two signaling cascades with important roles in heart failure. Similarly, miR-499 affect cardiomyocyte apoptosis. In addition to cardiomyocytes, miRNAs in cardiac fibroblasts (miR-21, miR29b) and endothelial cells (miR-92b) also influence cardiac failure. In contrast, while immune activation is important in many forms of heart failure, contributions of individual miRNAs expressed by inflammatory cells on cardiac disease have not been reported so far.

embryonic mortality or rapidly progressing postnatal heart failure and death ${ }^{33,34}$. In addition, targeted dicer deletion in cardiomyocytes of adult mice leads to severe dilated cardiomyopathy, indicating that miRNA regulation of cardiomyocytes homeostasis remains essential beyond the developmental phase ${ }^{35}$. In subsequent studies, multiple individual miRNAs have been identified to play important roles in cardiac development and diseases $7,36,37$. During normal heart development, embryonic stem cell commitment to the mesodermal lineage is cooperatively stimulated by miR-1 and miR-133, two highly expressed myocyte-specific miRNAs that are transcribed from a common pri-miRNA precursor. Subsequently, miR-1 promotes further differentiation into cardiomyocytes while miR-133 inhibits this transition ${ }^{38}$. These actions of miR-1 and miR-133 are buffered to some degree by each other and other miRNAs, since half of the miR-1 or miR-133 knockout mice are viable ${ }^{34,39}$. Several miRNAs that are highly expressed in cardiomyocytes impact the onset and progression of cardiac diseases such as hypertrophic remodeling or dilated cardiomyopathy ${ }^{40-42}$. In addition, miRNAs in other cardiac cells, such as fibroblasts and endothelial cells, also affect cardiac pathophysiology (Figure 3). To give a few examples, miR-21 is up-regulated in fibroblasts during pressure overload and contributes to cardiac hypertrophy and fibrosis ${ }^{43}$. After myocardial infarction, fibroblasts in the ischemic border zone down-regulate miR-29b to derepress pro-fibrotic genes leading to collagen deposition. In endothelial cells, miR-92a suppresses pro-angiogenic factors and miR-92a inhibition in vivo in mice results in improved cardiac angiogenesis and function after myocardial infarction ${ }^{44}$. An important cardiac cell 
type that has received little attention thus far is the leukocyte: the heart harbors a population of resident macrophages or dendritic cells under basal conditions and many cardiac diseases are characterized by pronounced activation of inflammatory signaling associated with the influx of recruited inflammatory cells into the heart ${ }^{45}$. Since inflammation is subject to stringent miRNA control ${ }^{46}$, the impact of inflammatory miRNA signaling in cardiac disease represents a gap in our current knowledge that mandates investigation.

\section{Circulating microRNAs}

Beyond their intracellular function, miRNAs have recently been found in several bodily fluids, including blood and cell-free fractions such as plasma, serum and urine ${ }^{47}$. MiRNA levels in such fluids are remarkably stable and resistant to a broad range of damaging conditions, including boiling, repeated freeze-thaw cycles, prolonged storage at room temperature, and acidic or basic $\mathrm{pH}$ environments ${ }^{48-50}$. In contrast, when short RNAs are exogenously added to plasma they are rapidly degraded by RNAse, suggesting that endogenous plasma miRNAs are shielded from exonucleases activity ${ }^{48}$. Indeed, miRNAs are stably transported through the circulation by incorporation into vesicles such as endosome-derived exosomes and cell membrane-derived microvesicles or apoptotic bodies or through association with lipoproteins like high-density lipoprotein (HDL) $)^{51-54}$. In addition, a large fraction of plasma miRNAs is protected by directly associating with chaperone proteins such as Argonaute or by Nucleophosmin-1 (NMP1)55, 56. This protein-bound fraction is estimated to represent approximately $90 \%$ of circulating miRNAs in human plasma ${ }^{55,57}$. Individual miRNAs can be preferentially exported into either vesicle- or protein-associated miRNA ${ }^{55}$, and their altered release under stressed or transformed conditions appears a selective process ${ }^{58}$. However, much remains to be learned about the factors that determine cellular miRNA export and their physiological significance. The stable detectability of miRNAs in patient samples nonetheless offers direct diagnostic opportunities. MiRNAs are detected in plasma with high sensitivity and specificity, and plasma measurement of tumor-specific miRNAs allows accurate separation of healthy subjects from patients with a variety of malignancies ${ }^{48-50}$ and identification of diabetic or pre-diabetic patients ${ }^{49,59}$. Therefore, the use of single or combined plasma miRNA biomarkers may also aid in the diagnosis or prognosis of a range of cardiovascular diseases, such as acute coronary syndrome, heart failure and viral myocarditis.

\section{Conclusions}

The broad importance of miRNAs for cardiovascular development, physiology, and disease are by now well established. A major challenge lies in the capitalization of such basic insights by translating them into diagnostic and therapeutic tools with clinical perspectives. Therapeutically, the multitude of subtle gene changes caused by individual miRNAs is attractive since this may allow for more 'physiologic' manipulation than single protein therapeutics. On the other hand, the prediction of potential side effects is complicated by 
miRNA versatility and will mandate careful analysis in clinical studies. Despite such concerns, selective miRNA inhibitors have been successfully used in non-human primates and are currently in phase II clinical trials ${ }^{60,61}$, providing basic researchers with an important stimulus to pursue screening for therapeutic miRNA candidates. In addition, the potential of miRNA use as biomarkers for cardiovascular disease invites experimental and clinical investigation and may be directly translatable to clinical decision-making. 


\section{References}

1. Bartel DP. Micrornas: Target recognition and regulatory functions. Cell. 2009;136:215-233

2. Lee RC, Feinbaum RL, Ambros V. The $c$. Elegans heterochronic gene lin-4 encodes small rnas with antisense complementarity to lin-14. Cell. 1993;75:843-854

3. Reinhart BJ, Slack FJ, Basson M, Pasquinelli AE, Bettinger JC, Rougvie AE, Horvitz HR, Ruvkun G. The 21-nucleotide let-7 rna regulates developmental timing in caenorhabditis elegans. Nature. 2000;403:901-906

4. Griffiths-Jones S, Saini HK, van Dongen S, Enright AJ. Mirbase: Tools for microrna genomics. Nucleic acids research. 2008;36:D154-158

5. Krol J, Loedige I, Filipowicz W. The widespread regulation of microrna biogenesis, function and decay. Nature reviews. Genetics. 2010;11:597-610

6. Friedman RC, Farh $\mathrm{KK}$, Burge $\mathrm{CB}$, Bartel DP. Most mammalian mrnas are conserved targets of micrornas. Genome research. 2009;19:92-105

7. Small EM, Olson EN. Pervasive roles of micrornas in cardiovascular biology. Nature. 2011;469:336-342

8. Esquela-Kerscher A, Slack FJ. Oncomirs - micrornas with a role in cancer. Nat Rev Cancer. 2006;6:259-269

9. Kim VN, Han J, Siomi MC. Biogenesis of small rnas in animals. Nature reviews. Molecular cell biology. 2009;10:126-139

10. Murchison EP, Hannon GJ. Mirnas on the move: Mirna biogenesis and the rnai machinery. Current opinion in cell biology. 2004;16:223-229

11. Okamura K, Liu N, Lai EC. Distinct mechanisms for microrna strand selection by drosophila argonautes. Molecular cell. 2009;36:431-444

12. Guo H, Ingolia NT, Weissman JS, Bartel DP. Mammalian micrornas predominantly act to decrease target mrna levels. Nature. 2010;466:835-840

13. Lewis BP, Shih IH, Jones-Rhoades MW, Bartel DP, Burge CB. Prediction of mammalian microrna targets. Cell. 2003;115:787-798

14. Baek D, Villen J, Shin C, Camargo FD, Gygi SP, Bartel DP. The impact of micrornas on protein output. Nature. 2008;455:64-71

15. Selbach M, Schwanhausser B, Thierfelder N, Fang Z, Khanin R, Rajewsky N. Widespread changes in protein synthesis induced by micrornas. Nature. 2008;455:58-63

16. Wightman B, Ha I, Ruvkun G. Posttranscriptional regulation of the heterochronic gene lin-14 by lin-4 mediates temporal pattern formation in c. Elegans. Cell. 1993;75:855-862

17. Bagga S, Bracht J, Hunter S, Massirer K, Holtz J, Eachus R, Pasquinelli AE. Regulation by let-7 and lin-4 mirnas results in target mrna degradation. Cell. 2005;122:553-563

18. Wu L, Fan J, Belasco JG. Micrornas direct rapid deadenylation of mrna. Proceedings of the National Academy of Sciences of the United States of America. 2006;103:4034-4039

19. Behm-Ansmant I, Rehwinkel J, Doerks T, Stark A, Bork P, Izaurralde E. Mrna degradation by mirnas and gw182 requires both ccr4:Not deadenylase and dcp1:Dcp2 decapping complexes. Genes \& development. 2006:20:1885-1898

20. Fabian MR, Sonenberg N, Filipowicz W. Regulation of mrna translation and stability by micrornas. Annual review of biochemistry. 2010;79:351-379

21. Wurdinger T, Costa FF. Molecular therapy in the microrna era. Pharmacogenomics J. 2007;7:297-304

22. He L, Thomson JM, Hemann MT, Hernando-Monge E, Mu D, Goodson S, Powers S, Cordon-Cardo C, Lowe SW, Hannon GJ, Hammond SM. A microrna polycistron as a potential human oncogene. Nature. 2005;435:828-833

23. Lewis BP, Burge CB, Bartel DP. Conserved seed pairing, often flanked by adenosines, indicates that thousands of human genes are microrna targets. Cell. 2005;120:15-20 
24. Brenner JL, Jasiewicz KL, Fahley AF, Kemp BJ, Abbott AL. Loss of individual micrornas causes mutant phenotypes in sensitized genetic backgrounds in c. Elegans. Current biology : CB. 2010;20:1321-1325

25. Alvarez-Saavedra E, Horvitz HR. Many families of c. Elegans micrornas are not essential for development or viability. Current biology : CB. 2010;20:367-373

26. Gregory RI, Yan KP, Amuthan G, Chendrimada T, Doratotaj B, Cooch N, Shiekhattar R. The microprocessor complex mediates the genesis of micrornas. Nature. 2004;432:235-240

27. Viswanathan SR, Daley GQ. Lin28: A microrna regulator with a macro role. Cell. 2010;140:445-449

28. Eulalio A, Behm-Ansmant I, Izaurralde E. P bodies: At the crossroads of post-transcriptional pathways. Nature reviews. Molecular cell biology. 2007;8:9-22

29. Hwang HW, Wentzel EA, Mendell JT. A hexanucleotide element directs microrna nuclear import. Science. 2007;315:97-100

30. Sandberg R, Neilson JR, Sarma A, Sharp PA, Burge CB. Proliferating cells express mrnas with shortened 3' untranslated regions and fewer microrna target sites. Science. 2008;320:1643-1647

31. Rao PK, Toyama Y, Chiang HR, Gupta S, Bauer M, Medvid R, Reinhardt F, Liao R, Krieger M, Jaenisch R, Lodish HF, Blelloch R. Loss of cardiac microrna-mediated regulation leads to dilated cardiomyopathy and heart failure. Circulation research. 2009;105:585-594

32. van Rooij E. Introduction to the series on micrornas in the cardiovascular system. Circulation research. $2012 ; 110: 481-482$

33. Chen JF, Murchison EP, Tang R, Callis TE, Tatsuguchi M, Deng Z, Rojas M, Hammond SM, Schneider MD, Selzman CH, Meissner G, Patterson C, Hannon GJ, Wang DZ. Targeted deletion of dicer in the heart leads to dilated cardiomyopathy and heart failure. Proceedings of the National Academy of Sciences of the United States of America. 2008;105:2111-2116

34. Zhao Y, Ransom JF, Li A, Vedantham V, von Drehle M, Muth AN, Tsuchihashi T, McManus MT, Schwartz RJ, Srivastava D. Dysregulation of cardiogenesis, cardiac conduction, and cell cycle in mice lacking mirna-1-2. Cell. 2007;129:303-317

35. da Costa Martins PA, Bourajjaj M, Gladka M, Kortland M, van Oort RJ, Pinto YM, Molkentin JD, De Windt LJ. Conditional dicer gene deletion in the postnatal myocardium provokes spontaneous cardiac remodeling. Circulation. 2008;118:1567-1576

36. Schroen B, Heymans S. Micrornas and beyond: The heart reveals its treasures. Hypertension. 2009;54:11891194

37. Condorelli G, Latronico MV, Dorn GW, 2nd. Micrornas in heart disease: Putative novel therapeutic targets? European heart journal. 2010;31:649-658

38. Ivey KN, Muth A, Arnold J, King FW, Yeh RF, Fish JE, Hsiao EC, Schwartz RJ, Conklin BR, Bernstein HS, Srivastava D. Microrna regulation of cell lineages in mouse and human embryonic stem cells. Cell stem cell. 2008;2:219-229

39. Liu N, Bezprozvannaya S, Williams AH, Oi X, Richardson JA, Bassel-Duby R, Olson EN. Microrna-133a regulates cardiomyocyte proliferation and suppresses smooth muscle gene expression in the heart. Genes Dev. 2008;22:3242-3254

40. van Rooij E, Sutherland LB, Liu N, Williams AH, McAnally J, Gerard RD, Richardson JA, Olson EN. A signature pattern of stress-responsive micrornas that can evoke cardiac hypertrophy and heart failure. Proc Natl Acad Sci U S A. 2006;103:18255-18260

41. van Rooij E, Sutherland LB, Qi X, Richardson JA, Hill J, Olson EN. Control of stress-dependent cardiac growth and gene expression by a microrna. Science. 2007;316:575-579

42. da Costa Martins PA, Salic K, Gladka MM, Armand AS, Leptidis S, el Azzouzi H, Hansen A, Coenen-de Roo CJ, Bierhuizen MF, van der Nagel R, van Kuik J, de Weger R, de Bruin A, Condorelli G, Arbones ML, Eschenhagen T, De Windt LJ. Microrna-199b targets the nuclear kinase dyrk1a in an auto-amplification loop promoting calcineurin/nfat signalling. Nature cell biology. 2010;12:1220-1227

43. Thum T, Gross C, Fiedler J, Fischer T, Kissler S, Bussen M, Galuppo P, Just S, Rottbauer W, Frantz S, Castoldi M, Soutschek J, Koteliansky V, Rosenwald A, Basson MA, Licht JD, Pena JT, Rouhanifard SH, Muckenthaler 
MU, Tuschl T, Martin GR, Bauersachs J, Engelhardt S. Microrna-21 contributes to myocardial disease by stimulating map kinase signalling in fibroblasts. Nature. 2008;456:980-984

44. Bonauer A, Carmona G, Iwasaki M, Mione M, Koyanagi M, Fischer A, Burchfield J, Fox H, Doebele C, Ohtani K, Chavakis E, Potente M, Tjwa M, Urbich C, Zeiher AM, Dimmeler S. Microrna-92a controls angiogenesis and functional recovery of ischemic tissues in mice. Science. 2009;324:1710-1713

45. Marchant DJ, Boyd JH, Lin DC, Granville DJ, Garmaroudi FS, McManus BM. Inflammation in myocardial diseases. Circulation research. 2012;110:126-144

46. O'Connell RM, Rao DS, Chaudhuri AA, Baltimore D. Physiological and pathological roles for micrornas in the immune system. Nature reviews. Immunology. 2010;10:111-122

47. Creemers EE, Tijsen AJ, Pinto YM. Circulating micrornas: Novel biomarkers and extracellular communicators in cardiovascular disease? Circulation research. 2012;110:483-495

48. Mitchell PS, Parkin RK, Kroh EM, Fritz BR, Wyman SK, Pogosova-Agadjanyan EL, Peterson A, Noteboom J, O'Briant KC, Allen A, Lin DW, Urban N, Drescher CW, Knudsen BS, Stirewalt DL, Gentleman R, Vessella RL, Nelson PS, Martin DB, Tewari M. Circulating micrornas as stable blood-based markers for cancer detection. Proc Natl Acad Sci U S A. 2008;105:10513-10518

49. Chen X, Ba Y, Ma L, Cai X, Yin Y, Wang K, Guo J, Zhang Y, Chen J, Guo X, Li Q, Li X, Wang W, Wang J, Jiang $X$, Xiang Y, Xu C, Zheng P, Zhang J, Li R, Zhang H, Shang X, Gong T, Ning G, Zen K, Zhang CY. Characterization of micrornas in serum: A novel class of biomarkers for diagnosis of cancer and other diseases. Cell Res. 2008;18:997-1006

50. Lawrie CH, Gal S, Dunlop HM, Pushkaran B, Liggins AP, Pulford K, Banham AH, Pezzella F, Boultwood J, Wainscoat JS, Hatton CS, Harris AL. Detection of elevated levels of tumour-associated micrornas in serum of patients with diffuse large b-cell lymphoma. Br J Haematol. 2008;141:672-675

51. Zernecke A, Bidzhekov K, Noels H, Shagdarsuren E, Gan L, Denecke B, Hristov M, Koppel T, Jahantigh MN, Lutgens E, Wang S, Olson EN, Schober A, Weber C. Delivery of microrna-126 by apoptotic bodies induces cxcl12-dependent vascular protection. Science signaling. 2009;2:ra81

52. Valadi H, Ekstrom K, Bossios A, Sjostrand M, Lee JJ, Lotvall JO. Exosome-mediated transfer of mrnas and micrornas is a novel mechanism of genetic exchange between cells. Nature cell biology. 2007;9:654-659

53. Vickers KC, Palmisano BT, Shoucri BM, Shamburek RD, Remaley AT. Micrornas are transported in plasma and delivered to recipient cells by high-density lipoproteins. Nature cell biology. 2011;13:423-433

54. Skog J, Wurdinger T, van Rijn S, Meijer DH, Gainche L, Sena-Esteves M, Curry WT, Jr., Carter BS, Krichevsky AM, Breakefield XO. Glioblastoma microvesicles transport rna and proteins that promote tumour growth and provide diagnostic biomarkers. Nat Cell Biol. 2008;10:1470-1476

55. Arroyo JD, Chevillet JR, Kroh EM, Ruf IK, Pritchard CC, Gibson DF, Mitchell PS, Bennett CF, PogosovaAgadjanyan EL, Stirewalt DL, Tait JF, Tewari M. Argonaute2 complexes carry a population of circulating micrornas independent of vesicles in human plasma. Proceedings of the National Academy of Sciences of the United States of America. 2011;108:5003-5008

56. Wang K, Zhang S, Weber J, Baxter D, Galas DJ. Export of micrornas and microrna-protective protein by mammalian cells. Nucleic acids research. 2010;38:7248-7259

57. Turchinovich A, Weiz L, Langheinz A, Burwinkel B. Characterization of extracellular circulating microrna. Nucleic acids research. 2011;39:7223-7233

58. Pigati L, Yaddanapudi SC, lyengar R, Kim DJ, Hearn SA, Danforth D, Hastings ML, Duelli DM. Selective release of microrna species from normal and malignant mammary epithelial cells. PLoS ONE. 2010;5:e13515

59. Zampetaki A, Kiechl S, Drozdov I, Willeit P, Mayr U, Prokopi M, Mayr A, Weger S, Oberhollenzer F, Bonora E, Shah A, Willeit J, Mayr M. Plasma microrna profiling reveals loss of endothelial mir-126 and other micrornas in type 2 diabetes. Circulation research. 2010;107:810-817

60. Elmen J, Lindow M, Schutz S, Lawrence M, Petri A, Obad S, Lindholm M, Hedtjarn M, Hansen HF, Berger U, Gullans S, Kearney P, Sarnow P, Straarup EM, Kauppinen S. Lna-mediated microrna silencing in non-human primates. Nature. 2008;452:896-899

61. Lanford RE, Hildebrandt-Eriksen ES, Petri A, Persson R, Lindow M, Munk ME, Kauppinen S, Orum H. Therapeutic silencing of microrna-122 in primates with chronic hepatitis c virus infection. Science. 2010;327:198-201 


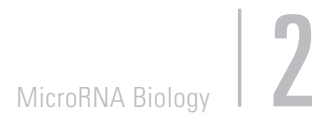


Maarten F. Corsten ${ }^{1}$

Blanche Schroen'

Stephane Heymans 1,2,3 $^{1}$

1 Center for Heart Failure Research, Cardiovascular Research Institute Maastricht (CARIM), Maastricht University, The Netherlands

2 Interuniversity Cardiology Institute of the Netherlands, Utrecht, The Netherlands

3 Cardiovascular Sciences Department, University of Leuven, Belgium 


\section{Inflammation in Viral Myocarditis; Friend or Foe?}

Modified from Trends in Molecular Medicine 2012;18:426-437 [IF 10.3]

\section{ABSTRACT}

Viral myocarditis is an important cause of heart failure for which no specific treatments are available. Direct viral injury to cardiac cells provokes an inflammatory response that significantly contributes to cardiac damage and ensuing morbidity. Despite the central pathogenic role of autoimmune injury, broad inhibition of the inflammatory response does not result in patient benefit. Many preclinical studies collectively emphasize that modulating distinct inflammatory signaling pathways may yield effective viral clearance while preserving cardiac structure. This review aims to provide an overview of the sometimes-contrasting observations from experimental viral myocarditis models and to translate the lessons learned into opportunities for future investigations and therapies. 


\section{Background}

Myocarditis is broadly defined as an inflammatory condition of the heart. It represents one of the most challenging clinical problems in cardiology, associated with a broad spectrum of pathological triggers and a wide range of clinical presentations that vary from mild dyspnea to acute heart failure and sudden death1, 2. Although the true incidence of myocarditis is unknown due to its difficult diagnosis and infrequent collection of endomyocardial biopsies, myocarditis is found in $9.6 \%$ of patients with unexplained heart failure ${ }^{3}$. It frequently affects young, previously healthy individuals ${ }^{4}$ and has been estimated to account for up to $12 \%$ of sudden deaths in patients under 40 years of age 5 . Although its prognosis varies by cause, five-year survival in aggregate cohorts of biopsy-proven myocarditis is approximately $50 \% 6$. Although mycarditis can also be caused by infection with bacteria, fungi, parasites, and non-infectious agents such as autoimmune disorders and drug-induced hypersensitivity, viruses are the most common cause of myocarditis in western society ${ }^{2,7}$. Viruses associated with myocarditis in humans include enteroviruses, adenoviruses, influenza viruses, cytomegaloviruses, parvoviruses, herpes viruses, and human immunodeficiency virus ${ }^{7}$. Traditionally, the enteroviral coxsackievirus B3 (CVB3) and adenoviruses have been the most prevalent pathogens found in myocarditis biopsies, but more frequent identification of parvovirus B19 and human herpes virus 6 has been observed recently, the cause of which is incompletely understood ${ }^{7-9}$. Although most of these 'cardiotropic' viruses are common viruses that cause only mild upper respiratory or gastrointestinal complaints in most individuals, their infection of the heart can, in some patients, cause direct cardiac damage followed by an inflammatory response that inflicts additional injury to the heart4. Clinical outcome is determined by the interplay between virulence, host response, and therapeutic intervention, and may range from subclinical disease to congestive heart failure with hemodynamic instability and death in the acute phase1. Subsequently, dilated cardiomyopathy (DCM) may result from chronic inflammatory activation due to incomplete clearance of cardiac viral genomes ${ }^{10-12}$, or due to an autoimmune response triggered by exposure to cardiac auto-antigens ${ }^{13-16}$. In the case of complete viral elimination with resolution of cardiac inflammation, patient outcome depends on the amount of irreversible damage inflicted to the myocardium ${ }^{17}$. Intriguingly, the observation that cardiotropic viruses are frequently found in heart tissue from patients with $\mathrm{DCM}^{8,18}$ suggests that viruses may also be hitherto unrecognized triggers for DCM by causing subclinical myocarditis.

Although viruses can directly damage cardiac cells, the reactive inflammatory response significantly contributes to cardiac injury and ensuing morbidity ${ }^{1}$. A central theme in viral myocarditis research is therefore whether cardiac damage is primarily caused by direct virus-induced injury or by immune mechanisms. In this review we discuss the evidence for the beneficial versus adverse roles of inflammation in viral myocarditis and recapitulate the lessons learned from preclinical and clinical studies about inflammation as therapeutic target for this disease. 


\section{Infection versus autoimmune disease}

There is strong clinical and experimental evidence demonstrating that viruses can directly cause cardiomyopathy ${ }^{19,20}$ and that unsuccessful viral elimination may lead to progressive disease ${ }^{4,10-12}$. Such observations support the idea that immune responses are required for defense of the virus-infected heart. By contrast, the extent of inflammation in patients with acute myocarditis is in itself an independent negative predictor of outcome ${ }^{9}$, and many preclinical studies have shown a protective effect of anti-inflammatory interventions during viral myocarditis in mice $21-23$. For example, a pioneer study by Woodruff in 1974 showed that depletion of T lymphocytes in mice by either thymectomy or anti-thymocyte serum reduced cardiac injury without affecting viral elimination ${ }^{21}$. Therefore, clearly not all inflammatory responses are beneficial and, consequently, the potential of interventions in the inflammatory response during myocarditis has been intensely studied ${ }^{24}$. While broad immunosuppression fails to improve cardiac function in myocarditis patients ${ }^{25}$, accumulating evidence from clinical and preclinical studies supports the feasibility of targeted strategies to improve disease outcome $22,26-28$. The use of CVB3 infection in mice as a model of enteroviral infection in humans ${ }^{29,30}$ (Figure 1) and our capability of genetically manipulating the mouse genome have broadened widely our understanding of the mechanisms underlying the acute and chronic pleiotropic effects of inflammation in viral inflammatory heart disease. Building on knowledge acquired from such animal models, the next sections will discuss the pathogenesis of cardiac injury during enteroviral infection, direct virus effects on myocyte homeostasis, and summarize our current understanding of the respective roles of the innate and adaptive immune system in both viral elimination and cardiac injury.
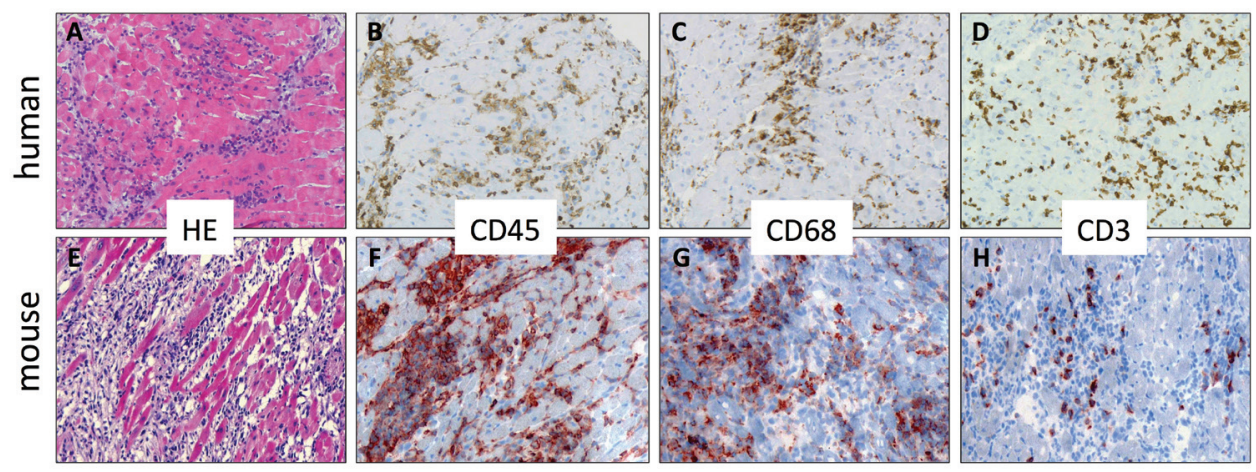

Figure 1. Immunopathological features of viral myocarditis in humans and mice. Panels $A-D$ show stainings of a right ventricular septal biopsy from a 49-year old female patient with acute viral myocarditis, presenting with acute dyspnea and soon developing cardiogenic shock due to impaired cardiac contractile function (left ventricular ejection fraction 15\%). Histological examination showed foci of myocyte necrosis (A) and infiltration of the myocardium by CD45-positive leukocytes (B) containing both CD68-positive macrophages (C) and CD3-positive T lymphocytes (D). Panels E-H show comparable cardiac injury and leukocyte infiltration in Coxsackievirus-induced myocarditis in the heart of a male adult $\mathrm{C} 3 \mathrm{H}$ mouse. Magnification: 200x. Human images were kindly provided by Dr. Bart de Vries from the Maastricht University Medical Center Pathology Department. 


\section{Pathogenesis of CVB3 induced myocarditis}

Coxsackieviruses are non-enveloped, single stranded RNA enteroviruses, belonging to the family of Picornaviridae. They are fecal-orally transmitted. While $50-80 \%$ of non-poliovirus enteroviral diseases are completely asymptomatic ${ }^{31}$, the group B coxsackieviruses (CVB) are associated with the development of myocarditis in humans, in particular serotype B34. Following uptake through the host gastrointestinal tract, CVB3 infects and replicates in lymphocytes and macrophages of Peyer's patches and the spleen ${ }^{32}$, 33. Subsequently, infectious virions are released into the bloodstream and disseminate into organs such as the heart and pancreas. Target cell internalization occurs through interaction with two wellstudied surface receptors: the decay accelerating factor (DAF) is responsible for anchoring the virion to the cell surface, after which the coxsackievirus and adenovirus receptor (CAR) - which is shared by humans and mice - mediates cellular entry ${ }^{4}$. CAR-mediated internalization is a prerequisite for the development of CVB3-induced myocarditis as CAR deficient mice are resistant to both cardiac infection and inflammation ${ }^{34}$. Following virus uncoating, the 7.4 kilobase viral genome is translated into a single polyprotein, which is cleaved into functional proteins by virus-encoded proteases. The detailed molecular biology of CVB3 infection is beyond the scope of this review but has been excellently reviewed elsewhere ${ }^{4}$.

The development of viral myocarditis is generally divided into three distinct phases (Figure 2). The first 3-4 days are the 'acute' phase, which is characterized by virus replication and interaction with myocyte signaling prior to cardiac infiltration by innate immune cells. Both direct proteolytic damage by the virus ${ }^{19}$ and recognition of viral pathogen motifs through cardiac toll-like receptors (TLRs) ${ }^{35}$ contribute to the increasing expression of pro-inflammatory cytokines including interleukin-1 $\beta$ (IL-1 $\beta$ ), IL-6, IL-18, tumor necrosis factor- $\alpha$ (TNF $\alpha$ ), and type I and type II interferons (IFNs). These cytokines are produced by cardiac resident cells, including myocytes, fibroblasts, endothelial cells, and dendritic cells (DC) ${ }^{27}{ }^{36}$. Type I interferons (IFN- $\alpha$ and IFN- $\beta$ ) are especially important in the early antiviral response and have a range of effects on infected cells. They downregulate protein synthesis, stimulate p53-mediated apoptosis ${ }^{37}$, and upregulate the immunoproteasome and major histocompatibility complex (MHC) classes I and II. In concert, these cytokine signals activate local macrophages and upregulate endothelial adhesion molecules as well as chemokines and chemokine receptors to collectively trigger the recruitment of innate immune cells ${ }^{38}$. The subacute phase, from day $4-5$ to approximately day 14 , commences with infiltration of the heart by cells of the innate immune system, including natural killer (NK) cells that are important for viral elimination by directly recognizing and killing infected cell ${ }^{39}$. In addition, monocytes engage in phagocytosis of dead cells and strongly augment the expression of pro-inflammatory cytokines. Within days, immune cells from the adaptive immune system also accumulate in the infected heart, including both $\mathrm{T}$ and $\mathrm{B}$ lymphocytes. $\mathrm{CD}^{+}$cytotoxic $\mathrm{T}$ cells directly bind and eliminate infected cells by recognizing $\mathrm{MHC}$ class I antigens on infected cardiomyocytes, assisted by the effects of TNF- $\alpha$ and IFN- $\gamma$ which promote $\mathrm{MHC}$ class I presentation and facilitate cell-cell contact between $\mathrm{T}$ cells and myocytes40. CD4 ${ }^{+} \mathrm{T}$ helper cells do not directly kill infected cells but are important 

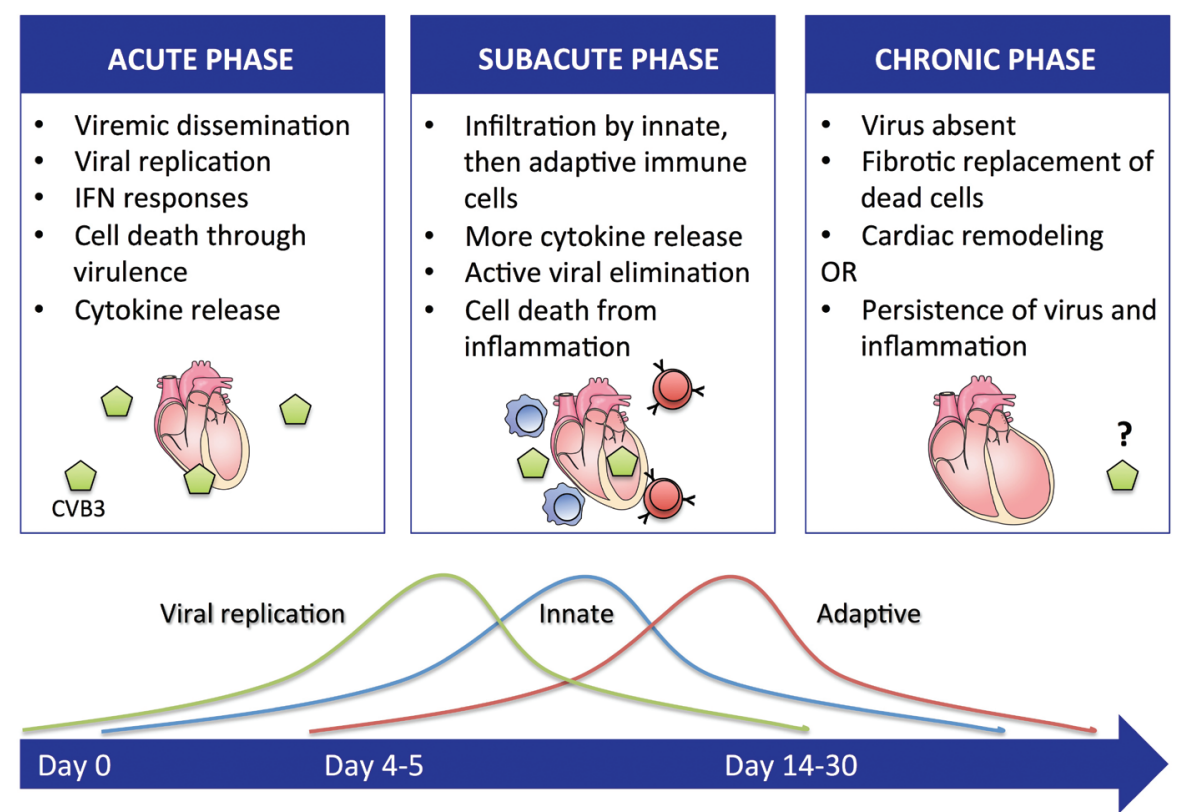

Figure 2. Pathogenesis of viral myocarditis. The pathogenesis of viral myocarditis if often divided into three phases. During the acute phase of viral myocarditis, viral infection (represented by the green pentagon) and replication in the heart cause cell death and cytokine responses from resident cells. During this phase, inflammatory signaling is largely protective to the heart. Cytokine signaling then causes an influx of immune cells (first innate, then adaptive) marking the subacute phase. These inflammatory cells engage in viral elimination but importantly contribute to cardiac injury. In the chronic phase, effective viral elimination leads to inhibition of the immune response by anti-inflammatory macrophages and regulatory $T$ cells. These cells additionally stimulate repair processes such as fibrotic remodeling. Cardiac outcome is determined by the extent of irreversible damage inflicted during the acute and subacute phases. Conversely, replicating or nonreplicating virus can persist in the myocardium causing chronic inflammation and progressive left ventricular dysfunction.

mediators between professional antigen presenting cells and cytotoxic T cells as well as B cells, which produce neutralizing antibodies to limit viral replication ${ }^{41}$. During the subacute phase, the immune response not only eliminates infected and dead cells but also significantly contributes to irreversible cardiac damage.

The third stage of viral myocarditis starts after complete elimination of the virus and is characterized by cardiac repair and remodeling. Anti-inflammatory cytokines such as transforming growth factor- $\beta$ (TGF- $\beta$ ) and IL-10 secreted by regulatory T cells and alternatively activated (M2) macrophages promote resolution of the immune response and replacement of dead tissue by a fibrotic scar ${ }^{38}$. Depending on the extent of myocardial damage, cardiac dilation and compensatory hypertrophy during this phase may contribute to contractile dysfunction in the long term ${ }^{4}$. Alternatively, failure to completely clear virus from the heart may result in chronic inflammation and accelerated progression to $\mathrm{DCM}^{10,12}$. In mice, acute viral myocarditis can result either in complete resolution of disease or chronic viral persistence and inflammation depending on the mouse strain used. Since awareness of such strain differences is helpful in tailoring experimental studies and interpreting results, they will be discussed in the next section. 


\section{Mouse models of enteroviral myocarditis}

The susceptibility to enterovirus-induced viral myocarditis varies between mouse strains (as it does between patients), both with respect to the extent of cardiac inflammation and damage invoked during the subacute phase and with respect to viral elimination and development of chronic disease ${ }^{42}$. Such strain differences are important because they can affect findings from experimental studies, and these differences are summarized in Table 1. Factors that have been associated with susceptibility include MHC haplotype ${ }^{43,44}$, the balance of Th1 versus Th2 immune responses ${ }^{45,46}$, the efficiency of DC antigen presentation ${ }^{47,48}$, and genetic variations in the DAF and IFN- $\gamma$ genomic regions ${ }^{49}$. Although all of these factors appear to influence disease severity, none of them is an exclusive determinant. For instance, the $\mathrm{MHC}$ haplotype $\mathrm{H}-2 \mathrm{~b}$ has been associated with mild susceptibility to CVB3-induced myocarditis in C57BI/6 and 129/SvJ mice, but haplotypematched A.BY/SnJ mice develop both an acute and chronic disease associated with failure to eliminate the virus ${ }^{4,42,47}$. Strains can also differ in the cardiospecificity of disease. $\mathrm{C} 3 \mathrm{H}$ / $\mathrm{HeJ}$ mice develop severe myocardial lesions while survival is high due to minimal systemic disease, whereas C57BI/6 mice generally show a higher mortality despite limited cardiac damage, possibly associated with systemic pathology (unpublished observations). The enhanced susceptibility to viral myocarditis in males compared to females is well documented and will be discussed in more detail later in this review $23,45,50$.

Differences in CVB3 strains used may also impact experimental data ${ }^{51}$. For instance, although the widely used Nancy strain of $\mathrm{CVB}^{52}$ results in cardiac disease that is highly similar to the originally patient-propagated Woodruff $(\mathrm{H} 3)$ strain ${ }^{53}$, there may be subtle differences between the two, as it has been suggested that pathogenicity in from $\mathrm{H} 3$ strain may be more dependent on IFN- $\gamma$-mediated $\mathrm{CD}^{+} \mathrm{T}$ cell responses while reduction of IFN- $\gamma$ signaling in Nancy-strain CVB3 disease is not beneficial, possibly due to more direct viral

Table 1. Mouse strain susceptibility to the development of viral myocarditis

\begin{tabular}{lcccc}
\hline Mouse strain & H-2 haplotype & Susceptibility & Viral elimination & Reference \\
\hline C57BI/6 & b & low & yes & Chow et al. ${ }^{42}$ \\
$129 / \mathrm{S} v J$ & bc & low & yes & Zaragoza et al. ${ }^{68}$ \\
A.BY/SnJ & b & high & no & Klingel et al. ${ }^{12}$ \\
A/J & a & severe & yes & Chow et al. ${ }^{42}$ \\
C3H/HeJ & k & severe & yes & Chow et al. ${ }^{42}$ \\
BALB/C & d & high & yes & Chow et al. ${ }^{42}$ \\
CD1 & ND & yes & Godeny et al. ${ }^{39}$ \\
DBA/1J & high & no & Klingel et al. ${ }^{12}$ \\
DBA/2 & high & high & yes & Lee et al. ${ }^{29}$ \\
SWR/J & d & high & no & Klingel et al. ${ }^{12}$ \\
A.CA/SnJ & high & no & Klingel et al. ${ }^{12}$ \\
\hline
\end{tabular}

a ND, not determined 
pathogenicity ${ }^{54}$. Finally, the encephalomyocarditis virus (EMCV) has also been used to study Picornaviral myocarditis in a smaller but significant number of studies as a histopathologically comparable model to CVB3 infection ${ }^{55}$.

Different mouse models of myocarditis resemble different aspects of human disease. Mice that develop acute myocarditis followed by complete viral clearance and resolution of inflammation are well suited to study inflammatory components during the acute phase in human patients. However, many patients present after the acute phase and their management may require the use of different mouse models. For instance, lowgrade inflammation associated with viral persistence is observed in a significant proportion of patients and this phenomenon may be better investigated in mice that fail to eliminate virus (Table 1). In addition, chronic autoimmune responses directed at selfantigens that are temporarily exposed during the acute viral phase are well mimicked by the experimental autoimmune myocarditis (EAM) model. Integration of lessons learned from these different models will be important to identify suited therapeutic targets in different patients (acute versus chronic, virus positive versus negative) at different time points.

\section{Determinants of cardiac injury in viral myocarditis}

While cardiac viral replication and persistence are associated with the development and progression of myocarditis ${ }^{1,10,12}$, the relative contributions of virus and immune response to myocardial tissue destruction have long been debated. This debate is rooted in contrasting findings from early experimental studies of viral myocarditis in immune compromised mice. Woodruff and Woodruff reported in 1974 that T cell-deficient mice were protected from cardiac injury during CVB3 infection, suggesting that the $T$ cell response contributes to adverse cardiac damage without being required for viral elimination ${ }^{21}$. By contrast, Chow et al. later found that severe combined immunodeficiency (SCID) mice, which lack both $B$ and $T$ cells, display aggravated disease with increased mortality, cardiac damage, and viral titers ${ }^{20}$, providing evidence for CVB3 virulence as an important causal factor in myocarditis. Although damage from uninhibited viral activity may not accurately reflect the viral contribution to myocarditis in an immune competent host ${ }^{31}$, these studies have ignited extensive research into the contributions of components from both the innate and adaptive immune system. In this section we describe the available evidence for direct cytopathic effects from CVB3, as well as for the roles of inflammatory activation during the course of viral myocarditis. A comprehensive overview of experimental manipulations to the immune system and associated phenotypes is provided in Table 2.

\section{Direct virus-mediated cardiac injury}

In line with the aggravated damage observed in SCID animals, the potential of CVB3 to directly damage myocardial cells has clearly been demonstrated in vivo and in vitro ${ }^{4} 56$. Microscopically, infection of the virus leads to cardiomyocyte vacuolation, apoptosis, and necrosis before the infiltration of inflammatory cells ${ }^{57}$. Also, transgenic overexpression of 
replication-deficient CVB3 in the mouse heart leads to low-grade degeneration of myocytes and cardiac dilation ${ }^{58}$. Following entry into the cellular cytosol, CVB3 translation leads to the release of viral proteases. These proteases manipulate cellular homeostasis, for instance by inhibiting host protein synthesis, and damage structural proteins. A well-known example is cleavage of the cytoskeletal protein dystrophin and the dystrophin-associated glycoproteins $\alpha$-sarcoglycan and $\beta$-dystroglycan by the enteroviral protease $2 A^{19}$, 59. Dystrophin plays an important role in myocardial integrity by connecting the muscle fibers to the extracellular matrix. In line with these findings, transgenic mice with cardiac overexpression of protease 2A develop spontaneous $\mathrm{DCM}^{60}$. Finally, myocyte apoptosis, induced by either host interferon responses or virus signaling to release progeny at the end of the infectious cycle, can contribute to myocyte loss in the acute phase of myocarditis ${ }^{4}$.

\section{The innate immune response}

The innate immune system provides a fast, non-specific response to tissue damage and infections. It recognizes pathogen-associated molecular patterns by either circulating proteins of the complement system, which neutralize extracellular pathogens, or by cellular TLRs, leading to activation of downstream signaling. Non-immune cells and innate immune cells (including monocytes-macrophages, DCs, and NK cells) interact with each other through a wide variety of cytokines including type I and II IFNs, which have potent antiviral effects. Most of these aspects have been experimentally manipulated with widely varying protective or detrimental effects in the context of viral myocarditis.

Complement proteins, as immediate effectors of innate antimicrobial defense, appear to have protective effects on the heart. Mice deficient for complement protein 1 and 2 receptors display increased cardiac damage and macrophage infiltration during CVB3 infection ${ }^{61}$, whereas $\mathrm{C} 3$ is required for the rapid formation of splenic antiviral germinal centers $^{62}$. NK cells are also important for cardiac protection by selectively killing CVB3 infected cells, thus limiting CVB3 infection ${ }^{63}$, and depletion of NK cells prior to CVB3 infection leads to increased viral titers in the heart and aggravated myocyte degeneration ${ }^{39}$. Similarly, natural killer T (NKT) cells - an innate subset of T cells that are important for early antiviral responses - are also beneficial in the early phase; administration of an activating ligand for invariant NKT cells suppresses viral levels and inflammation in the heart through an early increase in IFN- $\gamma$ activity ${ }^{64}$. By contrast, broad depletion of innate immune cells by administering antibodies against Mac-1 (expressed on monocytes, macrophages, and NK cells) reduces both viral levels and myocardial inflammation after encephalomyocarditis virus (EMCV) infection ${ }^{65}$, indicating that innate immune cells other than NK cells may not be equally beneficial to the host. Macrophages, which represent the most abundant cells in inflammatory lesions during the acute phase ${ }^{12}$, may account for part of this effect. It was recently shown that classically activated (M1) macrophages - which produce abundant pro-inflammatory cytokines including TNF- $\alpha$ and IL-12 ${ }^{66}$ - are important contributors to cardiac inflammation and injury during myocarditis in BALB/c mice ${ }^{23}$. Hearts of male animals (who are more susceptible than females) display higher proportions of M1 macrophages than female hearts. Adoptive transfer of M1 polarized macrophages into female mice significantly increased the female inflammatory response to infection. 
Table 2. Phenotypic effects of experimental immune manipulations during viral myocarditis $\mathrm{s}^{\mathrm{a}, \mathrm{b}, \mathrm{c}}$

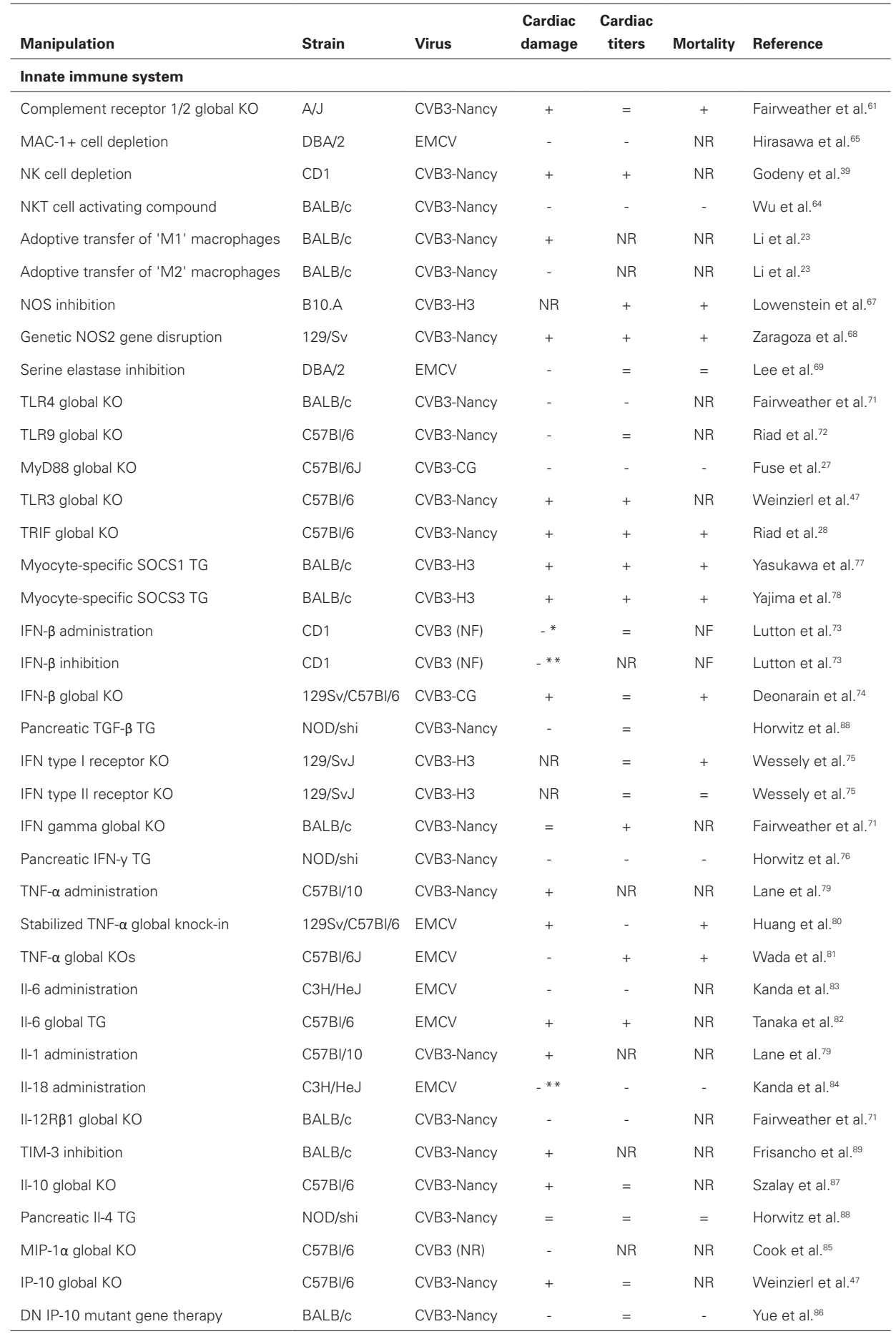


follow up of table 2

\begin{tabular}{|c|c|c|c|c|c|c|}
\hline Manipulation & Strain & Virus & $\begin{array}{l}\text { Cardiac } \\
\text { damage }\end{array}$ & $\begin{array}{c}\text { Cardiac } \\
\text { titers }\end{array}$ & Mortality & Reference \\
\hline uPA global KO & $\mathrm{C} 57 \mathrm{BI} / 6$ & CVB3-Nancy \# & - & $=$ & NR & Heymans et al. ${ }^{90}$ \\
\hline AdV-TIMP1 & C57BI/6 & CVB3-Nancy \# & - & NR & NR & Heymans et al. ${ }^{90}$ \\
\hline AdV-PAl-1 & C57BI/6 & CVB3-Nancy \# & - & NR & NR & Heymans et al. ${ }^{90}$ \\
\hline MMP-2 global KO & C57BI/6 & CVB3 (NR) & + & + & + & Westermann et al. ${ }^{92}$ \\
\hline MMP-9 global KO & 129/SvEv & CVB3-CG & + & + & $=$ & Cheung et al. ${ }^{91}$ \\
\hline MMP-8 global KO & $\mathrm{C} 57 \mathrm{BI} / 6$ & CVB3-CG & $=$ & $=$ & $=$ & Cheung et al..$^{91}$ \\
\hline \multicolumn{7}{|l|}{ Adaptive immune system } \\
\hline Thymectomy/antithymocyte serum & $\mathrm{BALB} / \mathrm{c}$ & CVB3-H3 & - & $=$ & NF & Woodruff et al. ${ }^{21}$ \\
\hline Athymic nude mice & $\mathrm{BALB} / \mathrm{c}$ & CVB3-Nancy & - & $=$ & $=$ & Hashimoto et al..$^{99}$ \\
\hline Athymic nude mice & $\mathrm{BALB} / \mathrm{c}$ & CVB3 (NF) & $=$ & $=$ & NF & Robinson et al. ${ }^{100}$ \\
\hline Athymic nude mice & NFR & CVB3 (NF) & $=$ & - & NF & Schnurr et al. ${ }^{101}$ \\
\hline SCID & $\mathrm{C} 3 \mathrm{H} / \mathrm{HeSnJ}$ & CVB3-CG & + & + & + & Chow et al..$^{20}$ \\
\hline CD4 KO & $A / J$ & CVB3-CG & $=$ & $=$ & $=$ & Opavsky et al. ${ }^{102}$ \\
\hline CD8 KO & $A / J$ & CVB3-CG & $=$ & $=$ & $=$ & Opavsky et al. ${ }^{102}$ \\
\hline CD4/8 double KO & $\mathrm{A} / \mathrm{J}$ & CVB3-CG & - & $=$ & - & Opavsky et al. ${ }^{102}$ \\
\hline TCR $\beta$-chain KO & $A / J$ & CVB3-CG & - & $=$ & - & Opavsky et al. ${ }^{102}$ \\
\hline Perforin KO & $\mathrm{C} 57 \mathrm{BI} / 6$ & CVB3-H3 & - & $=$ & - & Gebhard et al. ${ }^{104}$ \\
\hline Perforin global KO & C57BI/6 & CVB3-Nancy & $=$ & $=$ & $=$ & Klingel et al. ${ }^{103}$ \\
\hline Fas global KO & $\mathrm{BALB} / \mathrm{c}$ & CVB3-H3 & - & NR & NR & Huber et al. ${ }^{106}$ \\
\hline FasL global KO & $\mathrm{BALB} / \mathrm{c}$ & CVB3-H3 & - & NR & NR & Huber et al. ${ }^{106}$ \\
\hline үठ T cell depletion & $\mathrm{C} 57 \mathrm{BI} / 6 * * *$ & CVB3-H3 & - & - & NR & Huber et al. ${ }^{107}$ \\
\hline Adoptive transfer of T cells F-to-M & $\mathrm{BALB} / \mathrm{c}$ & CVB3-H3 & - & $=$ & NR & Huber et al. .5 \\
\hline MHC class II global KO & $\mathrm{C} 57 \mathrm{BI} / 6$ & CVB3 (NR) & - & + & NR & Leipner et al. ${ }^{110}$ \\
\hline$\beta-2$ microglobulin global $\mathrm{KO}$ & $\mathrm{C} 57 \mathrm{BI} / 6$ & CVB3-Nancy & + & + & $=$ & Klingel et al. ${ }^{103}$ \\
\hline CD1d global KO & $\mathrm{BALB} / \mathrm{c}$ & CVB3-H3 & - & $=$ & $=$ & Huber et al..$^{126}$ \\
\hline $\mathrm{B}$ cell $\mathrm{KO}$ & C57BI/6 & CVB3-Nancy & + & + & NR & Mena et al. ${ }^{111}$ \\
\hline Adoptive transfer of regulatory $T$ cells & $\mathrm{C} 57 \mathrm{BI} / 6$ & CVB3 (CG) & - & - & - & Shi et al. ${ }^{22}$ \\
\hline Estradiol administration to males & $\mathrm{C} 57 \mathrm{BI} / 6 * * *$ & CVB3-H3 & - & - & NR & Huber et al. ${ }^{107}$ \\
\hline Testosterone administration to females & $\mathrm{C} 57 \mathrm{BI} / 6 * * *$ & CVB3-H3 & + & + & NR & Huber et al. ${ }^{107}$ \\
\hline Male gonadectomy & BALB/c & CVB3-Nancy & - & $=$ & NR & Frisancho et al. ${ }^{50}$ \\
\hline
\end{tabular}

"+", "=", and "-" signify increases, no changes, and decreases, respectively

Abbreviations: NR not reported; NF not found; KO knockout; TG transgenic mouse; DN dominant negative; AdV adenoviral overexpression; F-to-M female to male; CVB3-CG Charles Gauntt strain (propagated from and closely related to the H3 strain)

* Only when treated early in disease

** Only when treated late in disease

*** Transgenic C57BI/6 strain (B1.Tg.E $\alpha$ ) expressing MHC class II IE

\# Lipopolysacharide (LPS) was also administered as adjuvant stimulus

General comment: many components of the immune system are not exclusive to either innate or adaptive responses. We have made a rough separation for heuristic purposes only 
By contrast, transfer of M2 macrophages, which secrete anti-inflammatory cytokines such as IL-1066, into male animals inhibited cardiac disease, with non-significant effects on viral levels ${ }^{23}$. Importantly, the detrimental effects of innate cells on the myocardium are not mediated by the toxicity of nitric oxide (NO), which is secreted in large quantities by macrophages and neutrophils, because pharmacological or genetic targeting to reduce the concentration of $\mathrm{NO}$ both result in increased viral titers and aggravated disease ${ }^{67,68}$. By contrast, serine elastase activity from leukocyte infiltration has been shown to mediate adverse effects and be a potential therapeutic target ${ }^{69}$.

There is strong evidence that the activation of distinct TLR signaling cascades following pathogen recognition leads to beneficial or adverse immune responses ${ }^{35}$ (Figure 3).

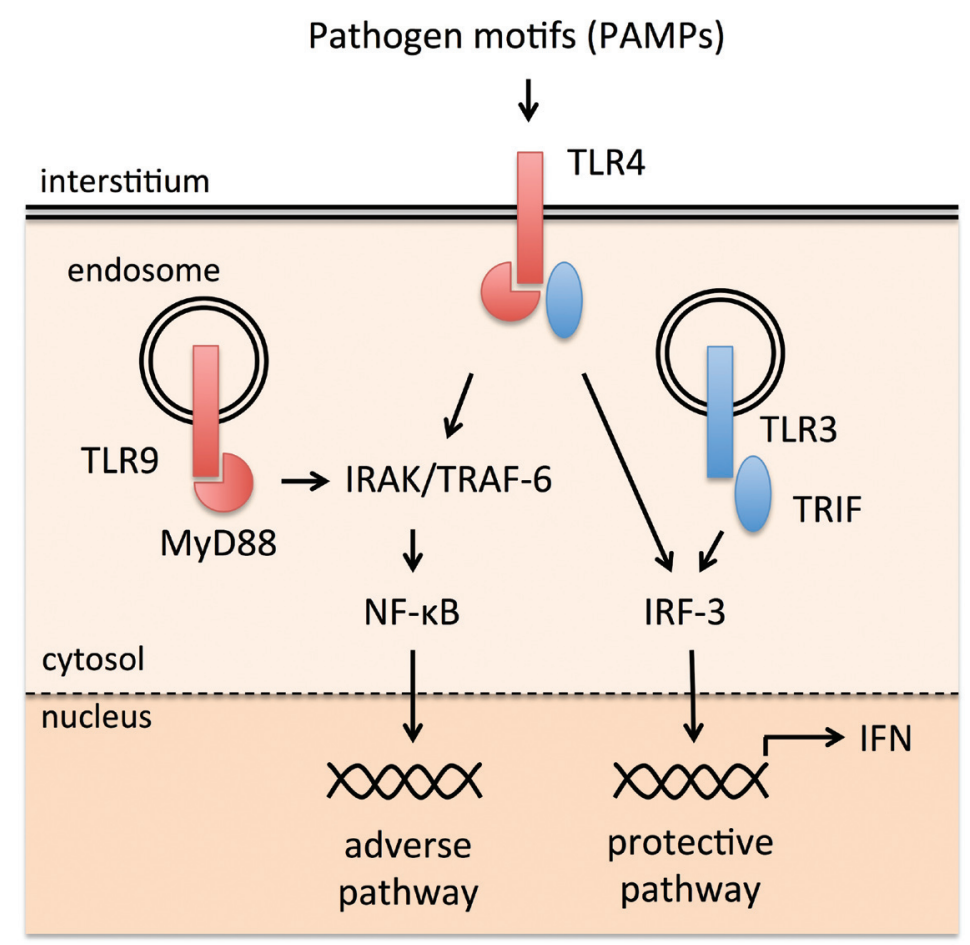

Figure 3. Effects of toll-like receptor (TLR) signaling pathways in viral myocarditis. Schematic representation of signaling from selected TLRs after PAMP (pathogen associated molecular pattern) recognition. TLR4 and TLR9 signaling contribute to disease severity in mouse viral myocarditis through their common adapter protein MyD88. TLR4 is located on the cell membrane and recognizes lipopolysacharide (LPS) as well as several viruses. TLR9 recognizes unmethylated CpG motifs on bacterial or viral DNA in endosomes. Signaling through the MyD88-dependent pathway initiates a signal transduction cascade involving TNF receptor-associated factor 6 (TRAF-6) and Interleukin-1 associated kinase (IRAK) that ultimately leads to nuclear factor kappa B (NF-kB) activation, which may contribute to the inflammatory response. Alternatively, signaling through TLR3 is protective in viral myocarditis. TLR3 is the only TLR that does not signal through MyD88 but through TIR domain-containing adapter-inducing interferon- $\beta$ (TRIF), which promotes type I and II interferon responses via interferon regulatory factor 3 (IRF-3) and is necessary for adequate antiviral defense in myocarditis. 
Of the 10 TLRs shared by humans and mice, the majority signal through a common downstream adaptor molecule called myeloid differentiation primary response gene 88 (MyD88), which typically leads to activation of nuclear factor kappa B (NF-kB) ${ }^{35}$. An important exception is TLR3, which recognizes double-stranded RNA and signals through TIR-domaincontaining adapter-inducing interferon- $\beta$ (TRIF), which activates interferon regulatory factor 3 (IRF3) to upregulate both type I and type II IFN expression $35,70$.

Although not all TLRs have been investigated in knockout models, both TLR4- and TLR9deficient mice are protected against CVB-induced cardiac injury with uncompromised viral clearance ${ }^{71,72}$. In agreement with these studies, deficiency of the common adaptor protein MyD88 leads to nearly absent inflammation in combination with suppressed viral levels in cardiac tissue ${ }^{27}$. These data indicate that the effects of the MyD88-mediated TLR signaling pathways contribute to cardiac pathology while bearing little importance for viral elimination. By contrast, viral replication and cardiac damage are aggravated in TLR3 knockout animals ${ }^{47}$. ${ }^{70}$, and transgenic overexpression of TLR3 decreases mortality even in type I IFN deficient animals through stimulation of type II IFN signaling ${ }^{70}$. In agreement, mice lacking the TLR3adaptor TRIF have impaired type I IFN responses and develop severe myocarditis with high viral loads, resulting in $100 \%$ mortality 28 .

\section{Cytokines in the innate immune response}

In line with the central role of IFNs in antiviral responses, administration of IFN- $\beta$ reduces myocardial lesions when given in the acute stage of CVB3-induced myocarditis ${ }^{73}$. Conversely, inhibiting IFN- $\beta$ ameliorates disease only when given in a later stage, suggesting that the favorable effects of type I IFN may be time-dependent ${ }^{73}$. Survival is markedly reduced in mice lacking either the IFN- $\beta$ gene ${ }^{74}$ or the type I IFN receptor ${ }^{75}$ (thus deficient in both IFN- $\alpha$ and IFN- $\beta$ signaling). Interestingly, although IFN- $\beta$ knockouts also display increased myocardial damage, cardiac viral levels are unchanged in both knockouts despite dramatic viral replication in the liver, possibly causing the observed mortality. Mortality and viral titers were not affected in IFN- $\gamma$ deficient animals ${ }^{75}$, although pancreatic overexpression of IFN- $\gamma$ protects against cardiac damage, viral replication, and mortality following CVB3 infection ${ }^{76}$. IFN effects are partly mediated through the janus kinase (JAK)-signal transducer and activator of transcription (STAT) signaling pathway, and myocyte-restricted transgenic overexpression of either of two key inhibitors of this pathway, suppressor of cytokine signaling-1 (SOCS-1) and SOCS-3, markedly augments cardiac damage and viral replication, showing that JAK-STAT signaling is important for myocyte resistance to $\mathrm{CVB}^{77,78}$. Aiming to translate the beneficial effects of IFN signaling to the patient setting, a phase II pilot study reported that 6 months of IFN- $\beta$ administration to 22 patients with persistent left ventricular (LV) dysfunction and viruses in LV biopsies resulted in complete viral clearance and improved LV function ${ }^{26}$. Although promising, these results await confirmation in randomized controlled studies ${ }^{17}$.

In addition to IFNs, a broad range of pro- and anti-inflammatory cytokines have been functionally studied as treatment targets in the context of viral myocarditis; their effects are often dose- and time-dependent. For example, exogenous TNF- $\alpha$ administration during CVB3 infection aggravates myocardial damage ${ }^{79}$, and transgenic mice bearing a genetically 
stabilized form of TNF- $\alpha$ (which leads to enhanced TNF expression and activity) display increased cardiac damage after EMCV infection, albeit with enhanced viral clearance ${ }^{80}$. However, systemic TNF- $\alpha$ deficiency also leads to more cardiac injury and mortality after EMCV infection, which can be rescued by exogenous administration of TNF- $\alpha$ during the acute phase of disease ${ }^{81}$. Collectively, these studies demonstrate that TNF- $\alpha$ is an antidote for cardiac viral infection but with the need for accurate dosing, given that either insufficient or exaggerated expression of TNF- $\alpha$ is equally harmful. Dose- and time-dependency were also observed for IL-6, as transgenic overexpression of IL-6 aggravates the course of EMCV myocarditis ${ }^{82}$ whereas recombinant IL-6 administration in the acute, but not subacute phase, is protective ${ }^{83}$. Similarly, beneficial effects of IL-18 treatment (involving enhanced NK cell activity) were only observed from treatment at the time of infection, whereas initiation of treatment at day 2 post-infection failed to provide cardioprotection ${ }^{84}$. Detrimental roles have been ascribed to two other pro-inflammatory cytokines, IL-1 and IL-12. IL-1 administration increases cardiac damage during CVB3 infection ${ }^{79}$ and mice deficient for the IL-12 receptor have dramatically reduced damage and cardiac viral loads ${ }^{71}$. Chemokines have diverging effects during myocarditis: myocardial damage is reduced in knockout mice for macrophage inflammatory protein-1 $\alpha$ (MIP-1 $\alpha$, also known as CCL3) $)^{85}$ as well as in animals treated with inhibitors for IFNy-induced protein-10 (IP-10, also known as CXCL10) ${ }^{86}$. However, damage is increased in animals systemically deficient for IP-10, and more research is required to draw conclusions about the roles of chemokines during viral myocarditis ${ }^{47}$.

In general, anti-inflammatory cytokines have been associated with the attenuation of viral myocarditis. For instance, IL-10 knockout animals fail to resolve the inflammatory response after the acute phase leading to chronic disease and cardiac injury ${ }^{87}$. Myocarditis is also inhibited by pancreatic overexpression of TGF- $\beta$, although surprisingly not by the antiinflammatory IL-488. Also, expression of immunomodulatory T cell Ig mucin-3 (TIM-3) during the innate immune response is important for controlling adverse inflammation, partly through cross-talk with the adaptive immune system ${ }^{89,90}$.

A final molecular class that merits mentioning are the matrix metalloproteinases (MMPs); although not exclusively part of the immune system, they exert critical functions in the inflammatory response, including matrix degradation and facilitation of leukocyte migration, as well as cytokine processing and regulation ${ }^{38}$. Many MMPs are upregulated during viral myocarditis and seem to be net detrimental, given that inflammation and fibrosis are reduced by broad inhibition of MMP activity in mice deficient for the MMP-activating urokinase-type plasminogen activator (UPA) or viral overexpression of endogenous inhibitors tissue inhibitor of metalloproteinases-1 (TIMP-1) and plasminogen activator inhibitor-1 (PAI1) ${ }^{90}$. Surprisingly, mice deficient in single MMPs have yielded contrasting findings. Whereas inflammation and viral load is not affected in MMP-8 knockouts, mice lacking MMP-9 have increased myocardial damage and dysfunction associated with higher viral loads and infiltration ${ }^{91}$. Similar findings were recently reported for MMP-3, which seems related to increased expression of the macrophage chemoattractant protein-3 (MCP-3) ${ }^{92}$. Further research is required for a better understanding of the role of MMPs during viral myocarditis. 


\section{The adaptive immune response}

The adaptive immune system responds to specifically recognized antigenic epitopes through clonal expansion of antigen-specific $\mathrm{T}$ and $\mathrm{B}$ lymphocytes, causing cellular and humoral antibody-mediated immune responses, respectively. Pathogen neutralization by the adaptive immune system is more sophisticated than during the innate response, and its contribution to cardiac injury is more complex. Although CD8 ${ }^{+} \mathrm{T}$ lymphocytes directly contribute to myocyte death by killing infected cells, there is strong evidence that the generation of autoimmune reactivity during virus-induced inflammation may significantly contribute to disease severity ${ }^{46,93}$. For instance, following early antiviral antibody production, the accumulation of antibodies directed at cardiomyocyte constituents, such as myosin, is a common finding in both CVB3-infected mice and myocarditis patients ${ }^{94,95}$. Factors that influence the likelihood and severity of self-reactivity include the duration of viral antigenic exposure ${ }^{17}$. In addition, Th1 signaling favoring IFN- - -mediated cellular immune responses seems to promote autoimmune cardiac damage as compared with Th2 signaling which favors antibody-mediated immune responses45. Differential propensities to Th1 versus Th2 responses have been shown to account for variable susceptibility across different mouse strains, as well as differential responses based on gender 44,45 .

Auto-reactive responses during viral myocarditis develop in different ways ${ }^{93}$. Increased exposure to auto-antigens from dying cells can cause activation of low-affinity self-reactive clones that have evaded negative selection during lymphocyte maturation. Alternatively, similarity between viral epitopes and host proteins leads to cross-reactivity, a process called molecular mimicry. Whereas antibodies against CVB3 can cross-react with cardiac myosin and aggravate viral myocarditis in mice ${ }^{96}$, the parallel presence of autoantibodies directed against a range of host proteins is more in agreement with pathogenetic mechanisms of auto-exposure ${ }^{93}$. Concordantly, injection of apoptotic cardiomyocytes in combination with lipopolysaccharide (LPS) stimulation is sufficient to provoke myocarditis ${ }^{97}$. In fact, the most frequently used models of experimental autoimmune myocarditis (EAM) involve injecting either dendritic cells loaded with a self antigen (typically myosin) or free myosin into mice in combination with appropriate adjuvants that stimulate TLRs and T cell receptors ${ }^{93,97,98 .}$ As previously mentioned, it has long been known that mice deficient in T cells develop milder myocarditis irrespective of viral levels ${ }^{21}$. Several studies in athymic nude mice subsequently showed either cardiac protection or no change in disease, suggesting that T cells are at least not essential to CVB3 defense, if not detrimenta|99-101. These observations were followed by a more detailed study of $T$ cell subsets by Opavsky and colleagues, using mice deficient in CD4, CD8, or both co-receptors ${ }^{102}$. Whereas the phenotypes of single CD4 and CD8 knockout mice were grossly unaffected, survival and cardiac histology were markedly improved in CD4/CD8 double knockouts. Viral loads were unaffected in all of the strains. In addition, deficiency of the $T$ cell receptor beta chain was equally protective as CD4/CD8 deficiency ${ }^{102}$. An important insight from this study was that both CD4 and CD8 cells contribute to adverse inflammation and that each can compensate for deletion of the other.

Given the prominent role of T cells in the pathogenesis of viral myocarditis, two subsequent studies have evaluated the role of perforin, the main cytotoxic effector molecule of CD8 ${ }^{+}$ cytotoxic cells. Their findings are slightly discrepant. Whereas Klingel et al. found no 
differences in myocarditis, viral load, and mortality between perforin knockout and wild-type mice ${ }^{103}$, Gebhard and colleagues reported improved survival and cardiac histology in perforin deficient animals ${ }^{104}$. Although the reasons for these discrepancies are unclear, both studies are consistent with the borderline protection observed in CD8 knockout animals ${ }^{102}$. Also, both indicate that perforin is not vital for an adequate immune response in enteroviral myocarditis.

A series of papers from Huber et al. has indicated that $\gamma \delta T$ cells (a subset of $T$ cells expressing the $\gamma \delta T$ cell receptor) may be central mediators of myocarditis susceptibility, as they are enriched in lymphocytes isolated from myocarditis mouse hearts ${ }^{105}$. Activity of $\gamma \delta$ T cells promotes cardiac damage through Fas-mediated killing of myocytes and Th2 cells, hence polarizing the cell population towards Th1 responses ${ }^{105}{ }^{106}$, whereas depletion of $\gamma \delta$ T cells results in a shift towards Th2 signaling that results in disease resistance ${ }^{44,107 .}$

An effective immune response requires the adequate temporal and spatial regulation of inflammatory activity. Within the adaptive immune system, $\mathrm{CD} 4^{+} \mathrm{CD} 25^{+} \mathrm{FOXp} 3^{+}$regulatory $T$ cells fulfill this function by secreting anti-inflammatory cytokines such as IL-10 and dampening Th1 responses ${ }^{108}$. Regulatory $T$ cells are efficiently induced in the heart by CVB3 infection ${ }^{109}$, and adoptive transfer of regulatory $T$ cells to BALB/C mice prior to CVB3 infection reduces mortality, histological injury, and cardiac viral titers compared with naïve $\mathrm{T}$ cells and PBS controls ${ }^{22}$, identifying regulatory $T$ cells as important modulators of inflammation with a beneficial role in viral myocarditis.

In addition to isolated $T$ cell subset studies, the involvement of adaptive immunity has been addressed in a broader fashion. Interestingly, MHC class II knockout mice display diminished subacute inflammation similar to $\mathrm{T}$ cell deficient mice, but this is associated with a lack of neutralizing antibodies, viral persistence, and chronic disease with marked cardiac fibrosis ${ }^{110}$. In agreement with this finding, it has been proposed that effective MHC class Il-antigen presentation by DC may be an important determinant of resistance against viral myocarditis ${ }^{47}$. Strong evidence for the beneficial importance of lymphocyte responses was also provided by the inability of severely immune deficient SCID mice to control viral replication, leading to both increased myocarditis and mortality ${ }^{20}$. It is conceivable that differential B lymphocyte defects may explain some of the discrepancies between these models. Consistent with this notion, B cell knockout animals fail to eliminate virus throughout their lifetime and develop severe inflammatory pathology over time ${ }^{111}$.

\section{Interplay between viral replication and inflammation}

Table 2 summarizes the details and outcomes of inflammatory manipulations during viral myocarditis. From this table emerges a striking and perhaps surprising correlation between reported cardiac inflammatory changes and cardiac viral loads. Although it might be hypothesized that attenuating inflammatory activity could allow for increased viral replication, it is the opposite that occurs. From 46 studies that primarily intervened in the immune response and reported on both cardiac inflammation (increased or attenuated) and viral titers, 25 (54\%) observed homodirectional viral load changes, 18 
(39\%) did not find differences in viral titers, and only 3 (7\%) reported viral load changes to be inversely correlated to the level of myocarditis. These paradoxical findings are consistent with the view that many immune activities are not required for viral elimination. They also indicate that inflammatory signaling and viral replication may be closely intertwined ${ }^{112}$. Indeed, inflammatory signaling environments support virus-driven cellular signaling for successful replication ${ }^{113-116}$. For example, p38 mitogen-activated protein kinases (p38 MAPK) are a class of stress-responsive kinases that mediate cellular signaling responses to a variety of stress-stimuli such as cytokines ${ }^{117}{ }^{118}$. CVB3 infection also causes p38 MAPK activation, which is required for viral replication ${ }^{116}$, and inhibition of p38 MAPK signaling impairs viral replication both in vitro and in vivo ${ }^{113}$. An addition, ERK signaling promotes enteroviral replication in both T cells and cardiomyocytes ${ }^{4}$. Such observations may reflect a viral strategy of adapting to host signaling and help explain the positive correlation between inflammatory activity and viral loads. In addition, because viral replication in hematopoietic cells seems important, at least in the early infectious phase, immune signaling may directly affect early CVB3 replication in leukocytes to impact remote viral titers ${ }^{33,111,119}$.

\section{Gender differences in viral myocarditis}

The impact of gender on susceptibility to viral myocarditis is widely recognized, both in humans and mice. Myocarditis more frequently affects men versus women ${ }^{1,2}$. Also, CVB3 infection in mice leads to more severe inflammation and lesions in males compared with female mice ${ }^{45}$. Several explanations have been proposed to explain such differences. Huber et al. reported in 1994 that infiltrates in male BALB/c mice contained many lymphocytes of the Th1 subtype, whereas Th2 cells were more predominant in female lesions ${ }^{45}$. Moreover, treatment of male mice with estrogen increased the frequencies of IL-4 producing Th2 cells and conferred myocarditis resistance, whereas testosterone administration to female mice enhanced their susceptibility and cardiac accumulation of IFN- $\gamma$ expressing Th1 cells ${ }^{45}$. A follow-up study suggested that the differential Th1/Th2 response may in part be explained by the polarizing effects of $\gamma \delta$ T cells, which were more abundant in male and testosterone-treated female mice ${ }^{107}$. Indeed, IFN- $\gamma$ deficient females develop more myocarditis and higher viral titers than males ${ }^{54}$, confirming the importance IFN- $\gamma$ signaling mediated effects for gender susceptibility. In addition to IFN- $\gamma$, elevated TLR4 levels in male versus female mouse lesions have also been linked to the diverging immune response, as well as the higher abundance of anti-inflammatory mediators (such as TIM-3, IL-4, and regulatory T cells) in females ${ }^{120}$.

Macrophage activity also affects sex-related susceptibility. Macrophages in the inflammatory infiltrates of male BALB/c mice are predominantly of the classically activated M1 subtype, associated with high expression of pro-inflammatory cytokines including IL-12 and TNF ${ }^{23}$. By contrast, female mice have large numbers of anti-inflammatory M2 macrophages. Adoptive transfer of M2 macrophages ameliorates myocarditis in male mice whereas M1 macrophages aggravate inflammation in females, convincingly demonstrating the 


\section{PROTECTIVE}

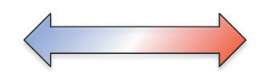

ADVERSE

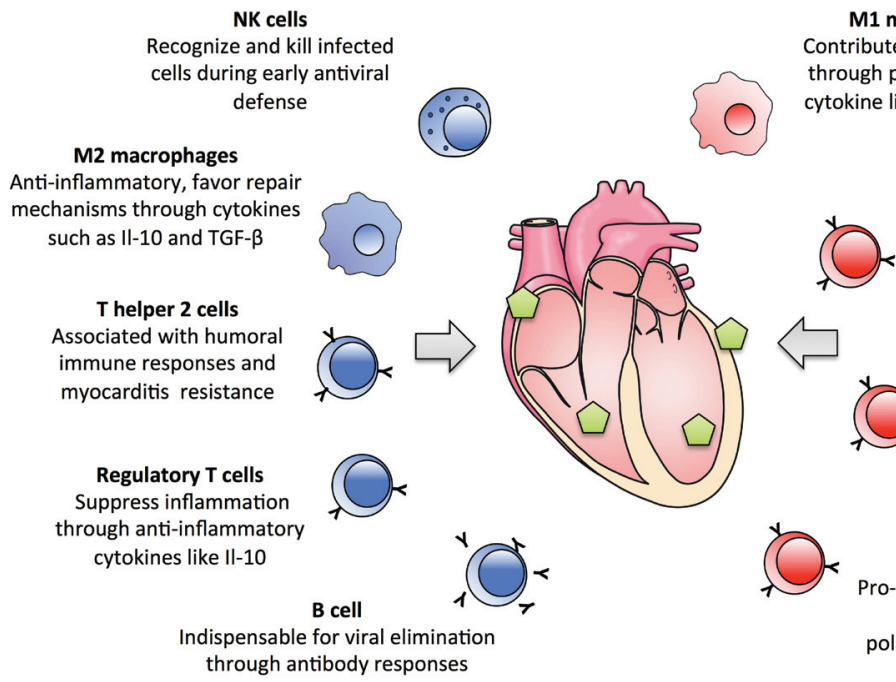

ecognize and kill infected

defense

M2 macrophages

ti-inflammatory, favor repair echanisms through cytokines

such as II-10 and TGF- $\beta$

Thelper 2 cells

Associated with humoral

mmune responses and

M1 macrophages

Contribute to cardiac injury

through pro-inflammatory

cytokine like TNF- $\alpha$ and II-6

Figure 4. Protective and adverse cellular factors in viral myocarditis. Different leukocyte subsets have divergent effects on myocarditis severity in mice. Predominantly protective cell types include natural killer (NK) cells, alternatively activated (M2) macrophages, T helper 2 (Th2) cells, regulatory T cells, and B cells. NK cells are important for viral elimination and B cells through neutralizing antibody production. M2 macrophages, Th2 cells and regulatory T cells have 'anti-inflammatory' properties, which disfavor cellular immune responses leading to tissue injury. Leukocyte subsets with predominantly adverse effects include classically activated (M1) macrophages, Th1 cells, cytotoxic T cells and $\gamma \delta T$ cells. These cells contribute to pro-inflammatory signaling, cellular immune responses and cardiac injury, which may outweigh their contribution to viral elimination.

importance of such subsets for disease development ${ }^{23}$. Also, gonadectomy in male mice protects them against CVB3 induced myocarditis by increasing the numbers of $M 2$ macrophages, Th2 cells, and T regulatory cells ${ }^{50}$. It is therefore likely that gender differences in susceptibility are governed by an intricate crosstalk between innate and adaptive mechanisms rather than a single culprit mechanism.

\section{Concluding remarks}

Management of viral myocarditis represents a difficult challenge for both clinicians and biomedical researchers. Factors including symptomatic and etiological heterogeneity, low incidence in the general population, and the limited collection of endomyocardial biopsies have complicated systematic investigation and the development of specific therapies. Because virulence and immune responses both contribute to the pathogenesis of viral myocarditis, both could be targets of therapeutic intervention. However, the clinical benefits of antiviral therapy to prevent an inflammatory response may be limited because most patients present in the subacute or chronic phases of disease. Broad suppression 
of inflammation has not shown benefits for patients with an aggregate, non-specified diagnosis of myocarditis ${ }^{17,25,121}$, however, there is evolving evidence that tailored treatment for patients subgroups may be feasible ${ }^{26,122-125}$. These insights are paralleled by a rapidly growing understanding of the inflammatory contribution to cardiac injury in viral myocarditis, which suggests that effective therapies should aim for selective intervention in the immune response rather than broad immunosuppression. In this review, we have summarized the available evidence for beneficial and detrimental pathways of both the innate and adaptive immune system during viral myocarditis. Such evidence suggests that potential targets for immune modulation include selectively stimulating or suppressing TLR signaling pathways (Figure 3), exogenous polarization of macrophage activation subtypes, and suppression of Th1 versus Th2 responses, for instance by stimulating regulatory $T$ cell differentiation (Figure 4).

The most clinically advanced strategy encompasses immune modulation through IFN- $\beta$ administration, which was suggested to benefit patients from the results of a 2003 phase II trial but is awaiting validation under randomized and placebo-controlled conditions $^{17,26}$. Finally, it will be important to extend the findings generated in experimental models of enteroviral myocarditis to models of other viruses such as PVB19 and HHV6, which are increasingly found in hearts of myocarditis patients and may differ in their response to treatment. While viral myocarditis remains a daunting task for cooperation between cardiologists and immunologists, steadily accumulating insights have identified novel therapeutic opportunities, such as modulating TLR activity and stimulating antiinflammatory cell types, that mandate further exploration and clinical translation in the near future.

\section{Acknowledgements}

We are grateful to Dr. Anna Papageorgiou for critical reading of the manuscript and acknowledge the contribution of Prof. Dr. Sally Huber for useful discussions. We thank Dr. Bart de Vries for providing immunohistological stainings of viral myocarditis and Wouter Verhesen for help with graphical illustrations. 


\section{References}

1. Cooper LT, Jr. Myocarditis. N Engl J Med. 2009;360:1526-1538

2. Sagar S, Liu PP, Cooper LT, Jr. Myocarditis. Lancet. 2011

3. Hahn EA, Hartz VL, Moon TE, O'Connell JB, Herskowitz A, McManus BM, Mason JW. The myocarditis treatment trial: Design, methods and patients enrollment. European heart journal. 1995;16 Suppl 0:162-167

4. Esfandiarei M, McManus BM. Molecular biology and pathogenesis of viral myocarditis. Annual review of pathology. 2008;3:127-155

5. Blauwet LA, Cooper LT. Myocarditis. Progress in cardiovascular diseases. 2010;52:274-288

6. McCarthy RE, 3rd, Boehmer JP, Hruban RH, Hutchins GM, Kasper EK, Hare JM, Baughman KL. Long-term outcome of fulminant myocarditis as compared with acute (nonfulminant) myocarditis. The New England journal of medicine. 2000;342:690-695

7. Dennert R, Crijns HJ, Heymans S. Acute viral myocarditis. Eur Heart J. 2008

8. Kuhl U, Pauschinger M, Noutsias M, Seeberg B, Bock T, Lassner D, Poller W, Kandolf R, Schultheiss HP. High prevalence of viral genomes and multiple viral infections in the myocardium of adults with "idiopathic" left ventricular dysfunction. Circulation. 2005;111:887-893

9. Kindermann I, Kindermann M, Kandolf R, Klingel K, Bultmann B, Muller T, Lindinger A, Bohm M. Predictors of outcome in patients with suspected myocarditis. Circulation. 2008;118:639-648

10. Kuhl U, Pauschinger M, Seeberg B, Lassner D, Noutsias M, Poller W, Schultheiss HP. Viral persistence in the myocardium is associated with progressive cardiac dysfunction. Circulation. 2005;112:1965-1970

11. Kim KS, Tracy S, Tapprich W, Bailey J, Lee CK, Kim K, Barry WH, Chapman NM. 5'-terminal deletions occur in coxsackievirus b3 during replication in murine hearts and cardiac myocyte cultures and correlate with encapsidation of negative-strand viral rna. Journal of virology. 2005;79:7024-7041

12. Klingel K, Hohenadl C, Canu A, Albrecht M, Seemann M, Mall G, Kandolf R. Ongoing enterovirus-induced myocarditis is associated with persistent heart muscle infection: Quantitative analysis of virus replication, tissue damage, and inflammation. Proceedings of the National Academy of Sciences of the United States of America. 1992;89:314-318

13. Wolff PG, Kuhl U, Schultheiss HP. Laminin distribution and autoantibodies to laminin in dilated cardiomyopathy and myocarditis. American heart journal. 1989;117:1303-1309

14. De Scheerder IK, De Buyzere M, Delanghe J, Maas A, Clement DL, Wieme R. Humoral immune response against contractile proteins (actin and myosin) during cardiovascular disease. European heart journal. 1991;12 Suppl D:88-94

15. Caforio AL, Goldman JH, Haven AJ, Baig KM, Libera LD, McKenna WJ. Circulating cardiac-specific autoantibodies as markers of autoimmunity in clinical and biopsy-proven myocarditis. The myocarditis treatment trial investigators. European heart journal. 1997;18:270-275

16. Schwimmbeck PL, Badorff C, Rohn G, Schulze K, Schultheiss HP. The role of sensitized t-cells in myocarditis and dilated cardiomyopathy. International journal of cardiology. 1996;54:117-125

17. Schultheiss HP, Kuhl U, Cooper LT. The management of myocarditis. European heart journal. 2011;32:26162625

18. Bock CT, Klingel K, Kandolf R. Human parvovirus b19-associated myocarditis. The New England journal of medicine. 2010;362:1248-1249

19. Badorff C, Lee GH, Lamphear BJ, Martone ME, Campbell KP, Rhoads RE, Knowlton KU. Enteroviral protease 2a cleaves dystrophin: Evidence of cytoskeletal disruption in an acquired cardiomyopathy. Nature medicine. 1999;5:320-326

20. Chow LH, Beisel KW, McManus BM. Enteroviral infection of mice with severe combined immunodeficiency. Evidence for direct viral pathogenesis of myocardial injury. Laboratory investigation; a journal of technical methods and pathology. 1992;66:24-31 
21. Woodruff JF, Woodruff JJ. Involvement of t lymphocytes in the pathogenesis of coxsackie virus b3 heart disease. Journal of immunology. 1974;113:1726-1734

22. Shi Y, Fukuoka M, Li G, Liu Y, Chen M, Konviser M, Chen X, Opavsky MA, Liu PP. Regulatory t cells protect mice against coxsackievirus-induced myocarditis through the transforming growth factor beta-coxsackieadenovirus receptor pathway. Circulation. 2010;121:2624-2634

23. Li K, Xu W, Guo Q, Jiang Z, Wang P, Yue Y, Xiong S. Differential macrophage polarization in male and female balb/c mice infected with coxsackievirus b3 defines susceptibility to viral myocarditis. Circulation research. 2009; 105:353-364

24. Heymans S, Hirsch E, Anker SD, Aukrust P, Balligand JL, Cohen-Tervaert JW, Drexler H, Filippatos G, Felix SB, Gullestad L, Hilfiker-Kleiner D, Janssens S, Latini R, Neubauer G, Paulus WJ, Pieske B, Ponikowski P, Schroen B, Schultheiss HP, Tschope C, Van Bilsen M, Zannad F, McMurray J, Shah AM. Inflammation as a therapeutic target in heart failure? A scientific statement from the translational research committee of the heart failure association of the european society of cardiology. Eur J Heart Fail. 2009;11:119-129

25. Mason JW, O'Connell JB, Herskowitz A, Rose NR, McManus BM, Billingham ME, Moon TE. A clinical trial of immunosuppressive therapy for myocarditis. The myocarditis treatment trial investigators. The New England journal of medicine. 1995;333:269-275

26. Kuhl U, Pauschinger M, Schwimmbeck PL, Seeberg B, Lober C, Noutsias M, Poller W, Schultheiss HP. Interferon-beta treatment eliminates cardiotropic viruses and improves left ventricular function in patients with myocardial persistence of viral genomes and left ventricular dysfunction. Circulation. 2003;107:2793-2798

27. Fuse K, Chan G, Liu Y, Gudgeon P, Husain M, Chen M, Yeh WC, Akira S, Liu PP. Myeloid differentiation factor-88 plays a crucial role in the pathogenesis of coxsackievirus b3-induced myocarditis and influences type i interferon production. Circulation. 2005;112:2276-2285

28. Riad A, Westermann D, Zietsch C, Savvatis K, Becher PM, Bereswill S, Heimesaat MM, Lettau O, Lassner D, Dorner A, Poller W, Busch M, Felix SB, Schultheiss HP, Tschope C. Trif is a critical survival factor in viral cardiomyopathy. Journal of immunology. 2011;186:2561-2570

29. Tomko RP, Xu R, Philipson L. Hcar and mcar: The human and mouse cellular receptors for subgroup c adenoviruses and group b coxsackieviruses. Proceedings of the National Academy of Sciences of the United States of America. 1997;94:3352-3356

30. Bergelson JM, Krithivas A, Celi L, Droguett G, Horwitz MS, Wickham T, Crowell RL, Finberg RW. The murine car homolog is a receptor for coxsackie b viruses and adenoviruses. Journal of virology. 1998;72:415-419

31. Kim KS, Hufnagel G, Chapman NM, Tracy S. The group b coxsackieviruses and myocarditis. Reviews in medical virology. 2001;11:355-368

32. Klingel K, Stephan S, Sauter M, Zell R, McManus BM, Bultmann B, Kandolf R. Pathogenesis of murine enterovirus myocarditis: Virus dissemination and immune cell targets. Journal of virology. 1996;70:88888895

33. Anderson DR, Wilson JE, Carthy CM, Yang D, Kandolf R, McManus BM. Direct interactions of coxsackievirus b3 with immune cells in the splenic compartment of mice susceptible or resistant to myocarditis. Journal of virology. 1996;70:4632-4645

34. Shi Y, Chen C, Lisewski U, Wrackmeyer U, Radke M, Westermann D, Sauter M, Tschope C, Poller W, Klingel K, Gotthardt M. Cardiac deletion of the coxsackievirus-adenovirus receptor abolishes coxsackievirus b3 infection and prevents myocarditis in vivo. Journal of the American College of Cardiology. 2009;53:1219-1226

35. Mann DL. The emerging role of innate immunity in the heart and vascular system: For whom the cell tolls. Circulation research. 2011;108:1133-1145

36. Seko Y, Takahashi N, Yagita H, Okumura K, Yazaki Y. Expression of cytokine mrnas in murine hearts with acute myocarditis caused by coxsackievirus b3. The Journal of pathology. 1997;183:105-108

37. Takaoka A, Hayakawa S, Yanai H, Stoiber D, Negishi H, Kikuchi H, Sasaki S, Imai K, Shibue T, Honda K, Taniguchi T. Integration of interferon-alpha/beta signalling to p53 responses in tumour suppression and antiviral defence. Nature. 2003;424:516-523

38. Papageorgiou AP, Heymans S. Interactions between the extracellular matrix and inflammation during viral myocarditis. Immunobiology. 2011 
39. Godeny EK, Gauntt CJ. Involvement of natural killer cells in coxsackievirus b3-induced murine myocarditis. Journal of immunology. 1986;137:1695-1702

40. Seko Y, Matsuda H, Kato K, Hashimoto Y, Yagita H, Okumura K, Yazaki Y. Expression of intercellular adhesion molecule-1 in murine hearts with acute myocarditis caused by coxsackievirus b3. The Journal of clinical investigation. 1993;91:1327-1336

41. Cho CT, Feng KK, McCarthy VP, Lenahan MF. Role of antiviral antibodies in resistance against coxsackievirus b3 infection: Interaction between preexisting antibodies and an interferon inducer. Infection and immunity. 1982;37:720-727

42. Chow LH, Gauntt CJ, McManus BM. Differential effects of myocarditic variants of coxsackievirus b3 in inbred mice. A pathologic characterization of heart tissue damage. Laboratory investigation; a journal of technical methods and pathology. 1991;64:55-64

43. Knowlton KU, Badorff $\mathrm{C}$. The immune system in viral myocarditis: Maintaining the balance. Circulation research. 1999;85:559-561

44. Huber SA, Stone JE, Wagner DH, Jr., Kupperman J, Pfeiffer L, David C, O'Brien RL, Davis GS, Newell MK. Gamma delta+ $t$ cells regulate major histocompatibility complex class ii(ia and ie)-dependent susceptibility to coxsackievirus b3-induced autoimmune myocarditis. Journal of virology. 1999;73:5630-5636

45. Huber SA, Pfaeffle B. Differential th1 and th2 cell responses in male and female balb/c mice infected with coxsackievirus group b type 3. Journal of virology. 1994;68:5126-5132

46. Rose NR. Myocarditis: Infection versus autoimmunity. Journal of clinical immunology. 2009;29:730-737

47. Weinzierl AO, Szalay G, Wolburg H, Sauter M, Rammensee HG, Kandolf R, Stevanovic S, Klingel K. Effective chemokine secretion by dendritic cells and expansion of cross-presenting cd4-/cd8+ dendritic cells define a protective phenotype in the mouse model of coxsackievirus myocarditis. Journal of virology. 2008;82:81498160

48. Jakel S, Kuckelkorn U, Szalay G, Plotz M, Textoris-Taube K, Opitz E, Klingel K, Stevanovic S, Kandolf R, Kotsch K, Stangl K, Kloetzel PM, Voigt A. Differential interferon responses enhance viral epitope generation by myocardial immunoproteasomes in murine enterovirus myocarditis. The American journal of pathology. 2009;175:510-518

49. Yajima T. Viral myocarditis: Potential defense mechanisms within the cardiomyocyte against virus infection. Future microbiology. 2011;6:551-566

50. Frisancho-Kiss S, Coronado MJ, Frisancho JA, Lau VM, Rose NR, Klein SL, Fairweather D. Gonadectomy of male balb/c mice increases tim-3(+) alternatively activated $\mathrm{m} 2$ macrophages, tim-3(+) t cells, th2 cells and treg in the heart during acute coxsackievirus-induced myocarditis. Brain, behavior, and immunity. 2009;23:649657

51. Lee C, Maull E, Chapman N, Tracy S, Wood J, Gauntt C. Generation of an infectious cdna of a highly cardiovirulent coxsackievirus b3(cvb3m) and comparison to other infectious cvb3 cdnas. Virus research. $1997 ; 50: 225-235$

52. Kandolf $\mathrm{R}$, Hofschneider PH. Molecular cloning of the genome of a cardiotropic coxsackie b3 virus: Fulllength reverse-transcribed recombinant cdna generates infectious virus in mammalian cells. Proceedings of the National Academy of Sciences of the United States of America. 1985;82:4818-4822

53. Woodruff JF. Viral myocarditis. A review. The American journal of pathology. 1980;101:425-484

54. Frisancho-Kiss S, Nyland JF, Davis SE, Frisancho JA, Barrett MA, Rose NR, Fairweather D. Sex differences in coxsackievirus b3-induced myocarditis: II-12rbeta1 signaling and ifn-gamma increase inflammation in males independent from stat4. Brain research. 2006;1126:139-147

55. Kawai C. From myocarditis to cardiomyopathy: Mechanisms of inflammation and cell death: Learning from the past for the future. Circulation. 1999;99:1091-1100

56. Yajima T, Knowlton KU. Viral myocarditis: From the perspective of the virus. Circulation. 2009;119:2615-2624

57. McManus BM, Chow LH, Wilson JE, Anderson DR, Gulizia JM, Gauntt CJ, Klingel KE, Beisel KW, Kandolf R. Direct myocardial injury by enterovirus: A central role in the evolution of murine myocarditis. Clinical immunology and immunopathology. 1993;68:159-169 
58. Wessely R, Klingel K, Santana LF, Dalton N, Hongo M, Jonathan Lederer W, Kandolf R, Knowlton KU. Transgenic expression of replication-restricted enteroviral genomes in heart muscle induces defective excitationcontraction coupling and dilated cardiomyopathy. The Journal of clinical investigation. 1998;102:1444-1453

59. Badorff C, Knowlton KU. Dystrophin disruption in enterovirus-induced myocarditis and dilated cardiomyopathy: From bench to bedside. Medical microbiology and immunology. 2004;193:121-126

60. Xiong D, Yajima T, Lim BK, Stenbit A, Dublin A, Dalton ND, Summers-Torres D, Molkentin JD, Duplain H, Wessely R, Chen J, Knowlton KU. Inducible cardiac-restricted expression of enteroviral protease 2a is sufficient to induce dilated cardiomyopathy. Circulation. 2007;115:94-102

61. Fairweather D, Frisancho-Kiss S, Njoku DB, Nyland JF, Kaya Z, Yusung SA, Davis SE, Frisancho JA, Barrett MA, Rose NR. Complement receptor 1 and 2 deficiency increases coxsackievirus b3-induced myocarditis, dilated cardiomyopathy, and heart failure by increasing macrophages, il-1beta, and immune complex deposition in the heart. Journal of immunology. 2006;176:3516-3524

62. Anderson DR, Carthy CM, Wilson JE, Yang D, Devine DV, McManus BM. Complement component 3 interactions with coxsackievirus b3 capsid proteins: Innate immunity and the rapid formation of splenic antiviral germinal centers. Journal of virology. 1997;71:8841-8845

63. Godeny EK, Gauntt CJ. Murine natural killer cells limit coxsackievirus b3 replication. Journal of immunology. 1987; 139:913-918

64. Wu CY, Feng Y, Qian GC, Wu JH, Luo J, Wang Y, Chen GJ, Guo XK, Wang ZJ. Alpha-galactosylceramide protects mice from lethal coxsackievirus b3 infection and subsequent myocarditis. Clinical and experimental immunology. 2010;162:178-187

65. Hirasawa K, Tsutsui S, Takeda M, Mizutani M, Itagaki S, Doi K. Depletion of mac1-positive macrophages protects dba/2 mice from encephalomyocarditis virus-induced myocarditis and diabetes. The Journal of general virology. 1996;77 (Pt 4):737-741

66. Gordon S, Martinez FO. Alternative activation of macrophages: Mechanism and functions. Immunity. 2010;32:593-604

67. Lowenstein CJ, Hill SL, Lafond-Walker A, Wu J, Allen G, Landavere M, Rose NR, Herskowitz A. Nitric oxide inhibits viral replication in murine myocarditis. The Journal of clinical investigation. 1996;97:1837-1843

68. Zaragoza C, Ocampo C, Saura M, Leppo M, Wei XQ, Quick R, Moncada S, Liew FY, Lowenstein CJ. The role of inducible nitric oxide synthase in the host response to coxsackievirus myocarditis. Proceedings of the National Academy of Sciences of the United States of America. 1998;95:2469-2474

69. Lee JK, Zaidi SH, Liu P, Dawood F, Cheah AY, Wen WH, Saiki Y, Rabinovitch M. A serine elastase inhibitor reduces inflammation and fibrosis and preserves cardiac function after experimentally-induced murine myocarditis. Nature medicine. 1998;4:1383-1391

70. Negishi H, Osawa T, Ogami K, Ouyang X, Sakaguchi S, Koshiba R, Yanai H, Seko Y, Shitara H, Bishop K, Yonekawa H, Tamura T, Kaisho T, Taya C, Taniguchi T, Honda K. A critical link between toll-like receptor 3 and type ii interferon signaling pathways in antiviral innate immunity. Proceedings of the National Academy of Sciences of the United States of America. 2008;105:20446-20451

71. Fairweather D, Yusung S, Frisancho S, Barrett M, Gatewood S, Steele R, Rose NR. II-12 receptor beta 1 and toll-like receptor 4 increase il-1 beta- and il-18-associated myocarditis and coxsackievirus replication. Journal of immunology. 2003;170:4731-4737

72. Riad A, Westermann D, Escher F, Becher PM, Savvatis K, Lettau O, Heimesaat MM, Bereswill S, Volk HD, Schultheiss HP, Tschope C. Myeloid differentiation factor-88 contributes to tlr9-mediated modulation of acute coxsackievirus b3-induced myocarditis in vivo. American journal of physiology. Heart and circulatory physiology. 2010;298:H2024-2031

73. Lutton CW, Gauntt CJ. Ameliorating effect of ifn-beta and anti-ifn-beta on coxsackievirus b3-induced myocarditis in mice. Journal of interferon research. 1985;5:137-146

74. Deonarain R, Cerullo D, Fuse K, Liu PP, Fish EN. Protective role for interferon-beta in coxsackievirus b3 infection. Circulation. 2004;110:3540-3543

75. Wessely R, Klingel K, Knowlton KU, Kandolf R. Cardioselective infection with coxsackievirus b3 requires intact type i interferon signaling: Implications for mortality and early viral replication. Circulation. 2001;103:756-761 
76. Horwitz MS, La Cava A, Fine C, Rodriguez E, llic A, Sarvetnick N. Pancreatic expression of interferongamma protects mice from lethal coxsackievirus b3 infection and subsequent myocarditis. Nature medicine. 2000;6:693-697

77. Yasukawa H, Yajima T, Duplain H, Iwatate M, Kido M, Hoshijima M, Weitzman MD, Nakamura T, Woodard S, Xiong D, Yoshimura A, Chien KR, Knowlton KU. The suppressor of cytokine signaling-1 (socs1) is a novel therapeutic target for enterovirus-induced cardiac injury. The Journal of clinical investigation. 2003;111:469478

78. Yajima T, Yasukawa H, Jeon ES, Xiong D, Dorner A, Iwatate M, Nara M, Zhou H, Summers-Torres D, Hoshijima M, Chien KR, Yoshimura A, Knowlton KU. Innate defense mechanism against virus infection within the cardiac myocyte requiring gp130-stat3 signaling. Circulation. 2006;114:2364-2373

79. Lane JR, Neumann DA, Lafond-Walker A, Herskowitz A, Rose NR. Interleukin 1 or tumor necrosis factor can promote coxsackie b3-induced myocarditis in resistant b10.A mice. The Journal of experimental medicine. 1992;175:1123-1129

80. Huang $\mathrm{CH}$, Vallejo JG, Kollias G, Mann DL. Role of the innate immune system in acute viral myocarditis. Basic research in cardiology. 2009;104:228-237

81. Wada H, Saito K, Kanda T, Kobayashi I, Fujii H, Fujigaki S, Maekawa N, Takatsu H, Fujiwara H, Sekikawa K, Seishima M. Tumor necrosis factor-alpha (tnf-alpha) plays a protective role in acute viralmyocarditis in mice: A study using mice lacking tnf-alpha. Circulation. 2001;103:743-749

82. Tanaka T, Kanda T, McManus BM, Kanai H, Akiyama H, Sekiguchi K, Yokoyama T, Kurabayashi M. Overexpression of interleukin-6 aggravates viral myocarditis: Impaired increase in tumor necrosis factor-alpha. Journal of molecular and cellular cardiology. 2001;33:1627-1635

83. Kanda T, McManus JE, Nagai R, Imai S, Suzuki T, Yang D, McManus BM, Kobayashi I. Modification of viral myocarditis in mice by interleukin-6. Circulation research. 1996;78:848-856

84. Kanda T, Tanaka T, Sekiguchi K, Seta Y, Kurimoto M, Wilson McManus JE, Nagai R, Yang D, McManus BM, Kobayashi I. Effect of interleukin-18 on viral myocarditis: Enhancement of interferon- gamma and natural killer cell activity. Journal of molecular and cellular cardiology. 2000;32:2163-2171

85. Cook DN, Beck MA, Coffman TM, Kirby SL, Sheridan JF, Pragnell IB, Smithies O. Requirement of mip-1 alpha for an inflammatory response to viral infection. Science. 1995;269:1583-1585

86. Yue Y, Gui J, Ai W, Xu W, Xiong S. Direct gene transfer with ip-10 mutant ameliorates mouse cvb3-induced myocarditis by blunting th1 immune responses. PLoS ONE. 2011;6:e18186

87. Szalay G, Sauter M, Hald J, Weinzierl A, Kandolf R, Klingel K. Sustained nitric oxide synthesis contributes to immunopathology in ongoing myocarditis attributable to interleukin-10 disorders. The American journal of pathology. 2006;169:2085-2093

88. Horwitz MS, Knudsen M, Ilic A, Fine C, Sarvetnick N. Transforming growth factor-beta inhibits coxsackievirusmediated autoimmune myocarditis. Viral immunology. 2006;19:722-733

89. Frisancho-Kiss S, Nyland JF, Davis SE, Barrett MA, Gatewood SJ, Njoku DB, Cihakova D, Silbergeld EK, Rose NR, Fairweather D. Cutting edge: T cell ig mucin-3 reduces inflammatory heart disease by increasing ctla-4 during innate immunity. Journal of immunology. 2006;176:6411-6415

90. Heymans S, Pauschinger M, De Palma A, Kallwellis-Opara A, Rutschow S, Swinnen M, Vanhoutte D, Gao F, Torpai R, Baker AH, Padalko E, Neyts J, Schultheiss HP, Van de Werf F, Carmeliet P, Pinto YM. Inhibition of urokinase-type plasminogen activator or matrix metalloproteinases prevents cardiac injury and dysfunction during viral myocarditis. Circulation. 2006;114:565-573

91. Cheung C, Marchant D, Walker EK, Luo Z, Zhang J, Yanagawa B, Rahmani M, Cox J, Overall C, Senior RM, Luo H, McManus BM. Ablation of matrix metalloproteinase-9 increases severity of viral myocarditis in mice. Circulation. 2008;117:1574-1582

92. Westermann D, Savvatis K, Lindner D, Zietsch C, Becher PM, Hammer E, Heimesaat MM, Bereswill S, Volker U, Escher F, Riad A, Plendl J, Klingel K, Poller W, Schultheiss HP, Tschope C. Reduced degradation of the chemokine mcp-3 by matrix metalloproteinase-2 exacerbates myocardial inflammation in experimental viral cardiomyopathy. Circulation. 2011;124:2082-2093

93. Huber SA. Autoimmunity in coxsackievirus b3 induced myocarditis. Autoimmunity. 2006;39:55-61 
94. Latif N, Zhang H, Archard LC, Yacoub MH, Dunn MJ. Characterization of anti-heart antibodies in mice after infection with coxsackie b3 virus. Clinical immunology. 1999;91:90-98

95. Caforio AL, Mahon NJ, McKenna WJ. Cardiac autoantibodies to myosin and other heart-specific autoantigens in myocarditis and dilated cardiomyopathy. Autoimmunity. 2001;34:199-204

96. Gauntt CJ, Arizpe HM, Higdon AL, Wood HJ, Bowers DF, Rozek MM, Crawley R. Molecular mimicry, anticoxsackievirus b3 neutralizing monoclonal antibodies, and myocarditis. Journal of immunology. 1995;154:29832995

97. Eriksson U, Ricci R, Hunziker L, Kurrer MO, Oudit GY, Watts TH, Sonderegger I, Bachmaier K, Kopf M, Penninger JM. Dendritic cell-induced autoimmune heart failure requires cooperation between adaptive and innate immunity. Nature medicine. 2003;9:1484-1490

98. Eriksson U, Kurrer MO, Sonderegger I, lezzi G, Tafuri A, Hunziker L, Suzuki S, Bachmaier K, Bingisser RM, Penninger JM, Kopf M. Activation of dendritic cells through the interleukin 1 receptor 1 is critical for the induction of autoimmune myocarditis. The Journal of experimental medicine. 2003;197:323-331

99. Hashimoto I, Komatsu T. Myocardial changes after infection with coxsackie virus b3 in nude mice. British journal of experimental pathology. 1978;59:13-20

100. Robinson JA, O'Connell JB, Roeges LM, Major EO, Gunnar RM. Coxsackie b3 myocarditis in athymic mice. Proceedings of the Society for Experimental Biology and Medicine. Society for Experimental Biology and Medicine. 1981;166:80-91

101. Schnurr DP, Schmidt NJ. Coxsackievirus b3 persistence and myocarditis in nfr nu/nu and $+/$ nu mice. Medical microbiology and immunology. 1984;173:1-7

102. Opavsky MA, Penninger J, Aitken K, Wen WH, Dawood F, Mak T, Liu P. Susceptibility to myocarditis is dependent on the response of alphabeta $t$ lymphocytes to coxsackieviral infection. Circulation research. 1999;85:551-558

103. Klingel K, Schnorr JJ, Sauter M, Szalay G, Kandolf R. Beta2-microglobulin-associated regulation of interferongamma and virus-specific immunoglobulin $\mathrm{g}$ confer resistance against the development of chronic coxsackievirus myocarditis. The American journal of pathology. 2003;162:1709-1720

104. Gebhard JR, Perry CM, Harkins S, Lane T, Mena I, Asensio VC, Campbell IL, Whitton JL. Coxsackievirus b3-induced myocarditis: Perforin exacerbates disease, but plays no detectable role in virus clearance. The American journal of pathology. 1998;153:417-428

105. Huber SA. T cells expressing the gamma delta $t$ cell receptor induce apoptosis in cardiac myocytes. Cardiovascular research. 2000;45:579-587

106. Huber S, Shi C, Budd RC. Gammadelta t cells promote a th1 response during coxsackievirus b3 infection in vivo: Role of fas and fas ligand. Journal of virology. 2002;76:6487-6494

107. Huber SA, Kupperman J, Newell MK. Hormonal regulation of $\mathrm{cd} 4(+)$ t-cell responses in coxsackievirus b3induced myocarditis in mice. Journal of virology. 1999;73:4689-4695

108. Wing K, Sakaguchi S. Regulatory t cells exert checks and balances on self tolerance and autoimmunity. Nature immunology. 2010;11:7-13

109. Huber SA, Feldman AM, Sartini D. Coxsackievirus b3 induces t regulatory cells, which inhibit cardiomyopathy in tumor necrosis factor-alpha transgenic mice. Circulation research. 2006;99:1109-1116

110. Leipner C, Borchers M, Merkle I, Stelzner A. Coxsackievirus b3-induced myocarditis in mhc class ii-deficient mice. Journal of human virology. 1999;2:102-114

111. Mena I, Perry CM, Harkins S, Rodriguez F, Gebhard J, Whitton JL. The role of b lymphocytes in coxsackievirus b3 infection. The American journal of pathology. 1999;155:1205-1215

112. Marchant DJ, McManus BM. Regulating viral myocarditis: Allografted regulatory $t$ cells decrease immune infiltration and viral load. Circulation. 2010;121:2609-2611

113. Marchant D, Dou Y, Luo H, Garmaroudi FS, McDonough JE, Si X, Walker E, Luo Z, Arner A, Hegele RG, Laher I, McManus BM. Bosentan enhances viral load via endothelin-1 receptor type-a-mediated p38 mitogenactivated protein kinase activation while improving cardiac function during coxsackievirus-induced myocarditis. Circulation research. 2009;104:813-821 
114. Esfandiarei M, Suarez A, Amaral A, Si X, Rahmani M, Dedhar S, McManus BM. Novel role for integrin-linked kinase in modulation of coxsackievirus b3 replication and virus-induced cardiomyocyte injury. Circulation research. 2006;99:354-361

115. Marchant D, Si X, Luo H, McManus B, Yang D. The impact of cvb3 infection on host cell biology. Current topics in microbiology and immunology. 2008;323:177-198

116. Si X, Luo H, Morgan A, Zhang J, Wong J, Yuan J, Esfandiarei M, Gao G, Cheung C, McManus BM. Stressactivated protein kinases are involved in coxsackievirus b3 viral progeny release. Journal of virology. 2005;79:13875-13881

117. Han J, Lee JD, Bibbs L, Ulevitch RJ. A map kinase targeted by endotoxin and hyperosmolarity in mammalian cells. Science. 1994;265:808-811

118. Tudor C, Marchese FP, Hitti E, Aubareda A, Rawlinson L, Gaestel M, Blackshear PJ, Clark AR, Saklatvala J, Dean JL. The p38 mapk pathway inhibits tristetraprolin-directed decay of interleukin-10 and pro-inflammatory mediator mrnas in murine macrophages. FEBS letters. 2009;583:1933-1938

119. Liu P, Aitken K, Kong YY, Opavsky MA, Martino T, Dawood F, Wen WH, Kozieradzki I, Bachmaier K, Straus D, Mak TW, Penninger JM. The tyrosine kinase p56lck is essential in coxsackievirus b3-mediated heart disease. Nature medicine. 2000;6:429-434

120. Frisancho-Kiss S, Davis SE, Nyland JF, Frisancho JA, Cihakova D, Barrett MA, Rose NR, Fairweather D. Cutting edge: Cross-regulation by tlr4 and t cell ig mucin-3 determines sex differences in inflammatory heart disease. Journal of immunology. 2007;178:6710-6714

121. McNamara DM, Holubkov R, Starling RC, Dec GW, Loh E, Torre-Amione G, Gass A, Janosko K, Tokarczyk T, Kessler P, Mann DL, Feldman AM. Controlled trial of intravenous immune globulin in recent-onset dilated cardiomyopathy. Circulation. 2001;103:2254-2259

122. Frustaci A, Chimenti C, Calabrese F, Pieroni M, Thiene G, Maseri A. Immunosuppressive therapy for active lymphocytic myocarditis: Virological and immunologic profile of responders versus nonresponders. Circulation. 2003; 107:857-863

123. Frustaci A, Russo MA, Chimenti C. Randomized study on the efficacy of immunosuppressive therapy in patients with virus-negative inflammatory cardiomyopathy: The timic study. European heart journal. 2009;30:19952002

124. Parrillo JE, Cunnion RE, Epstein SE, Parker MM, Suffredini AF, Brenner M, Schaer GL, Palmeri ST, Cannon RO, 3rd, Alling D, et al. A prospective, randomized, controlled trial of prednisone for dilated cardiomyopathy. The New England journal of medicine. 1989;321:1061-1068

125. Wojnicz R, Nowalany-Kozielska E, Wojciechowska C, Glanowska G, Wilczewski P, Niklewski T, Zembala M, Polonski L, Rozek MM, Wodniecki J. Randomized, placebo-controlled study for immunosuppressive treatment of inflammatory dilated cardiomyopathy: Two-year follow-up results. Circulation. 2001;104:39-45

126. Huber S, Sartini D, Exley M. Role of cd1d in coxsackievirus b3-induced myocarditis. Journal of immunology. 2003; 170:3147-3153 


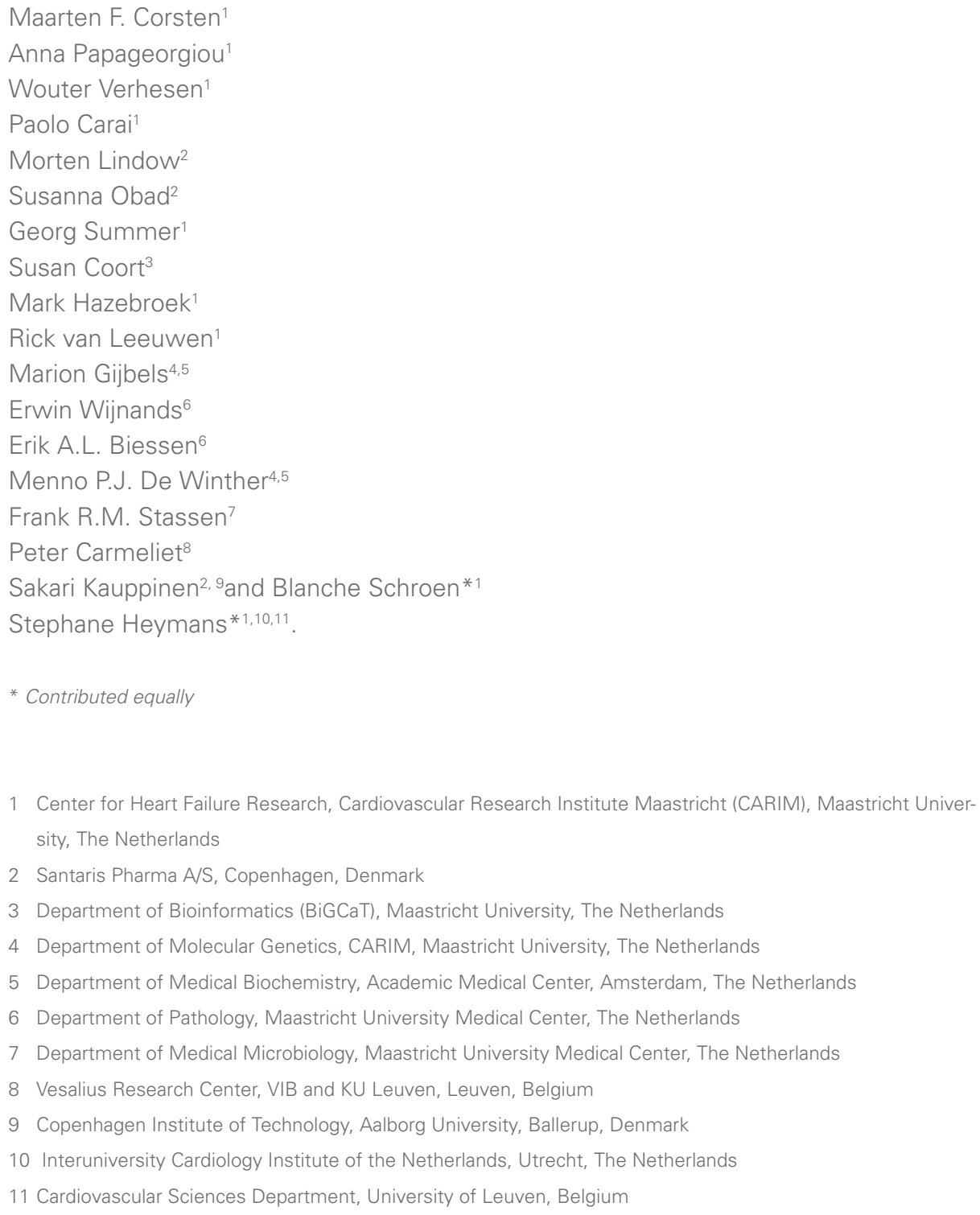

1 Center for Heart Failure Research, Cardiovascular Research Institute Maastricht (CARIM), Maastricht University, The Netherlands

2 Santaris Pharma A/S, Copenhagen, Denmark

3 Department of Bioinformatics (BiGCaT), Maastricht University, The Netherlands

4 Department of Molecular Genetics, CARIM, Maastricht University, The Netherlands

5 Department of Medical Biochemistry, Academic Medical Center, Amsterdam, The Netherlands

6 Department of Pathology, Maastricht University Medical Center, The Netherlands

7 Department of Medical Microbiology, Maastricht University Medical Center, The Netherlands

8 Vesalius Research Center, VIB and KU Leuven, Leuven, Belgium

9 Copenhagen Institute of Technology, Aalborg University, Ballerup, Denmark

10 Interuniversity Cardiology Institute of the Netherlands, Utrecht, The Netherlands

11 Cardiovascular Sciences Department, University of Leuven, Belgium 


\title{
1. MicroRNA profiling identifies microRNA-155 as an adverse mediator of cardiac injury and dysfunction during acute viral myocarditis
}

\author{
Modified from Circulation Research 2012;111:415-425 [IF 9.5]
}

\section{ABSTRACT}

Rationale viral myocarditis results from an adverse immune response to cardiotropic viruses, which causes irreversible myocyte destruction and heart failure in previously healthy people. The involvement of microRNAs and their usefulness as therapeutic targets in this process are unknown.

Objective To identify microRNAs involved in viral myocarditis pathogenesis and susceptibility.

Methods and Results Cardiac microRNAs were profiled in both human myocarditis and in Coxsackievirus B3-injected mice, comparing myocarditis-susceptible to non-susceptible mouse strains longitudinally. MicroRNA responses diverged depending on the susceptibility to myocarditis after viral infection in mice. MicroRNA-155, -146b and -21 were consistently and strongly up-regulated during acute myocarditis in both humans and susceptible mice. We found that microRNA-155 expression during myocarditis was localized primarily in infiltrating macrophages and T Iymphocytes. Inhibition of microRNA-155 by a systemically delivered LNA-antimiR attenuated cardiac infiltration by monocyte-macrophages, decreased T lymphocyte activation and reduced myocardial damage in acute myocarditis in mice. These changes were accompanied by the de-repression of the direct microRNA-155 target PU. 1 in cardiac inflammatory cells. Beyond the acute phase, microRNA-155 inhibition reduced mortality and improved cardiac function during 7 weeks of follow-up.

Conclusions our data show that cardiac microRNA dysregulation is a characteristic of both human and mouse viral myocarditis. The inflammatory microRNA-155 is up-regulated during acute myocarditis, contributes to the adverse inflammatory response to viral infection of the heart, and is a potential therapeutic target for viral myocarditis. 


\section{Introduction}

Acute viral myocarditis (VM) is an important cause of sudden cardiac death and heart failure in otherwise healthy young persons ${ }^{1}$. Its pathogenesis is based on an adverse immune response evoked by infection of the cardiac muscle by cardiotropic viruses, which leads to viral elimination as well as cardiac myocyte destruction, reparative fibrosis and heart failure. The lack of effective therapies to treat myocarditis mandates a better understanding of the basic molecular mechanisms that govern the adequate and autodestructive inflammatory signaling pathways within the immune system². Here, we investigated the hitherto unknown role of microRNAs as possible molecular drivers in the pathogenesis of VM.

MicroRNAs (miRNAs) represent a class of small non-coding RNAs that control the expression of entire networks of complementary transcripts ${ }^{3}$. In the heart, miRNAs have been shown to be necessary for normal embryonic development4 and homeostasis ${ }^{5}$. While individual miRNAs have been linked to specific cardiac diseases including hypertrophic

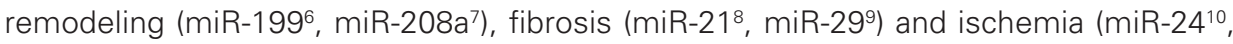
miR-499 ${ }^{11}$, miR-92a ${ }^{12}$ ), the role of miRNAs in inflammatory cardiac processes and more specifically during VM has not yet been established. Here, we report diverging expression profiles of miRNAs in VM-susceptible compared to VM-resistant mouse strains. Among others, miR-155, a known pro-inflammatory regulator of myeloid and lymphoid immune cell function ${ }^{13-15}$, is consistently up-regulated in human and mouse cardiac samples of VM during the acute inflammatory phase, and is primarily expressed by recruited inflammatory cells. Pharmacological inhibition of miR-155 by a systemically delivered locked nucleic acid (LNA)-antimiR - a class of miRNA inhibitors that has been successfully used in primate studies $^{16,17}$ and is currently in human clinical trials - attenuates cardiac inflammation and necrosis in acute mouse VM, and reduces mortality and contractile dysfunction in long-term follow-up. These data identify miR-155 as a contributor to the adverse inflammatory response and a potential therapeutic target in VM.

\section{Methods}

An expanded Methods section is available in the Supplemental material

\section{Animal studies}

All mouse experiments were performed according to the local relevant guidelines. For viral myocarditis (VM) miRNA profiling experiments, 4-week-old male susceptible $\mathrm{C} 3 \mathrm{H}$ mice or resistant N-strain C57BI/6 mice ${ }^{18}$ (Harlan, Boxmeer, The Netherlands) were used ( $\mathrm{n}=3$ per group). For microRNA knockdown in vivo, mice received 3 iv. injections per week $(25 \mathrm{mg} /$ kg/injection) of a 15-mer LNA-antimiR oligonucleotide targeting mmu-miR-155 or an LNA control. Chronic treatment for the 7-week-follow-up included 2 weeks of high dose treatment ( $25 \mathrm{mg} / \mathrm{kg} /$ injection iv) followed by 5 weeks of low dose treatment ( $5 \mathrm{mg} / \mathrm{kg} /$ injection ip). Group sizes were at least $n=6$ for sham and $n=11$ for VM groups in the 1-week 
analysis, $n=6$ per sham and $n=8$ for VM for flow cytometry, and $n=6$ for sham and $n=20$ for $\mathrm{VM}$ in the 7-week analysis.

\section{Patient material}

All human material was obtained during routine clinical sampling and available for research purposes in accordance with the Declaration of Helsinki and the ethical committee at Maastricht University Medical Center. MicroRNAs were profiled in right ventricular septal biopsies from patients with acute myocarditis $(n=4)$ with a definite clinical history of myocarditis ${ }^{1}$ and confirmed virus presence in the cardiac biopsies (Supplementary Table 1). Controls $(n=6)$ consisted of age-matched patients with unexplained ventricular tachyarrhythmias but with a normal ejection fraction and the absence of systemic or cardiac inflammation or virus presence at the time of biopsy (Supplementary Table 1).

\section{In situ hybridization}

In situ hybridization was performed as described previously ${ }^{19,20}$. Details are available the Methods section in the online-only Data Supplement.

\section{MicroRNA and Messenger RNA expression array analysis}

For microRNA array analysis, total RNA from human biopsies was hybridized to Illumina Human miRNAv2 Expression Panel arrays by ServiceXS (Leiden, The Netherlands) and total RNA from mouse left ventricles was hybridized to $\mu$ Paraflo ${ }^{\circledR}$ arrays (LC Sciences, Houston, TX). Detailed information about arrays analyses are available in the Methods section in the Data Supplement. Messenger RNA analysis from hearts of LNA control- and LNA-antimiR155-treated mice (sham and 7 days post CVB3 injection) was performed by hybridizing to Affymetrix GeneChip Mouse Gene 1.0 ST arrays.

\section{Statistical analyses}

Statistical analyses for miRNA arrays were performed in R. Analyses for experimental work were performed using GraphPad Prism 5.0d for Macintosh (GraphPad Software Inc., La Jolla, CA, USA). Pearson's correlation coefficients were determined to study correlations of quantitative variables in data sets with a Gaussian distribution. Comparisons between 2 groups were performed with two-tailed Student's t testing for Gaussian data or MannWhitney tests for non-Gaussian data. For comparisons of more than 2 groups, one-way ANOVA was used, followed by post hoc testing using Bonferroni correction for more groups. Probability values below 0.05 were considered statistically significant. Data are presented as mean \pm SEM unless otherwise indicated.

\section{Results}

Cardiac miRNA profiles during Coxsackievirus B3 induced murine viral myocarditis To identify miRNAs with a role in acute viral myocarditis (VM) we first analyzed miRNA expression profiles of Coxsackievirus B3 (CVB3)-induced VM in mice. To differentiate 
between miRNA changes associated with viral infection and inflammation, two mouse strains were used: $\mathrm{C} 3 \mathrm{H}$ mice that are highly susceptible to CVB3-induced myocarditis and C57BI/6N mice that are resistant to CVB3-induced cardiac inflammation despite similar accumulation of viral genomes in the heart ${ }^{18}$. Cardiac miRNA expression was evaluated at baseline, and days 4 and 7 after CVB3 inoculation to study viral replication before and after the onset of macroscopic inflammation, respectively. Histological analysis confirmed extensive myocyte necrosis and leukocyte infiltration in $\mathrm{C} 3 \mathrm{H}$ mice at 7 days, whereas only small patches of inflammation were observed in C57BI/6N animals (Figure 1A and B). Expression of CVB3 virus was detected in all mice at days 4 and 7, and did not significantly differ between strains (Figure 1C).

A heat map of cardiac miRNA expression during $V M$ in both strains shows diverging expression between the strains for clusters of miRNAs, most prominently after the onset of inflammation at day 7 after infection (Figure 1D-F; Supplementary Figure 1A and 2A).

a

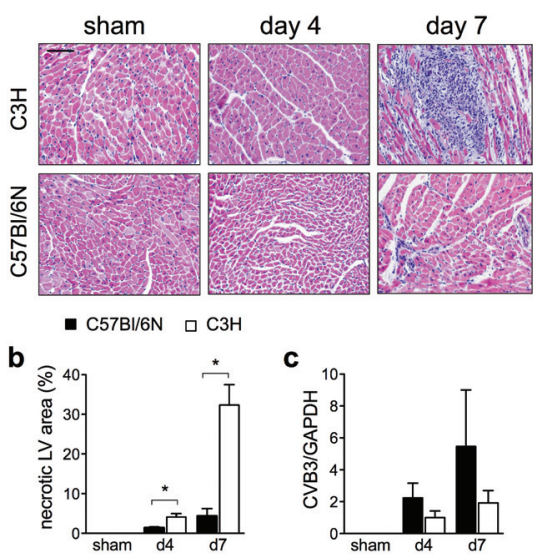

e

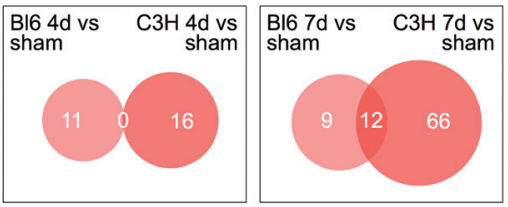

d

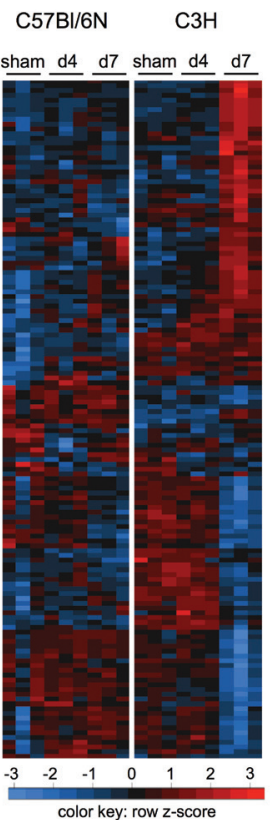

f
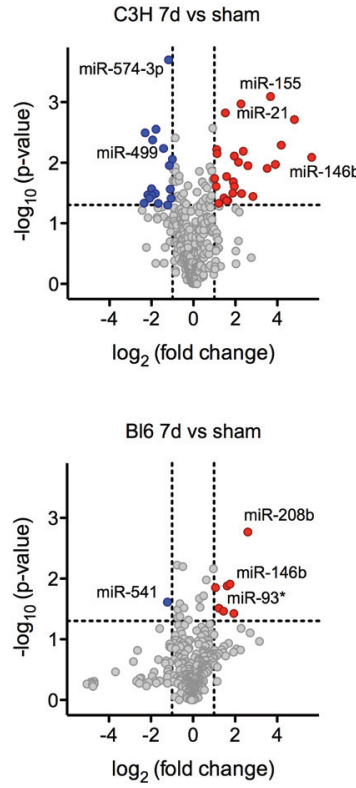

Figure 1. MicroRNA (miRNA) expression profiles of viral myocarditis in susceptible (C3H) or nonsusceptible (C57BI/6N) mice. (A, B) H\&E staining of left ventricular sections from $\mathrm{C} 3 \mathrm{H}$ and $\mathrm{C} 57 \mathrm{BI} / 6 \mathrm{~N}$ mice showing absence of necrosis in sham hearts and minimal necrosis at day 4 after CVB3 infection. Necrosis at day 7 was necrosis in $\mathrm{C} 57 \mathrm{BI} / 6 \mathrm{~N}$ animals $(4.4 \%$ of left ventricular area) but widespread in $\mathrm{C} 3 \mathrm{H}$ animals (32.3\% of left ventricular area) ( $n=6$ per group). (C) CVB3 viral genome expression did not significantly differ between mouse strains at day 4 or 7 and was undetectable in sham mice. (D) Heat map of differentially regulated miRNAs $(p<0.05)$ in $C 57 \mathrm{BI} / 6 \mathrm{~N}$ and $\mathrm{C} 3 \mathrm{H}$ mice. (E) Venn diagrams showing the numbers and overlap of differentially regulated miRNAs $(p<0.05)$ between groups. The most profound changes occur during myocarditis (day 7) in $\mathrm{C} 3 \mathrm{H}$ mice. (F) Volcano plots showing fold changes (log2 values) and $\mathrm{p}$ values $\left(\log _{10}\right)$ for individual miRNAs. ${ }^{*} \mathrm{P}<0.05$. Scale bar: $100 \mu \mathrm{m}$ 
The most extensive differences were observed in the highly inflamed $\mathrm{C} 3 \mathrm{H}$ hearts at 7 days compared to sham, where miRNA changes were markedly larger than in the mildly inflamed C57BI/6N hearts: 78 versus 221 miRNAs were differentially regulated at 7 days compared to sham in $\mathrm{C} 3 \mathrm{H}$ mice and $\mathrm{C} 57 \mathrm{BI} / 6 \mathrm{~N}$ mice, respectively. The majority of these differentially regulated miRNAs in $\mathrm{C} 57 \mathrm{BI} / 6 \mathrm{~N}$ animals overlapped with the $\mathrm{C} 3 \mathrm{H}$ miRNA changes, indicating consistency in the inflammatory response that was mild but not absent in C57BI/6N, as shown in Figure $1 \mathrm{~A}$ and $\mathrm{B}$.

At day 4 after infection, during cardiac viral replication but before infiltration of immune cells, 11 (resistant C57BI/6N) and 16 miRNAs (susceptible $\mathrm{C} 3 \mathrm{H}$ ) were differentially regulated as compared to sham. These miRNA changes showed little overlap with day 7 changes in both strains, indicating that day 4 differences reflect biological signaling distinct from inflammation (Supplementary Figure 1B). Importantly, the miRNAs differentially expressed at day 4 did not overlap between species, reflecting the diverging molecular response to CVB3 infection that may determine the severity of cardiac inflammation.

\section{Individual cardiac miRNAs are regulated during Coxsackievirus B3 induced murine viral myocarditis}

We next focused on individual dysregulated miRNAs. Notably, miRNAs that are well known for their roles in the immune system and inflammatory responses ${ }^{21}$ were among the most strongly up-regulated miRNAs during the inflammatory phase of $\mathrm{VM}(\mathrm{C} 3 \mathrm{H}$ mice at day 7), including miR-155, miR-21, miR-146a, miR-146b, and miR-223 (Supplementary Tables 3 and 4; Supplementary Figure 2 and 3). In addition, miR-106a and 130b were strongly upregulated, whereas miR-361, miR-30e* , miR-149, miR-574-3p, and myocyte-enriched miR208a and miR-499 were significantly down-regulated. The down-regulation of miR-208a and miR-499 may reflect their known regulation in cardiac stress ${ }^{7,11}$, or may reflect necrosis present in myocarditis hearts. In support of the latter, other myocyte miRNAs (myomiRs) were either down-regulated (miR-1) or unchanged in hearts of $\mathrm{C} 3 \mathrm{H}$ mice at day 7 , in contrast to $\mathrm{C} 57 \mathrm{BI} / 6$ hearts in which miR-1, miR-133a and miR-133b were up-regulated the same time point (Supplementary Figure 3 and 4).

A number of miRNAs were regulated only in VM-resistant C57BI/6N mice and may be involved in disease susceptibility. Dysregulated miRNAs at day 4 in C57BI/6N included miR-483* miR-411, miR-135*, miR-300 and miR-140. In addition, miR-93* and miR-17* were up-regulated at both day 4 and 7 after infection. Furthermore, miR-361 was consistently found down-regulated in both strains as early as 4 days post-infection and persisting until day 7 (Supplementary Tables 3 and 4).

We selected a set of microRNAs with interesting profiles in either $\mathrm{C} 3 \mathrm{H}$ or $\mathrm{C} 57 \mathrm{BI} / 6 \mathrm{~N}$ VM for qRT-PCR verification of microarray expression profiles. Overall, qRT-PCR measurements confirmed expression patterns observed by microarray analysis, showing significant upregulation of miR-155 (5.9-fold), miR-146b (13.9 fold) and miR-21 (12.8 fold), as well as down-regulation of miR-499 (1.9-fold) (Supplementary Figure 3). 
a human VM versus controls

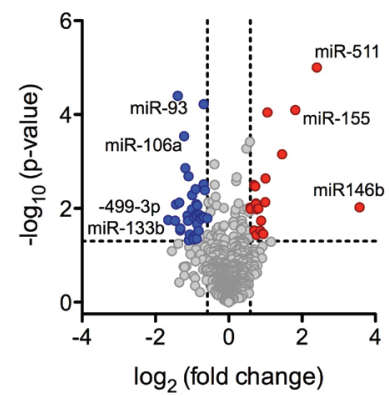

b

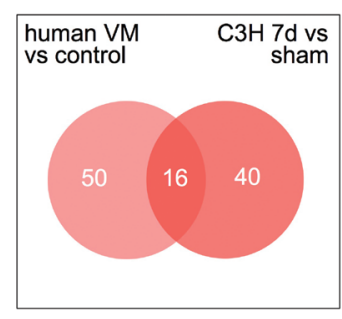

C human versus mouse fold changes

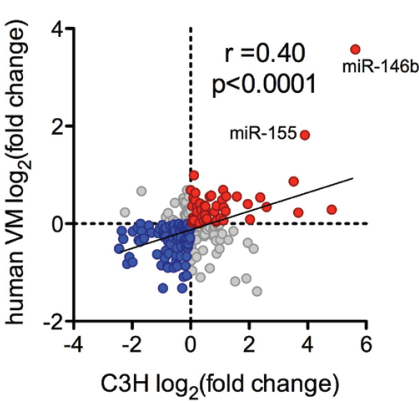

Figure 2. MiRNA expression in human viral myocarditis. (A) Volcano plot of fold changes (log2 values) and $p$ values $\left(\log _{10}\right)$ for individual miRNAs in biopsies of VM patients compared to controls. (B) Venn diagram showing the number and overlap of differentially regulated miRNAs in human versus mouse (C3H 7 days) VM. Fold changes for these miRNAs in both species are plotted in panel (C) correlating significantly $(r=0.40$, $p<0.0001)$.

\section{Cardiac microRNA expression during human viral myocarditis}

In parallel, we analyzed miRNA expression in a set of right ventricular septal biopsies obtained from patients with acute VM and controls. A total of 107 miRNAs were differentially expressed between VM and control hearts of which 21 miRNAs were upand 37 miRNAs were down-regulated by more than 1.5-fold (Figure 2A, Supplementary Figure 5A and Supplementary Table 5). Among the most up-regulated miRNAs were miR-155 (3.5-fold, $p<10^{-4}$ ) and miR-146b (11.9-fold, $p<0.01$ ), besides miR-511 (5-fold), a stimulator of toll-like receptor (TLR) signaling ${ }^{22}$, miR-212 (2.6-fold), involved in cardiomyocyte hypertrophy ${ }^{23}$, and the relatively unknown miR-889 (3.2-fold). Downregulated miRNAs included the inflammatory miR-106a (2.3-fold) and miR-93 (1.6-fold), and miR-361-3p (2.3-fold).

We compared mouse and human miRNA profiles during VM. Out of the 107 miRNAs differentially expressed in human VM, 66 miRNAs could be matched to their mouse homologues, of which 16 were also differentially expressed during mouse VM (Figure 2B-C, Supplementary Figure 5B and Supplementary Table 5). These 16 miRNAs included miR-155, miR-146b, miR-21, and miR-501-3p (consistently up-regulated) and miR-103a, miR-107, miR-99b, miR-149, miR-30a*, and miR-30e* (consistently down-regulated).

Interestingly, the miR-499(-5p) was significantly down-regulated in mouse, but not in human VM, while its opposite strand miR-499-3p was the most down-regulated miRNA in humans (3.1-fold, $\mathrm{p}<0.05$ ) (Supplementary Figure $3 \mathrm{C}$ and $5 \mathrm{C}$ ). MyomiRs that were regulated in humans included miR-133a and miR-133b, which were both significantly down-regulated (Supplementary Table 5). In general, miRNAs with a role in inflammation were dysregulated most robustly while myomiRs were predominantly down-regulated during VM. 
MiR-155 is expressed by infiltrating inflammatory cells in both mouse and human VM

Since miR-155 was strongly and significantly up-regulated in hearts of both VM-susceptible mice and VM patients, we focused on its expression and role in the pathogenesis of VM. To address which cells produce miR-155 during VM, in situ hybridization (ISH) was performed on sections of mouse hearts and human cardiac biopsies. Perinuclear miR-155 staining was observed specifically in inflammatory patches, while no in situ signal was detected in adjacent myocardium. When hybridization was performed with a scrambled control probe no signal was detected, indicating that miR-155 detection was sequencespecific (Figure 3A and Supplementary Figure 6). To learn whether miR-155 preferentially localized in macrophages or T lymphocytes we stained sequential sections for miR-155, CD45, Mac3 and CD3 (Figure 3B). Staining for miR-155 co-localized with CD45-positive leukocytes, including many Mac3-positive macrophages and CD3-positive T lymphocytes. Double staining for miR-155 and CD3 showed that miR-155 signal in infiltrating T lymphocytes was of comparable intensity as in surrounding macrophages (Supplementary Figure $3 \mathrm{C}$ ). These findings are consistent with previous studies showing specificity of miR-155 for hematopoietic cells and up-regulation during activation of virtually all leukocyte subtypes, including macrophages and T cells $s^{13,15,24,25}$.

a

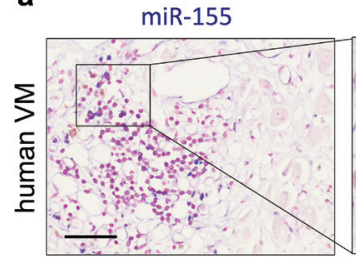

b

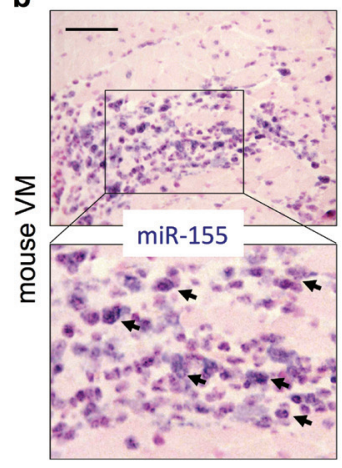

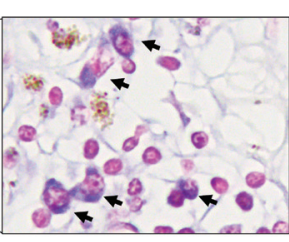
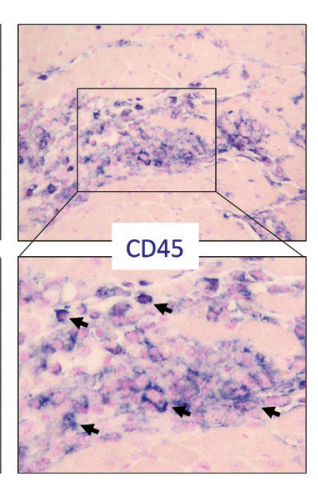

C miR-155/CD3
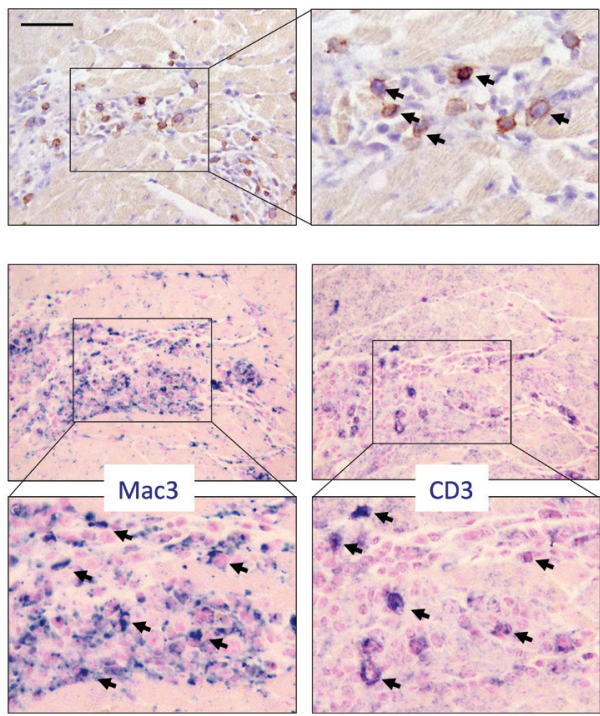



Figure 3. MiR-155 is expressed by infiltrating immune cells during VM. (A) In situ hybridization of miR155 (blue) on a left ventricular biopsy of human VM, showing blue cellular staining in inflammatory patches but not in adjacent myocardium. Panel (B) shows consecutive mouse cardiac ventricular sections (C3H day 7) stained in blue for miR-155, or for markers of leukocytes (CD45), macrophages (Mac3), or T lymphocytes (CD3). The pattern of miR-155 positivity closely resembles that of CD45-positive leukocytes, including many macrophages and also T cells. (C) Co-staining for miR-155 (blue) and CD3 (brown) reveals comparable miR155 staining intensity in T lymphocytes versus other immune cells. Scale bars: $100 \mu \mathrm{m}$. 
To compare miR-155 expression in inflammatory cells to that of cardiac resident cells, we measured miR-155 in a cell panel including neonatal (rat) cardiomyocytes and fibroblasts, mouse endothelial cells (SVEC), mouse bone-marrow derived macrophages (BMM) and splenic T lymphocytes. Cells were incubated for 24 hours with pro-inflammatory stimuli to recapitulate the cardiac inflammatory environment: TNF- $\alpha$ for myocytes, fibroblasts and endothelial cells, LPS for BMM and concanavalin A for T cells. Expression of miR-155 was most pronounced in activated macrophages and T lymphocytes (over 100-fold higher compared to cardiomyocytes; Supplementary Figure 6C). Expression of miR-155 was low in cardiomyocytes, endothelial cells and fibroblasts at baseline, and only induced in fibroblasts by TNF- $\alpha$ stimulation. In conclusion, miR-155 is mainly localized in inflammatory cells during $\mathrm{VM}$ in both mice and humans.

\section{LNA-antimiR inhibits miR-155 function in vivo}

We next asked whether the known inflammatory effects of miR-155 contribute to a beneficial immune response or to adverse inflammation and cardiac injury during VM. Therefore, we inhibited miR-155 in vivo in inflammation-susceptible $\mathrm{C} 3 \mathrm{H}$ mice during the first 7 days of VM. Three individual doses of LNA-antimiR targeting mouse miR-155 or LNA control were administered on days 1, 4 and 6 after CVB3 inoculation to continuously inhibit miR-155 throughout the hematopoietic proliferative response (Figure 4A). Northern blotting showed marked induction of miR-155 during VM and revealed that mature miR-155 was sequestered in a heteroduplex by the LNA-antimiR-155, as evidenced by a band shift of the mature miR-155 (Figure 4B). qRT-PCR for miR-155 confirmed significant knockdown in the inflamed heart $(72 \%, \mathrm{p}<0.001)$. Knockdown was significant but modest in sham hearts $(30 \%, p<0.01)$ and more pronounced in sham spleens that have a high miR-155 expression (Supplementary Figure 7A and B). We verified functional cardiac miR-155 inhibition in an unbiased way using Affymetrix microarrays which showed that mRNAs with canonical 3' UTR seed-match sites for miR-155 were de-repressed in VM animals treated with LNA-anti-155 compared to those from LNA control treated hearts (Figure 4D and Supplementary Figure 7C). Significantly up-regulated genes included validated miR-155 targets such as Activation Induced Cytidine Deaminase (AID), and the transcription factors $\mathrm{BACH}$ 1, Jumonji AT rich interactive domain 2 (JARID2) and CCAAT/enhancer binding protein beta (C/EBP- $\beta$ ). In agreement, unsupervised clustering could differentiate LNA-anti-155 versus LNA scrambled-treated animals with VM but not sham animals (Figure 4E). To investigate antimiR biodistribution, we injected a FAM-labeled fluorescent LNA-antimiR-155 in a parallel group of animals, and could detect its accumulation in cardiac myocytes and endothelial borders as well as in circulating monocytes, lymphocytes and neutrophils (Figure $4 F$ and G). Together, these data confirm that miR-155 was inhibited by LNA-anti-155 treatment in VM animals.

\section{Inhibition of miR-155 reduces cardiac injury and inflammation during VM}

At day 7 after infection, miR-155 inhibition significantly reduced cardiac inflammation and necrosis (Figure 5A and B). In agreement, miR-155 inhibition significantly attenuated heart weight increase during VM, a marker of cardiac inflammatory edema (Figure $5 \mathrm{C}$ and 

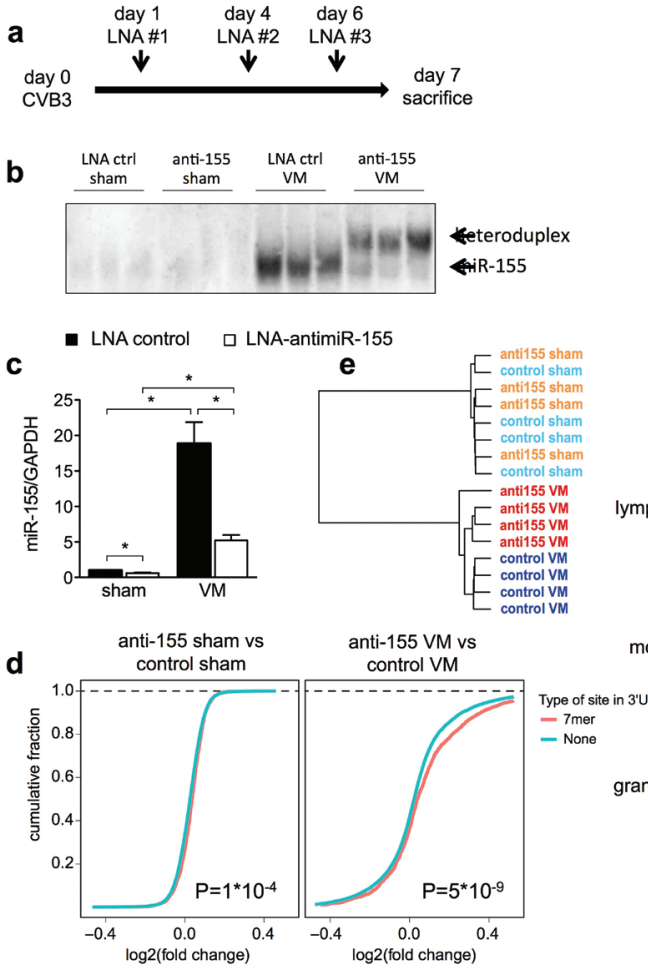

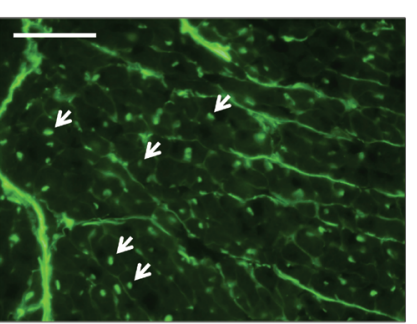

g

lymphocytes

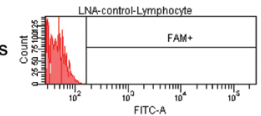

LNA-unlabeled

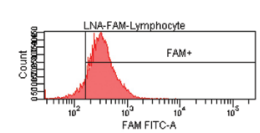

monocytes
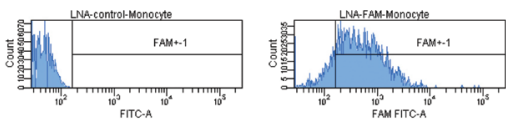

ranulocytes
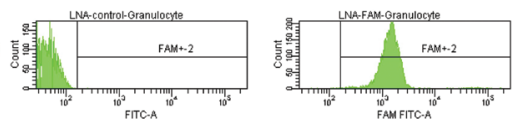

Figure 4. LNA-antimiR effectively inhibits miR-155 during VM. (A) Study design: LNA-antimiR oligonucleotide targeting miR-155 was administered by three intravenous injections throughout the first 7 days of CVB3-induced viral myocarditis. (B) Northern blot analysis shows duplex formation between the LNA-antimiR-155 and mature miR-155, in agreement with decreased miR-155 levels detected by GPCRRT $(C)$ in sham ( $40 \%, p<0.05, n=6$ per group) and VM animals ( $75 \%, p<0.05, n=11$ per group). (D) Derepression of mRNAs with predicted 7-mer miR-155 seed sites in sham and VM animals after miR-155 inhibition with LNA-antimiR-155. Cumulative distributions of mRNA changes between antimiR-155- and LNA control-treated hearts are shown, demonstrating de-repression of miR-155 targets in VM animals but only modestly/not in sham animals after antimiR treatment (one-sided Kolmogorov-Smirnov test; VM: $p=4.7^{*} 10^{-9}$ and sham: $\left.p=1.1^{*} 10^{-4}\right)$. In line, unsupervised hierarchical clustering (E) of experimental groups using microarray expression data could separate VM but not sham groups based on antimiR-treatment, reflecting the more prominent role for miR-155 in the inflamed versus normal hearts. (F) FAM-labeled fluorescent LNA-antimiR accumulates in cardiac nuclei (white arrows) and endothelial linings, as well as in all major hematopoietic lineages present in circulating blood, as shown by flow cytometry. Scale bar: $25 \mu \mathrm{m}$. ${ }^{*} P<0.05$

Supplementary Table 6). Suppression of miR-155 additionally reduced body weight loss associated with systemic illness, as well as the numbers of circulating leukocytes induced by CVB3 infection (Figure 5D and E). We next analyzed the effect of miR-155 inhibition on cardiac recruitment of inflammatory cells using immunohistochemistry. MiR-155 inhibition significantly reduced the number of CD11b-stained monocyte-macrophages and Ly6-stained neutrophils and reduced CD3-stained T cells by trend ( $p=0.09$ ) (Figure 6 A-B and Supplementary Figure $8 \mathrm{~A}$ ). These findings were in keeping with reduced transcripts of CD11b, Ly6G and CD3 (Supplementary Figure 8B), and a more than 50\% reduction of the cytokines interferon 
beta (IFN- $\beta$ ), IFN- $\gamma$, interleukin-6 (IL-6) and tumor necrosis factor alpha (TNF- $\alpha$ ) (Figure 6C and Supplementary Figure $8 \mathrm{C}$ ). The expression of the anti-inflammatory cytokine II-10 as well as the markers for alternatively activated 'anti-inflammatory' macrophages Arginase and YM-1 were markedly reduced by miR-155 inhibition, while II-17 levels were around the detection limit and thus too low for reliable quantification. Finally, cardiac CVB3 genomes were lower in LNA-antimiR-155 treated hearts as compared to controls (Supplementary Figure 8C).

\section{a}
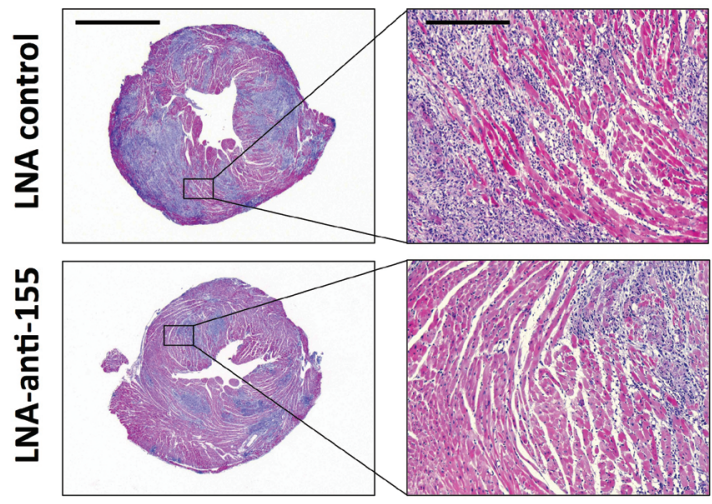

- LNA control $\quad \square$ LNA-antimiR-155

\section{b}

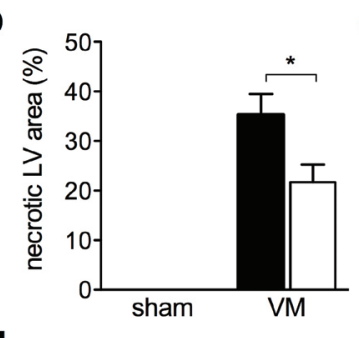

d

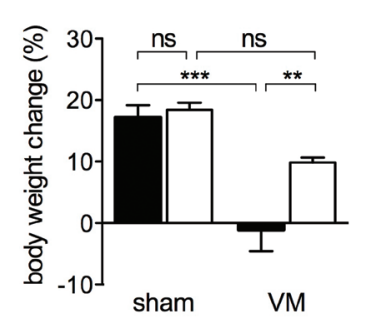

C

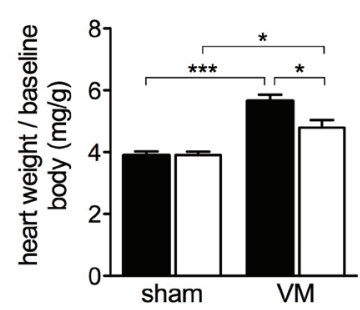

$\mathbf{e}$

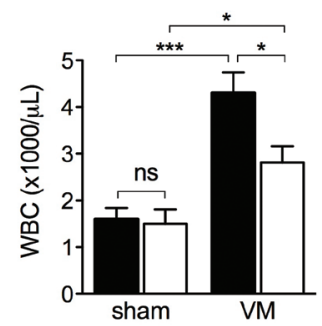

Figure 5. Inhibition of miR-155 reduces myocardial damage in viral myocarditis. $(A, B)$ Left ventricular H\&E stainings at day 7 of CVB3-induced VM reveal reduced cardiomyocytes necrosis after miR-155 inhibition (scale bar: left $2 \mathrm{~mm}$, inset $200 \mu \mathrm{m}$ ) (LV necrotic area quantified in B: LNA-scrambled: 35.3\% versus LNAantimiR-155 19.7\%), in line with a reduced increase in heart weight caused by inflammatory edema (C). Systemically antimiR-155 treatment ameliorated the body weight loss observed in control-treated VM animals (D) and reduced the circulating leukocytosis (LNA-control $4.3 * 10^{9} \mathrm{cells} / \mathrm{L}$ versus LNA-antimiR-155 $2.0^{*} 10^{9}$ cells $/ \mathrm{L}, \mathrm{p}<0.05$ ) (E); WBC: circulating white blood cell count. ( $n=6$ per sham group and $n=11$ per VM group). ${ }^{*} \mathrm{P}<0.05,{ }^{*} \mathrm{P}<0.01$, ${ }^{* *} \mathrm{P}<0.001$. 
To better characterize the effects of miR-155 inhibition on immune cell subtypes, we performed flow cytometry on the cardiac immune cell fraction at day 7 of VM. Flow cytometry revealed that LNA-antimiR-155 treatment markedly reduced the activation of CD4+ and CD8+ T lymphocytes (Figure 7 and Supplementary Table 7). The percentages of naïve (CD62 $\mathrm{L}^{\text {high }} \mathrm{CD} 44^{\text {low) }} \mathrm{T}$ cells in LNA-antimiR-155-treated versus LNA control-treated hearts were $21.7 \%$ versus $4.2 \%$, and $26.6 \%$ versus $10.0 \%$ for $\mathrm{CD}_{4}{ }^{+}$and $\mathrm{CD}^{+} \mathrm{T}$ cells, respectively. Proportions of Foxp ${ }^{+} \mathrm{CD} 25^{+}$regulatory $\mathrm{T}$ cells did not differ between treatment groups. Flow cytometry confirmed that miR-155 inhibition suppressed infiltrating monocytes, without affecting the proportion of pro-inflammatory Ly6 $C^{\text {high }}$ monocytes.

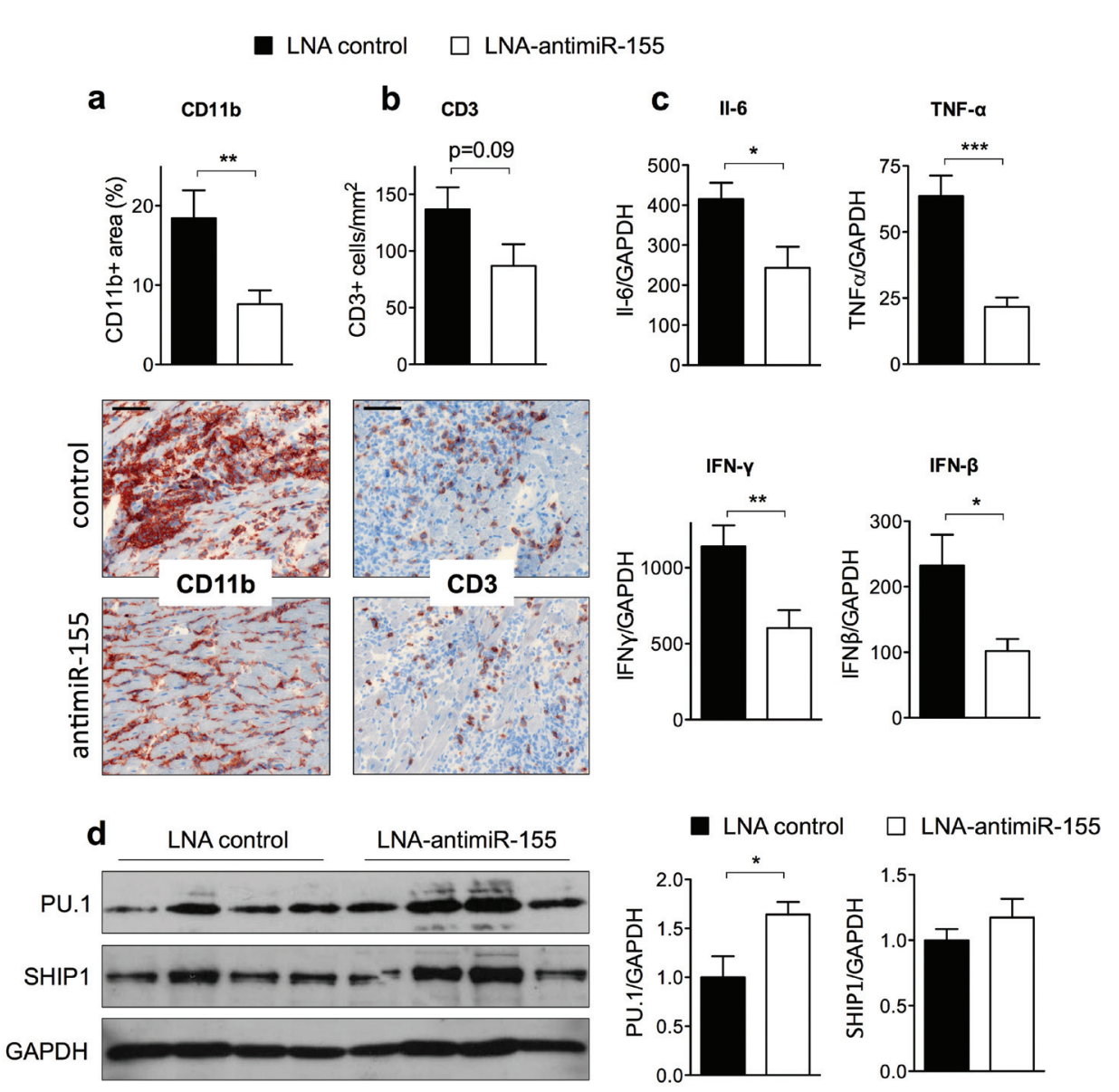

Figure 6. Inhibition of miR-155 reduces cardiac immune cell infiltration and de-represses the direct miR-155 target PU.1 in viral myocarditis. MiR-155 inhibition reduced cardiac infiltration of CD11bimmunoreactive monocyte-macrophages (A) caused a trend towards reduction in CD3+ T cells numbers

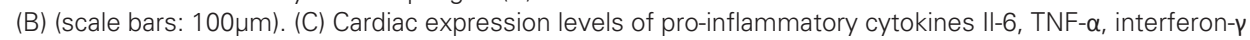
(IFN- $\gamma$ ) and IFN- $\beta$ were suppressed by LNA-antimiR-155. (VM day 7: $n=11$ per group for all analyses). (D) Western blots for the direct miR-155 targets PU.1 and SHIP1 in the cardiac immune fraction during myocarditis, showing that miR-155 inhibition significantly de-repressed PU.1 and increased SHIP1 levels by trend ( $n=4$ per group). ${ }^{*} P<0.05,{ }^{*} P<0.01,{ }^{* * *} P<0.001$. 
Together, these data suggest that the attenuation of the immune response after miR-155 suppression is broad and involves both the innate and adaptive immune system, rather than supporting a preferential activation of anti-inflammatory cells or signaling.

\section{AntimiR-155 treatment de-represses the direct miR-155 target PU.1 in cardiac inflammatory cells}

We next performed western blots on previously confirmed direct miR-155 targets, whose de-repression in leukocytes could explain protection by antimiR treatment. Expression of PU.1 (an inhibitor of dendritic cell antigen presentation to T cells26), and SHIP1 (a regulator of myeloid proliferation ${ }^{27,}{ }^{28}$ ) was hardly detectable in sham hearts but increased with severity of inflammation, in agreement with their expression by inflammatory cells (Supplementary Figure 9). Since the extent of cardiac inflammation might thus confound protein levels in whole-hearts, we focused on protein levels in
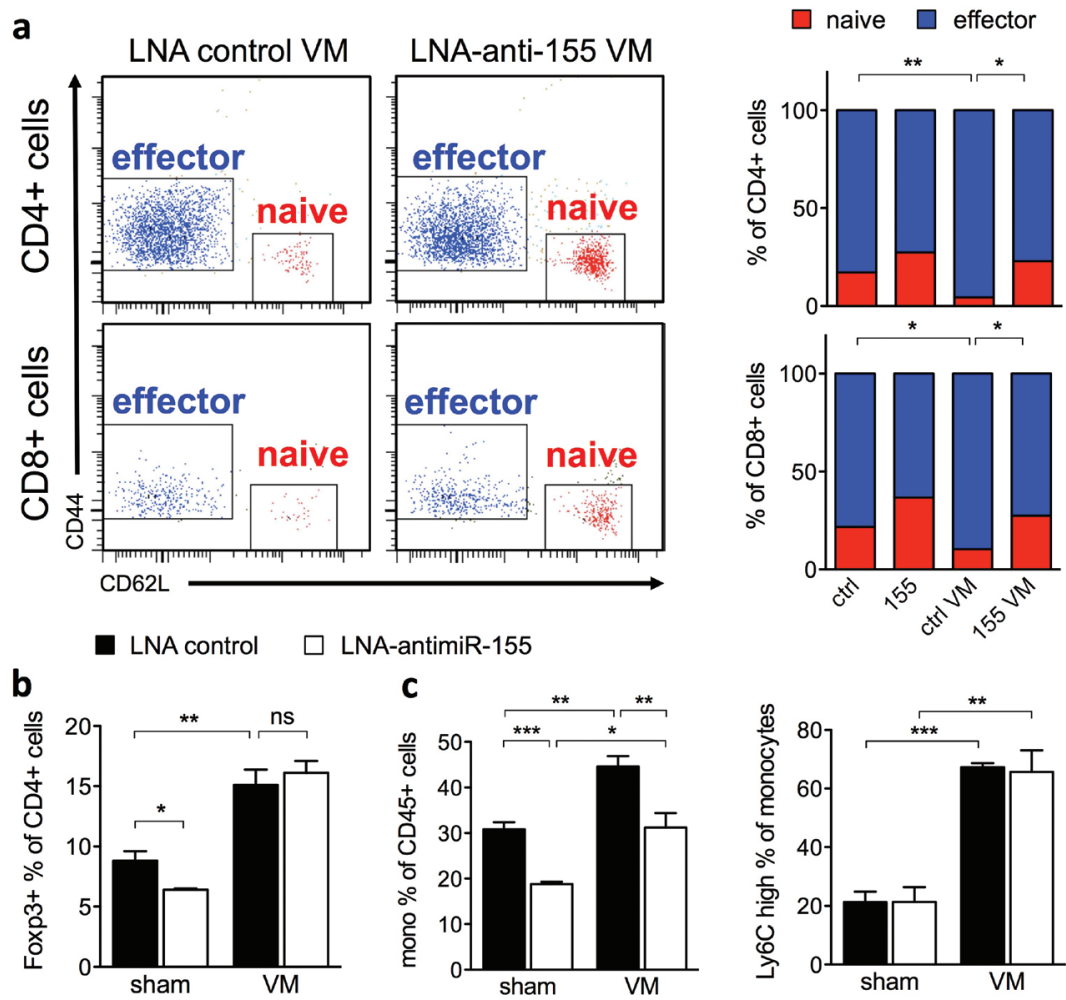

LNA-antimiR-155
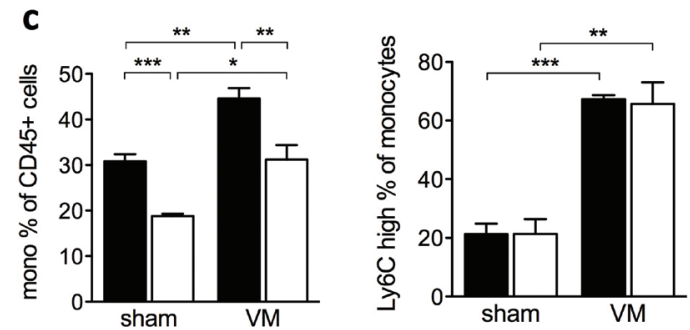

Figure 7. MiR-155 inhibition suppresses T lymphocyte activation in myocarditis hearts. (A) Flow cytometry of the cardiac immune fraction. MiR-155 inhibition significantly increased the percentage of naïve (CD62 $\mathrm{L}^{\text {high }} \mathrm{CD} 44^{\text {low }}$ ) versus effector/effector-memory (CD62 $\mathrm{L}^{\text {low }} \mathrm{CD} 44^{\text {high) }} \mathrm{CD} 4^{+}$and $\mathrm{CD} 8^{+} \mathrm{T}$ lymphocytes in myocarditis hearts (LNA-antimiR-155 vs LNA control: $21.7 \%$ vs $4.2 \%$ and $26.6 \%$ vs $10.0 \%$ for CD4+ and $\mathrm{CD}^{+}$, respectively). (B) The amount of Foxp $3^{+} \mathrm{CD} 25^{+}$regulatory $\mathrm{T}$ cells was not different between treatment groups. (C) MiR-155 inhibition significantly reduced the percentage monocytes of the cardiac infiltrates, but did not affect the proportion of pro-inflammatory Ly6C high monocytes $(n=4$ per sham group and $n=8$ per VM group). ${ }^{*} P<0.05$, ${ }^{*} P<0.01,{ }^{*}{ }^{*} P<0.001$. 
LNA control $\quad \square$ LNA-antimiR-155

a

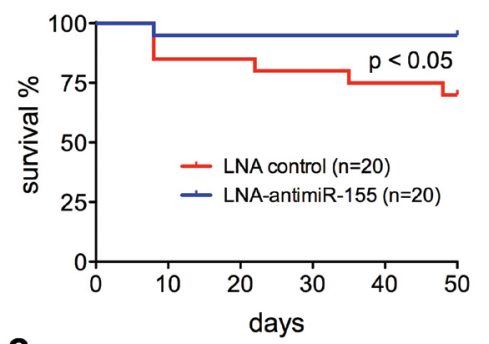

C

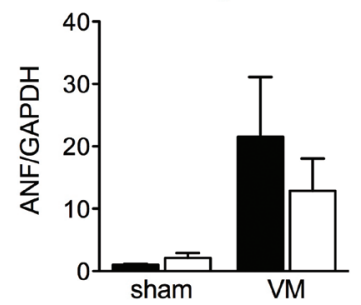

b
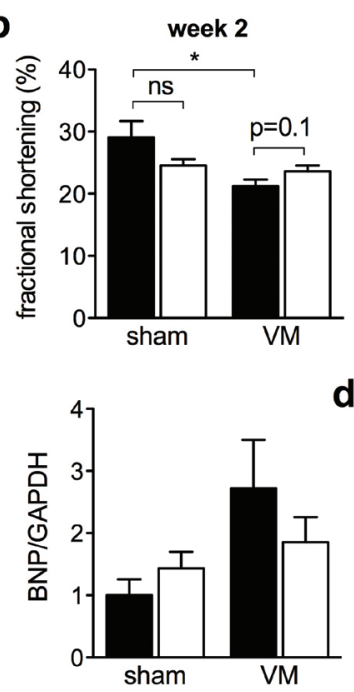

d
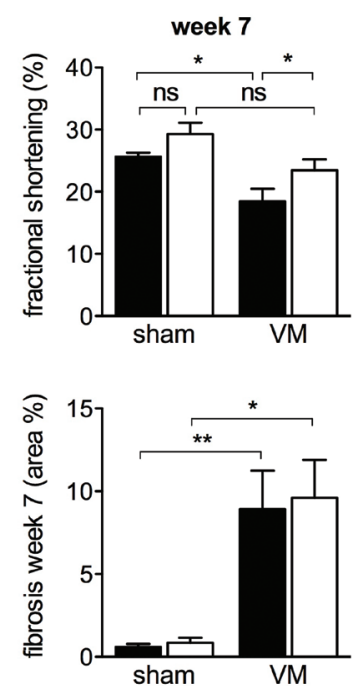

Figure 8. MiR-155 inhibition protects against mortality and systolic dysfunction in viral myocarditis. (A) Kaplan-Meier curve showing reduced mortality after miR-155 inhibition compared to controls $(1 / 20$ versus $6 / 20$, respectively; $p<0.05$ ). (B) Fractional shortening was decreased in $V M$ control hearts at weeks 2 and 7 compared to shams, but not in miR-155 inhibited VM hearts. After 7 weeks, fractional shortening was significantly higher in LNA-antimiR-155 versus LNA control animals $(23.4 \%$ versus $18.4 \%$, respectively; $p<0.05$ ). (C) The cardiac increase in fetal gene markers in surviving animals was attenuated by miR-155 inhibition, although this difference was not statistically significant. We did not observe differences in cardiac collagen deposition between antimiR treatment groups (D). ( $n=6$ per sham group and $n=20$ per VM group). ${ }^{*} P<0.05,{ }^{*} P<0.01$.

isolated cardiac immune fractions. Protein expression of PU.1 was significantly increased in cardiac leukocytes after inhibition of miR-155, while SHIP1 de-repression was not statistically significant (Figure 6D).

\section{Inhibition of miR-155 inhibition does not directly affect viral replication or myocyte viability}

To investigate whether miR-155 inhibition directly impacts CVB3 load or early cytokine signaling by cardiac resident cells, we sacrificed animals at day 4 after infection, before onset of inflammation. We found no significant differences in viral load between treatments in the heart, spleen or liver (Supplementary Figure 10A). Also, miR-155 inhibition did not affect cardiac expression of cytokines including TNF- $\alpha$, II-6, II-12a, II-10, IFN- $\beta$, IFN- - , and the chemokine macrophage chemo-attractant protein-1 (MCP-1) at this time point (Supplementary Figure 10B).

In addition, in vitro knockdown of miR-155 prior to CVB3 infection did not affect cell death, caspase 3 cleavage, or viral replication in neonatal rat cardiomyocytes (Supplementary Figure 11). These data support that the adverse effects of miR-155 during VM are leukocyteautonomous and independent from viral load in cardiomyocytes, myocyte viability, or cardiac cytokine signaling prior to infiltration by inflammatory cells. 


\section{Prolonged inhibition of miR-155 during myocarditis improves survival and cardiac function}

To assess the long-term effects of miR-155 inhibition during VM, mice were followed for 7 weeks after CVB3 infection. Treatment with miR-155 inhibitors significantly reduced mortality (1/20 versus 6/20) (Figure 8 and Supplementary Table 6). In addition to decreasing mortality, miR-155 inhibition improved cardiac function in animals surviving after 7 weeks (fractional shortening 23.4\% versus 18.4\%) (Figure 8 and Supplementary Table 8). RNA analysis revealed complete viral elimination in both groups and a trend to reduced induction of cardiac fetal gene markers (ANF, BNP), although this was not statistically significant. Cardiac fibrosis was similar in both groups after 7 weeks though early fibrosis at 1 week was reduced by miR-155 inhibition (Figure 8 and Supplementary Figure 12). In conclusion, miR-155 inhibition improved mortality and cardiac function after long-term treatment.

\section{Discussion}

This study is the first to investigate microRNA (miRNA) expression profiles related to cardiac virus infection and resulting inflammation in both mice and humans. We identify microRNA-155 as a key contributor to exaggerated antiviral surveillance.

Two of the most important questions in myocarditis research are (1) why infection by cardiotropic viruses triggers myocarditis in some patients while remaining asymptomatic in others, and (2) what signaling pathways contribute to cardiac injury during the inflammatory response, since distinct components of the inflammatory response can contribute to either efficient viral elimination and cardiac protection, or to irreversible cardiac damage and functional deterioration in $\mathrm{VM}^{2}, 29,30$. The current study addresses these questions by establishing a set of susceptibility- and inflammation-related miRNAs in mice, and identifying miR-155 as a contributor to adverse inflammation during VM both in mice and human VM. Moreover, our results suggest therapeutic opportunities by targeting miR-155 in mice to limit cardiac injury and mortality in VM.

The pro-inflammatory effects of miR-155 in B and T lymphocytes as well as macrophages and dendritic cells have been well established. MiR-155 expression is induced during activation of both macrophages and lymphocytes ${ }^{14,15}$. In lymphocytes, miR-155 promotes Th1 responses as well as germinal center formation ${ }^{13,14}$, while favoring proliferation and pro-inflammatory cytokine secretion in myeloid cells ${ }^{27,31}$. MiR-155 also mediated crosstalk between innate and adaptive immune cells, as miR-155 deficient dendritic cells are less capable of activating $T$ cells ${ }^{14,26}$. This multitude of pro-inflammatory effects of miR-155 on both the innate and adaptive immune system is associated with a variety of anti-inflammatory protein targets, including proteins Suppressor of Cytokine Secretion 1 (SOCS1) ${ }^{32}, 33$, SH2containing Inositol-5-phosphatase (SHIP1) ${ }^{27}$, AID ${ }^{34}$, and the transcription factors PU. $1^{35}$ and $\mathrm{C}-\mathrm{MAF}^{14}$. In line with these effects, miR-155 was shown to be protective during experimental autoimmune encephalitis $(E A E)^{36} 37$ and rheumatoid arthritis ${ }^{28}$.

Most of the immune mechanisms controlled by miR-155 are important in the pathogenesis of VM. However, because VM pathogenesis involves viral pathogens, protective effects 
of anti-inflammatory manipulations are not trivial. In the current study, pharmacological inhibition of miR-155 reduced both cardiac damage and mortality during VM, indicating that miR-155 signaling contributes to an adverse rather than beneficial immune activation in the context of VM. AntimiR treatment reduced cardiac numbers of monocyte-macrophages and inhibited $\mathrm{T}$ lymphocyte activation, in parallel with down-regulation of both pro- and anti-inflammatory cytokines including TNF- $\alpha, I-6$, IL-10 and IFN- $-\gamma$ during the inflammatory phase. The increased percentage of naïve T cells in LNA-antimiR-155-treated animals was in line with the de-repression of PU.1, a direct miR-155 target that inhibits antigen presentation to lymphocytes in dendritic cells ${ }^{26}$. Cardiac protection by miR-155 inhibition was likely immune-cell autonomous, since miR-155 inhibition did not affect viral load, myocyte viability, or cytokine signaling by cardiac resident cells prior to infiltration by immune cells. Our results therefore suggest that therapeutic targeting of miR-155 may benefit myocarditis patients by dampening inflammation. Of note, miR-155 has been described to target the angiotensin II type 1 receptor (AT1R) ${ }^{38}$. Although we did not observe increased fibrosis after miR-155 inhibition, clinical studies are required to exclude shortterm side effects of antimiR-155 treatment.

Beside miRNA-155, we identified a set of miRNAs that is consistently up-regulated during acute inflammation of both human and mouse VM, including miR-155, miR-146b and miR21 , which all have central functions in immune activation and inflammation ${ }^{21,39}$ and the less well-known miR-130b, a virus-induced inhibitor of target cell apoptosis in humans ${ }^{40}$. MiR106a, which was up-regulated in both $\mathrm{C} 3 \mathrm{H}$ and $\mathrm{C} 57 \mathrm{BI} / 6 \mathrm{~N}$ at day 7 post-infection. MiR-106a has been shown to target interleukin-10, a central regulator of the immune system ${ }^{41}$, while the expression of miR-106a itself is regulated by the transcription factors Egr-1 and Sp-1, both key in cardiac processes ${ }^{41}$. Immune functions have also been described for consistently down-regulated candidates: miR-107 is down-regulated in multiple cell types in response to TLR-4 signaling and is required to facilitate macrophage adhesion42 whereas inhibition of miR-99b reduces dendritic cell differentiation ${ }^{22}$. In addition, we identified a set of miRNAs exclusively associated with viral infection in resistant C57BI6/N mice, including miR-483*, miR-411, miR-135*, miR-300 and miR-140, miR-93* and miR-17*. While these miRNAs are relatively unknown, their likely involvement in the primary response to viral infection and disease susceptibility invites future studies.

In conclusion, VM induces marked miRNA expression changes in the mouse and human heart. MiRNA-155 is up-regulated in myeloid and lymphoid inflammatory cells during acute $\mathrm{VM}$ and is a mediator of adverse cardiac immune activation. In vivo inhibition of microRNA-155 after CVB3 infection attenuates myocardial inflammation and necrosis, reduces mortality and improves cardiac function. As such, this study identifies miRNA-155 as a novel therapeutic target for treatment of viral myocarditis.

\section{Acknowledgements}

We are grateful to Kevin Custers, Laura de Rijck, and Casper Eurlings for excellent support. 


\section{References}

1. Cooper LT, Jr. Myocarditis. N Engl J Med. 2009;360:1526-1538

2. Esfandiarei M, McManus BM. Molecular biology and pathogenesis of viral myocarditis. Annual review of pathology. 2008;3:127-155

3. Bartel DP. Micrornas: Target recognition and regulatory functions. Cell. 2009;136:215-233

4. Chen JF, Murchison EP, Tang R, Callis TE, Tatsuguchi M, Deng Z, Rojas M, Hammond SM, Schneider MD, Selzman CH, Meissner G, Patterson C, Hannon GJ, Wang DZ. Targeted deletion of dicer in the heart leads to dilated cardiomyopathy and heart failure. Proceedings of the National Academy of Sciences of the United States of America. 2008;105:2111-2116

5. Small EM, Olson EN. Pervasive roles of micrornas in cardiovascular biology. Nature. 2011;469:336-342

6. da Costa Martins PA, Salic K, Gladka MM, Armand AS, Leptidis S, el Azzouzi H, Hansen A, Coenen-de Roo CJ, Bierhuizen MF, van der Nagel R, van Kuik J, de Weger R, de Bruin A, Condorelli G, Arbones ML, Eschenhagen T, De Windt LJ. Microrna-199b targets the nuclear kinase dyrk1a in an auto-amplification loop promoting calcineurin/nfat signalling. Nature cell biology. 2010;12:1220-1227

7. van Rooij E, Sutherland LB, Qi X, Richardson JA, Hill J, Olson EN. Control of stress-dependent cardiac growth and gene expression by a microrna. Science. 2007;316:575-579

8. Thum T, Gross C, Fiedler J, Fischer T, Kissler S, Bussen M, Galuppo P, Just S, Rottbauer W, Frantz S, Castoldi M, Soutschek J, Koteliansky V, Rosenwald A, Basson MA, Licht JD, Pena JT, Rouhanifard SH, Muckenthaler MU, Tuschl T, Martin GR, Bauersachs J, Engelhardt S. Microrna-21 contributes to myocardial disease by stimulating map kinase signalling in fibroblasts. Nature. 2008;456:980-984

9. van Rooij E, Sutherland LB, Thatcher JE, DiMaio JM, Naseem RH, Marshall WS, Hill JA, Olson EN. Dysregulation of micrornas after myocardial infarction reveals a role of mir-29 in cardiac fibrosis. Proc Natl Acad Sci U S A. 2008;105:13027-13032

10. Fiedler J, Jazbutyte V, Kirchmaier BC, Gupta SK, Lorenzen J, Hartmann D, Galuppo P, Kneitz S, Pena JT, SohnLee C, Loyer X, Soutschek J, Brand T, Tuschl T, Heineke J, Martin U, Schulte-Merker S, Ertl G, Engelhardt S, Bauersachs J, Thum T. Microrna-24 regulates vascularity after myocardial infarction. Circulation. 2011;124:720730

11. Wang JX, Jiao JQ, Li Q, Long B, Wang K, Liu JP, Li YR, Li PF. Mir-499 regulates mitochondrial dynamics by targeting calcineurin and dynamin-related protein-1. Nature medicine. 2011;17:71-78

12. Bonauer A, Carmona G, Iwasaki M, Mione M, Koyanagi M, Fischer A, Burchfield J, Fox H, Doebele C, Ohtani K, Chavakis E, Potente M, Tjwa M, Urbich C, Zeiher AM, Dimmeler S. Microrna-92a controls angiogenesis and functional recovery of ischemic tissues in mice. Science. 2009;324:1710-1713

13. Thai TH, Calado DP, Casola S, Ansel KM, Xiao C, Xue Y, Murphy A, Frendewey D, Valenzuela D, Kutok JL, Schmidt-Supprian M, Rajewsky N, Yancopoulos G, Rao A, Rajewsky K. Regulation of the germinal center response by microrna-155. Science. 2007;316:604-608

14. Rodriguez A, Vigorito E, Clare S, Warren MV, Couttet P, Soond DR, van Dongen S, Grocock RJ, Das PP, Miska EA, Vetrie D, Okkenhaug K, Enright AJ, Dougan G, Turner M, Bradley A. Requirement of bic/microrna-155 for normal immune function. Science. 2007;316:608-611

15. O'Connell RM, Taganov KD, Boldin MP, Cheng G, Baltimore D. Microrna-155 is induced during the macrophage inflammatory response. Proceedings of the National Academy of Sciences of the United States of America. 2007;104:1604-1609

16. Lanford RE, Hildebrandt-Eriksen ES, Petri A, Persson R, Lindow M, Munk ME, Kauppinen S, Orum H. Therapeutic silencing of microrna-122 in primates with chronic hepatitis c virus infection. Science. 2010;327:198-201

17. Elmen J, Lindow M, Schutz S, Lawrence M, Petri A, Obad S, Lindholm M, Hedtjarn M, Hansen HF, Berger U, Gullans S, Kearney P, Sarnow P, Straarup EM, Kauppinen S. Lna-mediated microrna silencing in non-human primates. Nature. 2008;452:896-899 
18. Chow LH, Gauntt CJ, McManus BM. Differential effects of myocarditic variants of coxsackievirus b3 in inbred mice. A pathologic characterization of heart tissue damage. Laboratory investigation; a journal of technical methods and pathology. 1991;64:55-64

19. Jorgensen S, Baker A, Moller S, Nielsen BS. Robust one-day in situ hybridization protocol for detection of micrornas in paraffin samples using Ina probes. Methods. 2010;52:375-381

20. Nuovo GJ. In situ detection of micrornas in paraffin embedded, formalin fixed tissues and the co-localization of their putative targets. Methods. 2010;52:307-315

21. van de Vrie M, Heymans S, Schroen B. Microrna involvement in immune activation during heart failure. Cardiovascular drugs and therapy / sponsored by the International Society of Cardiovascular Pharmacotherapy. 2011;25:161-170

22. Tserel L, Runnel T, Kisand K, Pihlap M, Bakhoff L, Kolde R, Peterson H, Vilo J, Peterson P, Rebane A. Microrna expression profiles of human blood monocyte-derived dendritic cells and macrophages reveal mir-511 as putative positive regulator of toll-like receptor 4. The Journal of biological chemistry. 2011;286:26487-26495

23. Jentzsch C, Leierseder S, Loyer X, Flohrschutz I, Sassi Y, Hartmann D, Thum T, Laggerbauer B, Engelhardt S. A phenotypic screen to identify hypertrophy-modulating micrornas in primary cardiomyocytes. Journal of molecular and cellular cardiology. 2012;52:13-20

24. Landgraf P, Rusu M, Sheridan R, Sewer A, lovino N, Aravin A, Pfeffer S, Rice A, Kamphorst AO, Landthaler M, Lin C, Socci ND, Hermida L, Fulci V, Chiaretti S, Foa R, Schliwka J, Fuchs U, Novosel A, Muller RU, Schermer B, Bissels U, Inman J, Phan Q, Chien M, Weir DB, Choksi R, De Vita G, Frezzetti D, Trompeter HI, Hornung V, Teng G, Hartmann G, Palkovits M, Di Lauro R, Wernet P, Macino G, Rogler CE, Nagle JW, Ju J, Papavasiliou FN, Benzing T, Lichter P, Tam W, Brownstein MJ, Bosio A, Borkhardt A, Russo JJ, Sander C, Zavolan M, Tuschl T. A mammalian microrna expression atlas based on small rna library sequencing. Cell. 2007;129:1401-1414

25. Haasch D, Chen YW, Reilly RM, Chiou XG, Koterski S, Smith ML, Kroeger P, McWeeny K, Halbert DN, Mollison KW, Djuric SW, Trevillyan JM. T cell activation induces a noncoding rna transcript sensitive to inhibition by immunosuppressant drugs and encoded by the proto-oncogene, bic. Cellular immunology. 2002;217:78-86

26. Martinez-Nunez RT, Louafi F, Friedmann PS, Sanchez-Elsner T. Microrna-155 modulates the pathogen binding ability of dendritic cells (dcs) by down-regulation of dc-specific intercellular adhesion molecule-3 grabbing nonintegrin (dc-sign). The Journal of biological chemistry. 2009;284:16334-16342

27. O'Connell RM, Chaudhuri AA, Rao DS, Baltimore D. Inositol phosphatase ship1 is a primary target of mir-155. Proceedings of the National Academy of Sciences of the United States of America. 2009;106:7113-7118

28. Kurowska-Stolarska M, Alivernini S, Ballantine LE, Asquith DL, Millar NL, Gilchrist DS, Reilly J, lerna M, Fraser AR, Stolarski B, McSharry C, Hueber AJ, Baxter D, Hunter J, Gay S, Liew FY, Mclnnes IB. Microrna-155 as a proinflammatory regulator in clinical and experimental arthritis. Proceedings of the National Academy of Sciences of the United States of America. 2011;108:11193-11198

29. Woodruff JF, Woodruff JJ. Involvement of t lymphocytes in the pathogenesis of coxsackie virus b3 heart disease. Journal of immunology. 1974;113:1726-1734

30. Hashimoto I, Komatsu T. Myocardial changes after infection with coxsackie virus b3 in nude mice. British journal of experimental pathology. 1978;59:13-20

31. O'Connell RM, Zhao JL, Rao DS. Microrna function in myeloid biology. Blood. 2011;118:2960-2969

32. Androulidaki A, lliopoulos D, Arranz A, Doxaki C, Schworer S, Zacharioudaki V, Margioris AN, Tsichlis PN, Tsatsanis C. The kinase akt1 controls macrophage response to lipopolysaccharide by regulating micrornas. Immunity. 2009;31:220-231

33. Lu LF, Boldin MP, Chaudhry A, Lin LL, Taganov KD, Hanada T, Yoshimura A, Baltimore D, Rudensky AY. Function of mir-146a in controlling treg cell-mediated regulation of th1 responses. Cell. 2010;142:914-929

34. Teng G, Hakimpour P, Landgraf P, Rice A, Tuschl T, Casellas R, Papavasiliou FN. Microrna-155 is a negative regulator of activation-induced cytidine deaminase. Immunity. 2008;28:621-629

35. Vigorito E, Perks KL, Abreu-Goodger C, Bunting S, Xiang Z, Kohlhaas S, Das PP, Miska EA, Rodriguez A, Bradley A, Smith KG, Rada C, Enright AJ, Toellner KM, Maclennan IC, Turner M. Microrna-155 regulates the generation of immunoglobulin class-switched plasma cells. Immunity. 2007;27:847-859 
36. O'Connell RM, Kahn D, Gibson WS, Round JL, Scholz RL, Chaudhuri AA, Kahn ME, Rao DS, Baltimore D. Microrna-155 promotes autoimmune inflammation by enhancing inflammatory t cell development. Immunity. 2010;33:607-619

37. Murugaiyan G, Beynon V, Mittal A, Joller N, Weiner HL. Silencing microrna-155 ameliorates experimental autoimmune encephalomyelitis. Journal of immunology. 2011;187:2213-2221

38. Martin MM, Lee EJ, Buckenberger JA, Schmittgen TD, Elton TS. Microrna-155 regulates human angiotensin ii type 1 receptor expression in fibroblasts. The Journal of biological chemistry. 2006;281:18277-18284

39. Kumarswamy R, Volkmann I, Thum T. Regulation and function of mirna-21 in health and disease. RNA biology. $2011 ; 8$

40. Yeung ML, Yasunaga J, Bennasser Y, Dusetti N, Harris D, Ahmad N, Matsuoka M, Jeang KT. Roles for micrornas, mir-93 and mir-130b, and tumor protein 53-induced nuclear protein 1 tumor suppressor in cell growth dysregulation by human t-cell lymphotrophic virus 1. Cancer research. 2008;68:8976-8985

41. Sharma A, Kumar M, Aich J, Hariharan M, Brahmachari SK, Agrawal A, Ghosh B. Posttranscriptional regulation of interleukin-10 expression by hsa-mir-106a. Proceedings of the National Academy of Sciences of the United States of America. 2009;106:5761-5766

42. Hennessy EJ, Sheedy FJ, Santamaria D, Barbacid M, O'Neill LA. Toll-like receptor-4 (tIr4) down-regulates microrna-107, increasing macrophage adhesion via cyclin-dependent kinase 6 . The Journal of biologica chemistry. 2011;286:25531-25539 


\section{Supplementary Figures}

a

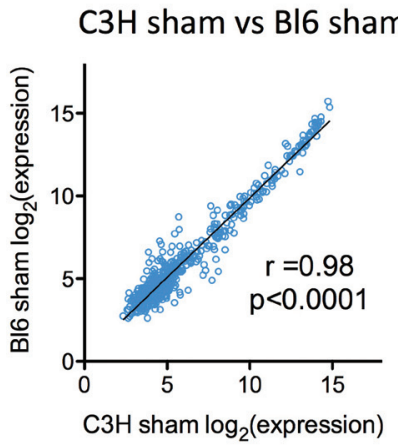

b
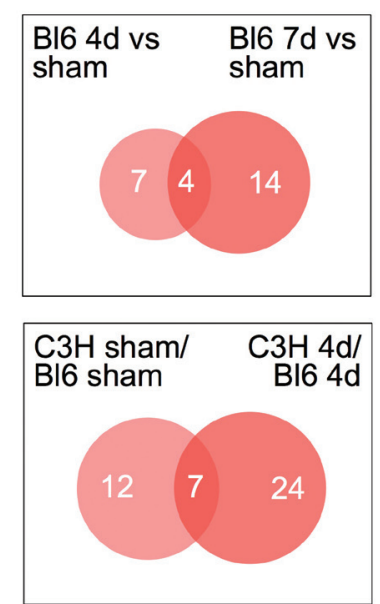

$\mathrm{C} 3 \mathrm{H}$ d4 vs Bl6 d4
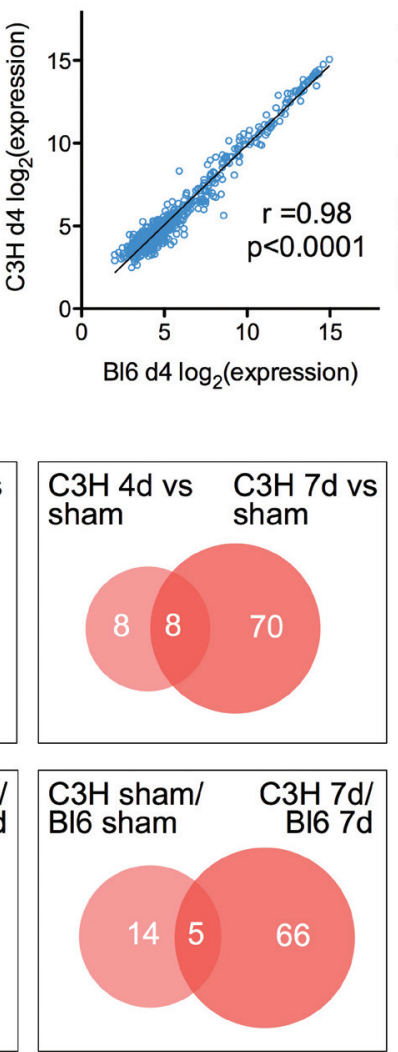

Supplementary Figure 1. MicroRNA (miRNA) expression profiles of viral myocarditis in susceptible (C3H) or non-susceptible (C57BI/6N) mice. (A) Correlation plots of cardiac miRNA expression values in $\mathrm{C} 3 \mathrm{H}$ versus $\mathrm{C} 57 \mathrm{BI} / 6 \mathrm{~N}$ animals in sham animals, and at days 4 and 7 after CVB3 infection. (B) Venn diagrams comparing numbers and overlaps of differentially expressed miRNAs in mice from day 4 to 7 , and differences between mouse strains at days 0,4 , or 7 . 
a

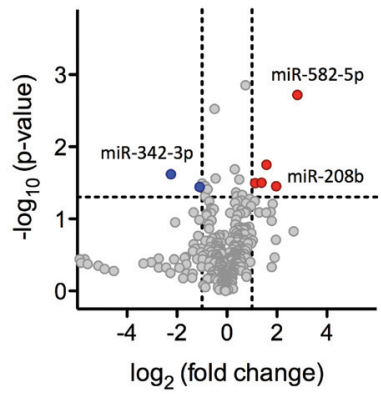

b

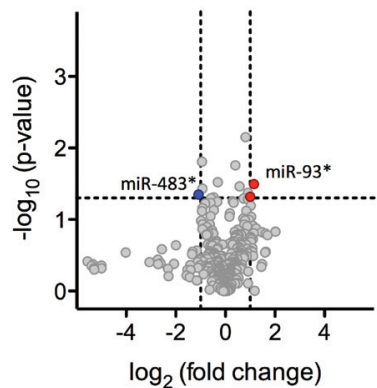

C3H 4d vs Bl6 4d

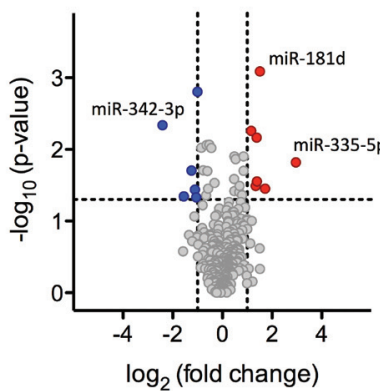

$\mathrm{C} 3 \mathrm{H}: 4 \mathrm{~d}$ vs sham

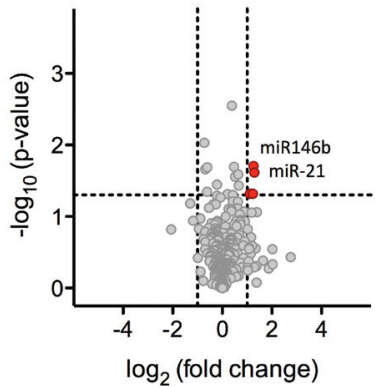

C3H 7d vs Bl6 7d

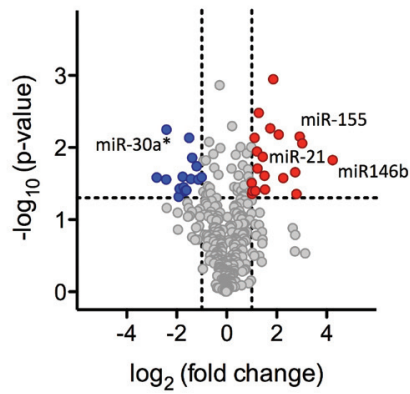

$\log _{2}$ (fold change)

Supplementary Figure 2. Differentially expressed miRNAs in murine viral myocarditis. (A) Volcano plots showing fold changes and p-values of individual miRNAs between C57BI/6N and $\mathrm{C} 3 \mathrm{H}$ mice at time points: sham, day 4 and day 7 after CVB3 infection. (B) Volcano plots of differential miRNA expression between sham and day 4 of either mouse strain. 
a

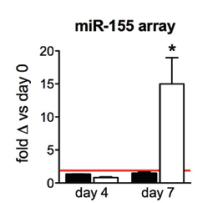

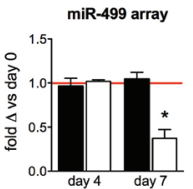
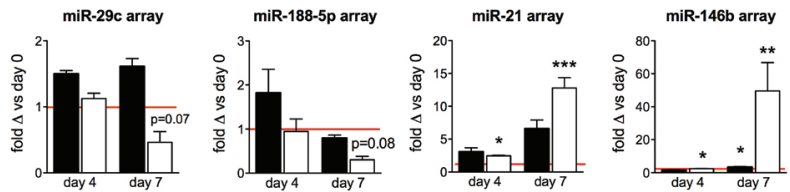

b
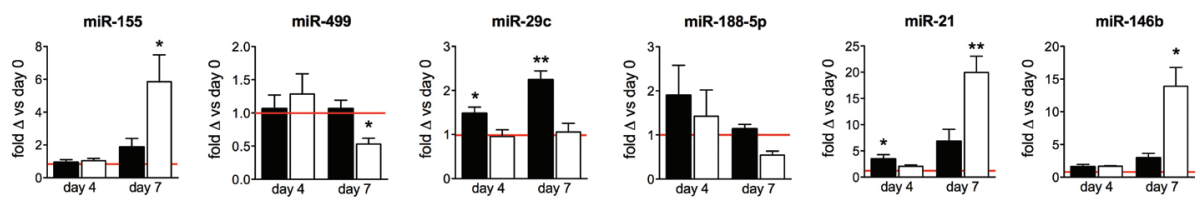

C

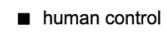

\section{$\square$ human VM}

$\operatorname{miR}-155$

miR-499

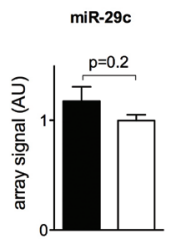

miR-188-5p
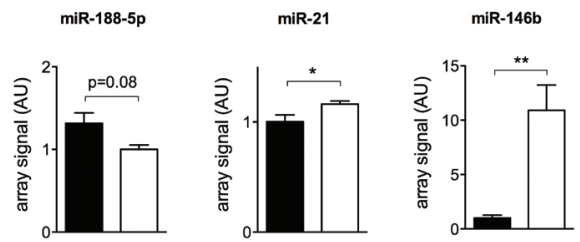

Supplementary Figure 3. Quantitative RT-PCR validation of mouse array results. (A) Array results and (B) qRT-PCR results of selected miRNAs on day 4 or 7 versus sham levels (red line) ( $n=3$ per group), showing reproducibility of the array-identified expression patterns. (C) Human array results for the miRNAs validated in panel $A$ and $B(V M n=4$, controls $n=6)$. ${ }^{*} P<0.05,{ }^{*} P<0.01,{ }^{* *} P<0.001$.

- $\mathrm{C} 57 \mathrm{BI} / 6 \mathrm{~N} \square \mathrm{C} 3 \mathrm{H}$

a
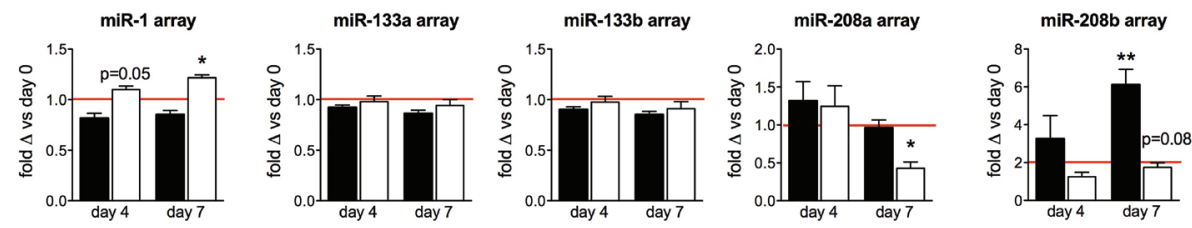

b
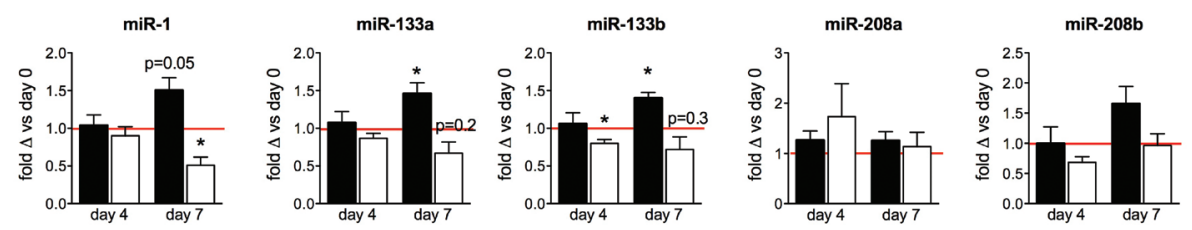

Supplementary Figure 4. Quantitative RT-PCR validation of mouse array results for myomiRs. (A) Array results and (B) qRT-PCR results of selected miRNAs on day 4 or 7 versus sham levels (red line) ( $n=3$ per group). QRT-PCR measurements show that myomiRs are not differentially regulated in $\mathrm{C} 3 \mathrm{H}$ hearts at day 7, with the exception of miR-1, which is significantly down-regulated. In contrast, miR-1, miR-133a and miR-133b are up-regulated in C57BI/6 hearts at day $7 .{ }^{*} \mathrm{P}<0.05,{ }^{*} \mathrm{P}<0.01$. 
a

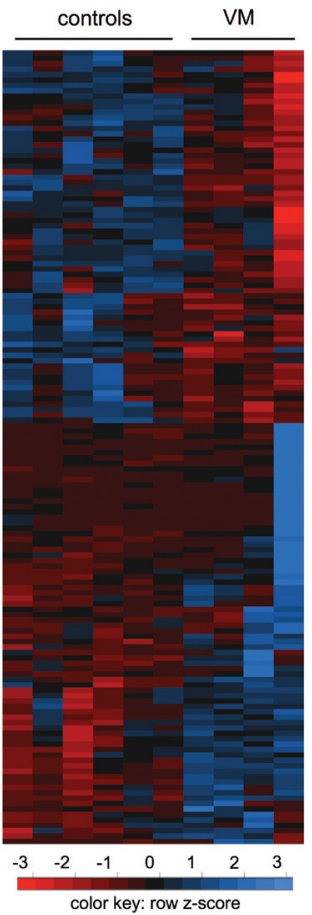

b

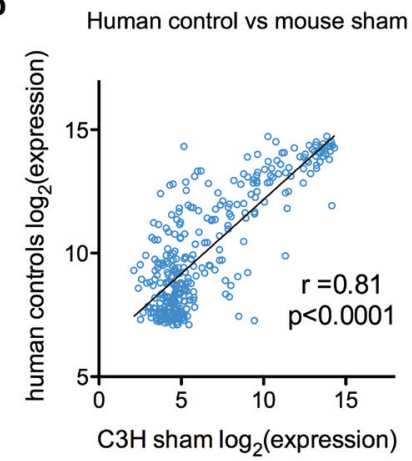

C

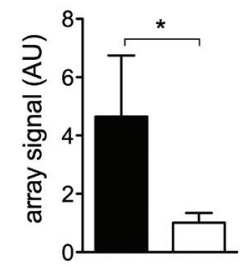

Supplementary Figure 5. MiRNA-expression profiles of human viral myocarditis. (A) Heat map of differentially expressed microRNAs $(p<0.05)$ between left ventricular biopsies of viral myocarditis patients compared to controls. (B) Correlation analysis of human-mouse-matched miRNA expression between human controls and $\mathrm{C} 3 \mathrm{H}$ sham mice, showing a Pearson correlation coefficient of $r=0.81$. (C) miR-499-3p is strongly downregulated in human $\mathrm{VM}$ biopsies compared to controls. ${ }^{*} \mathrm{P}<0.05$. 
a
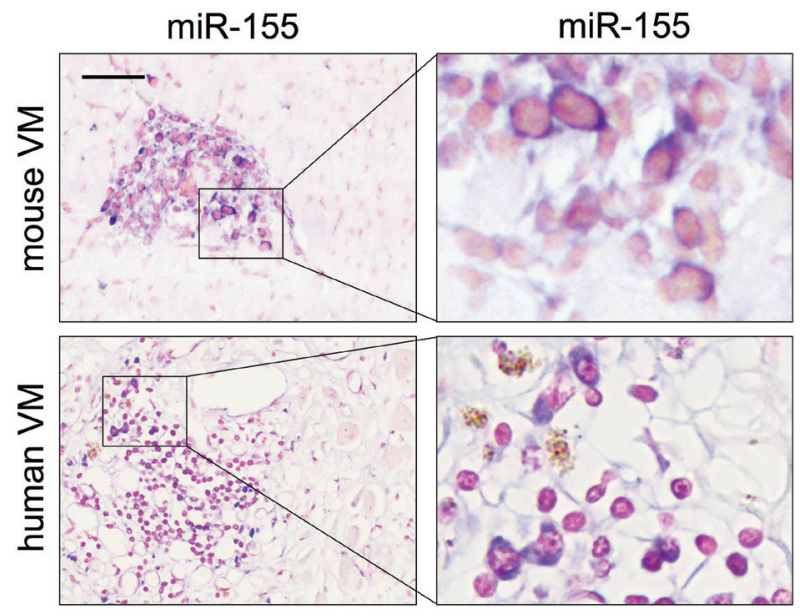

C control

stimulated

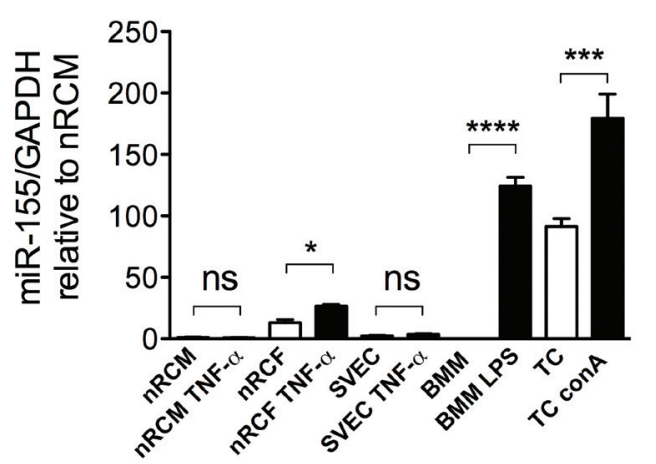

Supplementary Figure 6. MiR-155 is predominantly expressed by inflammatory cells in the mouse heart during viral myocarditis. (A) In situ hybridization of miR-155 (blue) on left ventricular sections of mouse VM ( $\mathrm{C} 3 \mathrm{H} 7$ days). A triangular-shaped inflammatory patch stains dark blue after hybridization with a miR-155 detection probe while virtually no miR-155 is detected in adjacent normal myocardium. Only pink nuclear counterstaining is observed when using an LNA control probe. The inset shows perinuclear localization of miR-155 in inflammatory cells. (B) In situ hybridization for miR-155 in left ventricular biopsies of a human VM patient, again showing localization of miR-155 specifically in inflammatory cells in inflamed regions but not in adjacent myocardium. (C) MiR-155 expression in a cell panel of rat neonatal myocytes $(\mathrm{RCM})$, fibroblasts ( $\mathrm{RCCF}$ ), adult mouse endothelial cells (SVEC), primary bone marrow-derived macrophages (BMM) and splenic T cells (TC), stimulated for 24 hours with pro-inflammatory stimuli (TNF- $\alpha$ for $n R C M$, nRCM and SVEC; LPS for BMM; Con A for TC). Expression levels are presented as relative to nRCM. Scale bars: $100 \mu \mathrm{m}$. ${ }^{*} \mathrm{P}<0.05,{ }^{*} \mathrm{P}<0.01,{ }^{*}{ }^{*} \mathrm{P}<0.001,{ }^{*}{ }^{*} \mathrm{P}<0.0001$. 
a

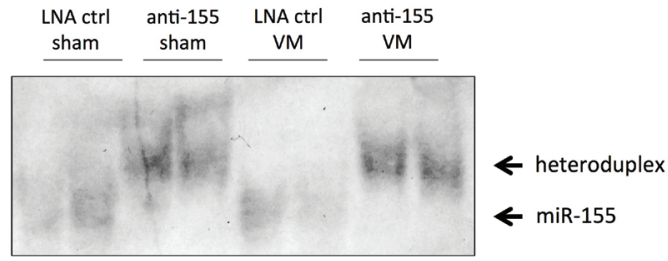

C LNA control VM vs anti-155 VM vs anti-155 sham

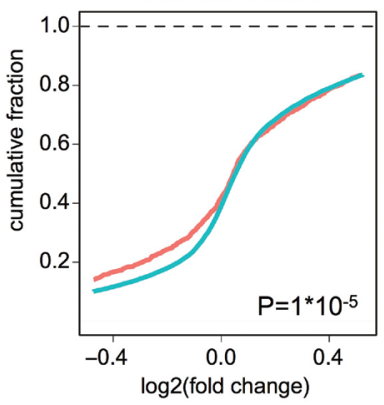

b

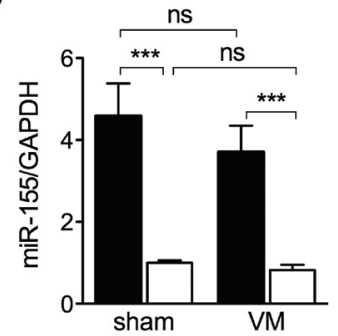

Supplementary Figure 7. Inhibition of miR-155 by LNA-antimiR-155 treatment. (A) Native Northern blot of spleen samples showing stable duplex formation between the LNA-antimiR and mature miR155, as evidenced by the band shift in both sham and VM animals. (B) qRT-PCR confirmed significant suppression of miR-155 in mouse spleens by LNA-antimiR-155 ( $n=6$ per sham groups and $n=11$ per VM group). (C) De-repression of mRNAs with predicted 7-mer miR-155 seed sites in sham versus VM animals treated with LNA-antimiR-155 or LNA control. Cumulative distributions of mRNA changes in VM versus sham conditions demonstrate suppression of miR-155 targets upon up-regulation of miR-155 during VM (one-sided Kolmogorov-Smirnov test; control VM versus control sham: $p=1.0^{*} 10^{-5}$; antimiR-155 VM versus antimiR-155 sham: $\left.p=1.4{ }^{*} 10^{-3}\right) .{ }^{* *} \mathrm{P}<0.001$. 
a
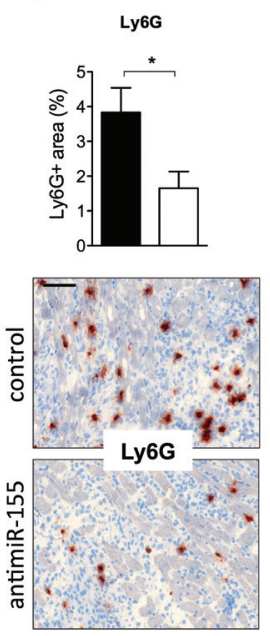

b
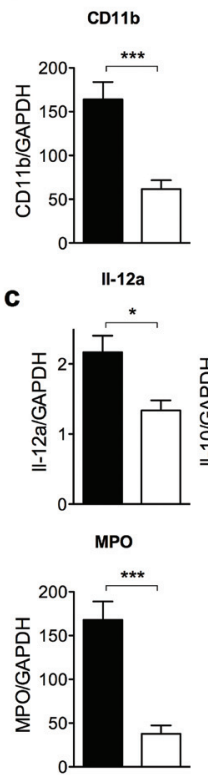

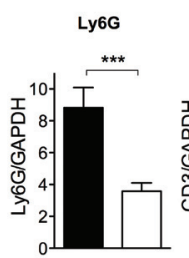

||-10

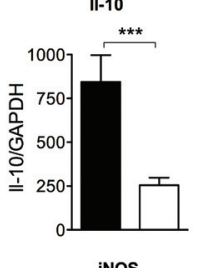

iNOS

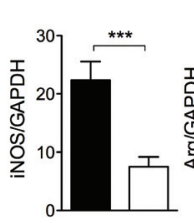

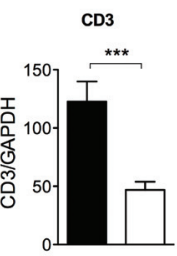

||-17
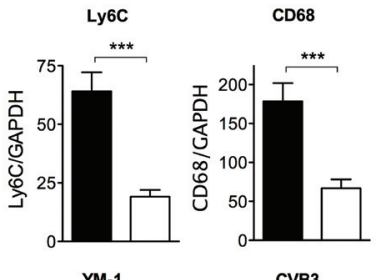

CVB3
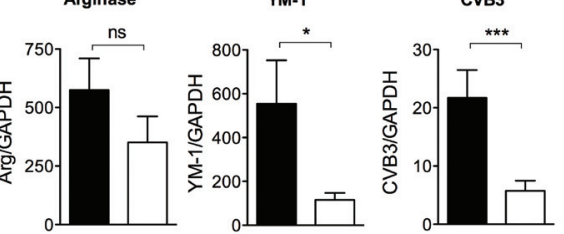

Supplementary Figure 8. Inhibition of miR-155 suppresses inflammatory signaling in VM at day 7 after infection. MiR-155 inhibition reduced cardiac infiltration of Ly6G-immunoreactive neutrophil granulocytes (A) (scale bars: $100 \mu \mathrm{m})$. (B) qRT-PCR measurements showing that miR-155 inhibition suppressed the cardiac expression of CD11b (macrophages), Ly6G (neutrophils) and CD3 (T lymphocytes) in VM. (C) A wide range of pro- and anti-inflammatory transcripts is suppressed during VM after treatment with LNA-antimiR-155. ( $n=6$ per sham group and $n=11$ per VM group). Cytokine levels are shown as relative to sham. ${ }^{*} P<0.05$, ${ }^{*} P<0.01,{ }^{* *} P<0.001$.
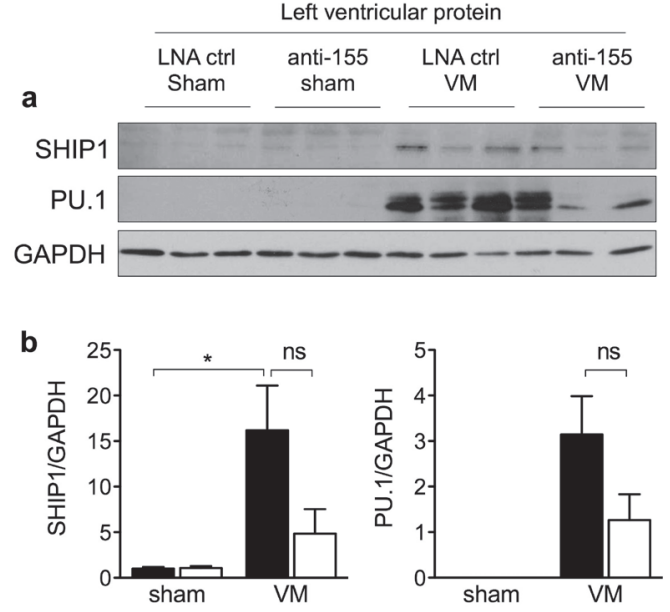

Supplementary Figure 9. Cardiac protein levels of direct miR-155 targets reflect intensity of inflammation. Western blots (A) and protein level quantifications (B) of the direct miR-155 targets PU.1 and SHIP1 on left ventricular protein homogenates. Both proteins were hardly expressed in sham hearts and more abundant during viral myocarditis. Protein levels of PU.1 and SHIP1 were not de-repressed by miR-155 inhibition, but were higher in control VM hearts, which contained more infiltrating inflammatory cells ( $n=3$ per group). ${ }^{*} P<0.05$. 
LNA control VM $\square$ LNA-antimiR-155 VM

a
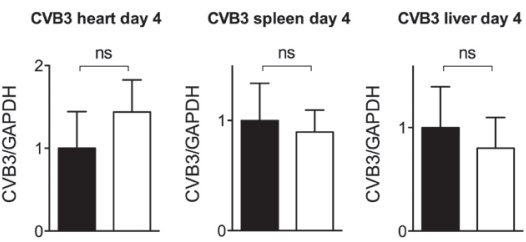

b
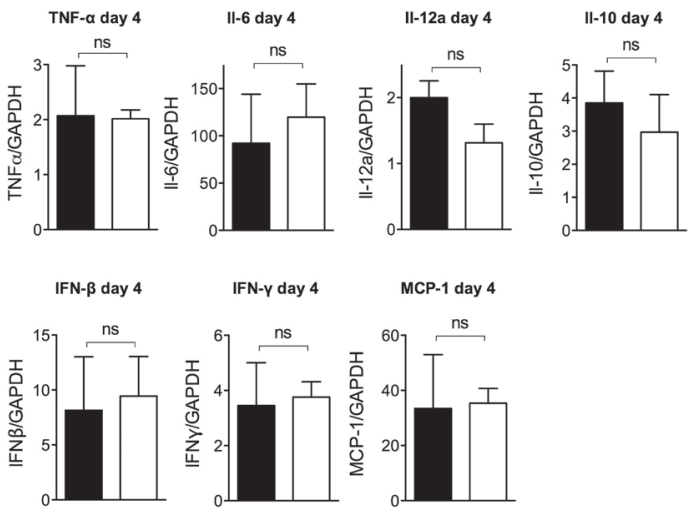

\section{Supplementary Figure 10.} miR-155 inhibition does not affect cardiac viral load and cytokine expression at day 4 after infection.

(A) qRT-PCR measurements of CVB3 levels in the heart, spleen and liver are not affected by miR-155 inhibition at day 4 , before infiltration of cardiac inflammatory cells. (B) Expression of TNF- $\alpha$, II-6, II-12a, II-10, IFN- $\beta$, IFN- $\gamma$, and the chemokine macrophage chemo-attractant protein-1 (MCP-1) (relative to sham) by cardiac resident cells is unchanged by miR-155 inhibition at day 4 ( $n=6$ per group).
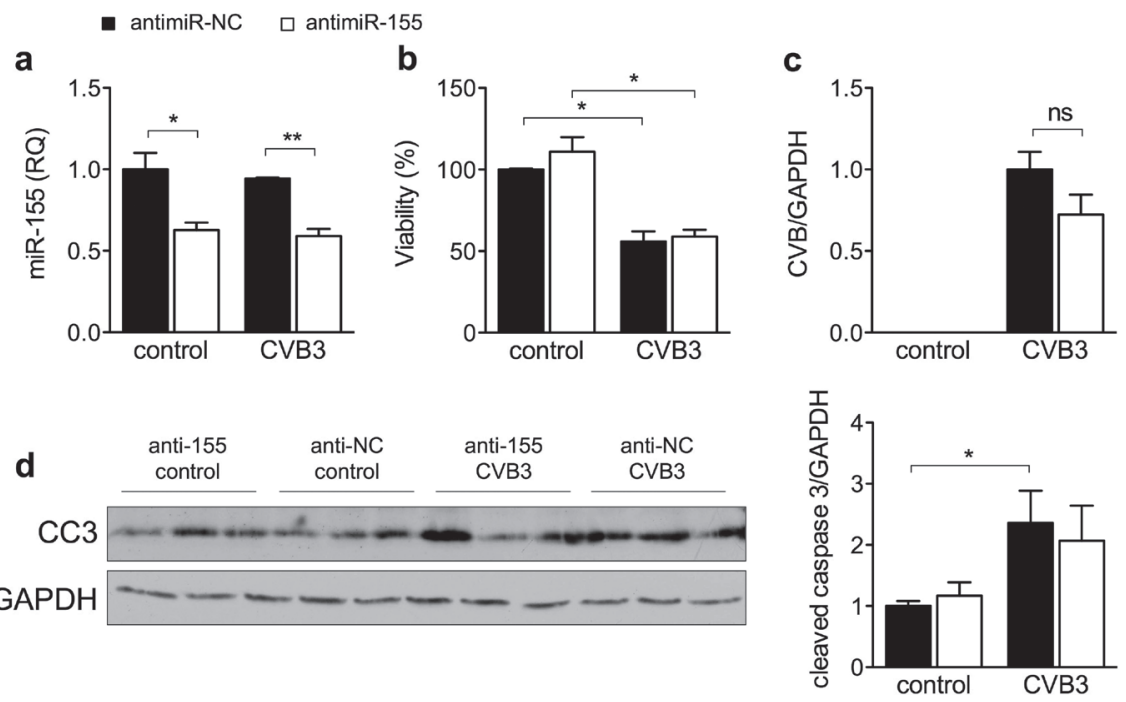

Supplementary Figure 11. Mir-155 inhibition does not affect myocyte viability after CVB3 infection. To investigate whether the low levels of miR-155 expressed by cardiomyocytes influence myocyte viability after CVB3 infection, we knocked down miR-155 in vitro in neonatal rat cardiomyocytes (nRCM) (A) before infecting with $\mathrm{CVB} 3$ at $\mathrm{MOI}=1$. (B) Viability was similarly decreased in antimiR-155 or control nRCM at 120 hourse after infection (B) and the number of viral genome copies was not different between treatment groups (C). (D) Western blotting for cleaved caspase 3 (CC3) as a marker of apoptosis revealed that cleaved capsase 3 increases were not affected by miR-155 inhibition ( $n=3$ per group). ${ }^{*} P<0.05,{ }^{*} P<0.01$. 


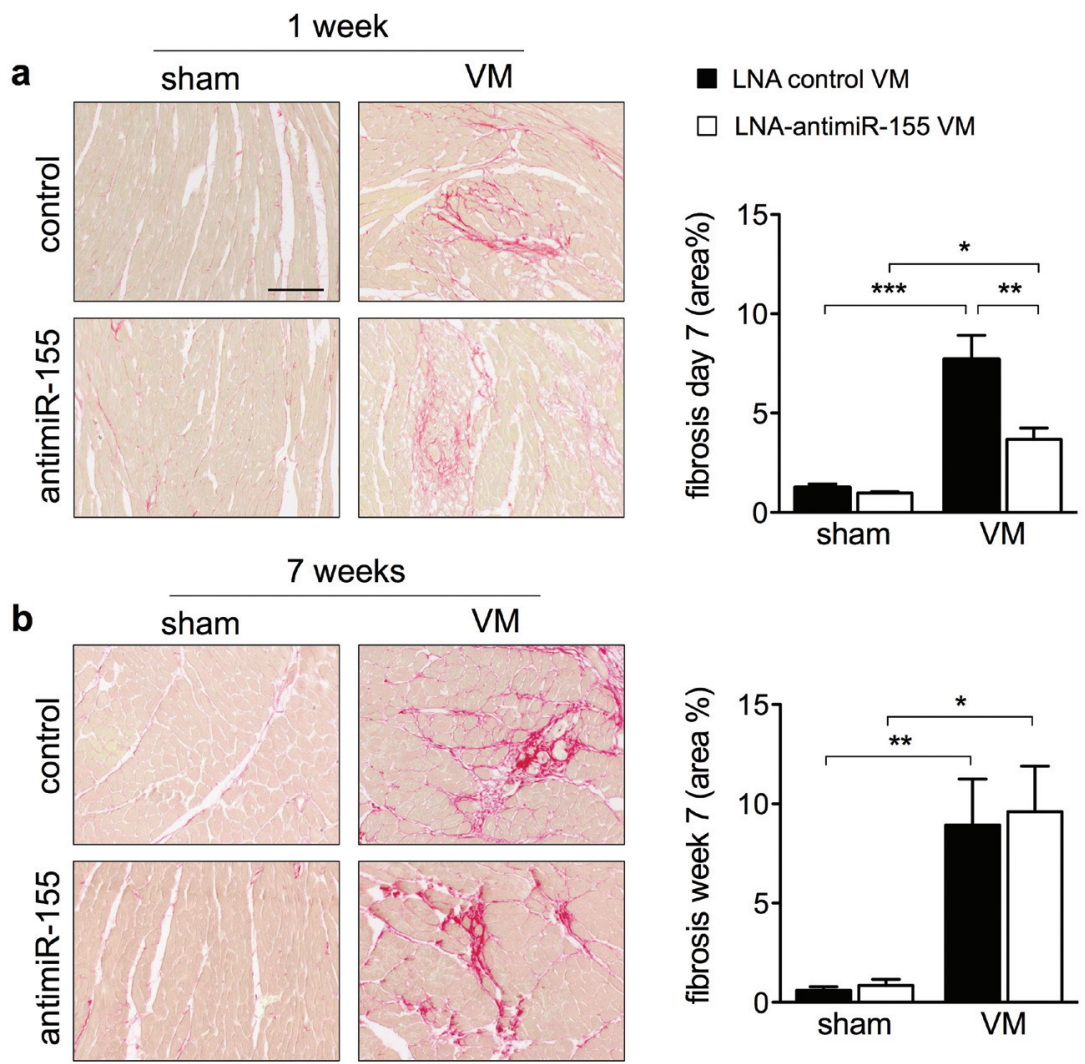

Supplementary Figure 12. Cardiac fibrosis at $\mathbf{1}$ and $\mathbf{7}$ weeks of viral myocarditis. Cardiac collagen deposition as measured by sirius red staining was reduced by miR-155 inhibition at 1 week (A), but was not different between groups after 7 weeks if viral myocarditis (B). ( 1 week: $n=6$ per sham group and $n=11$ per VM group; 7 weeks: $n=6$ per sham group, $n=14$ for LNA control VM and $n=19$ for LNA-antimiR-155 VM). ${ }^{*} P$ $<0.05,{ }^{*} P<0.01,{ }^{*} * P<0.001$. 


\section{Supplementary Methods}

\section{Animal studies}

All mouse experiments were performed according to the guidelines for care and use of laboratory animals approved by the institutional animal care committee. For viral myocarditis (VM) experiments, 4-week-old male susceptible C3H mice or resistant N-strain C57BI/6 mice $^{1}$ (Harlan, Boxmeer, The Netherlands) were inoculated intraperitoneally with $2 \times 10^{5}$ plaque forming units of Coxsackievirus B3 (CVB3) or saline. To investigate cardiac microRNA expression longitudinally during $\mathrm{VM}$, animals were euthanized and dissected for further molecular and histological analysis at days 0,4 and $7(n=3$ animals per strain per time point).

For microRNA knockdown in vivo, a 15-mer LNA-antimiR oligonucleotide targeting mmumiR-155 or an LNA control was administered to male 4-week-old C3H mice intravenously at a dose of $25 \mathrm{mg} / \mathrm{kg}$ per injection at days 1, 4 and 6 post-CVB3 infection. Group sizes were at least $n=6$ for sham, $n=6$ for $V M$ groups at day 4 and $n=11$ for $V M$ groups at day 7. Chronic treatment for the 7-week-follow-up included 2 weeks of high dose treatment (25 mg/kg/injection iv) followed by 5 weeks of low dose treatment ( $5 \mathrm{mg} / \mathrm{kg} /$ injection ip). Group sizes were at least $n=6$ for sham and $n=11$ for VM groups in the 1 -week analysis, $n=6$ per sham and $n=8$ for VM for flow cytometry, and $n=6$ for sham and $n=20$ for $V M$ in the 7-week analysis. Echocardiography was performed with the Vevo770 (Visualsonics, Toronto, Canada) and probe RMV707B (15-45 MHz) at days 14 and 49. Organs were snapfrozen or fixed for 4-6 hours in 1\% paraformaldehyde for histology. Circulating leukocyte numbers were counted in fresh heparinized mouse blood samples using the Abbott (Waver, Belgium) Cell-Dyn 3700. Uptake of fluorescently labeled LNA-antimiRs in circulating leukocytes was evaluated using the FACS Canto II flow cytometer (Becton Dickenson, San Diego, CA) after removing erythrocytes by hypotonic lysis with ammonium chloride.

\section{Patient material}

All human material was obtained during routine sampling used for clinical purposes, stored in a coded way and available for research purposes in accordance with the Declaration of Helsinki and the ethical committee at Maastricht University Medical Center. For microRNA profiling, right ventricular septal biopsies were used, obtained during routine clinical sampling from patients with acute myocarditis $(n=4)$. Patients with a definite clinical history of myocarditis were included ${ }^{2}$ (Supplementary Table 1). Patients presented with an episode of upper airway or gastro-intestinal infection, followed after 1-3 weeks by chest pain. All myocarditis patients had elevated troponin T plasma levels and immunohistochemically proven cardiac inflammation ( $\mathrm{n}>14 \mathrm{CD} 45$ positive cells $/ \mathrm{mm}^{2}$ ). In all patients, virus presence was demonstrated in the cardiac biopsies (Supplementary Table 1). Coronary artery disease was excluded either by CAG or CT. Controls $(n=6)$ consisted of age-matched patients with unexplained ventricular tachy-arrhythmias but with a normal ejection fraction and the absence of systemic or cardiac inflammation or virus presence at the time of biopsy (Supplementary Table 1). Cardiac biopsies were immediately snap-frozen for RNA analysis or formalin-fixed for histological examination. 


\section{In situ hybridization}

In situ hybridization was performed as described previously $y^{3,4}: 4 \mu \mathrm{m}$ sections were prepared from $48 \mathrm{hr} 10 \%$ neutral buffered formalin fixed human or mouse cardiac biopsies. Sections were de-waxed, dehydrated, air-dried briefly and incubated in $1.3 \mathrm{mg} / \mathrm{ml}$ Pepsin/0.1 mM $\mathrm{HCl}$ solution for $5 \mathrm{~min}$ at $37^{\circ} \mathrm{C}$. After a brief wash in DEPC MQ, sections were incubated overnight at $42.5^{\circ} \mathrm{C}$ in $1 \times$ hybridisation buffer (ENZO Life sciences, Farmingdal, NY, \#33808) with either 100 nM mmu-miR-155 DIG labeled probe (EXIQON, Vedbaek, Denmark, \#3947101) or a scrambled control probe (\#99004-01). The following day the sections were washed once in 5xSSC and twice in 0.2xSSC at hybridisation temperature. Subsequently, sections were blocked in DIG blocking buffer from the DIG Wash and Block Buffer Set (Roche, Woerden, The Netherlands, \#11585762001) for 20 min followed by a 45 min incubation in 1:500 anti-DIG-AP, Fab fragments (Roche, \#11093274910). Sections were washed 3x5 min with TBS and incubated 3 min in DIG detection buffer at room temperature. AP signal was detected by using NBT/BCIP tablets (Roche cat.no.11697471001) for 4 hours at $30^{\circ} \mathrm{C}$ in darkness. Finally, sections were counterstained with fast red (Sigma, Zwijndrecht, The Netherlands) briefly washed in $\mathrm{MQ}$, quickly dehydrated through an ethanol gradient and mounted with entellan (EMS, Hatfield, PA \#14800).

Double stain for ISH/IHC was conducted as follows: For ISH, slides were dewaxed, dehydrated and briefly washed in PBS. Prot.K/PBS solution 15ug/ml (Ambion, \#AM2546) was applied for $10 \mathrm{~min}$. at $37^{\circ} \mathrm{C}$. Slides were washed twice in PBS for $5 \mathrm{~min}$., dehydrated and air dried for $15 \mathrm{~min}$. Subsequently slides were treated as described above. For the CD3 $\mathrm{IHC}$ the ISH slides were treated with $1.3 \mathrm{mg} / \mathrm{ml}$ Pepsin in $0.1 \mathrm{mM} \mathrm{HCl}$ for $8 \mathrm{~min}$ at $37^{\circ} \mathrm{C}$ followed with 2x5min PBS washes. Next, slides were blocked in 1\%BSA/TBS and 10\% goat serum. Slides were then incubated with primary CD3 antibody (Neomarkers, Fremont, CA, USA, \#RM-9107-s) for $16 \mathrm{hrs}$ at $4^{\circ} \mathrm{C}$. Next slides were incubated with secondary

Supplementary Table 1. Acute viral myocarditis and control sample donor characteristics

\begin{tabular}{|c|c|c|c|}
\hline Characteristic & $\begin{array}{l}\text { Viral Myocarditis } \\
\qquad(n=4)\end{array}$ & $\begin{array}{c}\text { Controls } \\
(n=6)\end{array}$ & $P^{*}$ \\
\hline Age, years & $39 \pm 20$ & $38 \pm 11$ & 0.96 \\
\hline Women & $0(0)$ & 2 (33.3) & 0.52 \\
\hline \multicolumn{4}{|l|}{ Echocardiography } \\
\hline $\operatorname{LVEF}(\%)$ & $25 \pm 15$ & $55 \pm 7$ & 0.002 \\
\hline $\mathrm{EDD}(\mathrm{mm})$ & $48 \pm 10$ & $56 \pm 5$ & 0.15 \\
\hline PVB19 > threshold $†$ & $4(100)$ & $0(0)$ & 0.005 \\
\hline PVB19 titer (c/ug DNA) & $1855 \pm 1508$ & $79 \pm 56$ & 0.04 \\
\hline CD45 cell/mm² & $80 \pm 39$ & $11 \pm 4$ & 0.005 \\
\hline Serum Troponin T, $\mu \mathrm{g} / \mathrm{L}$ & $2.9 \pm 3.7$ & ND & \\
\hline
\end{tabular}

Data are presented as mean $\pm \mathrm{SD}$, or $\mathrm{n}(\%)$

* P-values based on student t-test, Mann-Whitney test or Chi-square analysis, $†$ Threshold value: 500 copies/mg cardiac DNA, LVEF Left Ventricular Ejection, EDD End diastolic Diameter, ND Not determined 
PolyHRP antibody (Epitomics, Burlingame, CA, USA \#3051-1) for $16 \mathrm{hrs}$ at $4^{\circ} \mathrm{C}$, washed 3 times with TBST and developed with DAB. No counterstain was applied; slides were dehydrated and mounted in entallan (EMS, Hatfield, PA, USA \#14800).

\section{RNA isolation, microRNA arrays, Northern blotting and quantitative RT-PCR}

RNA was isolated from left ventricular mouse tissue samples and right ventricular septal biopsies using the miRVANA isolation kit (Ambion, Warrington, UK), according to the manufacturer's instructions and without enrichment for small RNAs. Potential genomic DNA contamination was removed using the DNA-free kit (Ambion).

For Northern blot analysis total RNA was loaded on non-denaturing gels to show heteroduplex formation between the LNA-antimiR and mature miR-155. Briefly, 1-5 $\mu \mathrm{g}$ RNA per sample was loaded without pre-heating on a non-denaturing $20 \%$ acrylamide gel and ran for 2 hrs at $100 \mathrm{~V}$, transferred to a nylon membrane (Roche, Mannheim, Germany) using a semi-dry blotting device (Biorad) for 15 minutes at 20V. Blots were hybridized at $37^{\circ} \mathrm{C}$ with dual DIG-labeled LNA probes (Exiqon, Vedbaek, Denmark) and visualized using the DIG Wash and Block buffer set (Roche) and CDP-star reagent (Roche).

For quantitative real-time PCR analysis, cDNA was generated using the miScript kit (Qiagen, Venlo, The Netherlands) and quantified using IQ SYBR-green supermix (Bio-Rad, Veenendal, The Netherlands) in a MylQ iCycler (Bio-Rad). LNA-based primer assays (Exiqon) were used for detection of mature microRNAs. A complete list of primer sequences is provided in Supplementary Table 2.

Supplementary Table 2. Primer sequences

\begin{tabular}{|c|c|c|}
\hline Gene & Forward primer & Reverse primer \\
\hline CVB3 & ACGAATCCCAGTGTGTTTTGG & TGCTCAAAAACGGTATGGACAT \\
\hline mmu-Arginase & CAAGACAGGGCTCCTTTCAG & GCTTATGGTTACCCTCCCGT \\
\hline $\mathrm{mmu}-\mathrm{CD} 3 \varepsilon$ & TATCACTCTGGGCTTGCTGA & TAGTCTGGGTTGGGAACAGG \\
\hline mmu-CD11b & GGCAGGAGTCGTATGTGAGG & CAGCAGTGATGAGAGCCAAG \\
\hline mmu-CD68 & CCAGCTGTTCACCTTGACCT & CAATGATGAGAGGCAGCAAG \\
\hline mmu-GAPDH & GGTGGACCTCATGGCCTACA & СTCTCTTGCTCAGTGTCCTTGCT \\
\hline mmu-IFN- $\gamma$ & GCGTCATTGAATCACACCTG & CTGGACCTGTGGGTTGTTG \\
\hline mmu-IFN- $\beta 1$ & AGCACTGGGTGGAATGAGAC & TCCCACGTCAATCTTTCCTC \\
\hline mmu-interleukin-6 & CAAAGCCAGAGTCCTTCAGAG & GCCACTCCTTCTGTGACTCC \\
\hline mmu-interleukin-10 & ATCGATTTCTCCCCTGTGAA & TGGCCTTGTAGACACCTTGG \\
\hline mmu-interleukin-12a & CTAGACAAGGGCATGCTGGT & TCTCCCACAGGAGGTTTCTG \\
\hline mmu-interleukin-17 & TCCAGAAGGCCCTCAGACTA & TGAGCTTCCCAGATCACAGA \\
\hline mmu-iNOS & GCAGCGGCTCCATGACTCCC & AGGTGGTCCTCCTCCGGGTG \\
\hline mmu-Ly6C & TCTTGTGGCCCTACTGTGTG & TGAGCAATGCAGAATCCATC \\
\hline mmu-Ly6G & GATGGATTTTGCGTTGCTCT & GTCCAGAGTAGTGGGGCAGA \\
\hline mmu-MCP-1 & GTTGGCTCAGCCAGATGCA & AGCCTACTCATTGGGATCATCTTG \\
\hline mmu-MPO & GCCTCATTGGCACTCAGTTT & GTGGTGATGCCAGTGTTGTC \\
\hline mmu-TNF- $\alpha$ & CCACCACGCTCTTCTGTCTA & AGGGTCTGGGCCATAGAACT \\
\hline mmu-YM-1 & TAAGACTGGAATTGGTGCCC & CAGACCTCAGTGGCTCCTTC \\
\hline rno-GAPDH & GGTGGACCTCATGGCCTACA & СTCTCTTGCTCTCAGTATCCTTGCT \\
\hline
\end{tabular}




\section{MicroRNA and Messenger RNA expression array analysis}

Total RNA from human biopsies was hybridized to Illumina Human miRNAv2 Expression Panel arrays by ServiceXS (Leiden, The Netherlands). These arrays contained 858 human microRNA sequences described in Sanger's Institute miRBase release 17, plus novel content derived using Illumina's sequencing technologies, making up a total of 1.146 assays per chip. Expression values were quantile normalized using the beadarray ${ }^{5}$ package in $\mathrm{R}$. The limma package ${ }^{6}$ was used to identify differentially regulated microRNAs between experimental groups. MicroRNAs were considered differentially expressed with a $p$-Value of $<0.05$. Raw data are available in the online Data Supplement.

Total RNA from mouse left ventricles was hybridized to $\mu$ Paraflo ${ }^{\circledR}$ arrays (LC Sciences, Houston, TX) based on miRBase Sequence Database Release 12.0 (Sept 2008) containing 627 mouse microRNAs. In-depth data analysis was performed by LC Sciences. Similarity of mouse and human microRNA was determined by a global sequence alignment between the mature sequences as provided by miRBase. MicroRNAs were considered the same with a maximum of 1 mismatch or 2 gap extension to equalize their length.

RNA from hearts of LNA control- and LNA-antimiR-155-treated mice (sham and 7 days post CVB3 injection) was hybridized to Affymetrix GeneChip Mouse Gene 1.0 ST arrays (ArrayExpress accession: E-MEXP-3395). The chip quality was assessed using the affyQCReport in R. All chips passed the quality control. Expression values were normalized between chips using $r m a^{7}$ and the CustomCDF ${ }^{8}$ provided by the University of Michigan. The limma package was used to identify differentially regulated mRNAs (Benjamini \& Hochberg adjusted p-Value < 0.05). De-repression of cardiac mRNAs with canonical 7-mer miR-155 seed match sites (Ensembl, February 2011) after LNA-antimiR treatment was evaluated using cumulative distribution functions and significance of the differences from mRNAs with no sites was determined using one-sided Kolmogorov-Smirnov tests. Unsupervised hierarchical clustering was based on Euclidean distance and Ward's clustering method.

\section{Design and synthesis of LNA oligonucleotides}

The LNA-antimiRs were synthesized as unconjugated oligonucleotides with a complete phosphorothioate backbone. The perfectly matching LNA-antimiR-155 oligonucleotide 5'TcAcAATtaG ${ }_{m}$ CAtTA $-3^{\prime}$ was complementary to nucleotides 2-16 in the mature murine miR-155 sequence while the LNA-control oligonucleotide was synthesized with the following sequence: 5'- TcAta ${ }_{m}$ CTatAtGa $_{m}$ CA -3' (uppercase: LNA; lowercase: DNA; mC denotes LNA methylcytosine).

\section{Histology}

Paraffin-embedded $4 \mu \mathrm{m}$ LV sections were stained with hematoxylin-eosin (HE) or Picrosirius red (Sigma). Left ventricular cryosections of $7 \mu \mathrm{m}$ were acetone-fixed and immunohistochemically stained with monoclonal rat-anti-mouse antibodies against CD11b (R\&D systems, Minneapolis, MN, \#MAB11124), Ly6G (BD Pharmingen, Breda The Netherlands, \#551459) or a polyclonal rat anti-mouse antibody against CD3 (gift from Dr. Menno de Winther). Photomicrographs were obtained (Leitz DMRXE; Leica, Wetzlar, 
Germany) and immunoreactivity was quantified as the number of positive cells per $\mathrm{mm}^{2}$, or when too many cells overlapped as stained area percentage, using OWin morphometry software (Leica).

For serial staining of leukocyte markers (Mac3, CD3 and CD45) on tissue fixed with 10\% neutral buffered formalin for in situ hybridization, the following protocol was used. Slides were dewaxed and dehydrated through an Ethanol gradient and incubated in PBS. Next HIER was preformed in a pressure cooker with $10 \mathrm{mM}$ Citric Acid, 0,05\% tween20, pH6,0 for $5 \mathrm{~min}$. Slides were cooled down and incubated in PBS. Slides were then blocked in $1 \%$ BSA/TBS with the appropriate normal serum for one hour. Next, slides were incubated in 1\%BSA/TBS with antibody diluted 1:300 (CD45, BD \#55039/ CD3, Neomarkers, \#RM9107-s/ MAC3, BD \#550292) overnight at $4^{\circ} \mathrm{C}$. Slides were washed 3×5min. in TBST pH8.4 and incubated in antibody solution 1\%BSA/TBST with 1:500 of the appropriate antibody (Sigma; Goat-anti-Rat \#A8438, Goat anti Rabbit \#A3937). Subsequently slides were washed 3x5min. in TBST pH 8.4 and developed in NBT/BCIP (Roche cat.no.11697471001). Slides were counterstained in fast red (Sigma, Zwijndrecht, The Netherlands), dehydrated and mounted in entallan (EMS, Hatfield, PA \#14800).

\section{Western blotting}

Protein extracts were obtained from left ventricular tissue, from the cardiac immune fraction, or from nRCMs, and were resolved by SDS-PAGE and immunoblotted to a PVDF membrane. Blots were incubated with antibodies against PU.1 (9G7 Cell Signaling Technology, Danvers, MA \#2258), and SHIP1 (D-1163 Cell Signaling Technology, \#2728). GAPDH (Fitzgerald, Acton, MA, RDI-TRK5G4-6C5) was used as a loading control.

\section{Cardiac fractionation and flow cytometry}

Animals were euthanized and the whole heart flushed with PBS by injecting a syringe in the left ventricle cavity and nicking the right atria. The heart was dissected out, the atria removed and placed in PBS, for isolation of the immune cell fraction. Briefly, the ventricles were minced into $1 \mathrm{~mm}$ pieces with a sterile surgical blade, and placed in an enzymatic solution $(125 \mathrm{U} / \mathrm{ml}$ collagenase type $\mathrm{XI}, 60 \mathrm{U} / \mathrm{ml}$ hyaluronidase type I-s, $60 \mathrm{U} / \mathrm{ml}$ DNase1, and $450 \mathrm{U} / \mathrm{ml}$ collagenase type I, Sigma-Aldrich) for $1 \mathrm{hr}$ at $37^{\circ} \mathrm{C}$. After trituration, the single cell suspension was underlayed with Histopaque solution, in order to separate the immune from the non-immune fraction. The interphase (immune fraction) was rinsed with PBS and cell pellets were either lysed for further molecular analysis, or leukocyte analysis by FACS analysis. Leukocytes subpopulations were defined as follows; Regulatory T lymphocytes, (CD45+, CD3+, CD4+, CD25+, Foxp3+) Naïve T-helper lymphocytes (CD45+, CD3+, CD4+, CD62 L+), Effector T-helper lymphocytes (CD45+, CD3+, CD4+, CD62L-), Naive Cytotoxic lymphocytes (CD45+, CD3+, CD8+, CD62L+), Effector Cytotoxic T lymphocytes (CD45+, CD3+, CD8+, CD62L-), B lymphocytes (CD45+, B220+), Granulocytes (CD45+, CD11b+ Ly6G+), Monocytes (CD45+, CD11b+ F4/80-), Pro-inflammatory monocytes (CD45+, CD11b+F4/80-, Ly6C+), Macrophages (CD45+, CD11b+F4/80+), with the following antibodies anti-CD45 (Biolegend clone 30-F11), CD62L (e-bioscience, clone mel-14), F4/80 (Biolegend, clone BM8), CD3 (Ebioscience, clone 145-C11), CD45R/B220 (Ebioscience, 
clone RA3-6B2), Ly6G (BD, clone 1A8) or CD11b (BD, clone M1/70), Ly6C (Miltenyi, $1 \mathrm{G} 7$. G10), CD4 (BD, clone RM4-5), CD8 (BD, clone 53-6.7) and CD25 (Ebiosciencs, clone PC61.5), FoxP3 (regulatory T cells) (Ebiosciences, clone FJK-16s).

\section{In vitro experiments}

Neonatal rat ventricular cardiac myocytes (RCMs) and fibroblasts (RCFs) were isolated by enzymatic disassociation of 1-to-2-day-old pups, plated on gelatinized 6-well plates and cultured as previously described ${ }^{9}$. Per well, $10^{6}$ RCMs or RCFs were grown on high serum media for 48 hours. They were then put on low serum media overnight before stimulation with 10 ng/mL TNF- $\alpha$ (cat. \#315-01A, PeproTech, Rocky Hill, NJ, USA) for 24 hours. Experiments with murine endothelial cells (SVEC) were performed under similar conditions in 12-well plates, seeding $2.5^{*} 10^{5}$ cells per well. Bone marrow-derived macrophages (BMMs) were harvested from the femurs and tibias of adult $\mathrm{C} 3 \mathrm{H}$ mice and placed in $\mathrm{R} 10$ (RPMI 1640 medium, containing 10\% heat-inactivated fetal calf serum, $100 \mathrm{U} / \mathrm{ml}$ penicillin, $100 \mu \mathrm{g} / \mathrm{ml}$ streptomycin, $2 \mathrm{mM}$ L-glutamine, and $10 \mathrm{mM}$ Hepes) supplemented with $15 \%$ L-929 cell-conditioned medium. After 7 days, cells were scraped and seeded in 6-wells at $10^{6}$ cells per well. After incubation overnight in low serum media, BMMs were stimulated with 10ng/mL lipopolysaccharide (from E. coli 055.B5; Sigma) for 24 hours. Primary T cells were isolated from adult mouse spleens by mechanical dissociation, followed by magnetic bead separation of CD4/8 positive cells by negative selection using the MACS Pan T cell isolation kit (Miltenyi Biotec, Auburn, CA, USA, \#130-095-130). Cells were seeded in 6-wells at $10^{6}$ cells per 6-well and stimulated with $5 \mu \mathrm{g} / \mathrm{mL}$ concanavalin A (Sigma, \#C5275) for 24 hours. After stimulation RNA was isolated with miRVana (Ambion) and quantitative PCR was performed as described above.

To assess the effect of miR-155 inhibition in vitro on $\mathrm{nRCM}$ viability $10^{6} \mathrm{RCMs}$ per well in 6-well plates were grown on high serum media for 24 hours before transfection using lipofectamine 2000 (Invitrogen, Breda, The Netherlands, \#11668-019) according to manufacturer's instructions. Cells were transfected with miRVANA inhibitors against mmumiR-155 (Ambion, \#4464084-MH13058) or negative control (Ambion \#4464076) at 40nM. After 6 hours, medium was replaced and cells were put on low serum media overnight before infection with CVB3 at $\mathrm{MOI}=1$. After 72 hours, cells were harvested for protein extraction, or for RNA isolation as described above. Alternatively, after 120 hours, cells were washed twice with PBS following lysis using the CellTiter-Glo Limunscent Cell Viability Assay (Promega, Madison, WI, G7570) and read on a Spectramax L luminescent plate reader (Molecular Devices, Sunny Ville, CA). 
Supplementary Table 3a. C57BI/6N: 4 days versus sham

\begin{tabular}{|c|c|c|c|}
\hline microRNA & $P$ value & FC (log2) & FC \\
\hline mmu-miR-483* & 0,044702563 & $-1,082$ & 0,472 \\
\hline mmu-miR-503* & 0,015530864 & $-0,940$ & 0,521 \\
\hline mmu-miR-654-5p & 0,03707061 & $-0,925$ & 0,527 \\
\hline mmu-miR-494 & 0,048975676 & $-0,478$ & 0,718 \\
\hline mmu-miR-151-5p & 0,030107263 & $-0,302$ & 0,811 \\
\hline mmu-miR-17* & 0,017454267 & 0,407 & 1,326 \\
\hline mmu-miR-411 & 0,034315214 & 0,578 & 1,493 \\
\hline mmu-miR-135a* & 0,007063276 & 0,810 & 1,753 \\
\hline mmu-miR-300 & 0,042411588 & 0,918 & 1,890 \\
\hline mmu-miR-140 & 0,048201516 & 1,007 & 2,009 \\
\hline mmu-miR-93* & 0,032048773 & 1,149 & 2,217 \\
\hline
\end{tabular}

Supplementary Table 3b. C57BI/6N: 4 days versus sham

\begin{tabular}{lccc}
\hline microRNA & P value & FC (log2) & FC \\
\hline mmu-miR-541 & 0,02439305 & $-1,209$ & 0,433 \\
mmu-miR-151-5p & 0,006003813 & $-0,751$ & 0,594 \\
mmu-miR-486 & 0,043727804 & $-0,729$ & 0,603 \\
mmu-miR-503* & 0,03201153 & $-0,727$ & 0,604 \\
mmu-miR-320 & 0,025917845 & $-0,629$ & 0,647 \\
mmu-miR-351 & 0,042124193 & $-0,626$ & 0,648 \\
mmu-miR-361 & 0,006356742 & $-0,485$ & 0,714 \\
mmu-miR-378 & 0,03034244 & $-0,425$ & 0,745 \\
mmu-miR-324-5p & 0,047891583 & $-0,216$ & 0,861 \\
mmu-miR-323-5p & 0,021281615 & 0,308 & 1,238 \\
mmu-miR-139-3p & 0,029451575 & 0,334 & 1,260 \\
mmu-miR-17* & 0,019301327 & 0,507 & 1,422 \\
mmu-miR-718 & 0,00689129 & 0,975 & 1,966 \\
mmu-miR-93* & 0,014088051 & 1,072 & 2,102 \\
mmu-miR-223 & 0,034406003 & 1,453 & 2,738 \\
mmu-miR-106a & 0,013237868 & 1,624 & 3,083 \\
mmu-miR-146b & 0,01227604 & 1,759 & 3,384 \\
mmu-miR-20b & 0,03763973 & 1,943 & 3,846 \\
mmu-miR-208b & 0,001702103 & 2,613 & 6,118 \\
\hline
\end{tabular}

Supplementary Table 3c. C3H: 4 days versus sham

\begin{tabular}{lccc}
\hline microRNA & P value & FC (log2) & FC \\
\hline mmu-miR-329 & 0,009322313 & $-0,723$ & 0,606 \\
mmu-miR-322* & 0,045099653 & $-0,610$ & 0,655 \\
mmu-miR-370 & 0,020455325 & $-0,608$ & 0,656 \\
mmu-miR-361 & 0,035907492 & $-0,218$ & 0,860 \\
mmu-miR-324-3p & 0,048320077 & 0,214 & 1,160 \\
mmu-miR-181a-1* & 0,002824315 & 0,386 & 1,307 \\
mmu-miR-34b-3p & 0,049852878 & 0,421 & 1,339 \\
mmu-miR-615-5p & 0,020096587 & 0,470 & 1,385 \\
mmu-miR-374 & 0,02763182 & 0,509 & 1,423 \\
mmu-miR-362-5p & 0,02583122 & 0,647 & 1,566 \\
mmu-miR-365 & 0,03683327 & 0,660 & 1,580 \\
mmu-miR-467f & 0,048136983 & 1,115 & 2,166 \\
mmu-miR-223 & 0,04803933 & 1,236 & 2,356 \\
mmu-miR-146b & 0,019650105 & 1,267 & 2,407 \\
mmu-miR-21 & 0,024212485 & 1,295 & 2,454 \\
\hline
\end{tabular}


Supplementary table $3 \mathrm{~d}$. C3H: 7 days versus sham

\begin{tabular}{|c|c|c|c|}
\hline microRNA & $P$ value & FC (log2) & FC \\
\hline mmu-miR-30e* & 0,046358544 & $-2,351$ & 0,196 \\
\hline mmu-miR-149 & 0,03397871 & $-2,174$ & 0,222 \\
\hline mmu-miR-30a* & 0,038407456 & $-2,094$ & 0,234 \\
\hline mmu-miR-378* & 0,026823249 & $-1,989$ & 0,252 \\
\hline mmu-miR-181d & 0,034686834 & $-1,977$ & 0,254 \\
\hline mmu-miR-322* & 0,004189921 & $-1,942$ & 0,260 \\
\hline mmu-miR-486 & 0,032215957 & $-1,813$ & 0,285 \\
\hline mmu-miR-181b & 0,002808145 & $-1,781$ & 0,291 \\
\hline mmu-miR-192 & 0,046755955 & $-1,676$ & 0,313 \\
\hline mmu-miR-499 & 0,005804902 & $-1,426$ & 0,372 \\
\hline mmu-miR-208a & 0,049824037 & $-1,225$ & 0,428 \\
\hline mmu-miR-574-3p & 0,000201125 & $-1,184$ & 0,440 \\
\hline mmu-miR-151-3p & 0,011075961 & $-1,140$ & 0,454 \\
\hline mmu-miR-128 & 0,02740018 & $-1,101$ & 0,466 \\
\hline mmu-miR-125a-5p & 0,038804565 & $-1,059$ & 0,480 \\
\hline mmu-miR-676 & 0,00871934 & $-1,008$ & 0,497 \\
\hline mmu-miR-145 & 0,019773467 & $-0,972$ & 0,510 \\
\hline mmu-miR-185 & 0,013118264 & $-0,941$ & 0,521 \\
\hline mmu-miR-329 & 0,019983388 & $-0,880$ & 0,543 \\
\hline mmu-miR-151-5p & 0,011855478 & $-0,868$ & 0,548 \\
\hline mmu-miR-351 & 0,003871412 & $-0,864$ & 0,550 \\
\hline mmu-miR-181a & 0,014240269 & $-0,845$ & 0,557 \\
\hline mmu-miR-22* & 0,020532861 & $-0,842$ & 0,558 \\
\hline mmu-miR-99b & 0,03776402 & $-0,780$ & 0,582 \\
\hline mmu-miR-24-2* & 0,035912655 & $-0,761$ & 0,590 \\
\hline mmu-miR-148a & 0,035202067 & $-0,726$ & 0,605 \\
\hline mmu-miR-455 & 0,019717637 & $-0,688$ & 0,621 \\
\hline mmu-miR-361 & 0,016715042 & $-0,677$ & 0,625 \\
\hline mmu-miR-320 & 0,03896415 & $-0,654$ & 0,635 \\
\hline mmu-miR-195 & 0,03590963 & $-0,566$ & 0,676 \\
\hline mmu-miR-107 & 0,0321193 & $-0,460$ & 0,727 \\
\hline mmu-miR-744 & 0,045502525 & $-0,431$ & 0,742 \\
\hline mmu-miR-103 & 0,04819953 & $-0,348$ & 0,786 \\
\hline mmu-miR-143 & 0,023859914 & $-0,253$ & 0,839 \\
\hline mmu-let-7a & 0,030434748 & 0,178 & 1,131 \\
\hline mmu-miR-1 & 0,013920028 & 0,285 & 1,218 \\
\hline mmu-miR-126-3p & 0,04826773 & 0,328 & 1,255 \\
\hline mmu-miR-23b & 0,020723164 & 0,421 & 1,339 \\
\hline mmu-miR-106b & 0,021067401 & 0,422 & 1,340 \\
\hline mmu-miR-23a & 0,04349021 & 0,442 & 1,359 \\
\hline mmu-miR-16 & 0,02403476 & 0,568 & 1,483 \\
\hline mmu-miR-466f & 0,038378604 & 0,598 & 1,513 \\
\hline mmu-miR-19b & 0,026014028 & 0,657 & 1,577 \\
\hline mmu-miR-350 & 0,04972223 & 0,752 & 1,684 \\
\hline mmu-miR-17* & 0,011749918 & 0,789 & 1,728 \\
\hline mmu-miR-15a & 0,039716665 & 0,798 & 1,738 \\
\hline mmu-miR-532-5p & 0,034992076 & 0,867 & 1,824 \\
\hline mmu-miR-15b & 0,013798017 & 0,875 & 1,834 \\
\hline mmu-miR-425 & 0,013770207 & 0,888 & 1,851 \\
\hline mmu-miR-362-5p & 0,002680239 & 0,923 & 1,897 \\
\hline mmu-miR-674 & 0,019204611 & 0,929 & 1,904 \\
\hline mmu-miR-210 & 0,018491874 & 0,988 & 1,983 \\
\hline
\end{tabular}


follow up of Supplementary table 3d

\begin{tabular}{llll}
\hline microRNA & P value & FC (log2) & FC \\
\hline mmu-miR-34a & 0,01819858 & 1,013 & 2,018 \\
mmu-miR-20a & 0,024550011 & 1,081 & 2,116 \\
mmu-miR-17 & 0,00604084 & 1,115 & 2,166 \\
mmu-miR-146a & 0,00706058 & 1,133 & 2,193 \\
mmu-miR-494 & 0,04678588 & 1,211 & 2,315 \\
mmu-miR-340-5p & 0,037993435 & 1,466 & 2,762 \\
mmu-miR-106a & 0,001500131 & 1,518 & 2,863 \\
mmu-miR-501-3p & 0,016947836 & 1,578 & 2,985 \\
mmu-miR-705 & 0,042335194 & 1,583 & 2,996 \\
mmu-miR-1187 & 0,041873924 & 1,643 & 3,122 \\
mmu-miR-714 & 0,03181346 & 1,883 & 3,689 \\
mmu-miR-20b & 0,02066468 & 1,903 & 3,741 \\
mmu-miR-1224 & 0,024642378 & 1,954 & 3,874 \\
mmu-miR-18a & 0,009808551 & 2,156 & 4,456 \\
mmu-miR-1892 & 0,032332353 & 2,302 & 4,931 \\
mmu-miR-130b & 0,006447384 & 2,377 & 5,193 \\
mmu-miR-342-3p & 0,011159668 & 2,596 & 6,046 \\
mmu-miR-142-5p & 0,036101244 & 2,838 & 7,149 \\
mmu-miR-21 & 0,000807731 & 3,676 & 12,783 \\
mmu-miR-155 & 0,010672883 & 3,906 & 14,986 \\
mmu-miR-223 & 0,001946641 & 4,821 & 28,268 \\
mmu-miR-146b & 0,008104529 & 5,633 & 49,618 \\
\hline
\end{tabular}


Supplementary table $4 \mathrm{a}$. $\mathrm{C} 57 \mathrm{BI} / 6 \mathrm{~N}$ versus $\mathrm{C} 3 \mathrm{H}$ : sham

\begin{tabular}{lccc}
\hline microRNA & P value & FC (log2) & FC \\
\hline mmu-miR-342-3p & 0,023943413 & $-2,240$ & 0,212 \\
mmu-miR-491 & 0,035962764 & $-1,089$ & 0,470 \\
mmu-miR-805 & 0,032383937 & $-0,995$ & 0,502 \\
mmu-miR-503* & 0,04833348 & $-0,874$ & 0,546 \\
mmu-miR-654-5p & 0,034900457 & $-0,871$ & 0,547 \\
mmu-miR-434-3p & 0,038859706 & $-0,774$ & 0,585 \\
mmu-miR-540-5p & 0,002989688 & $-0,497$ & 0,708 \\
mmu-miR-29a & 0,027615903 & $-0,451$ & 0,732 \\
mmu-miR-27b & 0,020436365 & 0,319 & 1,247 \\
mmu-miR-107 & 0,04304382 & 0,361 & 1,285 \\
mmu-miR-135a* & 0,048545305 & 0,393 & 1,313 \\
mmu-miR-181a & 0,02844735 & 0,489 & 1,404 \\
mmu-miR-1899 & 0,046357077 & 0,615 & 1,531 \\
mmu-miR-361 & 0,001403497 & 0,744 & 1,675 \\
mmu-miR-140 & 0,032061037 & 1,137 & 2,200 \\
mmu-miR-28* & 0,031555235 & 1,383 & 2,609 \\
mmu-miR-547 & 0,017727492 & 1,587 & 3,004 \\
mmu-miR-208b & 0,035234578 & 1,975 & 3,931 \\
mmu-miR-582-5p & 0,001915029 & 2,821 & 7,068 \\
\hline
\end{tabular}

Supplementary table $4 \mathrm{~b}$. C57BI/6N versus $\mathrm{C} 3 \mathrm{H}:$ 4d

\begin{tabular}{|c|c|c|c|}
\hline microRNA & $P$ value & FC (log2) & FC \\
\hline mmu-miR-342-3p & 0,004602955 & $-2,416$ & 0,187 \\
\hline mmu-miR-204 & 0,045218993 & $-1,553$ & 0,341 \\
\hline mmu-miR-155 & 0,036248315 & $-1,099$ & 0,467 \\
\hline mmu-miR-34a & 0,04637811 & $-1,051$ & 0,483 \\
\hline mmu-miR-805 & 0,001562989 & $-1,001$ & 0,500 \\
\hline mmu-miR-30c-1 * & 0,009433536 & $-0,846$ & 0,556 \\
\hline mmu-miR-30a* & 0,01951384 & $-0,771$ & 0,586 \\
\hline mmu-miR-22* & 0,0436794 & $-0,650$ & 0,637 \\
\hline mmu-miR-146a & 0,008665415 & $-0,632$ & 0,645 \\
\hline mmu-miR-135a* & 0,02008814 & $-0,609$ & 0,656 \\
\hline mmu-miR-29c & 0,035149448 & $-0,577$ & 0,671 \\
\hline mmu-miR-150 & 0,008604468 & $-0,529$ & 0,693 \\
\hline mmu-miR-29a & 0,009543358 & $-0,445$ & 0,734 \\
\hline mmu-miR-128 & 0,02925138 & $-0,318$ & 0,802 \\
\hline mmu-miR-214 & 0,025768809 & 0,477 & 1,392 \\
\hline mmu-miR-207 & 0,038149375 & 0,481 & 1,396 \\
\hline mmu-miR-148a & 0,012546075 & 0,487 & 1,401 \\
\hline mmu-miR-199a-3p & 0,013693704 & 0,531 & 1,445 \\
\hline mmu-miR-499 & 0,039355725 & 0,684 & 1,606 \\
\hline mmu-miR-181b & 0,032301154 & 0,690 & 1,613 \\
\hline mmu-miR-181a-1 * & 0,04342271 & 0,795 & 1,735 \\
\hline mmu-miR-361 & 0,012509491 & 0,850 & 1,803 \\
\hline mmu-miR-34b-3p & 0,019788396 & 0,855 & 1,809 \\
\hline mmu-miR-28 & 0,005523557 & 1,161 & 2,237 \\
\hline mmu-miR-669f & 0,03222844 & 1,344 & 2,539 \\
\hline mmu-miR-181c & 0,006812654 & 1,378 & 2,600 \\
\hline mmu-miR-28* & 0,02789194 & 1,397 & 2,634 \\
\hline mmu-miR-181d & 0,000814127 & 1,508 & 2,845 \\
\hline mmu-miR-547 & 0,03526841 & 1,723 & 3,301 \\
\hline mmu-miR-335-5p & 0,015183391 & 2,964 & 7,805 \\
\hline
\end{tabular}


Supplementary table 4c. C57BI/6N versus $\mathrm{C} 3 \mathrm{H}$ : $7 \mathrm{~d}$

\begin{tabular}{|c|c|c|c|}
\hline microRNA & P value & FC $(\log 2)$ & FC \\
\hline mmu-miR-30a* & 0,02593803 & $-2,804$ & 0,143 \\
\hline mmu-miR-30 * & 0,02780424 & $-2,426$ & 0,186 \\
\hline mmu-miR-98 & 0,048142307 & $-1,924$ & 0,263 \\
\hline mmu-miR-805 & 0,037200842 & $-1,879$ & 0,272 \\
\hline mmu-miR-145* & 0,02535808 & $-1,767$ & 0,294 \\
\hline mmu-miR-208a & 0,03536968 & $-1,727$ & 0,302 \\
\hline mmu-miR-188-5p & 0,04030971 & $-1,672$ & 0,314 \\
\hline mmu-miR-192 & 0,039125722 & $-1,618$ & 0,326 \\
\hline mmu-miR-204 & 0,03919689 & $-1,609$ & 0,328 \\
\hline mmu-miR-30c-2* & 0,007318904 & $-1,499$ & 0,354 \\
\hline mmu-miR-342-5p & 0,027309835 & $-1,437$ & 0,369 \\
\hline mmu-miR-101b & 0,013836786 & $-1,381$ & 0,384 \\
\hline mmu-miR-22* & 0,018138826 & $-1,207$ & 0,433 \\
\hline mmu-miR-128 & 0,027744314 & $-1,134$ & 0,456 \\
\hline mmu-miR-30b* & 0,02581216 & $-1,006$ & 0,498 \\
\hline mmu-miR-872 & 0,04112682 & $-0,932$ & 0,524 \\
\hline mmu-miR-504 & 0,015230159 & $-0,930$ & 0,525 \\
\hline mmu-miR-574-3p & 0,014991037 & $-0,833$ & 0,561 \\
\hline mmu-miR-615-3p & 0,04504323 & $-0,809$ & 0,571 \\
\hline mmu-miR-24-2* & 0,034350313 & $-0,755$ & 0,593 \\
\hline mmu-miR-125a-5p & 0,048810005 & $-0,748$ & 0,595 \\
\hline mmu-miR-328 & 0,04480886 & $-0,747$ & 0,596 \\
\hline mmu-miR-322* & 0,008333288 & $-0,700$ & 0,615 \\
\hline mmu-miR-26b & 0,036034495 & $-0,677$ & 0,625 \\
\hline mmu-miR-30d & 0,0431715 & $-0,645$ & 0,640 \\
\hline mmu-miR-196a* & 0,012290002 & $-0,532$ & 0,691 \\
\hline mmu-miR-365 & 0,019036058 & $-0,493$ & 0,711 \\
\hline mmu-miR-151-3p & 0,049959578 & $-0,409$ & 0,753 \\
\hline mmu-miR-30c & 0,02388088 & $-0,337$ & 0,792 \\
\hline mmu-miR-29a & 0,001371107 & $-0,286$ & 0,820 \\
\hline mmu-miR-30b & 0,010157838 & $-0,253$ & 0,839 \\
\hline mmu-miR-27a & 0,019476408 & 0,199 & 1,148 \\
\hline mmu-miR-27b & 0,005049864 & 0,219 & 1,164 \\
\hline mmu-miR-191 & 0,03516194 & 0,306 & 1,237 \\
\hline mmu-miR-24 & 0,012635543 & 0,365 & 1,288 \\
\hline mmu-miR-23a & 0,040018834 & 0,437 & 1,354 \\
\hline mmu-miR-23b & 0,035773236 & 0,440 & 1,356 \\
\hline mmu-miR-106b & 0,024372695 & 0,479 & 1,394 \\
\hline mmu-miR-361 & 0,009872661 & 0,552 & 1,466 \\
\hline mmu-miR-16 & 0,03980788 & 0,581 & 1,496 \\
\hline mmu-miR-690 & 0,017558878 & 0,649 & 1,568 \\
\hline mmu-miR-324-5p & 0,046218343 & 0,665 & 1,586 \\
\hline mmu-miR-19b & 0,047728945 & 0,687 & 1,610 \\
\hline mmu-miR-28 & 0,03340208 & 0,694 & 1,617 \\
\hline mmu-miR-199a-3p & 0,011984071 & 0,799 & 1,740 \\
\hline mmu-miR-20a & 0,019996602 & 0,849 & 1,802 \\
\hline mmu-miR-17 & 0,02529782 & 0,864 & 1,819 \\
\hline mmu-miR-28* & 0,029774155 & 0,976 & 1,966 \\
\hline mmu-miR-425 & 0,009870599 & 0,982 & 1,976 \\
\hline mmu-miR-140* & 0,04188976 & 0,997 & 1,996 \\
\hline mmu-miR-106a & 0,030994477 & 1,005 & 2,006 \\
\hline mmu-miR-214 & 0,04392994 & 1,015 & 2,021 \\
\hline
\end{tabular}


follow up of Supplementary table $4 \mathrm{C}$

\begin{tabular}{lccc}
\hline microRNA & P value & FC (log2) & FC \\
\hline mmu-miR-709 & 0,040391672 & 1,030 & 2,042 \\
mmu-miR-532-5p & 0,007309648 & 1,116 & 2,167 \\
mmu-miR-501-3p & 0,04015988 & 1,140 & 2,204 \\
mmu-miR-541 & 0,01138485 & 1,218 & 2,326 \\
mmu-miR-376b & 0,019579086 & 1,233 & 2,350 \\
mmu-miR-210 & 0,003312363 & 1,284 & 2,435 \\
mmu-miR-21 & 0,024541995 & 1,508 & 2,844 \\
mmu-miR-184 & 0,03810928 & 1,535 & 2,898 \\
mmu-miR-18a & 0,005404913 & 1,739 & 3,338 \\
mmu-miR-130b & 0,006619324 & 2,079 & 4,226 \\
mmu-miR-669a & 0,026402878 & 2,265 & 4,807 \\
mmu-miR-142-5p & 0,043886542 & 2,794 & 6,935 \\
mmu-miR-155 & 0,007051461 & 2,923 & 7,582 \\
mmu-miR-223 & 0,008762343 & 3,024 & 8,135 \\
mmu-miR-146b & 0,014882721 & 4,237 & 18,850 \\
\hline
\end{tabular}

Supplementary table 5 . Human VM vs control

\begin{tabular}{|c|c|c|c|}
\hline microRNA & P value & FC $(\log 2)$ & FC \\
\hline hsa-miR-499-3p & 0,01771 & $-1,653$ & 0,318 \\
\hline hsa-miR-302b & 0,01824 & $-1,464$ & 0,362 \\
\hline hsa-miR-490-3p & 0,00819 & $-1,436$ & 0,370 \\
\hline hsa-miR-455-5p & 0,00755 & $-1,347$ & 0,393 \\
\hline hsa-miR-140-5p & 0,02908 & $-1,324$ & 0,399 \\
\hline hsa-miR-497 & 0,02668 & $-1,323$ & 0,400 \\
\hline hsa-miR-361-3p & 0,00029 & $-1,214$ & 0,431 \\
\hline hsa-miR-106a & 0,00139 & $-1,184$ & 0,440 \\
\hline hsa-miR-20b & 0,01424 & $-1,121$ & 0,460 \\
\hline hsa-miR-302a & 0,01833 & $-1,108$ & 0,464 \\
\hline hsa-miR-652 & 0,00206 & $-1,096$ & 0,468 \\
\hline hsa-miR-186 & 0,04768 & $-1,087$ & 0,471 \\
\hline hsa-miR-133b & 0,03597 & $-1,061$ & 0,479 \\
\hline hsa-miR-1296 & 0,01056 & $-1,002$ & 0,499 \\
\hline hsa-miR-221* & 0,0052 & $-0,994$ & 0,502 \\
\hline hsa-miR-193b & 0,0398 & $-0,962$ & 0,513 \\
\hline hsa-miR-9 & 0,0445 & $-0,956$ & 0,515 \\
\hline hsa-miR-584 & 0,01509 & $-0,955$ & 0,516 \\
\hline hsa-miR-302a* & 0,04563 & $-0,954$ & 0,516 \\
\hline hsa-miR-1271 & 0,01369 & $-0,906$ & 0,534 \\
\hline hsa-miR-99b & 0,00388 & $-0,904$ & 0,534 \\
\hline hsa-miR-324-5p & 0,00806 & $-0,883$ & 0,542 \\
\hline hsa-miR-107 & 0,04434 & $-0,871$ & 0,547 \\
\hline hsa-miR-30c-2* & 0,01439 & $-0,871$ & 0,547 \\
\hline hsa-miR-27b* & 0,02178 & $-0,86$ & 0,551 \\
\hline hsa-miR-30e & 0,00866 & $-0,854$ & 0,553 \\
\hline hsa-miR-149 & 0,02979 & $-0,823$ & 0,565 \\
\hline hsa-miR-651 & 0,0138 & $-0,803$ & 0,573 \\
\hline hsa-miR-29c* & 0,0153 & $-0,765$ & 0,588 \\
\hline hsa-miR-483-3p & 0,01664 & $-0,751$ & 0,594 \\
\hline hsa-miR-1237 & 0,01511 & $-0,697$ & 0,617 \\
\hline hsa-miR-30a* & 0,00307 & $-0,683$ & 0,623 \\
\hline
\end{tabular}


follow up of Supplementary table 5

\begin{tabular}{|c|c|c|c|}
\hline microRNA & $P$ value & FC (log2) & FC \\
\hline hsa-miR-93 & 0,00006 & $-0,683$ & 0,623 \\
\hline hsa-miR-103a & 0,01541 & $-0,669$ & 0,629 \\
\hline hsa-miR-147b & 0,00409 & $-0,65$ & 0,637 \\
\hline hsa-miR-24-1* & 0,01616 & $-0,584$ & 0,667 \\
\hline hsa-miR-190 & 0,03847 & $-0,575$ & 0,671 \\
\hline hsa-miR-181a* & 0,03631 & $-0,57$ & 0,674 \\
\hline hsa-miR-545 & 0,00744 & $-0,559$ & 0,679 \\
\hline hsa-miR-30a & 0,01196 & $-0,498$ & 0,708 \\
\hline hsa-miR-1287 & 0,0397 & $-0,493$ & 0,711 \\
\hline hsa-miR-133a & 0,00702 & $-0,437$ & 0,739 \\
\hline hsa-miR-548b-3p & 0,00115 & $-0,391$ & 0,763 \\
\hline hsa-miR-182* & 0,00314 & $-0,381$ & 0,768 \\
\hline hsa-miR-1233 & 0,03365 & $-0,36$ & 0,779 \\
\hline hsa-miR-30c & 0,00565 & $-0,359$ & 0,780 \\
\hline hsa-miR-30b & 0,02737 & $-0,333$ & 0,794 \\
\hline hsa-miR-921 & 0,00857 & $-0,329$ & 0,796 \\
\hline hsa-miR-554 & 0,03872 & $-0,317$ & 0,803 \\
\hline hsa-miR-1267 & 0,04009 & $-0,296$ & 0,815 \\
\hline hsa-miR-885-5p & 0,03958 & $-0,291$ & 0,817 \\
\hline hsa-miR-30b* & 0,02491 & $-0,285$ & 0,821 \\
\hline hsa-miR-27b & 0,03414 & $-0,225$ & 0,856 \\
\hline hsa-miR-24 & 0,04245 & $-0,221$ & 0,858 \\
\hline hsa-miR-30e* & 0,02487 & $-0,149$ & 0,902 \\
\hline hsa-miR-1266 & 0,00986 & $-0,127$ & 0,916 \\
\hline hsa-miR-147 & 0,02309 & $-0,123$ & 0,918 \\
\hline hsa-miR-1827 & 0,04579 & $-0,102$ & 0,932 \\
\hline hsa-miR-146a* & 0,01923 & 0,127 & 1,092 \\
\hline hsa-miR-1243 & 0,03945 & 0,134 & 1,097 \\
\hline hsa-miR-1253 & 0,02298 & 0,147 & 1,107 \\
\hline hsa-miR-552 & 0,01275 & 0,178 & 1,131 \\
\hline hsa-miR-202 & 0,0106 & 0,193 & 1,143 \\
\hline hsa-miR-323b-5p & 0,00263 & 0,204 & 1,152 \\
\hline hsa-miR-155* & 0,00218 & 0,22 & 1,165 \\
\hline hsa-miR-21 & 0,04875 & 0,228 & 1,171 \\
\hline hsa-miR-619 & 0,04981 & 0,252 & 1,191 \\
\hline hsa-miR-624 & 0,00783 & 0,271 & 1,207 \\
\hline hsa-miR-633 & 0,02698 & 0,326 & 1,254 \\
\hline hsa-miR-580 & 0,02838 & 0,349 & 1,274 \\
\hline hsa-miR-564 & 0,01807 & 0,362 & 1,285 \\
\hline hsa-miR-645 & 0,02141 & 0,368 & 1,291 \\
\hline hsa-miR-488 & 0,04594 & 0,379 & 1,300 \\
\hline hsa-miR-1263 & 0,01048 & 0,4 & 1,320 \\
\hline hsa-miR-1224-5p & 0,02474 & 0,401 & 1,320 \\
\hline hsa-miR-217 & 0,03155 & 0,406 & 1,325 \\
\hline hsa-miR-452* & 0,00034 & 0,423 & 1,341 \\
\hline hsa-miR-597 & 0,0391 & 0,467 & 1,382 \\
\hline hsa-miR-92b* & 0,04421 & 0,471 & 1,386 \\
\hline hsa-miR-20b* & 0,00053 & 0,485 & 1,400 \\
\hline hsa-miR-563 & 0,01002 & 0,511 & 1,425 \\
\hline hsa-miR-382 & 0,03068 & 0,521 & 1,435 \\
\hline hsa-miR-493* & 0,04296 & 0,546 & 1,460 \\
\hline hsa-miR-501-3p & 0,01185 & 0,556 & 1,470 \\
\hline hsa-miR-299-3p & 0,00038 & 0,578 & 1,493 \\
\hline
\end{tabular}


follow up of Supplementary table 5

\begin{tabular}{lccc}
\hline microRNA & P value & FC (log2) & FC \\
\hline hsa-miR-450b-3p & 0,00956 & 0,592 & 1,507 \\
hsa-miR-488* & 0,01036 & 0,597 & 1,513 \\
hsa-miR-132 & 0,0032 & 0,688 & 1,611 \\
hsa-miR-302d* & 0,02976 & 0,694 & 1,618 \\
hsa-miR-629 & 0,00337 & 0,72 & 1,647 \\
hsa-miR-892b & 0,00783 & 0,741 & 1,671 \\
hsa-miR-891a & 0,00815 & 0,744 & 1,675 \\
hsa-miR-631 & 0,03682 & 0,758 & 1,691 \\
hsa-miR-558 & 0,01039 & 0,763 & 1,697 \\
hsa-miR-1246 & 0,01835 & 0,883 & 1,844 \\
hsa-miR-216a & 0,03411 & 0,923 & 1,896 \\
hsa-miR-432 & 0,03504 & 0,944 & 1,924 \\
hsa-miR-92b & 0,00738 & 0,995 & 1,993 \\
hsa-miR-212 & 0,00231 & 1,002 & 2,003 \\
hsa-miR-409-5p & 0,00009 & 1,057 & 2,081 \\
hsa-miR-889 & 0,0007 & 1,454 & 2,740 \\
hsa-miR-155 & 0,00008 & 1,816 & 3,521 \\
hsa-miR-511 & 0,00001 & 2,402 & 5,285 \\
hsa-miR-146b-5p & 0,0095 & 3,573 & 11,901 \\
\hline
\end{tabular}

Bold microRNAs are significantly regulated in the same direction in murine VM (C3H mice day 7)

Supplementary Table 6. Biometrics of viral myocarditis mice treated with LNA-antimiR-155 or antimiRcontrol

\begin{tabular}{|c|c|c|c|c|c|c|c|c|}
\hline & \multicolumn{4}{|c|}{ Day 7} & \multicolumn{4}{|c|}{ Day 50} \\
\hline & $\begin{array}{l}\text { LNA control } \\
\text { sham }\end{array}$ & $\begin{array}{l}\text { LNA-155 } \\
\text { sham }\end{array}$ & $\begin{array}{c}\text { LNA control } \\
\text { VM }\end{array}$ & $\begin{array}{l}\text { LNA-155 } \\
\text { VM }\end{array}$ & $\begin{array}{c}\text { LNA control } \\
\text { sham }\end{array}$ & $\begin{array}{l}\text { LNA-155 } \\
\text { sham }\end{array}$ & $\begin{array}{c}\text { LNA control } \\
\text { VM }\end{array}$ & $\begin{array}{l}\text { LNA-155 } \\
\text { VM }\end{array}$ \\
\hline Number & 6 & 6 & 12 & 11 & 6 & 6 & 14 & 19 \\
\hline $\begin{array}{l}\text { Body weight } \\
\text { baseline (g) }\end{array}$ & $21.4 \pm 0.6$ & $19.5 \pm 0.4$ & $20.3 \pm 0.5$ & $21.3 \pm 0.3$ & $17.1 \pm 1.4$ & $18.0 \pm 0.3$ & $17.7 \pm 0.9$ & $18.0 \pm 0.8$ \\
\hline $\begin{array}{l}\text { Body weight } \\
\text { at sacrifice (g) }\end{array}$ & $24.1 \pm 0.5$ & $23.1 \pm 0.5$ & $\begin{array}{l}20.5 \pm \\
0.8+t \dagger\end{array}$ & $23.2 \pm 0.5^{* *}$ & $30.2 \pm 0.8$ & $30.3 \pm 0.5$ & $28.2 \pm 0.5 \dagger$ & $29.2 \pm 0.6$ \\
\hline $\begin{array}{l}\text { Heart weight / } \\
\text { BW (mg/g) }\end{array}$ & $3.9 \pm 0.1$ & $3.9 \pm 0.1$ & $5.7 \pm 0.2$ †十† & $4.8 \pm 0.3 t^{*}$ & $5.4 \pm 0.1$ & $5.6 \pm 0.1$ & $6.5 \pm 0.2 \dagger$ & $6.3 \pm 0.2$ \\
\hline $\begin{array}{l}\text { Lung weight / } \\
\text { BW (mg/g) }\end{array}$ & $6.9 \pm 0.3$ & $6.7 \pm 0.2$ & $7.2 \pm 0.3$ & $6.6 \pm 0.1$ & $8.6 \pm 0.2$ & $8.3 \pm 0.1$ & $9.7 \pm 0.5$ & $9.4 \pm 0.4$ \\
\hline $\begin{array}{l}\text { Liver weight / } \\
\text { BW (mg/g) }\end{array}$ & $66.1 \pm 2.2$ & $71.4 \pm 1.7$ & $56.7 \pm 4.0$ & $63.7 \pm 1.9 \dagger$ & $77.0 \pm 2.6$ & $80.5 \pm 2.2$ & $82.2 \pm 1.7$ & $81.7 \pm 1.6$ \\
\hline $\begin{array}{l}\text { Kidney weight / } \\
\text { BW (mg/g) }\end{array}$ & $8.7 \pm 0.5$ & $8.4 \pm 0.3$ & $7.1 \pm 0.4 \dagger$ & $9.1 \pm 0.1 *$ & $11.9 \pm 0.4$ & $12.7 \pm 0.3$ & $12.0 \pm 0.3$ & $11.9 \pm 0.3$ \\
\hline $\begin{array}{l}\text { Spleen weight / } \\
\text { BW (mg/g) }\end{array}$ & $4.6 \pm 0.5$ & $4.3 \pm 0.2$ & $7.9 \pm 0.5+\dagger$ & $7.1 \pm 0.4 \dagger \dagger$ & $4.4 \pm 0.1$ & $4.1 \pm 0.1$ & $4.8 \pm 0.2$ & $5.0 \pm 0.2 *$ \\
\hline Fibrotic area (\%) & $1.25 \pm 0.19$ & $0.98 \pm 0.06$ & $7.7 \pm 1.2+\dagger$ & $3.7 \pm 0.6 t^{* *}$ & $0.61 \pm 0.18$ & $0.85 \pm 0.30$ & $8.9 \pm 2.3+\dagger$ & $9.6 \pm 2.3 \dagger$ \\
\hline
\end{tabular}

Data are presented as mean \pm SEM; Organ weights were normalized for baseline body weights (BW)

${ }^{*} p<0.05 ;{ }^{*} p<0.01 ;{ }^{* *}<0.001$ versus LNA control, $\uparrow p<0.05 ; \dagger+p<0.01 ; \dagger \uparrow \uparrow p<0.001$ versus sham 
Supplementary Table 7. Flow Cytometric Analysis of the Cardiac Infiltrate at Day 7 Post-infection

\begin{tabular}{|c|c|c|c|c|}
\hline & $\begin{array}{l}\text { LNA control } \\
\text { sham }\end{array}$ & $\begin{array}{l}\text { LNA-155 } \\
\text { sham }\end{array}$ & LNA control VM & $\begin{array}{l}\text { LNA-155 } \\
\text { VM }\end{array}$ \\
\hline Number & 4 & 4 & 8 & 8 \\
\hline T lymphocytes $\%$ of CD45+ & $12.4 \pm 1.3$ & $18.9 \pm 0.8 * *$ & $14.3 \pm 0.7$ & $16.3 \pm 1.7$ \\
\hline CD4+ lymphocytes & $59.8 \pm 0.9$ & $69.0 \pm 1.6 * *$ & $75.3 \pm 1.4$ ††† & $69.7 \pm 1.8 *$ \\
\hline Regulatory T lymphocytes & $8.8 \pm 0.8$ & $6.4 \pm 0.1 *$ & $15.1 \pm 1.3+\dagger$ & $16.1 \pm 1.0$ \\
\hline Naive CD4+ lymphocytes & $16.4 \pm 4.7$ & $25.8 \pm 5.9$ & $4.2 \pm 0.9+\dagger$ & $21.7 \pm 7.7 *$ \\
\hline Effector CD4+ lymphocytes & $79.9 \pm 5.0$ & $68.6 \pm 5.8$ & $93.5 \pm 1.1 \mathrm{t \dagger}$ & $73.5 \pm 8.4$ * \\
\hline CD8+ lymphocytes & $13.5 \pm 1.1$ & $18.6 \pm 1.6 *$ & $14.6 \pm 0.8$ & $22.5 \pm 1.4 * * *$ \\
\hline Naive CD8+ lymphocytes & $21.1 \pm 4.1$ & $35.6 \pm 7.6$ & $10.0 \pm 2.5+$ & $26.6 \pm 8.8 *$ \\
\hline Effector CD8+ lymphocytes & $76.5 \pm 4.7$ & $61.3 \pm 7.5$ & $87.4 \pm 2.9$ & $70.3 \pm 9.5$ \\
\hline B lymphocytes & $13.4 \pm 0.6$ & $12.1 \pm 1.0$ & $1.8 \pm 0.2$ †† & $3.2 \pm 1.4$ \\
\hline Granulocytes & $2.8 \pm 0.7$ & $3.1 \pm 0.5$ & $0.2 \pm 0.1$ t†† & $0.5 \pm 0.2$ \\
\hline Monocytes & $30.8 \pm 1.6$ & $18.8 \pm 0.5 * * *$ & $44.6 \pm 2.3+\dagger$ & $31.2 \pm 3.2 * *$ \\
\hline Pro-inflammatory monocytes & $21.2 \pm 3.6$ & $21.3 \pm 5.1$ & $67.3 \pm 1.4$ ††† & $65.7 \pm 7.3$ \\
\hline Macrophages & $13.8 \pm 1.0$ & $13.5 \pm 2.3$ & $2.5 \pm 0.2 \mathrm{tt \dagger}$ & $2.6 \pm 0.7$ \\
\hline
\end{tabular}

Data are presented as mean percentage of parent population \pm SEM

${ }^{*} p<0.05$; * $p<0.01$; ** $p<0.001$ versus LNA.control, $\dagger p<0.05$; †† $p<0.01$; $\uparrow+\uparrow p<0.001$ versus sham

Supplementary Table 8. Serial echocardiographic analysis of LNA control and LNA-155 mice

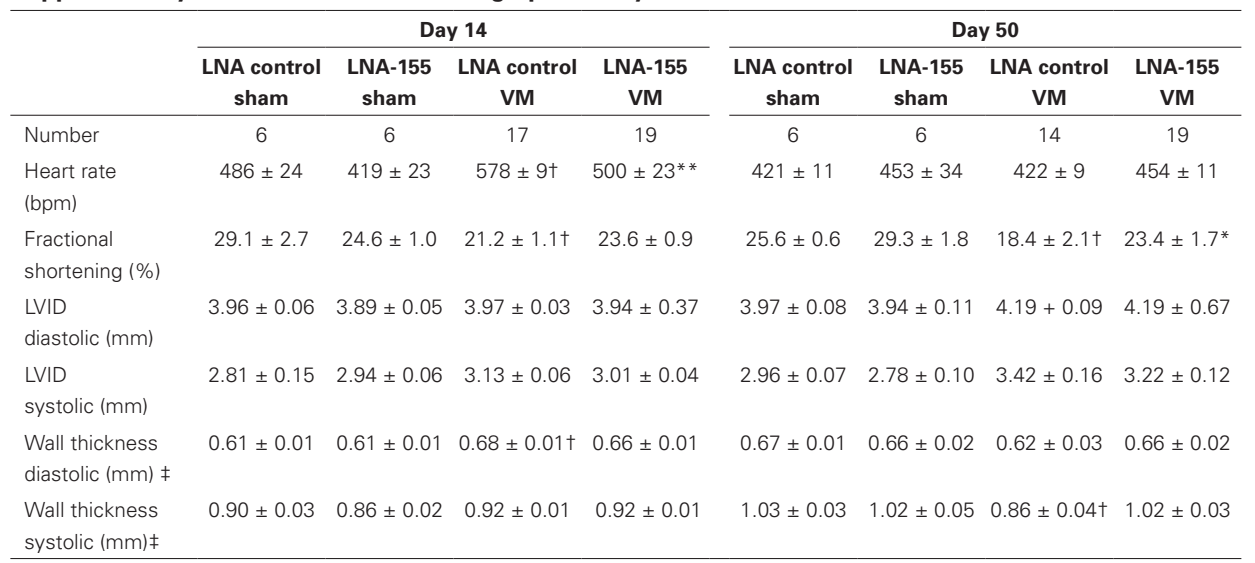

Data are presented as mean \pm SEM; LVID left ventricular internal dimension

${ }^{*} \mathrm{p}<0.05 ;{ }^{*} \mathrm{p}<0.01 ;{ }^{* * *}<0.001$ versus LNA control, $\mathrm{t} \mathrm{p}<0.05 ; \dagger+\mathrm{p}<0.01 ; \dagger+\uparrow \mathrm{p}<0.001$ versus sham, faveraged anterior and posterior wall thickness 


\section{References}

1. Chow LH, Gauntt CJ, McManus BM. Differential effects of myocarditic variants of coxsackievirus b3 in inbred mice. A pathologic characterization of heart tissue damage. Laboratory investigation; a journal of technical methods and pathology. 1991;64:55-64

2. Cooper LT, Jr. Myocarditis. N Engl J Med. 2009;360:1526-1538

3. Jorgensen S, Baker A, Moller S, Nielsen BS. Robust one-day in situ hybridization protocol for detection of micrornas in paraffin samples using Ina probes. Methods. 2010;52:375-381

4. Nuovo GJ. In situ detection of micrornas in paraffin embedded, formalin fixed tissues and the co-localization of their putative targets. Methods. 2010;52:307-315

5. Dunning MJ, Smith ML, Ritchie ME, Tavare S. Beadarray: R classes and methods for illumina bead-based data. Bioinformatics. 2007;23:2183-2184

6. Smyth GK. Limma: Linear models for microarray data. Bioinformatics and Computational Biology Solutions using $\mathrm{R}$ and Bioconductor. 2005

7. Irizarry RA, Bolstad BM, Collin F, Cope LM, Hobbs B, Speed TP. Summaries of affymetrix genechip probe level data. Nucleic acids research. 2003;31:e15

8. Dai M, Wang P, Boyd AD, Kostov G, Athey B, Jones EG, Bunney WE, Myers RM, Speed TP, Akil H, Watson SJ, Meng F. Evolving gene/transcript definitions significantly alter the interpretation of genechip data. Nucleic acids research. 2005;33:e175

9. De Windt LJ, Willemsen PH, Popping S, Van der Vusse GJ, Reneman RS, Van Bilsen M. Cloning and cellular distribution of a group ii phospholipase a2 expressed in the heart. J Mol Cell Cardiol. 1997;29:2095-2106 
Maarten F. Corsten ${ }^{1}$

Wouter Verhesen ${ }^{1}$

Anna Papageorgiou ${ }^{1}$

Paolo Carai ${ }^{1}$

Georg Summer ${ }^{1}$

Mark Hazebroek ${ }^{1}$

Fons Verheyen ${ }^{2}$

Rick van Leeuwen

Blanche Schroen ${ }^{1}$

Stephane Heymans 1,3,4 $^{1,4}$

1 Center for Heart Failure Research, Cardiovascular Research Institute Maastricht, The Netherlands

2 Electron Miscropscopy Unit, Maastricht University, The Netherlands

3 Interuniversity Cardiology Institute of the Netherlands, Utrecht, The Netherlands

4 Cardiovascular Sciences Department, University of Leuven, Belgium 


\section{The MicroRNA-221-222 Family Controls Viral Replication and Cardiac Inflammation in Acute Enteroviral Myocarditis}

In preparation

\section{ABSTRACT}

Rationale viral myocarditis is an important cause of heart failure and sudden cardiac death in young adults. Our previous microRNA profiling study has shown that many microRNAs are differentially expressed in the heart during viral myocarditis, including the family members microRNA-221 (miR-221) and miR-222.

Objective To study the functional effects of miR-221 and miR-222 expression during enteroviral myocarditis in mice.

Methods \& Results we measured cardiac microRNA levels during viral myocarditis with microRNA microarrays and qRT-PCR. Levels of miR-221 and miR-222 were significantly elevated during acute viral myocarditis in mice, and depressed in biopsies from viral myocarditis patients. In situ hybridization and in vitro experiments showed that both microRNAs are expressed by multiple cell types in the heart, including cardiomyocytes and endothelial cells, and are upregulated in neonatal rat cardiomyocytes (RCM) in response to enteroviral infection. In vivo inhibition of miR-221 and miRNA-222 by systemically delivered antagomirs aggravated cardiac injury and inflammation at day 7 post-infection, related to increased viral loads at day 4 post-infection. In line, overexpression of miRNA-221 and miR222 in RCM inhibited enteroviral replication. We identified and confirmed a set of conserved miR-221-222 targets, including the pro-inflammatory transcription factors ETS1, ETS2, the chemokine CXCL12, the type I interferon inhibitor IRF2, the T cell development factor TOX and the anti-apoptotic proteins BCL2L11 and BMF. De-repression of these targets following miR221-222 inhibition in VM may contribute to aggravated inflammation and cardiac injury.

Conclusions systemic inhibition of miR-221 and miR-222 increases cardiac viral loads and cardiac inflammation during acute viral myocarditis in mice. 


\section{Introduction}

Viral myocarditis (VM) is an inflammatory condition of the heart that causes heart failure and sudden death in young, previously healthy individuals, and evidence-based therapies for VM are currently lacking ${ }^{1,2}$. Myocarditis has been estimated to account for up to $12 \%$ of sudden cardiac deaths in patients below 40 years of age, and is found in almost $10 \%$ of biopsies from patients with unexplained heart failure ${ }^{3,4}$. Cardiac injury in VM is caused by infection of the heart by cardiotropic viruses, such as the enteroviral Coxsackievirus B3 (CVB3) or Parvovirus B19 (PVB19), which triggers an adverse immune response resulting in viral elimination as well as cardiac myocyte destruction, reparative fibrosis and heart failure. While immune activity is an important contributor to cardiac injury, broad inhibition of the immune response does not lead to clinical improvement in VM patients ${ }^{3,5}$. In contrast, a range of preclinical studies has recently indicated that not all components of the immune response are equally beneficial or detrimental during $\mathrm{VM}$, and that targeting specific signaling pathways may protect against cardiac inflammatory injury without compromising viral elimination ${ }^{2,4}$.

MicroRNAs (miRNAs) are important regulators of gene expression in a wide range of biological processes, including cardiac biology and the regulation of immune responses ${ }^{6-8}$. Recently, we studied miRNA expression profiles in the hearts of mice and humans during acute VM and identified a large number of up- and downregulated miRNAs at different time points of disease (this thesis, Chapter 4). Among the most strongly upregulated miRNAs were the miRNA family members microRNA-221 (miR-221) and miR-222. The co-transcribed miR-221-222 family was previously reported to promote tumor growth by targeting cell cycle inhibitors p27(kip1) and p57(kip2) ${ }^{9,10}$ and to affect vasculature by promoting smooth muscle cell proliferation and inhibiting angiogenesis ${ }^{11-13}$. However, the roles of miR-221 and miR-222 in the heart or during viral infection have not been studied so far. In this study, we aimed to investigate which cells express miR-221-222 in the heart and whether cardiac miR-221-222 expression during viral myocarditis is functionally important for the pathogenesis of cardiac injury in VM. We found that miR-221 and miR222 are expressed by cardiomyocytes, fibroblasts and endothelial cells in the heart, as well as by infiltrating inflammatory cells, and directly target a range of inflammatory mediators, including the transcription factors Protein C-ets-1 (ETS1), ETS2 and the chemokine (C-X-C motif) ligand 12 (CXCL12). Both miRNAs are upregulated in cardiomyocytes in response to infection with CVB3 and overexpression of miR-221-222 inhibited viral replication in cardiomyocytes in vitro. In vivo, systemic inhibition of miR221-222 by antagomirs aggravated cardiac injury and infiltration of T cells and macrophages in the heart during acute VM at day 7 post-infection, and caused increased early viral loads at day 4 post-infection. These data identify miR-221-222 expression as a protective factor in acute VM. 


\section{Methods}

\section{Animal studies}

All mouse experiments were performed according to the local guidelines for care and use of laboratory animals approved by the institutional animal care committee. For viral myocarditis (VM) experiments, 4-weeks-old male susceptible $\mathrm{C} 3 \mathrm{H}$ mice (Harlan, Boxmeer, The Netherlands) were inoculated intraperitoneally with $2 \times 10^{5}$ plaque forming units of Coxsackievirus B3 (CVB3) or saline. For microRNA knockdown in vivo, antagomirs were administered to male 4-week-old C3H mice intravenously at a dose of $40 \mathrm{mg} / \mathrm{kg}$ per injection (inhibition of miR-221-222 with 20/20 mg/kg versus $40 \mathrm{mg} / \mathrm{kg}$ control antagomiRs). Antagomirs were ordered from Fidelity Systems (Gaithersburg, MD) as fully 2'-O-methylated RNA oligonucleotides with perfect complementarity to the full mature sequences of miR221 or miR-222, a phosphorothioate backbone at both flanks, and a 3' cholesterol modification. Sequences: anti-mmu-miR-221: 5'-G*A*AACCCAGCAGACAAUGUA*G* ${ }^{*} *$ U*Chol* T-3'; anti-mmu-miR-222: 5'-G*A*GACCCAGUAGCCAGAUGUA* ${ }^{*}{ }^{*} \mathrm{C}^{*} \mathrm{U}^{*} \mathrm{Chol}^{*} \mathrm{~T}-3^{\prime}$ and anti-control $5^{\prime}-C^{*} A{ }^{*} G C U G A A G U A A A \cup A C C G A C *{ }^{*} A^{*} G{ }^{*} C h o l{ }^{*} T-3^{\prime}$ (* indicates phosphorothioate bond).

For analysis at day 7 post-infection, antagomiRs were given at days 1, 4 and 6 post-CVB3 infection and group sizes were at least $n=5$ for sham groups, and at least $n=10$ for $V M$ groups ( $n=20$ for antagomiR-221-222 VM and, $n=10$ for antagomiR-control VM and $n=20$ for PBS VM). For analysis at day 4 post-infection, antagomiRs were given at days 0 , and 2 post-CVB3 infection and group sizes were $n=3$ for sham groups and $n=7$ for VM groups. Organs were snap-frozen or fixed for 4-6 hours in 1\% paraformaldehyde for histology. Circulating leukocyte numbers were counted in fresh heparinized mouse blood samples using the Abbott Cell-Dyn 3700 (Wavre, Belgium).

\section{Patient material}

All human material was obtained during routine sampling used for clinical purposes, stored in a coded way and available for research purposes in accordance with the Declaration of Helsinki and the ethical committee at Maastricht University Medical Center. For microRNA profiling, right ventricular septal biopsies were used, obtained during routine clinical sampling from patients with acute myocarditis, in view of its recurrence or lack of improvement within 48 hours $(n=4)$. Patients with a definite clinical history of myocarditis were included' (Supplementary Table 1). Patients presented with an episode of upper airway or gastro-intestinal infection, followed after 1-3 weeks by chest pain. All myocarditis patients had elevated troponin T plasma levels and immunohistochemically proven cardiac inflammation ( $\mathrm{n}>14 \mathrm{CD} 45$ positive cells $/ \mathrm{mm}^{2}$ ). In all patients, virus presence was demonstrated in the cardiac biopsies (Supplementary Table 1). Coronary artery disease was excluded either by CAG or CT. Controls $(n=6)$ consisted of age-matched patients with unexplained ventricular tachy-arrhythmias but with a normal ejection fraction and the absence of systemic or cardiac inflammation or virus presence at the time of biopsy (Supplementary Table 1). Cardiac biopsies were immediately snap-frozen for RNA analysis or formalin-fixed for histological examination. 


\section{RNA isolation, microRNA arrays, northern blotting and quantitative RT-PCR}

Total RNA was isolated from left ventricular mouse tissue samples and right ventricular septal biopsies using the miRVANA isolation kit (Ambion, Warrington, UK), according to the manufacturer's instructions and without enrichment for small RNAs. Potential genomic DNA contamination was removed using the DNA-free kit (Ambion). For microRNA array analysis, total RNA from human biopsies was hybridized to Illumina Human miRNAv2 Expression Panel arrays by ServiceXS (Leiden, The Netherlands) and total RNA from mouse left ventricles was hybridized to $\mu$ Paraflo $₫$ arrays (LC Sciences, Houston, TX). For Northern blot analysis, 1-5 $\mu \mathrm{g}$ total RNA per sample were heated at 85 degrees Celsius for 5 minutes, loaded on a denaturing 20\% acrylamide gel and ran for 2 hrs at $100 \mathrm{~V}$. RNA was transferred to a nylon membrane (Roche, Mannheim, Germany) using a semi-dry blotting device (Biorad, Veenendal, The Netherlands) for 15 minutes at 20V. Blots were hybridized at $37^{\circ} \mathrm{C}$ with dual DIG-labeled LNA probes (Exiqon, Vedbaek, Denmark) and visualized using the DIG Wash and Block buffer set (Roche) and CDP-star reagent (Roche). For quantitative real-time PCR analysis, cDNA was generated using the miScript kit (Qiagen, Venlo, The Netherlands) and quantified using IO SYBR-green supermix (Bio-Rad) in a MylQ iCycler (Bio-Rad). LNA-based primer assays (Exiqon) were used for detection of mature microRNAs. A complete list of primer sequences is provided in Supplementary Table 2.

\section{In situ hybridization}

In situ hybridization was performed as described previously ${ }^{14,15} .4 \mu \mathrm{m}$ sections were prepared from $48 \mathrm{hr} 10 \%$ neutral buffered formalin fixed human or mouse cardiac biopsies. Sections were de-waxed, dehydrated, air-dried briefly and incubated in $1.3 \mathrm{mg} / \mathrm{ml}$ Pepsin $/ 0.1 \mathrm{mM} \mathrm{HCl}$ solution for $5 \mathrm{~min}$ at $37^{\circ} \mathrm{C}$. After a brief wash in DEPC MQ, sections were incubated overnight at $42.5^{\circ} \mathrm{C}$ in $1 \times$ hybridisation buffer (ENZO Life sciences, Farmingdal, NY, \#33808) with either 100 nM mmu-miR-221 or mmu-miR-222 DIG labeled probe (EXIOON, Vedbaek, Denmark, \#39471-01) or a scrambled control probe (\#99004-01). The following day the sections were washed once in 5xSSC and twice in 0.2xSSC at hybridisation temperature. Subsequently, sections were blocked in DIG blocking buffer from the DIG Wash and Block Buffer Set (Roche, Woerden, The Netherlands, \#11585762001) for 20 min followed by a 45 min incubation in 1:500 anti-DIG-AP, Fab fragments (Roche, \#11093274910). Sections were washed $3 \times 5$ min with TBS and incubated 3 min in DIG detection buffer at room temperature. AP signal was detected by using NBT/BCIP tablets (Roche, \#11697471001) for 4 hours at $30^{\circ} \mathrm{C}$ in darkness. Finally, sections were counterstained with fast red (Sigma, Zwijndrecht, The Netherlands) briefly washed in $\mathrm{MQ}$, quickly dehydrated through an ethanol gradient and mounted with entellan (EMS, Hatfield, PA, \#14800).

\section{Histology}

Paraffin-embedded $4 \mu \mathrm{m}$ LV sections were stained with hematoxylin-eosin for microscopic analysis. Left ventricular cryosections of $7 \mu \mathrm{m}$ were acetone-fixed and immunohistochemically stained by incubation with a monoclonal rat-anti-mouse antibody against CD11b (R\&D systems, Minneapolis, MN, \#MAB11124), or a polyclonal rat anti-mouse antibody against 
CD3 (gift from Dr. Menno de Winther). Photomicrographs were obtained (Leitz DMRXE; Leica, Wetzlar, Germany) and immunoreactivity was quantified as the number of positive cells per $\mathrm{mm}^{2}$, or when too many cells overlapped as stained area percentage, using OWin morphometry software (Leica).

\section{In vitro experiments}

Neonatal rat ventricular cardiac myocytes (RCMs) and fibroblasts (RCFs) were isolated by enzymatic disassociation of 1-to-2-day-old pups, plated on gelatinized 6-well plates and cultured as previously described ${ }^{16}$. Per well, $10^{6} \mathrm{RCMs}$ or $2^{*} 10^{5} \mathrm{RCFs}$ were grown on high serum media for 48 hours. They were then put on low serum media overnight before stimulation with $10 \mathrm{ng} / \mathrm{mL}$ TNF- $\alpha$ (PeproTech, Rocky Hill, NJ, \#315-01A), 10ng/ $\mathrm{mL}$ II-1 $\beta$ (PeproTech, \#211-11B) for 6/24 hours, or infection with coxsackievirus B3 at an $\mathrm{MOI}$ of 1 . Experiments with murine cardiac endothelial cells (MCEC) were performed under similar conditions in 12-well plates, seeding $2.5^{*} 10^{5}$ cells per well. Bone marrowderived macrophages (BMMs) were harvested from the femurs and tibias of adult $\mathrm{C} 3 \mathrm{H}$ mice and placed in R10 (RPMI 1640 medium, containing 10\% heat-inactivated fetal calf serum, $100 \mathrm{U} / \mathrm{ml}$ penicillin, $100 \mu \mathrm{g} / \mathrm{ml}$ streptomycin, $2 \mathrm{mM}$ L-glutamine, and $10 \mathrm{mM}$ Hepes) supplemented with $15 \%$ L-929 cell-conditioned medium. After 7 days, cells were scraped and seeded in 6-wells at $10^{6}$ cells per well. After incubation overnight in low serum media, BMMs were stimulated with $10 \mathrm{ng} / \mathrm{mL}$ lipopolysaccharide (from E. coli 055.B5; Sigma) for 24 hours. Primary T cells were isolated from adult mouse spleens by mechanical dissociation, followed by magnetic bead separation of CD4/8 positive cells by negative selection using the MACS Pan T cell isolation kit (Miltenyi Biotec, Auburn, CA, USA, \#130-095-130). Cells were seeded in 6-wells at $10^{6}$ cells per well and stimulated with $5 \mu \mathrm{g} / \mathrm{mL}$ concanavalin A (Sigma, \#C5275) for 24 hours. After stimulation RNA was isolated with miRVana (Ambion) and quantitative PCR was performed as described above.

To assess the effect of miR-221-222 overexpression or inhibition in vitro, RCMs, RCFs, or MCECs were grown on high serum media for 24 hours before transfection using lipofectamine 2000 (Invitrogen, Breda, The Netherlands, \#11668-019) according to manufacturer's instructions. Cells were transfected with pre-miRs (MicroRNA mimics, Thermo Scientific Dharmacon, Lafayette, CO) for rno-miR-221 (C-032421-05), rno-miR-222 (C-320420-03) or negative control (CN-002000-01) at 20/20nM for miR-221-222 and 40nM for control; or with miRNA inhibitors (miRVANA inhibitors against mmu-miR-221-3p (Ambion, \#4464084-MH10337), mmu-miR-222-3p (Ambion, \#4464084-MH11376), or negative control (Ambion \#4464076) at 40/40nM for miR-221-222 and 80nM for control. After 6 hours, medium was replaced and cells were put on low serum media overnight before infection with $\mathrm{CVB} 3$ at $\mathrm{MOI}=1$ or stimulation with TNF- $\alpha$ for $6 / 24$ hours. To assess $\mathrm{nRCM}$ viability after CVB3 infection after 120 hours, cells were washed twice with PBS followed by lysis using the CellTiter-Glo Luminescent Cell Viability Assay (Promega, Madison, WI, G7570) and read on a Spectramax L luminescent plate reader (Molecular Devices, Sunny Ville, CA). 


\section{Luciferase reporter assays}

To confirm direct targeting of candidate targets by miR-221 and miR-222, we performed luciferase reporter assays. Mouse $3^{\prime}$ UTR fragments $(\sim 1 \mathrm{~kb})$ were PCR amplified (Takara LA Taq, Clontech, Mountain View, CA) and cloned into the firefly luciferase $3^{\prime} U T R$ of pMIR-REPORT (Applied Biosystems), using renilla luciferase as an internal control. Cloning primer sequences are provided in Supplementary Table 3. Mutations in th putative miR221-222 binding sites were introduced using site-directed mutagenesis (Quikchange II $\mathrm{XL}$, Stratagene, La Jolla, CA), replacing the ATGTAGCA seed-binding sequence with ATGTCTAA (mutated bases underlined. Mutation primer sequences are given in Supplementary Table 4. Reporter plasmids were contransfected with premiRs (MicroRNA mimics, Thermo Scientific Dharmacon), into HEK293 cells using Lipofectamine 2000 (Invitrogen) in 96-well plates (per well: $0.5 \mu \mathrm{L}$ Lipofectamine 2000, 50ng plasmid, 1/1nM for pre-miR-221/pre-miR-222 versus $2 \mathrm{nM}$ for pre-miR-29b or pre-negative control (NC)). Luciferase was read after 24 hours using the Dual-Glo kit (Promega) on a Spectramax L luminescent plate reader (Molecular Devices). Measurements were performed in technical triplicates and were performed at least twice.

\section{Electron microscopy}

Small tissue samples of the left ventricle were immersed in $3 \%$ glutaraldehyde in $\mathrm{KH}_{2} \mathrm{PO}_{4}$ buffer (pH7.4), postfixed in $1 \% \mathrm{OsO}_{4}$ in veronal acetate buffer $(\mathrm{pH} 7.4)$ supplemented with $1.5 \%$ ferricyanide and routinely processed for embedding in epoxy resin.

\section{Statistical analyses}

Statistical analyses for miRNA arrays were performed in R. Analyses for experimental work were performed using GraphPad Prism 5.0d for Macintosh (GraphPad Software Inc., La Jolla, CA, USA). Comparisons between 2 groups were performed with two-tailed Student's t testing for Gaussian data or Mann-Whitney tests for non-Gaussian data. For comparisons of more than 2 groups, one-way ANOVA was used, followed by post hoc testing using Bonferroni correction for more groups. Probability values below 0.05 were considered statistically significant. Data are presented as mean \pm SEM unless otherwise indicated.

\section{Results}

\section{Cardiac expression of the miR-221-222 family is increased during acute VM in mice} We studied the cardiac expression of miRNAs during VM in two mouse models. $\mathrm{C} 3 \mathrm{H}$ mice are susceptible to the development of VM after inoculation with CVB3, while C57BI/6 mice are resistant to cardiac inflammation despite similar viral titers. MiRNA levels were analyzed at baseline (day 0), during high viral presence before the immune infiltration (day 4) (as described in Chapter 4), and during the acute inflammatory phase (day 7). Microarray results for cardiac miR-221 and miR-222 expression at these time points are shown in Figure 1A. 
- $\mathrm{C} 57 \mathrm{~B} / / 6 \quad \square \mathrm{C} 3 \mathrm{H}$

a

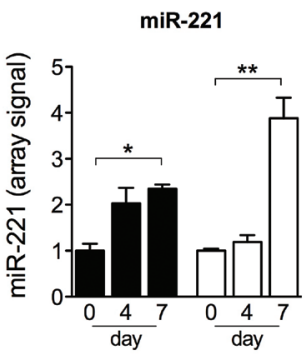

- $\mathrm{C} 57 \mathrm{BI} / 6 \quad \square \mathrm{C} 3 \mathrm{H}$

C

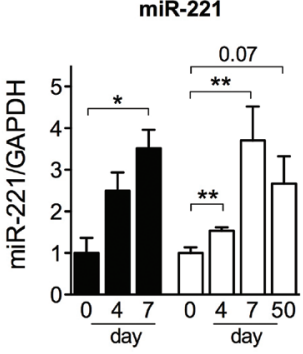

miR-222

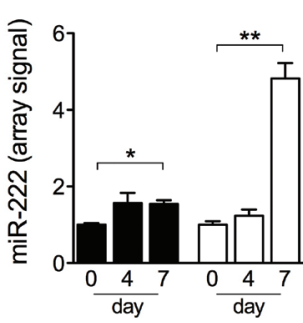

d

miR-222

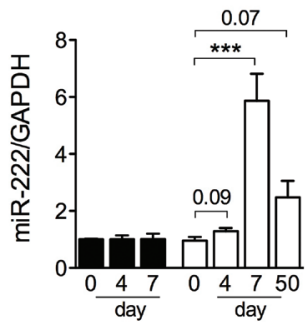

b
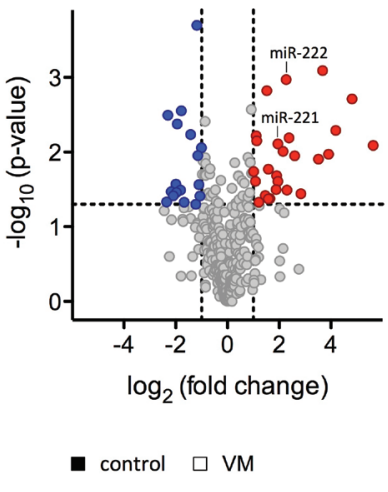

miR-221

miR-222
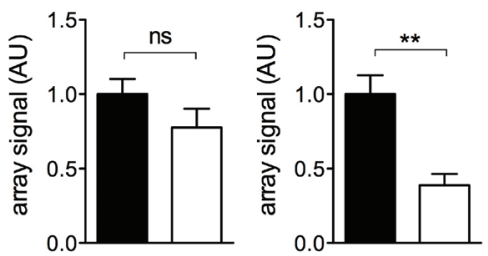

Figure 1. MicroRNA-221 (miR-221) and miR-222 are upregulated in mouse viral myocarditis (VM), and downregulated in human VM. (A) Microarray signal fold changes compared to baseline of cardiac miR-221 and miR-222 expression at day 4 (before cardiac immune infiltration) and day 7 (acute inflammation) postinfection with Coxsackievirus B3 (CVB3). Data are shown for both VM-resistant C57BI/6 and VM-susceptible $\mathrm{C} 3 \mathrm{H}$ mice. (B) Volcano plots showing fold changes (log2 values) and p values (log10) compared to baseline for individual miRNAs in $\mathrm{C} 3 \mathrm{H}$ mice at day 7 post-infection. (C) Quantitative real-time PCR measurement of miR-221 and miR-222 expression confirmed elevated levels of both miRNAs in C3H mice at day 7, and additionally showed elevation of miR-221 at day 4 in $\mathrm{C} 3 \mathrm{H}$ mice and at day 7 in C57BI/6 mice. (D) Microarray signal fold changes compared to control of cardiac miR-221 and miR-222 expression in cardiac biopsies from VM patients, showing trend-wise and significant downregulation of miR-221 and miR-222, respectively. ${ }^{*} \mathrm{P}$ $<0.05,{ }^{*} \mathrm{P}<0.01$, ${ }^{* *} \mathrm{P}<0.001$.

Levels of both miRNAs are significantly elevated at day 7 after CVB3 infection as compared to baseline in $\mathrm{C} 57 \mathrm{BI} / 6$ as well as in $\mathrm{C} 3 \mathrm{H}$ mice, and were among the most strongly upregulated miRNAs in the $\mathrm{C} 3 \mathrm{H}$ miRNA VM profile (Figure 1B). Quantitative real-time PCR (qRT-PCR) confirmed the elevation of both miRNAs in susceptible $\mathrm{C} 3 \mathrm{H}$ mice at day 7 (miR221: 3.7-fold, $\mathrm{p}<0.01$; miR-222: 5.9-fold, $\mathrm{p}<0.001$ ) and of miR-221 in C57BI/6 mice at day 7 (3.5-fold, $\mathrm{p}<0.05$ ) (Figure 1C). In addition, levels of miR-221 were already elevated in $\mathrm{C} 3 \mathrm{H}$ mice at day 4 (1.3-fold, $\mathrm{p}<0.01)$ whereas miR-222 levels were elevated by trend (1.3-fold, $\mathrm{p}=0.09$ ). Levels of both miRNAs remained elevated by trend in VM hearts after 50 days (miR-221: 2.7-fold, $p=0.07$; miR-222: 2.5-fold, $p=0.07$ ), after viral elimination and fibrotic remodeling (as described in Chapter 4). Together, these data indicate that miR-221 and miR-222 are strongly elevated during acute VM. 


\section{Cardiac expression of the miR-221-222 family is depressed in biopsies from patients with VM}

In contrast to their elevation in mice, miR-221-222 were not elevated in cardiac biopsies of VM patients. MiR-221 levels were depressed by trend as compared to controls (0.8-fold, $\mathrm{p}=0.2$ ) (Figure 1D), while miR-222 were significantly lower in VM $(0.4$-fold, $p<0.01)$. Though the causative agent in the VM patients from Figure 1D was PVB19, both miRNAs are also depressed in biopsies from VM patients with other viral pathogens, including CVB3 and adenoviruses (personal communication, Wolfgang Poller), indicating that miR-221-222 downregulation is not virus-specific. In fact, miR-221 and miR-222 downregulation was previously described in hearts of patients with ischemic cardiomyopathy and dilated cardiomyopathy ${ }^{17-19}$. While the difference between miR-221-222 regulation in humans and mice may indicate different sampling time-points during disease (acute in mice versus sub-acute/chronic in patients), it may also reflect non-conserved regulatory elements in the promoter. MiR-221 and miR-222 are located $~ 850$ bases apart and are co-transcribed

a

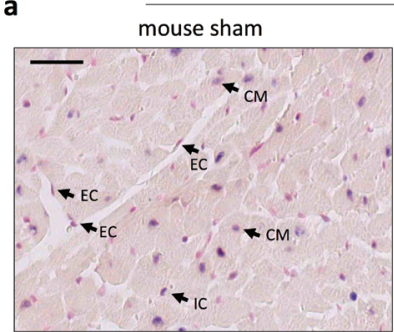

$\square$ control

$\square \mathrm{MOI}=1$

- $\mathrm{MOI}=5$

b

$\mathrm{RCM}+\mathrm{CVB3}$
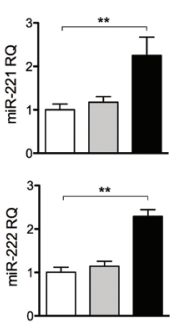

$\square$ control

$\square 6 \mathrm{~h}$

- $24 \mathrm{~h}$
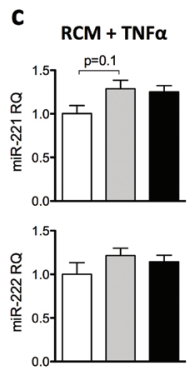

miR-221

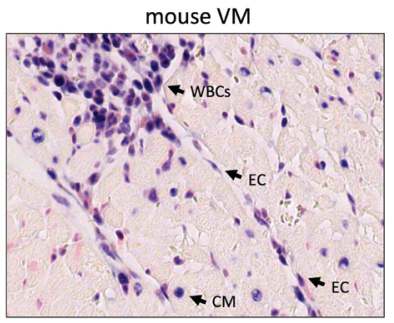

RCF + TNF $\alpha$

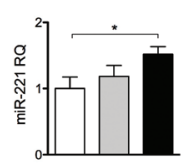

MCEC + TNFa

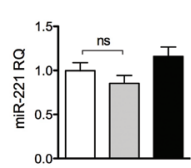

BMM + LPS

T cells + ConA
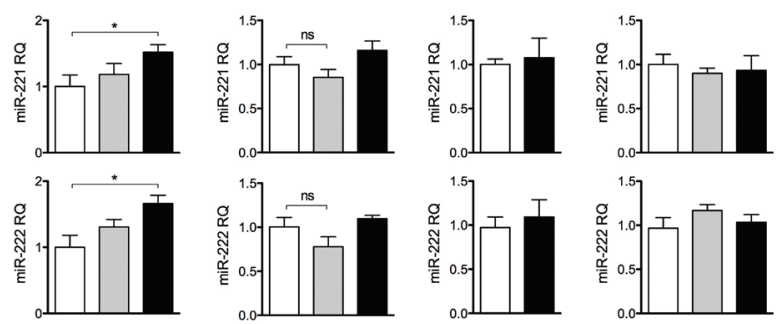

Figure 2. MiR-221 and miR-222 are expressed by multiple cell types in the heart. (A) In situ hybridization of miR-221 (blue) on left ventricular sections of a healthy mouse, VM mouse and a VM patient, showing blue cellular staining in multiple cardiac cell types, including cardiomyocytes (CM), endothelial cells (EC) and infiltrating white blood cells (WBC). Panel (B) shows miR-221 and miR-222 expression in responses to CVB3 infection or inflammatory stimulation in a cell panel including rat neonatal myocytes (RCM), fibroblasts (RCF), adult mouse cardiac endothelial cells (MCEC), primary bone marrow-derived macrophages (BMM) and splenic T cells. Inflammatory stimuli were TNF- $\alpha$ for RCMs, RCFs and MCECs, lipopolysaccharide (LPS) for BMMs and Concanavalin A (ConA) for T cells ( $n=3$ per group). Expression of miR-221 and miR-222 is significantly induced by CVB3 infection in RCM, and by TNF- $\alpha$ stimulation in RCFs. Expression levels are presented as relative to control. Scale bars: $50 \mu \mathrm{m}$. ${ }^{*} \mathrm{P}<0.05,{ }^{*} \mathrm{P}<0.01$. 
from a large intergenic region on the X-chromosome. Mature miR-221-222 sequences are perfectly evolutionary conserved from zebrafish to rodents and humans (Supplementary Figure 1A). Regulatory elements for miR-221-222 transcription - identified by H3K27AC regulatory element footprints and DNAsel hypersensitivity clusters - are located over a region spanning from 80 kilobases $(\mathrm{kb})$ upstream to $50 \mathrm{~kb}$ downstream of the miR-222 transcription start site (UCSC Genome browser analysis shown in Supplementary Figure 1B). This region includes no $\mathrm{CpG}$ islands but harbours $>200$ binding sites for transcription factors including several for nuclear factor kappa B (NF-kB), signal transducer and activator of transcription 3 (STAT3), and other inflammatory transcription factors (ENCODE Chip-seq data, not shown). Sequence conservation in this region is imperfect and may explain differential activation of miR-221 and miR-222 expression between different species.

\section{MiR-221-222 are expressed by multiple cardiac cell types and are upregulated in cardiomyocytes after CVB3 infection}

We next asked what cardiac cell types express miR-221 and miR-222 under basal and inflamed conditions, both in vivo and in vitro. In situ hybridization of miR-221 in the normal mouse heart revealed its expression by multiple cell types in the heart, including cardiomyocytes, endothelial cells, and interstitial cells (Figure 2A, miR-221 staining in dark blue) while no signal was detected using a scrambled probe (not shown). During VM (7 days), staining was again apparent in cardiomyocytes and endothelial cells, and in addition in recruited inflammatory cells (Figure 2A). Similarly, in cardiac biopsies from VM patients, we observed miR-221 expression both in infiltrating inflammatory cells and in cardiomyocytes. Unfortunately, we could not detect miR-222 with the commercial probe indicated in the methods, but, given their similar expression behaviour and common promoter, a deviant expression profile for miR-222 is not expected.

To assess whether miR-221 and miR-222 are regulated by infectious or inflammatory conditions in vitro, we infected isolated RCM with increasing concentrations of CVB3. Alternatively, we stimulated rat neonatal cardiomyocytes (RCM), fibroblasts (RCF), or murine cardiac endothelial cells (MCEC), with Tumor Necrosis Factor-alpha (TNF- $\alpha$ ) for 6 or 24 hours, bone marrow-derived macrophages (BMM) with lipopolysaccharide (LPS) for 24 hours, or splenic T lymphocytes with ConCanavalin A (ConA) for 6 or 24 hours. Cellular responses to stimuli were verified by measurement of responsive inflammatory genes (Supplementary Figure 2). We found that both miR-221 and miR-222 were upregulated by CVB3 infection in a dose dependent manner (CVB3 multiplicity of infection (MOI)=5; miR221: 2.3-fold, $p<0.01$; miR-222: 2.3-fold, $\mathrm{p}<0.01$ ) (Figure 2B). In contrast, the expression of miR-1 and miR-208a was unchanged (Supplementary Figure 2A). MiR-221-222 expression responses to TNF- $\alpha$ stimulation in cardiac cell types were mild: both miRNAs were upregulated in RCFs after $24 \mathrm{~h}$ of TNF- $\alpha(p<0.05)$ and slightly elevated by trend in RCMs. In contrast, TNF- $\alpha$ caused no miR-221-222 expression changes in endothelial cells, macrophages and $T$ cells, in line with previous reports ${ }^{20,21}$. Similar results were obtained by stimulation of RCMs, RCFs and MCECs with interleukin-1 beta (IL-1 $\beta$ ), and after TNF- $\alpha$ stimulation of the fibroblast 3T3 cell line and the endothelial cell line SVEC (Supplementary Figure 3). In conclusion, miR-221 and miR-222 are expressed by cardiomyocytes, fibroblasts, 
endothelial cells, and inflammatory cells in the VM heart. MiR-221-222 expression increases during murine VM likely reflect a combination of their upregulation in cardiomyocytes and fibroblasts, and of the influx of miR-221-222-expressing macrophages and T cells.

\section{In vivo inhibition of miR-221-222 increases cardiac injury and immune cell infiltration during acute viral myocarditis}

To investigate whether expression of miR-221-222 affects the course of VM in mice, we systemically administered antagomirs against miR-221-222, a control sequence, or phosphate buffered saline (PBS), at 3 time points throughout the first 7 days of VM in male $\mathrm{C} 3 \mathrm{H}$ mice (Figure 3A). qRT-PCR analysis of miR-221 and miR-222 expression confirmed upregulation of both miRNAs during VM in antagomir groups and revealed strong and significant knockdown of both miRNAs in sham and VM conditions after antagomir-221-222 administration (Figure 3B). We observed no effects of the control antagomirs as compared to PBS treatment. Using northern blotting for mature miR-221 under denaturing conditions, we detected sharply reduced miR-221 levels after antagomir-221-222 treatment, indicative of knockdown by miRNA degradation (Figure 3C). While no mortality was observed in PBS or antagomir control-treated VM animals, 3 out of 20 antagomiR-221-222-treated VM animals died during the first week after infection (Supplementary Table 5). At day 7 ,

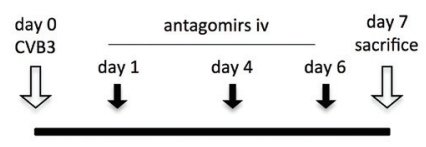

b

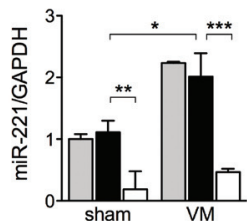

C

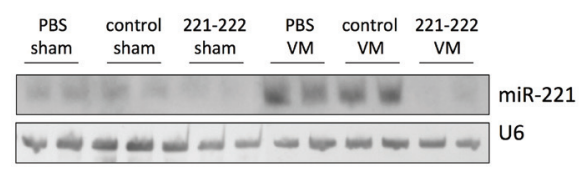

d

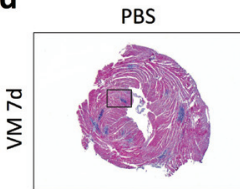

antagomir control

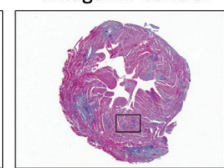

antagomir-221-222
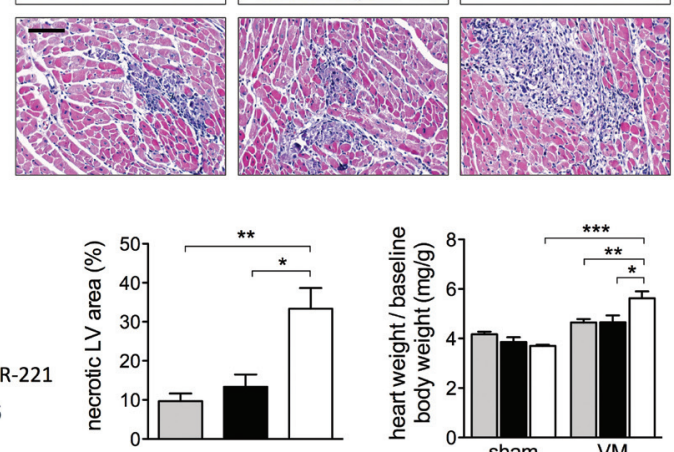

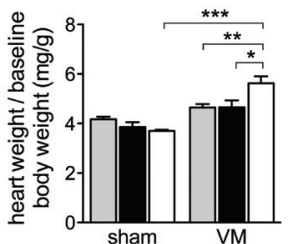

Figure 3. In vivo inhibition of miR-221 and miR-222 aggravates cardiac injury during acute viral myocarditis. (A) Study design: antagomirs targeting miR-221 and miR-222 were administered by three intravenous injections throughout the first 7 days of CVB3-induced VM. (B) qRT-PCR analysis of cardiac miR221-222 expression, demonstrating significant knockdown of both miRNAs by antagomir treatment in both sham and VM groups. In agreement, northern blot analysis showed markedly reduced band intensity after antagomir treatment (C). (D) Left ventricular H\&E stainings at day 7 of CVB3-induced VM showing aggravated cardiomyocyte necrosis after miR-221-222 inhibition (LV necrotic area 33.3\% in antagomiR-221-222 VM, compared to $9.7 \%$ in PBS VM, p<0.01; and $13.4 \%$ in antagomir control VM, $\mathrm{p}<0.05$ ). In addition, miR-221222 inhibition significantly promoted heart weight increase during VM as a marker of cardiac edema. $(n \geq 5$ per sham group and $n \geq 10$ per VM group). Scale bar: $100 \mu \mathrm{m}$. ${ }^{*} \mathrm{P}<0.05,{ }^{*} \mathrm{P}<0.01,{ }^{*}{ }^{*} \mathrm{P}<0.001$. 

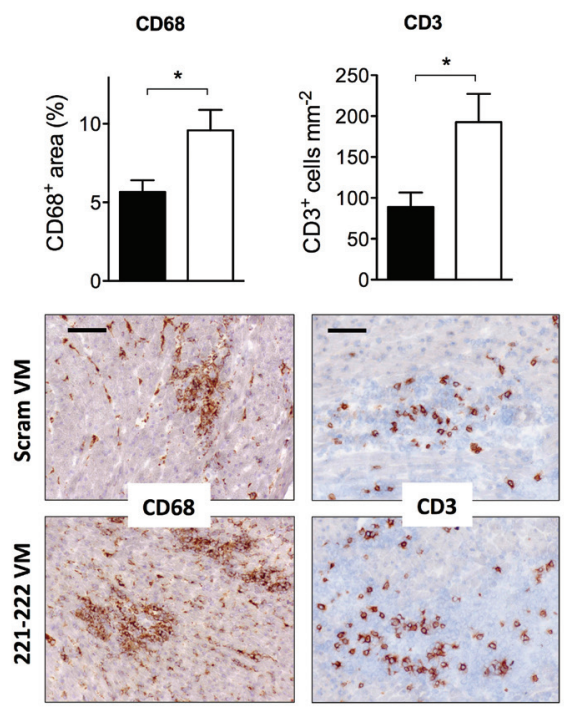

b
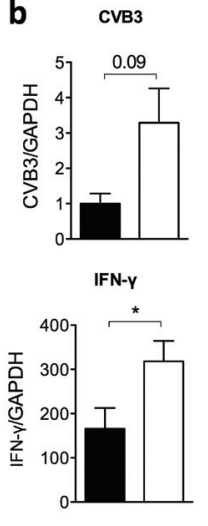

ICAM1

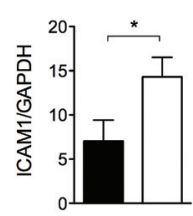

IL-6
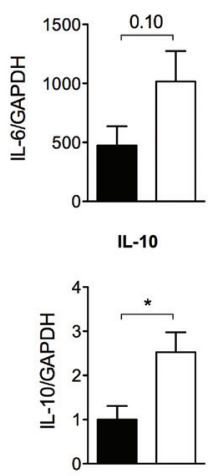

MCP1

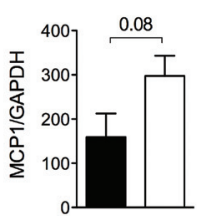

TNF- $\alpha$
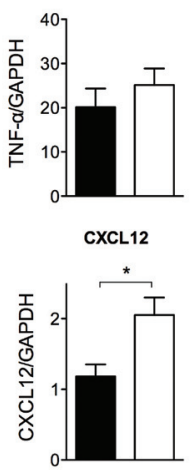

PSEL

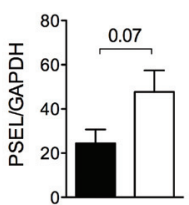

Figure 4. Inhibition of miR-221-222 promotes cardiac immune cell infiltration and inflammatory cytokine expression in VM. (A) MiR-221-222 inhibition promoted cardiac infiltration of CD68+ macrophages $(p<0.05)$ and CD3+ T lymphocytes $(p<0,05)$ during VM. (B) qRT-PCR analysis showing that miR-221-222inhibited VM hearts contained more than 3 -fold higher CVB3 genome copies $(p=0.09)$ than VM controls, and expressed significantly higher amounts of both pro-inflammatory (interferon gamma, IFN- $\gamma$ ) as well as anti-inflammatory (IL-10) T cell-cytokines. MiR-221-222 inhibition also caused significantly higher expression levels of the chemokine CXCL12 and Intercellular Adhesion Molecule-1 (ICAM1), and elevation by trend of IL-6, Macrophage Chemoattractant Protein-1 (MPC1), and P-selectin (PSEL), while not affecting TNF- $\alpha$. ( $n \geq 5$ per sham group and $n \geq 10$ per VM group). Scale bars: $100 \mu m$. ${ }^{*} P<0.05$.

cardiac necrosis as measured on haematoxylin-eosin staining was significantly aggravated in antagomir-221-222 treated animals as compared to controls (33.3\% of left ventricular area in antagomiR-221-222 VM, compared to 9.7\% in PBS VM, $\mathrm{p}<0.01$; and $13.4 \%$ in antagomir control VM, p<0.05) (Figure 3D). In addition, miR-221-222 inhibition significantly enhanced heart weight increases during VM, a marker of cardiac inflammatory edema (Figure 3D and Supplementary Table 6). Immunostainings revealed that miR-221-222 inhibition promoted the infiltration of both CD3+ T cells and CD68+ macrophages in the heart after VM, while not affecting basal macrophage numbers in sham hearts (Figure 4A and Supplementary Figure 4B). In addition, miR-221-222 inhibition increased the white blood cell count in the circulation of VM mice (Supplementary Figure 4A). Electron microscopy confirmed necrotic degeneration of cardiomyocytes and influx of mononuclear cells in antagomir-221-222 VM hearts, while no ultrastructural differences were observed between sham hearts of different treatments (Supplementary Figure 5).

qRT-PCR revealed that miR-221-222-inhibited VM hearts contained more than 3-fold higher CVB3 genome copies $(p=0.09)$ than VM controls, and expressed significantly higher amounts of both pro-inflammatory (interferon gamma, IFN- $\gamma$ ) as well as anti-inflammatory 
(IL-10) T cell-cytokines (Figure 4B). MiR-221-222 inhibition also caused significantly higher expression levels of the chemokine CXCL12 and of Intercellular Adhesion Molecule-1 (ICAM1), and elevation by trend of IL-6, Macrophage Chemoattractant Protein-1 (MCP1), and P-selectin (PSEL), while not affecting TNF- $\alpha$ and IL-4 levels (Figure 4B and Supplementary Figure 4 C). Together, these data show that inhibition of miR-221 and miR-222 during VM in mice aggravates cardiac injury and inflammatory infiltration by macrophages and T cells, associated with increased expression of adhesion molecules, chemotaxis and viral load.

\section{Inhibition of miR-221-222 increases cardiac CVB3 load at day 4 post-infection}

Since miR-221-222-inhibited mice displayed higher cardiac CVB3 levels at day 7 of VM, we next investigated whether antagomirs against miR-221-222 directly affected viral loads in vivo. To circumvent possible confounding effects of inflammation on viral replication ${ }^{2}$, we analyzed the hearts of animals treated with antagomir-221-222 versus antagomir control at 4 days post-infection, before the onset of inflammatory infiltration (Supplementary Table 6 Table 2). Inflammatory patches were absent in all infected hearts, and both miR-221 and miR-222 were efficiently suppressed by antagomir administration (Figure 5A and B). At day 4, miR-221-222 inhibition significantly increased CVB3 titers in the heart (2.7-fold, $p<0.01$ ), without affecting the number of apoptotic cells in the myocardium (Figure 5C and D).

a

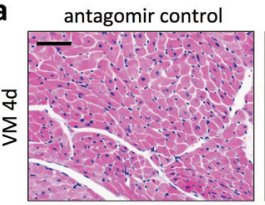

antagomir-221-222

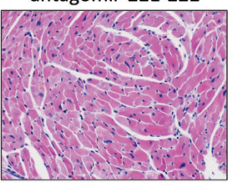

C

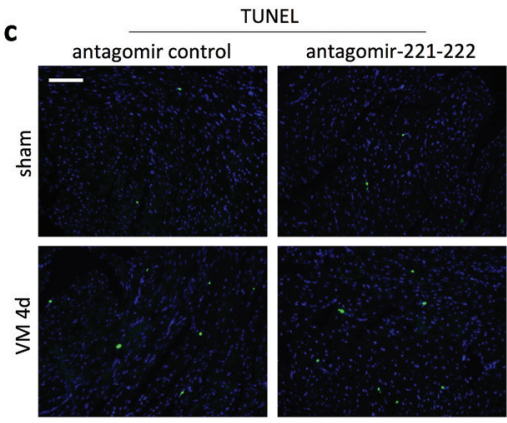

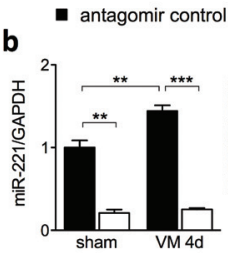

$\square$ antagomir-221-222

d

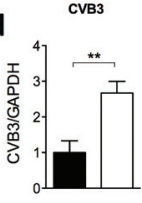

ICAM1
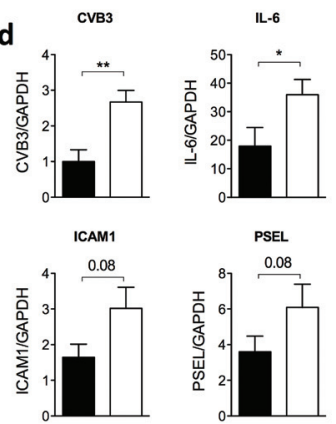

PSEL

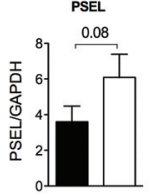

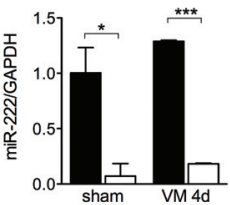

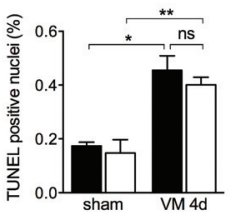

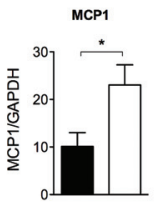

CXCL12

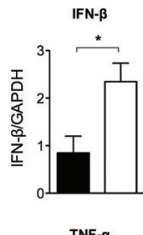

TNF- $\alpha$

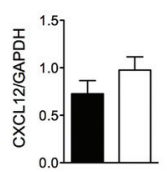

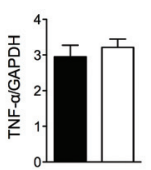

Figure 5. Inhibition of miR-221-222 increases cardiac CVB3 load at day 4 post-infection. (A) Left ventricular H\&E stainings at day 4 of CVB3-induced VM show absence of inflammatory patches at this time point. (B) qRT-PCR measurements of cardiac miR-221 and miR-222 levels, showing significant knockdown for both miRNAs. (C) Photographs and quantification of Terminal deoxynucleotidyl transferase dUTP nick end labeling (TUNEL, green signal with DAPI nuclear counterstain in blue). No differences in the percentage of cardiac apoptotic cells were observed between antagomir treatments. (D) qRT-PCR showing significantly higher CVB3 levels in antagomir-221-222-treated VM hearts compared to controls (2.7-fold, p<0.01). In addition miR-221-222 inhibition caused significantly higher expression levels of IL-6, MCP1, IFN- $\beta$, and with a trend to higher expression of adhesion molecules ICAM1 and P-selectin, as well as CXCL12 and II-1 $\beta$, but not TNF- $\alpha$. ( $n=5$ per sham group and $n=7$ per VM group). ${ }^{*} P<0.05$, ${ }^{*} P<0.01$, ${ }^{*}{ }^{*} P<0.001$. 

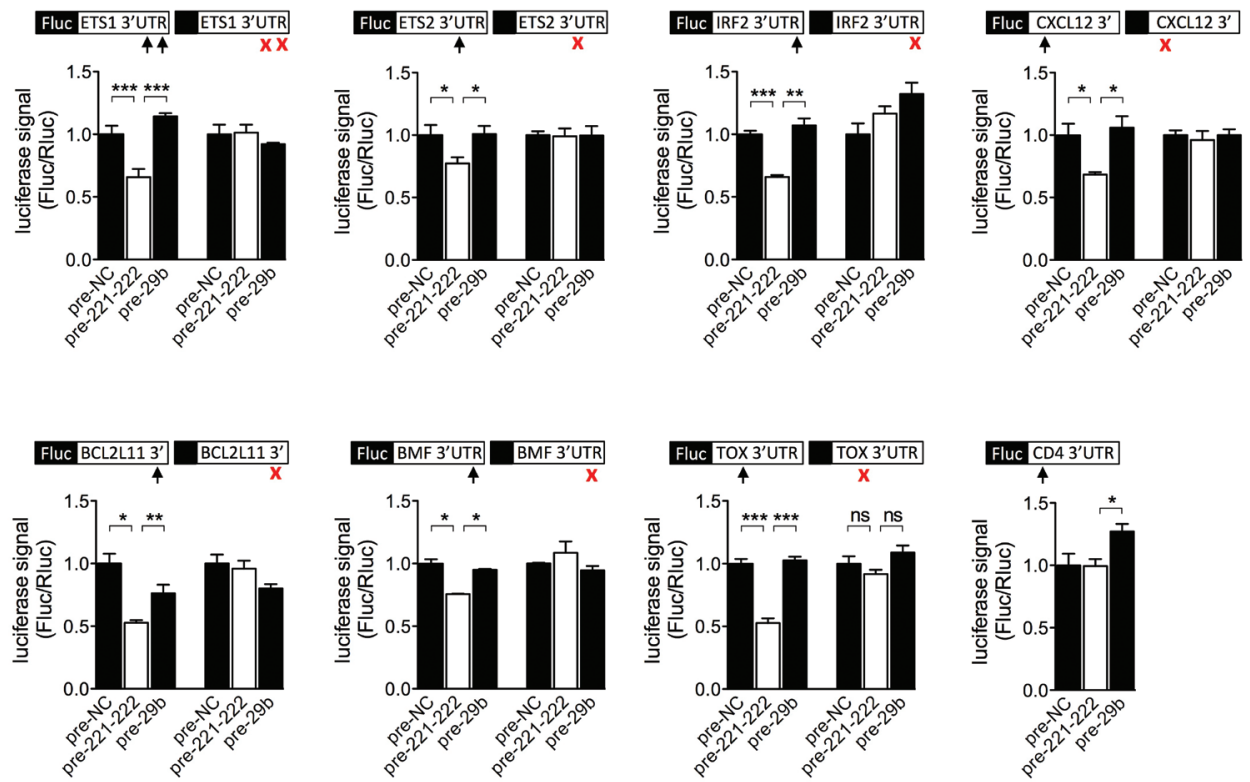

Figure 6. MiR-221 and miR-222 directly target multiple pro-inflammatory and anti-apoptotic proteins. Potential miR-221-222 targets were selected with evolutionary conserved predicted binding site for the miR-221-222 family in their 3'UTR, and with possible roles during viral myocarditis. Targets included the pro-inflammatory transcription factors ETS1 and ETS2, the inhibitor of type I interferons transcription IRF2, the chemokine CXCL12, the anti-apoptotic proteins BCL2L11 and BMF, and the T cell factors TOX and CD4. 3'UTR fragments containing the predicted binding sequence were cloned into pmiR-REPORT downstream of firefly luciferase (Fluc), using renilla luciferase (Rluc) as an endogenous control. Arrowheads indicate the location of predicted binding sites and red crosses indicate mutated sites. All predicted targets except for CD4 were regulated by miR-221-222, as indicated by significantly lower Fluc/Rluc ratios in presence of miR-221-222 (pre-221-222) overexpression but not by overexpression of miR-29b (pre-29b) or a negative control mimic (pre-NC). Mutation of 3 bases in the predicted binding sites abrogated target repression by miR-221-222 overexpression. ( $n=3$ per group; all experiment were performed at least twice). ${ }^{*} P<0.05,{ }^{*} P$ $<0.01,{ }^{* *} \mathrm{P}<0.001$.

Increased viral loads after miR-221-222 inhibition were associated with significantly higher expression levels of IL-6, MCP1, IFN- $\beta$, and with a trend to higher expression of adhesion molecules ICAM1 and P-selectin, as well as CXCL12 and II-1 $\beta$, but not TNF- $\alpha$ or VCAM, in line with the fact that cardiomyocytes efficiently produce IL-6 and MCP1 but only minimal amounts of TNF- $\alpha$ (Figure 5D and Supplementary Figure 6). These data indicate that miR221-222 directly impacts viral replication and cardiac CVB3 load before the inflammatory phase in the heart.

\section{The miR-221-222 family directly targets regulators of inflammation and apoptosis}

To identify direct miR-221-222 targets that may explain the aggravated cardiac inflammation and injury after miR-221-222 inhibition, we queried predicted miR-221-222 targets in mouse and human using the Targetscan algorithm and searched the overlapping (conserved) gene set for putative targets with a potential role during VM. We selected 8 potential targets: the pro-inflammatory transcription factors ETS1 and ETS2 promote the expression of 
cytokines, adhesion molecules and chemokines such as MCP ${ }^{122-24}$. In addition, ETS1 promotes replication of the human immunodeficiency virus-1 (HIV1) and human T cell leukemia virus 1 (HTLV1) in lymphoid cells ${ }^{25-27}$. Interferon Regulatory Factor-2 (IRF2) inhibits the transcription of type I interferons, which are protective in $\mathrm{VM}^{28}$. The chemokine CXCL12 is a strong lymphocyte attractant, while Thymocyte selection-associated high mobility group box protein (TOX) and CD4 are important in T cell biology. Finally we selected BCL2-like 11 (BCL2L11) and BCL-2-modifying factor (BMF) as two anti-apoptotic proteins. All predicted targets harbor 1 3'UTR 8-mer conserved binding site, except for ETS1, which has a 7-mer conserved binding site and an additional 8-mer non-conserved binding site (Supplementary Figure 7A). Luciferase reporter assays revealed that the $3^{\prime} U T R$ s of all putative targets except for CD4 were functional, as evidenced by suppression of the firefly luciferase signal by overexpression of miR-221-222 ( 20-fold, data not shown) but not by overexpression of a control sequence or the unrelated miR-29b (Figure 6). Moreover, mutation of three single bases within the binding site for the miR-221-222 seed abrogated luciferase suppression by premiR-221-222 in all targets. For ETS1, both sites were functional as only double, but not single binding site mutation could rescue the reporter signal from miR-221222 repression (Supplementary Figure 7B). To see whether these confirmed miR-221-222 targets were derepressed at the mRNA level by in vivo miR-221-222 inhibition, we measured their cardiac transcript levels using qRT-PCR at 7 days of VM in animals treated with miR221-222 or control antagomirs. Levels of CXCL12, BCL2L11 and TOX were significantly increased by miR-221-222 inhibition, while ETS1, ETS2, IRF2 and BCL2L11 were increased by trend (Supplementary Figure 4D).

\section{MiR-221-222 overexpression suppresses viral replication in but not viability of cardiomyocytes in vitro}

To assess whether targeting of inflammatory and apoptotic genes by miR-221-222 affects these processes in vitro, we overexpressed or inhibited miR-221 and miR-222 in cardiomyocytes, endothelial cells and fibroblasts. Transfection of premiR-221 and premiR-222 increased the levels of mature miR-221-222 in RCMs, while levels of the highly myocyte-expressed miR-133a were unaffected (Figure 7A and Supplementary Figure 8A). In agreement with the increased viral loads after antagomir-221-222 treatment in vivo, overexpression of miR-221-222 markedly and significantly suppressed viral replication, compared to control premiRs (5-fold after 48 hours, $p<0.05)$ (Figure 7A). PremiR-221-222 treatment of RCMs did not efficiently activate interferon type I expression through IRF2 repression, as transcript levels of interferon-beta (IFN- $\beta$ ) were markedly higher in control RCMs, consistent with the higher viral loads in these cells. In line with the suppressive effects of miR-221-222 overexpression on CVB3 replication, inhibition of miR-221-222 resulted in higher viral loads in RCMs after 72 hours of infection (Figure 7B and Supplementary Figure 8B). We observed no differences in myocyte viability after 120 hours of infection (Figure 7A), in agreement with the lack of effect on cardiac apoptosis observed in vivo after miR-221-222 antagomir treatment. Transcript levels of direct target ETS2 was not affected by premiR-221-222, while direct targets CXCL12, ETS1, and IRF2, as well as the chemokine MCP1 were by trend repressed by premiR-221-222 

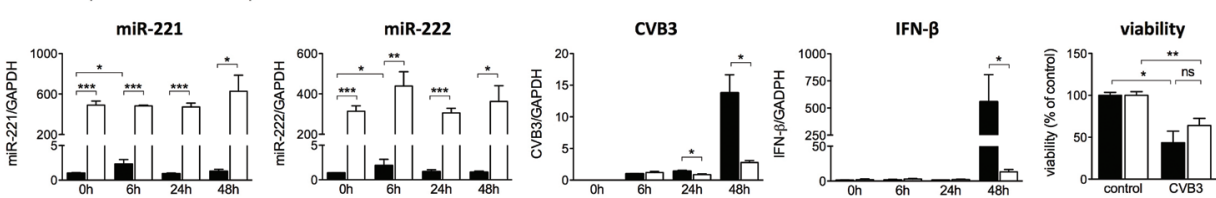

b
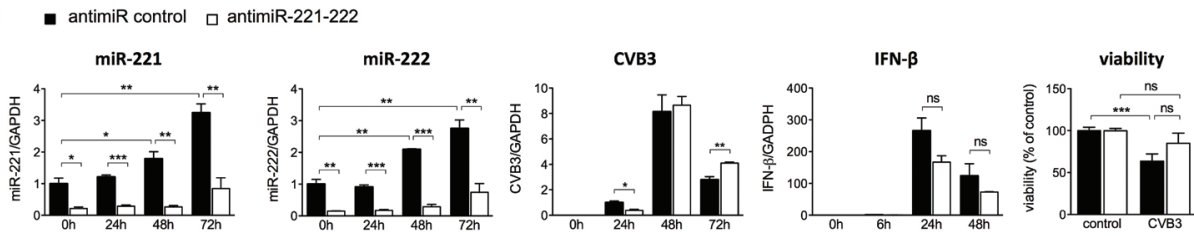

Figure 7. MiR-221-222 overexpression suppresses viral replication but not viability in cardiomyocytes in vitro. RCMs were transfected with mimics for (A) or inhibitors (B) against miR-221-222 or control and infected with CVB3 $(\mathrm{MOI}=1)$. Mimics and inhibitors efficiently overexpressed and knocked-down miR-221222, respectively. Overexpression of miR-221-222 significantly suppressed CVB3 replication and IFN- $\beta$ expression at 24 and 48 hours after infection ( $p<0.05$ for both). Conversely, inhibition of miR-221-222 caused higher CVB3 loads at 72 hours after infection $(p<0.01)$. Viability was assayed after 120 hours and was not affected by miR-221-222 overexpression or knockdown. ( $n \geq 3$ per group). ${ }^{*} P<0.05$, ${ }^{*} P<0.01$, *** $P<0.001$.

(Supplementary Figure 8A). Finally, overexpression or inhibition of miR-221-222 in endothelial cells (MCEC) did not reveal significant effects on MCEC cytokine or adhesion molecule expression to TNF- $\alpha$ stimulation (Supplementary Figure 9), and similarly overexpression of miR-221-222 in fibroblast did not suppress cytokine expression and interferon responses to TNF- $\alpha$ (Supplementary Figure 10). Together, these in vitro experiments indicate a potential role for miR-221-222 in CVB3 replication, while not supporting a role in cardiomyocyte apoptosis, endothelial cell adhesion molecule expression, or fibroblast interferon responses.

\section{Discussion}

In this study, we investigated the functional effects of miR-221-222 expression in the heart during VM in mice. We report that miR-221-222 levels are elevated in mouse VM but depressed in human VM, and that systemic inhibition of miR-221-222 in mice increases viral replication, cardiac injury and inflammation. These data add to our understanding of the role of miRNAs in VM and identify the miR-221-222 family as a protective factor in VM pathophysiology.

A striking finding from this study is the increased susceptibility of mice to VM-induced cardiac damage following miR-221-222 inhibition, suggesting that miR-221-222 upregulation is a compensatory molecular response during VM aiming to prevent virus-induced cardiac injury. In contrast, we previously reported that miR-155, which is also strongly upregulated during VM, contributes to cardiac inflammation, and that inhibition of miR-155 during VM 
in mice is protective against cardiac injury. Our data show that inhibition of miR-221-222 directly increases viral loads in vivo, both at day 4 and 7 post-infection and causes an early increased expression of the chemokine MCP1, that is produced by myocytes in response to CVB3 infection ${ }^{29}$. This mechanism was supported by miR-221-222 overexpression in cardiomyocytes in vitro, which strongly reduced CVB3 replication. It is not clear what direct miR-221-222 targets are responsible for these effects on CVB3 replication. ETS1 has been shown to promote the replication of HIV1 and HTLV1 (which are both single stranded RNA viruses like CVB3) in lymphoid cells ${ }^{25-27}$. It is possible that ETS1 may also affect CVB3 replication in cardiomyocytes, or additionally in lymphoid cells. Studies are underway to investigate these possibilities.

We identified a number of direct targets that suggest roles beyond viral replication for miR-221-222 in VM, including apoptosis (BCL2L11 and BMF), adhesion molecule expression (ETS1 and ETS2), cytokine secretion (ETS1 and ETS2, CXCL12), and T cell biology (TOX and CD4). Although we show that the pro-apoptotic factors BCL2L11 and BMF are bona fide targets of miR-221-222, miR-221-222 effects on viral load were not paralleled by differences in apoptosis, either in vitro or in vivo. It is well possible that the anti-apoptotic effects of miR-221-222 are cell type-dependent, as their expression has been reported to be anti-apoptotic or pro-apoptotic in different cellular contexts (hepatocytes and hepatocellular carcinoma cells, respectively $)^{30,31}$. In addition to targeting apoptotic genes, miR-221-222 directly target ETS1, which was previously shown to affect endothelial cell adhesion molecule expression and T cell adhesion in human umbilical vein endothelial cells $^{32}$. Although we did not observe adhesion molecule upregulation in murine endothelial cells upon miR-221-222 manipulation, the biological activity of ICAM1 and VCAM molecules is at least as much determined by cellular localization as by expression levels, and our data do not exclude that cellular adhesion may be promoted in MCEC cells independent of adhesion molecule transcript abundance.

We inhibited miR-221 and miR-222 expression levels in parallel in vivo, as we anticipated redundancy in target repression. Although the predicted target set of both miRNAs is highly overlapping (depending on the target prediction algorithm used), minor differences between their biological functions cannot be excluded from our experiments. Conversely, potential therapeutic strategies aimed at increasing miR-221 or miR-222 expression may not require overexpression of both family members. Additional experiments will be required to assess whether overexpression of both miRNAs is protective in vivo, and whether both family members possess differential efficacy for such purposes.

We observed divergent miR-221-222 expression response during VM in humans and mice. Previous studies reported downregulation of miR-221-222 in human ischemic and dilated cardiomyopathy ${ }^{18,19}$, while miR-221-222 upregulation in heterogenous acute murine heart disease ${ }^{33-36}$. The difference between mouse and human miR-221-222 expression during VM may thus reflect divergent transcriptional control of miR-221-222 expression, or may alternatively be caused by the fact that biopsies in human myocarditis are taken at a later time point after initial viral infection. Importantly, expression differences between species do not automatically imply differences in the cellular effects of miR-221-222 signaling between humans and mice. Rather, given the large number of direct and indirect targets 
affected by single miRNAs and the marked conservation of miR-221 and miR-222 throughout evolution, comparable biological functions in humans and mice are expected. Therefore, our identification of miR-221-222 as protective in murine VM may have direct bearing on human disease, suggesting that promoting miR-221-222 signaling may benefit VM patients.

In conclusion, miR-221 and miR-222 dysregulated in VM in humans and mice and miR-221222 inhibition in mice during VM leads to increased viral replication, cardiac injury and inflammatory infiltration. These data identify the miR-221-222 family as a protective component of the cardiac molecular response to CVB3 infection and as a potential therapeutic target in VM. 


\section{References}

1. Cooper LT, Jr. Myocarditis. N Engl J Med. 2009;360:1526-1538

2. Corsten MF, Schroen B, Heymans S. Inflammation in viral myocarditis: Friend or foe? Trends Mol Med. 2012;In press:1-12

3. Blauwet LA, Cooper LT. Myocarditis. Progress in cardiovascular diseases. 2010;52:274-288

4. Esfandiarei M, McManus BM. Molecular biology and pathogenesis of viral myocarditis. Annual review of pathology. 2008;3:127-155

5. Mason JW, O'Connell JB, Herskowitz A, Rose NR, McManus BM, Billingham ME, Moon TE. A clinical trial of immunosuppressive therapy for myocarditis. The myocarditis treatment trial investigators. The New England journal of medicine. 1995;333:269-275

6. Bartel DP. Micrornas: Target recognition and regulatory functions. Cell. 2009;136:215-233

7. Small EM, Olson EN. Pervasive roles of micrornas in cardiovascular biology. Nature. 2011;469:336-342

8. O'Connell RM, Rao DS, Chaudhuri AA, Baltimore D. Physiological and pathological roles for micrornas in the immune system. Nature reviews. Immunology. 2010;10:111-122

9. Pineau P, Volinia S, McJunkin K, Marchio A, Battiston C, Terris B, Mazzaferro V, Lowe SW, Croce CM, Dejean A. Mir-221 overexpression contributes to liver tumorigenesis. Proceedings of the National Academy of Sciences of the United States of America. 2010;107:264-269

10. Medina R, Zaidi SK, Liu CG, Stein JL, van Wijnen AJ, Croce CM, Stein GS. Micrornas 221 and 222 bypass quiescence and compromise cell survival. Cancer research. 2008;68:2773-2780

11. Zampetaki A, Mayr M. Micrornas in vascular and metabolic disease. Circulation research. 2012;110:508-522

12. Liu $X$, Cheng $Y$, Zhang S, Lin $Y$, Yang J, Zhang C. A necessary role of mir-221 and mir-222 in vascular smooth muscle cell proliferation and neointimal hyperplasia. Circulation research. 2009;104:476-487

13. Poliseno L, Tuccoli A, Mariani L, Evangelista M, Citti L, Woods K, Mercatanti A, Hammond S, Rainaldi G. Micrornas modulate the angiogenic properties of huvecs. Blood. 2006;108:3068-3071

14. Jorgensen S, Baker A, Moller S, Nielsen BS. Robust one-day in situ hybridization protocol for detection of micrornas in paraffin samples using Ina probes. Methods. 2010;52:375-381

15. Nuovo GJ. In situ detection of micrornas in paraffin embedded, formalin fixed tissues and the co-localization of their putative targets. Methods. 2010;52:307-315

16. De Windt LJ, Willemsen PH, Popping S, Van der Vusse GJ, Reneman RS, Van Bilsen M. Cloning and cellular distribution of a group ii phospholipase a2 expressed in the heart. J Mol Cell Cardiol. 1997;29:2095-2106

17. Schroen B, Heymans S. Micrornas and beyond: The heart reveals its treasures. Hypertension. 2009;54:11891194

18. Ikeda S, Kong SW, Lu J, Bisping E, Zhang H, Allen PD, Golub TR, Pieske B, Pu WT. Altered microrna expression in human heart disease. Physiol Genomics. 2007;31:367-373

19. Sucharov C, Bristow MR, Port JD. Mirna expression in the failing human heart: Functional correlates. J Mol Cell Cardiol. 2008;45:185-192

20. Suarez Y, Wang C, Manes TD, Pober JS. Cutting edge: Tnf-induced micrornas regulate tnf-induced expression of e-selectin and intercellular adhesion molecule-1 on human endothelial cells: Feedback control of inflammation. Journal of immunology. 2010;184:21-25

21. Graff JW, Dickson AM, Clay G, McCaffrey AP, Wilson ME. Identifying functional micrornas in macrophages with polarized phenotypes. The Journal of biological chemistry. 2012

22. Zhan Y, Brown C, Maynard E, Anshelevich A, Ni W, Ho IC, Oettgen P. Ets-1 is a critical regulator of ang iimediated vascular inflammation and remodeling. The Journal of clinical investigation. 2005;115:2508-2516 
23. Russell L, Garrett-Sinha LA. Transcription factor ets-1 in cytokine and chemokine gene regulation. Cytokine. 2010;51:217-226

24. Cheng C, Tempel D, Den Dekker WK, Haasdijk R, Chrifi I, Bos FL, Wagtmans K, van de Kamp EH, Blonden L, Biessen EA, Moll F, Pasterkamp G, Serruys PW, Schulte-Merker S, Duckers HJ. Ets2 determines the inflammatory state of endothelial cells in advanced atherosclerotic lesions. Circulation research. 2011;109:382-395

25. Sieweke $M H$, Tekotte $H$, Jarosch $U$, Graf T. Cooperative interaction of ets-1 with usf-1 required for hiv-1 enhancer activity in t cells. The EMBO journal. 1998;17:1728-1739

26. Posada R, Pettoello-Mantovani M, Sieweke M, Graf T, Goldstein H. Suppression of hiv type 1 replication by a dominant-negative ets-1 mutant. AIDS research and human retroviruses. 2000;16:1981-1989

27. Bosselut R, Duvall JF, Gegonne A, Bailly M, Hemar A, Brady J, Ghysdael J. The product of the c-ets-1 protooncogene and the related ets2 protein act as transcriptional activators of the long terminal repeat of human $t$ cell leukemia virus htlv-1. The EMBO journal. 1990;9:3137-3144

28. Kuhl U, Pauschinger M, Schwimmbeck PL, Seeberg B, Lober C, Noutsias M, Poller W, Schultheiss HP. Interferon-beta treatment eliminates cardiotropic viruses and improves left ventricular function in patients with myocardial persistence of viral genomes and left ventricular dysfunction. Circulation. 2003;107:27932798

29. Shen $Y, X u$ W, Chu YW, Wang Y, Liu QS, Xiong SD. Coxsackievirus group b type 3 infection upregulates expression of monocyte chemoattractant protein 1 in cardiac myocytes, which leads to enhanced migration of mononuclear cells in viral myocarditis. Journal of virology. 2004;78:12548-12556

30. Sharma AD, Narain N, Handel EM, Iken M, Singhal N, Cathomen T, Manns MP, Scholer HR, Ott M, Cantz T. Microrna-221 regulates fas-induced fulminant liver failure. Hepatology. 2011;53:1651-1661

31. Dai R, Li J, Liu Y, Yan D, Chen S, Duan C, Liu X, He T, Li H. Mir-221/222 suppression protects against endoplasmic reticulum stress-induced apoptosis via p27(kip1)- and mek/erk-mediated cell cycle regulation. Biological chemistry. 2010;391:791-801

32. Zhu N, Zhang D, Chen S, Liu X, Lin L, Huang X, Guo Z, Liu J, Wang Y, Yuan W, Qin Y. Endothelial enriched micrornas regulate angiotensin ii-induced endothelial inflammation and migration. Atherosclerosis. $2011 ; 215: 286-293$

33. Sayed D, Hong C, Chen IY, Lypowy J, Abdellatif M. Micrornas play an essential role in the development of cardiac hypertrophy. Circ Res. 2007;100:416-424

34. Tatsuguchi M, Seok HY, Callis TE, Thomson JM, Chen JF, Newman M, Rojas M, Hammond SM, Wang DZ. Expression of micrornas is dynamically regulated during cardiomyocyte hypertrophy. J Mol Cell Cardiol. 2007;42:1137-1141

35. Roy S, Khanna S, Hussain SR, Biswas S, Azad A, Rink C, Gnyawali S, Shilo S, Nuovo GJ, Sen CK. Microrna expression in response to murine myocardial infarction: Mir-21 regulates fibroblast metalloprotease-2 via phosphatase and tensin homologue. Cardiovasc Res. 2009;82:21-29

36. van Rooij E, Sutherland LB, Thatcher JE, DiMaio JM, Naseem RH, Marshall WS, Hill JA, Olson EN. Dysregulation of micrornas after myocardial infarction reveals a role of mir-29 in cardiac fibrosis. Proc Natl Acad Sci U S A. 2008;105:13027-13032 


\section{Supplementary Figures and Tables}

\begin{tabular}{lll} 
Species & miR-221 (3p) & miR-222(3p) \\
\cline { 2 - 2 } & agcuacauugucugcuggguuuc & agcuacaucuggcuacugggu \\
rhesus monkey agcuacauugucugcuggguuuc & agcuacaucuggcuacugggu \\
mouse & agcuacauugucugcuggguuuc & agcuacaucuggcuacugggu \\
rat & agcuacauugucugcuggguuuc & agcuacaucuggcuacugggu \\
chicken & agcuacauugucugcuggguuuc & agcuacaucuggcuacugggucuc \\
zebrafish & agcuacauugucugcuggguuuc & agcuacaucuggcuacugggucuc \\
nematode & non existent & non existent
\end{tabular}

b

Supplementary Figure 1. Sequence conservation and genomic context of miR-221 and miR-222. (A) The mature sequences of miR-221 and miR-222 have been evolutionary conserved back to zebrafish. (B) UCSC Genome browser screen shot. MiR-221 and miR-222 are located 850 bases apart and co-transcribed from a large intergenic region on the X-chromosome. Regulatory elements for miR-221-222 transcription as indicated by H3K27AC regulatory element footprints and DNAsel hypersensitivity clusters - are located over a region spanning from 80 kilobases $(\mathrm{kb})$ upstream to $50 \mathrm{~kb}$ downstream of the miR-222 transcription start site. Sequence conservation in this region is imperfect, which may explain differential activation of miR-221 and miR-222 expression between different species. 
$\square$ control $\square \mathrm{MOI}=1 \quad \square \mathrm{MOI}=5$

a $\mathrm{nRCM}+\mathrm{CVB3}$
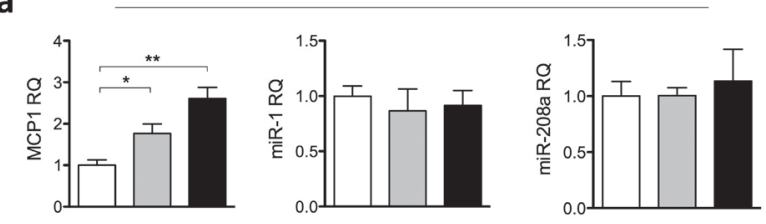

$\square$ control

- $24 \mathrm{~h}$

b

$\mathrm{nRCM}+\mathrm{TNF} \alpha$

$n R C F+T N F \alpha$

$\mathrm{MCEC}+\mathrm{TNF} \alpha$

BMM + LPS

T cells + ConA


Supplementary Figure 2. Control measurements for in vitro inflammatory stimulation experiments.

(A) CVB3 infection in rat neonatal myocytes (RCM) leads to the upregulation of macrophage chemoattractant protein-1 (MCP1), while the expression of the highly myocyte-expressed miR-1 and miR-208a is not affected. (B) Positive control genes confirming cellular expression responses to CVB3 infection or inflammatory stimulation in a cell panel including RCMs, fibroblasts (RCFs), adult mouse cardiac endothelial cells (MCEC), primary bone marrow-derived macrophages (BMM) and splenic T cells. Inflammatory stimuli were TNF- $\alpha$ for RCMs, RCFs and MCECs, lipopolysaccharide (LPS) for BMMs and Concanavalin A (ConA) for T cells ( $n=3$ per group). Expression levels are presented as relative to control. ${ }^{*} P<0.05,{ }^{*} P<0.01,{ }^{*}{ }^{*} P<0.001$. 
$\square$ control $\square 6 \mathrm{~h} \quad \mathbf{2} 24 \mathrm{~h}$

a

$\mathrm{nRCM}+\mathrm{II}-1 \beta$

$\mathrm{nRCF}+\| \mathrm{I}-1 \beta$
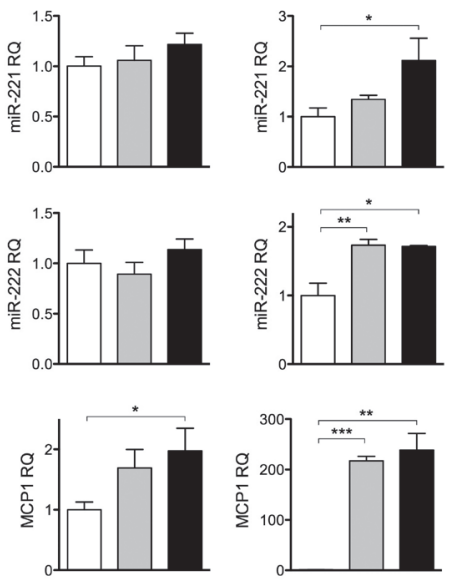

$\mathrm{MCEC}+\| \mathbf{I} \beta$
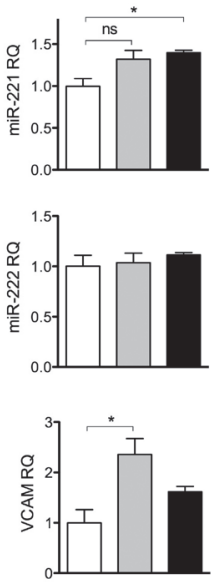

b

$3 T 3+$ TNF $\alpha$
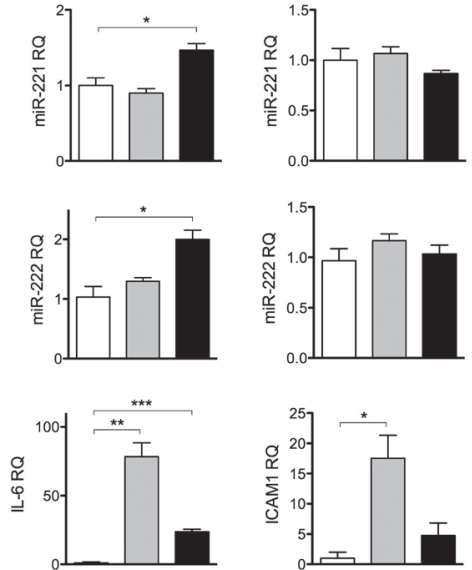

SVEC+ TNF $\alpha$
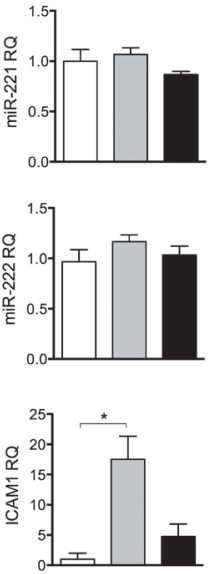

Supplementary Figure 3. MiR-221 and miR-222 expression responses to IL-1 $\beta$ and TNF- $\alpha$ in cardiac cells in vitro. (A) MiR-221 and miR-222 expression in response to stimulation with IL-1 $\beta$ in rat neonatal myocytes (RCM), fibroblasts (RCF) and adult mouse cardiac endothelial cells (MCEC). (B) MiR-221 and miR-222 expression in response to stimulation with TNF- $\alpha$ a mouse fibroblast cell line (3T3), and mouse endothelial cell line (SVEC). Expression levels are presented as relative to control. ${ }^{*} P<0.05,{ }^{*} P<0.01$, ${ }^{* *} P<0.001$ 
$\square$ PBS

a



$\square$ antagomir-221-222

b

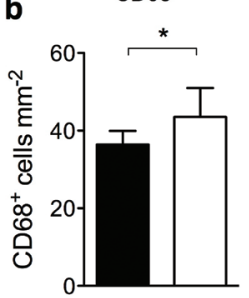

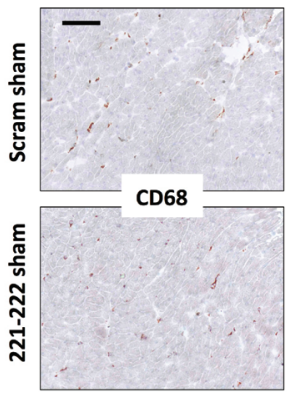

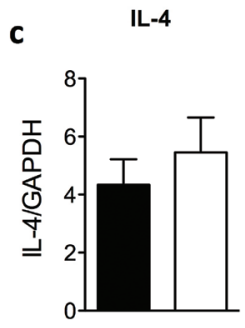

d

- antagomir control $\square$ antagomir-221-222

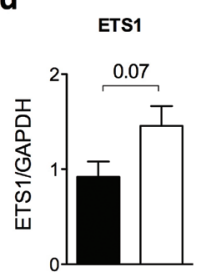

IRF2

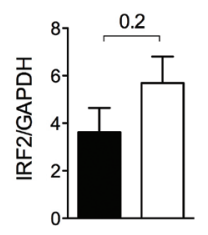

BCL2L11

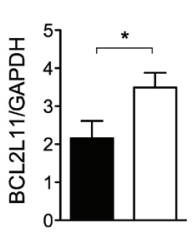

BMF

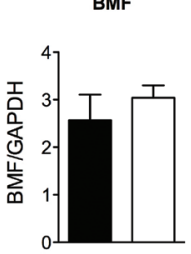

Supplementary Figure 4. Inhibition of miR-221-222 inflammation during acute VM in mice. (A) MiR221-222 inhibition increased white blood cell (WBC) counts in the systemic circulation. (B) The number of CD68+ macrophages in sham hearts was not affected by antagomir-221-222 administration. (C) Cardiac IL-4 transcript levels during VM were not affected by antagomir-221-222 administration. (D) Cardiac transcript levels of the direct miR-221-222 targets BCL2L11 and TOX were significantly increased by in vivo miR-221222 inhibition at VM day 7, while ETS1, ETS2 and IRF2 were increased by trend, and BMF was unaffected. Scale bars: $100 \mu \mathrm{m} .{ }^{*} \mathrm{P}<0.05$. 

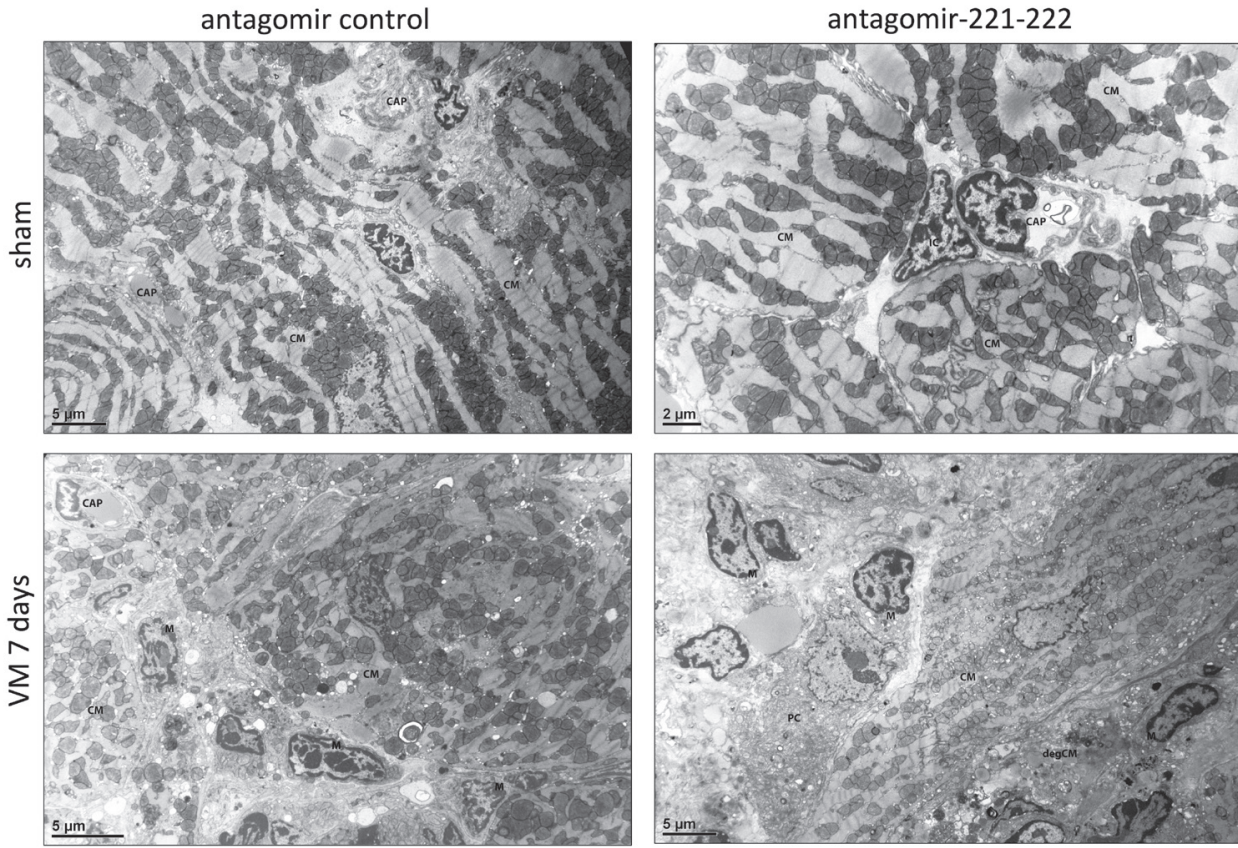

Supplementary Figure 5. Transmission electron microscopy (TEM) analysis of the mouse left ventricle in sham and VM animals treated with antagomir-221-222 or antagomir control. Normal ultrastructural morphology of cardiomyocytes (CM) and capillary blood vessels (CAP) is observed in hearts of sham animals treated with antagomir-221-222 or control. In control VM hearts, several mononuclear cells (M) have infiltrated in the interstitial space between the cardiomyocytes (CM). In the VM heart treated with antagomir-221-222, several mononuclear cells (M) and a plasma cell (PC) are seen around the cardiomyocyte (CM), as well as degenerating cardiomyocytes (degCM). IC: interstitial cell.

antagomir control

IL-1b

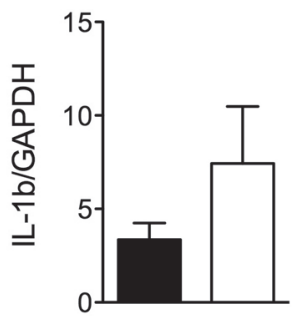

$\square$ antagomir-221-222

VCAM

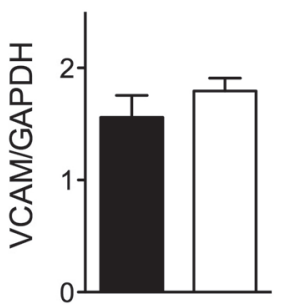

Supplementary Figure 6. Effects of miR-221-222 inhibition on cardiac transcript levels at day 4 post-infection in mouse VM hearts. Cardiac transcript levels of IL-1 $\beta$ were elevated by trend in antagomir-221-222 treated VM hearts at day 4, while VCAM levels were not affected by miR-221-222 inhibition. ( $n=5$ per sham group and $n=7$ per VM group). 


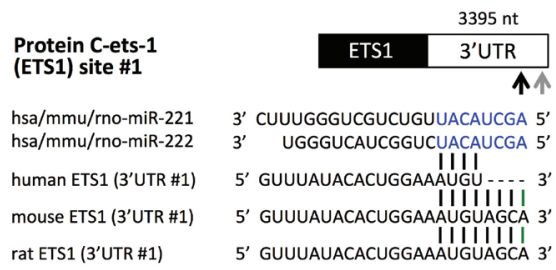

\section{Protein C-ets-1 (ETS1) site \#2}

$\mathrm{hsa} / \mathrm{mmu} / \mathrm{rno}-\mathrm{miR}-221$ hsa/mmu/rno-miR-222

human ETS1 (3'UTR)

mouse ETS1 (3'UTR)

rat ETS1 (3'UTR)

\section{Protein C-ets-2}

(ETS2)

$\mathrm{hsa} / \mathrm{mmu} / \mathrm{rno}-\mathrm{miR}-221$ $\mathrm{hsa} / \mathrm{mmu} /$ rno-miR-222

human ETS2 (3'UTR)

mouse ETS2 (3'UTR)

rat ETS2 ( 3 'UTR)

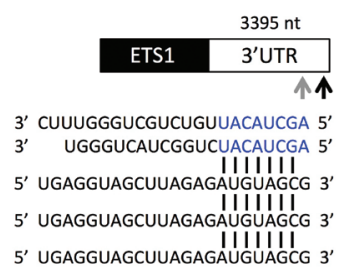

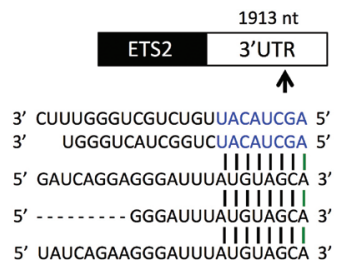

Interferon regulatory factor 2 (IRF2)

\section{IRF2}

$1236 n$

$\mathrm{hsa} / \mathrm{mmu} / \mathrm{rno}-\mathrm{miR}-221$

3' CUUUGgGUCGUCUGUUACAUCGA 5' hsa/mmu/rno-miR-222

human IRF2 (3'UTR)

mouse IRF2 ( $3^{\prime} U T R$ ) 3' UGGGUCAUCGGUCUACAUCGA 5 3 . IIIIIII agCUUUUAUGagCuCAUGUAGC 5' AACUUUUAUGAGCUCAUGUAGCA 3' - AACUUUUAUGAGUCAUGUGCA rat IRF2 (3'UTR) 5' AaCUUUUAUGAGCUCAUGUAGCA 3

Supplementary Figure 7. Conservation of miR-221-222 binding sites in the 3'UTR of inflammatory and anti-apoptotic protein targets.

(A) Schematic representation of the miR-221-222 seed sequence match with the 3'UTRs of 8 predicted miR-221-222 targets, including the pro-inflammatory transcription factors ETS1 and ETS2, the inhibitor of type I interferons transcription IRF2, the chemokine CXCL12, the anti-apoptotic proteins BCL2L11 and BMF, and the T cell factors TOX and CD4. The size of the 3'UTRs (mouse) is indicated above each target and arrowheads indicate the location of predicted binding sites within the $3^{\prime} U T R$. Watson-Crick complementarity in the seed region is indicated in black verticals, while an adenosine at the position across the first miRNA base (which outperform complementary bases for target prediction as described previously6) is indicated in green. (B) Luciferase experiments revealed that both predicted miR-221-222 binding sited in the ETS1 3'UTR are functional, as isolated mutation of either of the binding sites did not abrogate luciferase suppression by miR-221-222. ${ }^{* * P}<0.01$, *** $P<0.001$. 
Supplementary Figure 7 (continued)

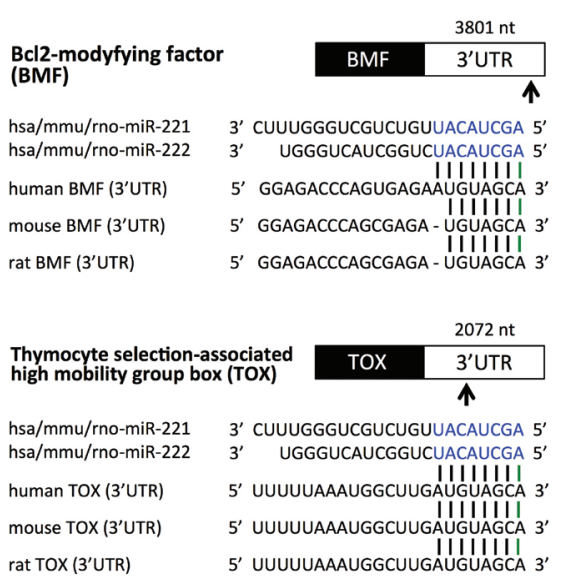

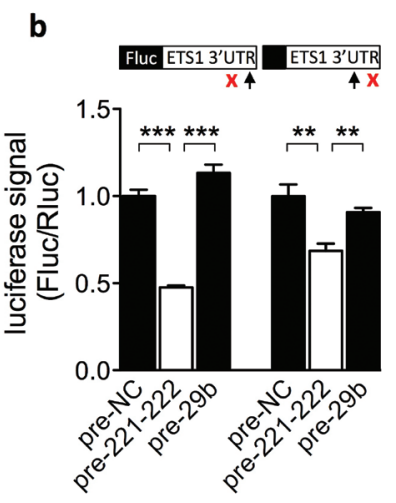

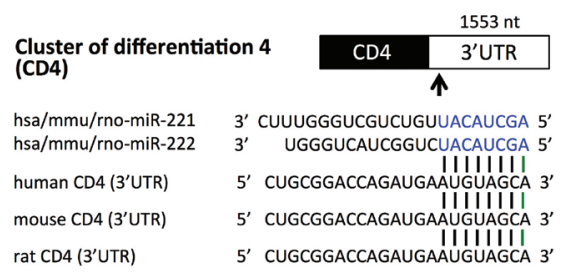


- premiR control a premiR-221-222
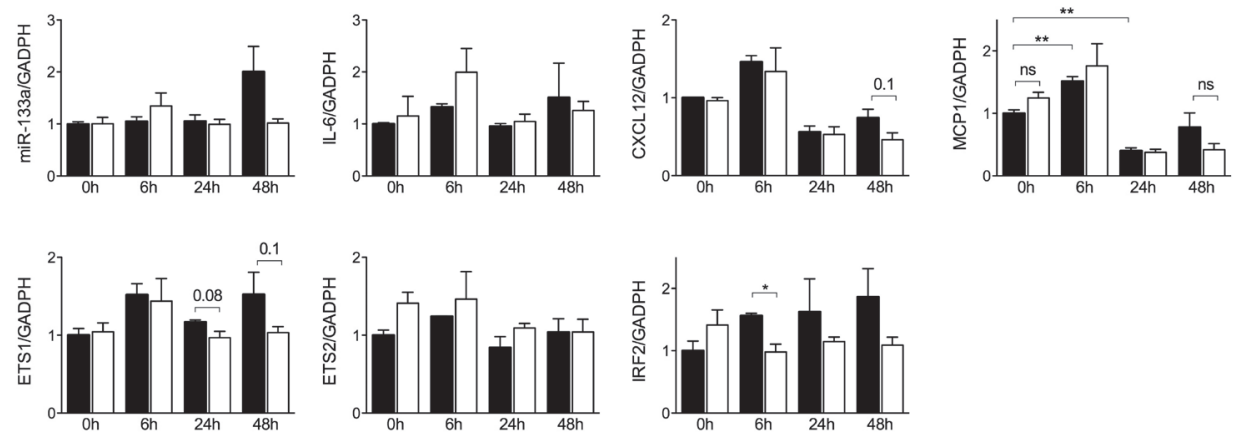

b

- antimiR control $\square$ antimiR-221-222

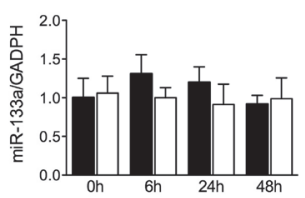

Supplementary Figure 8. Effects of miR-221-222 overexpression and inhibition in cardiomyocytes in vitro. Neonatal rat cardiomyocytes were transfected with mimics (A) or inhibitors (B) against miR-221-222 or control and infected with CVB3 $(\mathrm{MOI}=1)$. Mimics and inhibitors did not affect levels of miR-133a, and mimics did not significantly alter the expression levels of IL-6, CXCL12, MCP1, ETS1, ETS2 and IRF2. ${ }^{*} \mathrm{P}<$ $0.05,{ }^{*} P<0.01$. 
a
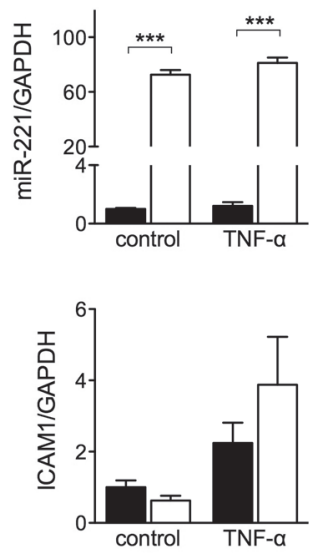

b
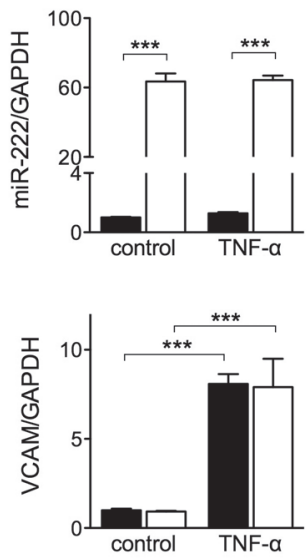
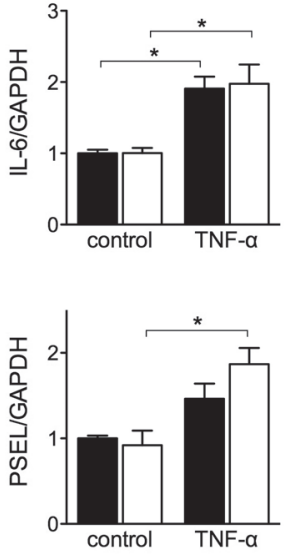
- antimiR control

$\square$ antimiR-221-222
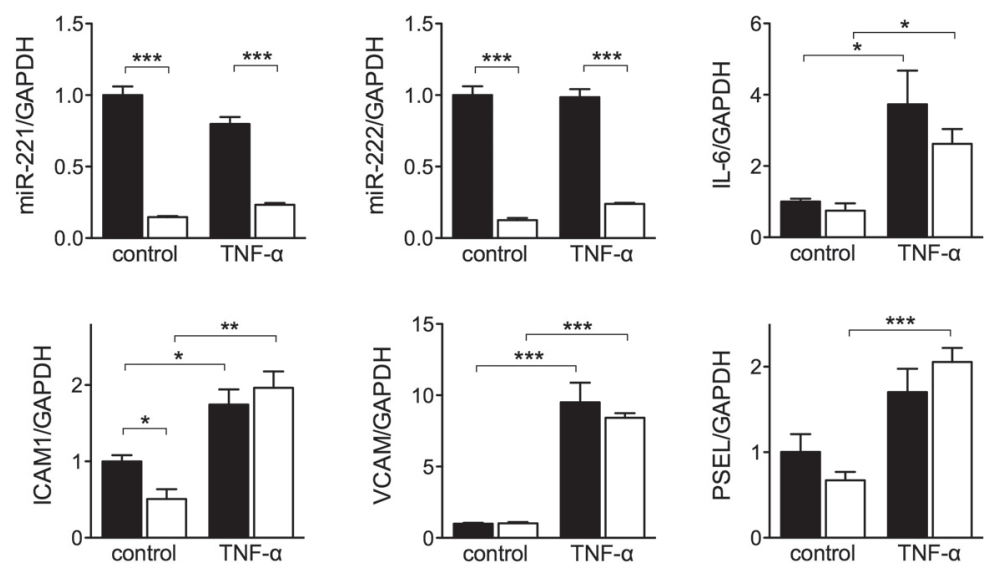

Supplementary Figure 9. Overexpression or inhibition of miR-221-222 do not affect TNF- $\alpha$-induced adhesion molecule expression in mouse cardiac endothelial cells (MCECs) in vitro. MCECs were transfected with mimics (A) or inhibitors (B) against miR221-222 or control and stimulated with TNF- $\alpha$ for 6 hours. Mimics and inhibitors efficiently overexpressed and knocked-down miR-221-222, respectively, but did not affect the induction of IL-6 or transcript levels of adhesion molecules ICAM1, VCAM and P-selectin in response to TNF- $\alpha$ stimulation. ( $n=4$ per group). ${ }^{*} P<0.05,{ }^{*} P<0.01,{ }^{*}{ }^{*} P<0.001$. 
- premiR control

$\square$ premiR-221-222
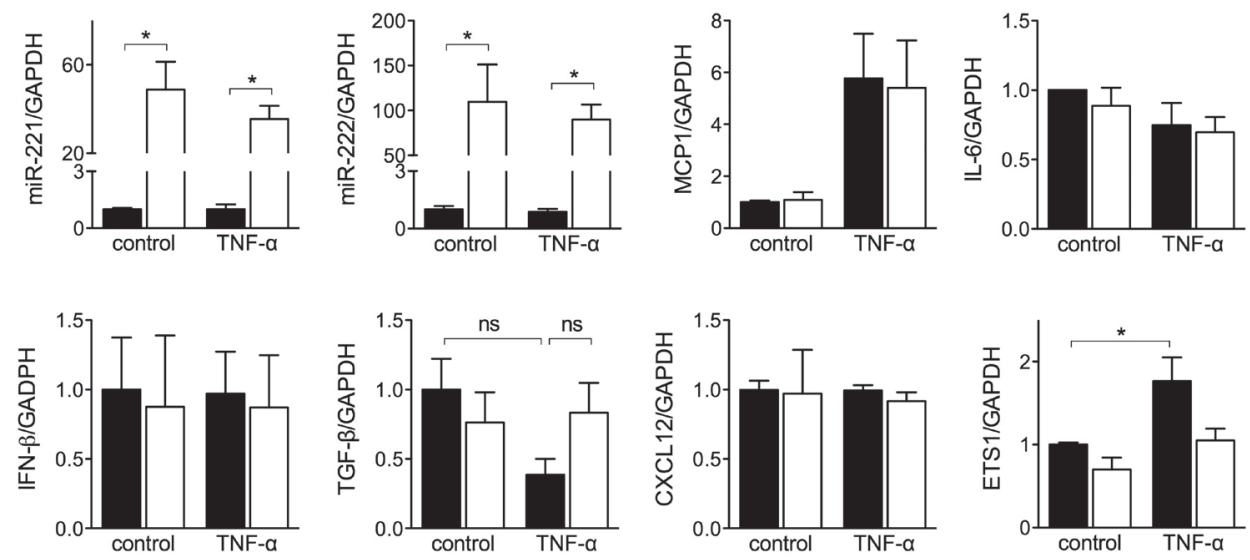

Supplementary Figure 10. Overexpression of miR-221-222 does not affect TNF- $\alpha$-induced cytokine responses in neonatal rat fibroblasts (RCFs) in vitro. MCECs were transfected with mimics against miR221-222 or control and stimulated with TNF- $\alpha$ for 24 hours. Mimics efficiently overexpressed miR-221-and miR-222, but did not significantly affect the transcripts levels of IL-6, MCP1, IFN- $\beta$, transforming growth factor-beta (TGF- $\beta$ ), CXCL12, or ETS1. ( $n=3$ per group). ${ }^{*} P<0.05$. 
Supplementary Table 1. Acute viral myocarditis and control sample donor characteristics

\begin{tabular}{|c|c|c|c|}
\hline Characteristic & $\begin{array}{l}\text { Viral Myocarditis } \\
\qquad(n=4)\end{array}$ & $\begin{array}{l}\text { Controls } \\
\qquad(n=6)\end{array}$ & $\mathbf{P}^{*}$ \\
\hline Age, years & $39 \pm 20$ & $38 \pm 11$ & 0.96 \\
\hline Women & $0(0)$ & 2 (33.3) & 0.52 \\
\hline \multicolumn{4}{|l|}{ Echocardiography } \\
\hline $\operatorname{LVEF}(\%)$ & $25 \pm 15$ & $55 \pm 7$ & 0.002 \\
\hline $\mathrm{EDD}(\mathrm{mm})$ & $48 \pm 10$ & $56 \pm 5$ & 0.15 \\
\hline PVB19 > threshold $\dagger$ & $4(100)$ & $0(0)$ & 0.005 \\
\hline PVB19 titer (c/ug DNA) & $1855 \pm 1508$ & $79 \pm 56$ & 0.04 \\
\hline CD45 cell/mm2 & $80 \pm 39$ & $11 \pm 4$ & 0.005 \\
\hline Serum Troponin T, $\mu \mathrm{g} / \mathrm{L}$ & $2.9 \pm 3.7$ & ND & \\
\hline
\end{tabular}

Data are presented as mean \pm SD, or $n(\%),{ }^{*}$ P-values based on student t-test, Mann-Whitney test or Chi-square analysis, † Threshold value: 500 copies/ $\mu$ g cardiac DNA, LVEF Left Ventricular Ejection, EDD End diastolic Diameter, ND Not determined

Supplementary Table 2. Primer sequences

\begin{tabular}{|c|c|c|}
\hline Gene & Forward primer & Reverse primer \\
\hline CVB3 & ACGAATCCCAGTGTGTTTTGG & TGCTCAAAAACGGTATGGACAT \\
\hline mmu-CXCL12 & AGAGCCAACGTCAAGCATCT & TAATTTCGGGTCAATGCACA \\
\hline mmu-GAPDH & GGTGGACCTCATGGCCTACA & СTCTCTTGCTCAGTGTCCTTGCT \\
\hline mmu-ICAM1 & TGGAGACGCAGAGGACCTTA & CGCTCAGAAGAACCACCTTC \\
\hline mmu-IFN- $\gamma$ & GCGTCATTGAATCACACCTG & CTGGACCTGTGGGTTGTTG \\
\hline mmu-IFN- $\beta 1$ & AGCACTGGGTGGAATGAGAC & TCCCACGTCAATCTTTCCTC \\
\hline mmu-interleukin-4 & CATGGGAAAACTCCATGCTT & TGGACTCATTCATGGTGCAG \\
\hline mmu-interleukin-6 & CAAAGCCAGAGTCCTTCAGAG & GCСАСТССTTCTGTGACTCC \\
\hline mmu-interleukin-10 & ATCGATTTCTCCCCTGTGAA & TGGCCTTGTAGACACCTTGG \\
\hline mmu-MCP1 & GTTGGCTCAGCCAGATGCA & AGCCTACTCATTGGGATCATCTTG \\
\hline mmu-P-selectin & TTCAGGACAATGGACAGCAG & GAAGGTGCAGGTTGATCCAT \\
\hline mmu-TNF- $\alpha$ & CCACCACGCTCTTCTGTCTA & AGGGTCTGGGCCATAGAACT \\
\hline mmu-VCAM & CCGGCATATACGAGTGTGAA & GATGCGCAGTAGAGTGCAAG \\
\hline rno-CXCL12 & GCCAACGTCAAACATCTGAA & TAATTTCGGGTCAATGCACA \\
\hline rno-ETS1 & GCTGTGGCAGTTTCTTCTGG & ТТССТСТТТССССАТСТССТ \\
\hline rno-ETS2 & CAGTAAGCCGACCATGTCCT & AGAAACTGCCACAGCTGGAT \\
\hline rno-IL-6 & GCCAGAGTCATTCAGAGCAA & GAGCATTGGAAGTTGGGGTA \\
\hline rno-IRF2 & CGAAACTACCGACAGTGTGC & CCCCATGTTGCTGAGGTACT \\
\hline rno-MCP1 & ATGCAGTTAATGCCCCACTC & TGCTGCTGGTGATTCTCTTG \\
\hline rno-GAPDH & GGTGGACCTCATGGCCTACA & СТСTCTTGСTCTCAGTATCCTTGCT \\
\hline
\end{tabular}


Supplementary Table 3. Cloning primer sequences

\begin{tabular}{|c|c|c|c|c|}
\hline Gene & $\begin{array}{c}\text { Seed } \\
\text { match }\end{array}$ & $\begin{array}{l}\text { Fragment } \\
\text { (nt) }\end{array}$ & $\begin{array}{l}\text { Forward } \\
\text { primer* }\end{array}$ & $\begin{array}{l}\text { Reverse } \\
\text { primer }^{\dagger}\end{array}$ \\
\hline mmu-ETS1 & $1 \times 8 \mathrm{mer}, 1 \times 7 \mathrm{mer}$ & 961 & АСTCCTCAGCGTGCTGACTT & TGTGCCAGCATCAGCTACTT \\
\hline mmu-ETS2 & $8 m e r$ & 1000 & TGAAGGGTGACCTGGTTTTT & GCTCCAAGTCCCATGTCTGT \\
\hline mmu-IRF2 & 8 mer & 749 & GGCTGCTTCTGCACCTTATC & TTCATTTATTATCAATCCACAGGA \\
\hline mmu-CXCL12 & $8 m e r$ & 832 & CAGGAGGAGCCTAGGTCTGA & ACCACCTTTCTTTGGGCTTT \\
\hline mmu-BCL2L11 & $8 \mathrm{mer}$ & 934 & ACCCATCAAACCCATTTTTG & TGAAAGCTAGTCGCAAGTTTTATTT \\
\hline mmu-BMF & 8 mer & 831 & TTCAAAGTTTGGCTGTGCTG & GGTCCCCATTCCTTTCCTTA \\
\hline mmu-TOX & 8 mer & 751 & TTTCACCATGGATTGCTTTG & AGTGAGCATTTGGCAGCTTT \\
\hline mmu-CD4 & 8mer & 957 & САCСTGCGTCCTGTCTCAT & CCATGTAGTGGCTTGGCTCT \\
\hline
\end{tabular}

* forward primers included a 5' Pme1-site (GTTTAAAC), † reverse primers included a 5'denotes 5' Nhe1-site (GCTAGC)

Supplementary Table 4. Mutation primer sequences

\begin{tabular}{ll}
\hline Gene & \multicolumn{1}{c}{ Mutation primer* } \\
\hline mmu-ETS1 site \#1 & GGTTTATACACTGGAAATGTCTAAGCATTGCTGCATTGATGCT \\
mmu-ETS1 site \#2 & CTGAGGTAGCTTAGAGATGTCTAGATGTAAGTGTCTTAAATGT \\
mmu-ETS2 & GGAAATATATGGGATTTATGTCTAAGCTATTTTTACTGCAAAAG \\
mmu-IRF2 & CTTTTATGAGCTCATGTCTAAATCAAATTATCCTGTGGATTG \\
mmu-CXCL12 & GCTGTACTTTCACATTTTATTATGTCTAAAGATACATGGTGATTT \\
mmu-BCL2L11 & GTCAGAACCCAGCGTATGTCTAATTTGTATTGCAGTTTCCC \\
mmu-BMF & GGGAGACCCAGCGAGATGTCTAATTTCCTTCATCCCCAGTC \\
mmu-TOX & GACTTTTTAAATGGCTTGATGTCTAAGTCATAGCAAGTTTGTAA \\
\hline
\end{tabular}

* forward primers are shown, reverse primers were the perfect reverse complement of the forward primers. The seed region is in bold, the mutate bases underlined (original seed: ATGTAGCA, mutated seed ATGTCTAA)

Supplementary Table 5. Biometrics of mice treated with antagomir-221-222 or antagomir control (VM day 7)

\begin{tabular}{lcccccc}
\hline & $\begin{array}{c}\text { PBS } \\
\text { sham }\end{array}$ & $\begin{array}{c}\text { anti-ctrl } \\
\text { sham }\end{array}$ & $\begin{array}{c}\text { anti-221-2 } \\
\text { sham }\end{array}$ & $\begin{array}{c}\text { PBS } \\
\text { VM }\end{array}$ & $\begin{array}{c}\text { anti-ctrl } \\
\text { VM }\end{array}$ & $\begin{array}{c}\text { anti-221-2 } \\
\text { VM }\end{array}$ \\
\hline Number & 11 & 5 & 11 & 20 & 10 & 20 \\
Mortality & 0 & 0 & 0 & 0 & 0 & 3 \\
Body weight baseline (g) & $20.0 \pm 0.5$ & $19.8 \pm 1.0$ & $20.0 \pm 0.6$ & $19.6 \pm 0.6$ & $19.8 \pm 0.5$ & $20.6 \pm 0.6$ \\
Body weight at sacrifice (g) & $24.6 \pm 0.6$ & $23.3 \pm 0.4$ & $22.4 \pm 0.5$ & $20.4 \pm 0.7$ & $21.8 \pm 1.0$ & $21.4 \pm 0.7$ \\
Heart weight / BW (mg/g) & $4.1 \pm 0.1$ & $3.9 \pm 0.2$ & $3.7 \pm 0.04$ & $4.6 \pm 0.1$ & $4.7 \pm 0.3$ & $5.6 \pm 0.3^{*}+\dagger \dagger$ \\
Lung weight / BW (mg/g) & $7.6 \pm 0.1$ & $7.8 \pm 0.1$ & $6.8 \pm 0.1$ & $6.8 \pm 0.1$ & $7.3 \pm 0.2$ & $7.3 \pm 0.3$ \\
Liver weight / BW (mg/g) & $79 \pm 1$ & $80 \pm 3$ & $76 \pm 1$ & $63 \pm 1 \dagger$ & $70 \pm 1+\dagger$ & $69 \pm 1 \dagger$ \\
Spleen weight / BW (mg/g) & $5.6 \pm 0.4$ & $6.8 \pm 0.5$ & $5.0 \pm 0.3$ & $6.5 \pm 0.4$ & $7.9 \pm 0.2$ & $8.3 \pm 0.5+\dagger \dagger$ \\
Kidney weight / BW (mg/g) & $8.9 \pm 0.2$ & $8.2 \pm 0.2$ & $7.9 \pm 0.1$ & $7.5 \pm 0.2 \dagger$ & $7.4 \pm 0.2 \dagger$ & $7.3 \pm 0.2$ \\
\hline
\end{tabular}

Data are presented as mean \pm SEM; Organ weights were normalized for baseline body weights $(B W),{ }^{*} p<0.05$; ${ }^{*} p<0.01 ;{ }^{* *}<0.001$ versus antagomir control, $+p<0.05 ;+\uparrow p<0.01 ; \dagger+\uparrow p<0.001$ versus sham 
MicroRNA-221-222 signaling is protective in acute viral myocarditis

Supplementary Table 6. Biometrics of mice treated with antagomir-221-222 or antagomir control (VM day 4)

\begin{tabular}{lcccc}
\hline & $\begin{array}{c}\text { anti-control } \\
\text { sham }\end{array}$ & $\begin{array}{c}\text { anti-221-222 } \\
\text { sham }\end{array}$ & $\begin{array}{c}\text { anti-control } \\
\text { VM day 4 }\end{array}$ & $\begin{array}{c}\text { anti-221-222 } \\
\text { VM day 4 }\end{array}$ \\
\hline Number & 3 & 3 & 7 & 7 \\
Body weight baseline (g) & $20.4 \pm 1.0$ & $21.1 \pm 0.3$ & $21.3 \pm 0.3$ & $21.2 \pm 0.6$ \\
Body weight at sacrifice (g) & $21.6 \pm 1.1$ & $22.6 \pm 0.1$ & $21.0 \pm 0.6$ & $21.0 \pm 0.6$ \\
Heart weight / BW (mg/g) & $3.6 \pm 0.1$ & $3.6 \pm 0.1$ & $3.2 \pm 0.05 \dagger$ & $3.4 \pm 0.05$ \\
Liver weight / BW (mg/g) & $72 \pm 1$ & $74 \pm 2$ & $63 \pm 2$ & $61 \pm 2 \dagger$ \\
Spleen weight / BW (mg/g) & $5.8 \pm 0.5$ & $4.7 \pm 0.2$ & $6.1 \pm 0.3$ & $5.4 \pm 0.3$ \\
\hline
\end{tabular}

Data are presented as mean $\pm \mathrm{SEM}$; Organ weights were normalized for baseline body weights $(\mathrm{BW}),{ }^{*} \mathrm{p}<0.05$; ${ }^{*} p<0.01 ;{ }^{* *}<0.001$ versus antagomir control, $\uparrow p<0.05 ; \dagger \uparrow p<0.01 ; \dagger \uparrow \uparrow p<0.001$ versus sham 
MicroRNA-221-222 signaling is protective in acute viral myocarditis 


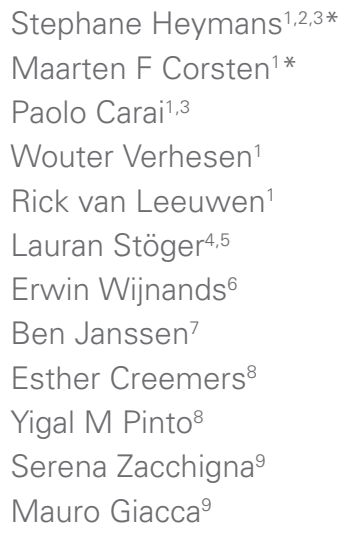

* Contributed equally
Dirk Grimm ${ }^{10}$

Nina Schürmann ${ }^{10}$

Elena Vigorito ${ }^{11}$

Thomas Thum ${ }^{12}$

Frank Stassen ${ }^{13}$

Peter Carmeliet ${ }^{14}$

Manuel Mayr ${ }^{15}$

Leon J de Windt ${ }^{16}$

Esther Lutgens $s^{5,6,17}$

Menno de Winther 4,5

Anna-Pia Papageorgiou ${ }^{1,3}$

Blanche Schroen ${ }^{1}$

1 Center for Heart Failure Research, Department of Cardiology, Cardiovascular Research Institute Maastricht (CARIM), Maastricht University, The Netherlands

2 Interuniversity Cardiology Institute of the Netherlands (ICIN), Utrecht, The Netherlands

3 Center for Molecular and Cardiovascular Biology (CMVB), KU Leuven, Leuven, Belgium

4 Department of Molecular Genetics, CARIM, Maastricht University, The Netherlands

5 Department of Medical Biochemistry, Academic Medical Center Amsterdam, Amsterdam, The Netherlands

6 Department of Pathology, CARIM, Maastricht University, The Netherlands

7 Department of Pharmacology, CARIM, Maastricht University, The Netherlands

8 Heart Failure Research Center, Academic Medical Center, Amsterdam, The Netherlands

9 International Centre for Genetic Engineering and Biotechnology (ICGEB), Padriciano, Trieste, Italy

10 Cluster of Excellence CellNetworks, Department of Infectious Diseases, Heidelberg University, Germany

11 Laboratory of Lymphocyte Signaling and Development, Babraham Institute, Cambridge, UK

12 Institute for Molecular and Translational Therapeutic Strategies, Hannover Medical School, Germany

13 Medical Microbiology, Maastricht University, The Netherlands

14 Laboratory of Angiogenesis and Neurovascular Link, Vesalius Research Center, Leuven, Belgium

15 King's BHF Centre, King's College London, UK 


\section{Macrophage MicroRNA-155 Controls Cardiac Hypertrophy and Failure}

Submitted

\section{ABSTRACT}

Hypertension triggers hypertrophic remodeling of the cardiac muscle and subsequent heart failure, representing one of the major challenges for cardiovascular research in the twenty-first century. While neurohormonal and myocyte signaling are well-established underlying mechanisms, the implication of the inflammatory system is increasingly gaining interest. Here, we show that genetic loss or pharmacological inhibition of the leukocyte-derived microRNA-155 in mice reduces cardiac inflammation, hypertrophy and dysfunction in pressure-overloaded hearts. The contribution of microRNA-155 expression in macrophages to myocyte growth was confirmed in vitro, where medium of microRNA-155 knockout macrophages did not allow the hypertrophic growth of cardiomyocytes. In vivo cardiac myocyte-specific microRNA-155 overexpression did not affect cardiac hypertrophy or dysfunction, whereas bone marrow transplantation from wild type into microRNA-155 knockout animals rescued the cardiac myocytes' hypertrophic response. These effects were at least partly mediated by the direct microRNA-155 target Suppressor of Cytokine Signaling 1 (Socs1), as Socs1 knockdown in microRNA-155 knockout macrophages rescued the hypertrophic response.

These findings reveal that the inhibition of the inflammatory cell-derived microRNA-155 prevents cardiac hypertrophy and dysfunction, and demonstrate a causative relation between inflammatory signaling and heart failure. 


\section{Introduction}

Cardiac hypertrophy and consequent heart failure in response to chronic hypertension represent one of the major health challenges in Western societies ${ }^{1}$. Over the past decade, inflammatory mediators have become well-recognized contributors to heart failure ${ }^{2-4}$. Conversely, the role of inflammatory cells during pressure overload-induced hypertrophy and heart failure is less well established despite the notion that Angiotensin II (Ang II), one of the major mediators of hypertension-induced target organ damage and hypertrophy, is a powerful promoter of inflammation5. In this study, we show that microRNA-155 (miR155) expression by macrophages contributes to cardiac hypertrophy and failure, through up-regulation of pro-inflammatory paracrine signaling.

MicroRNAs are small non-coding RNAs that inhibit gene expression of complementary target genes at the post-transcriptional level ${ }^{6}$. Whereas others have studied the implication of cardiac myocyte- or fibroblast-derived microRNAs in cardiac hypertrophy and failure ${ }^{7-10}$, inflammatory microRNAs have hitherto remained unaddressed in cardiac disease. MiR-155 is one of the first described inflammatory microRNAs, whose expression is induced rapidly in myeloid and lymphoid cells upon exposure to a variety of inflammatory stimuli ${ }^{11}$, and whose upregulation has been observed in a multitude of inflammatory diseases, including rheumatoid arthritis and multiple sclerosis ${ }^{12-15}$. MiR-155-deficient mice generate normal leukocyte populations under steady-state conditions, but they display defective B-cell responses upon immunization and resistance to $T$ cell-dependent experimental autoimmune encephalomyelitis ${ }^{16-18}$. Conversely, forced expression of miR-155 in the hematopoietic system dysbalances inflammation-driven granulocyte/monocyte expansion and causes a myeloproliferative disorder ${ }^{19}$, in line with its increased expression in human myeloid cancers $^{20}$. In light of these accounts, miR-155 is highly likely to be of functional importance in innate immunity. However, in vivo evidence to support this conclusion or for a role of miR-155 in heart disease is so far lacking.

The purpose of our study was to determine whether macrophage-derived miR-155 has a role in cardiac disease. We demonstrate that miR-155 is a mediator of cardiac hypertrophy and failure in response to pressure overload. Absence of miR-155 leads to decreased proinflammatory cytokine production through its de-repressed target Suppressor of cytokine signaling 1 (Socs1). Consequently, pro-inflammatory and pro-hypertrophic signaling through the downstream pathway Signal Transducer and Activator of Transcription 3 (Stat3) decreases both in cardiac myocytes and macrophages in the absence of miR-155. Our data demonstrate that local inflammation in the myocardium is driven by miR-155 and aggravates cardiac remodeling, and support a role for miR-155 in innate immunity.

\section{Methods}

\section{Mouse models}

All animal experiments were approved by the Committees for Animal Welfare of Maastricht and Leuven Universities, and performed adhering to Dutch and Belgian law. Ten to twelve 
weeks-old male miR-155 KO and WT C57BI/6J mice weighing 20-25 grams were used ${ }^{17}$. Angiotensin II (Ang II, $2.5 \mu \mathrm{g} / \mathrm{g} /$ day) was infused subcutaneously by Alzet osmotic minipump 2004 (Cupertino, CA) for 7 and 28 days. Echocardiography was performed with the Vevo770 (Visualsonics, Toronto, Canada) and probe RMV707B (15-45 MHz) at days 0 and 28. At day 21, blood pressure was monitored with tail cuff (CODA, Kent Scientific, Torrington, CT). At day 7 or 28 , mice were sacrificed and hearts were isolated for histological and molecular analyses. Total RNA was isolated with RNeasy mini kit (Qiagen, Valencia, CA) and SYBR Green quantitative PCR analysis was performed on a Bio-Rad iCycler (Hercules, CA) to determine Acta1, Nppa, Nppb, $I 16$ and Gapdh expression (primers in Supplementary Table 3). Cardiac protein extracts $(50 \mu \mathrm{g})$ were immunoblotted with antibodies to Agtr1 (Abcam, Cambridge, UK), Stat3 (C-20 Santa Cruz Biotechnology, Santa Cruz, CA), P-Stat3 (Tyr705, Cell Signaling Technology, Danvers, MA) and Gapdh (Fitzgerald, Acton, MA). At day 7 , peripheral blood was taken for FACS analysis as previously described ${ }^{44}$.

For bone marrow transplantation, eight weeks-old male miR-155 KO and WT mice received water supplemented with neomycin (100 mg/l) and polymyxin B sulfate $(60.000 \mathrm{U} / \mathrm{l})$. One day before transplantation, mice were irradiated with a lethal dose of 6 Gy röntgen. Bone marrow cells of male miR-155 WT and KO littermates were derived by flushing the femur and tibia, and cells $\left(10^{7}\right)$ were injected in the tail vein of each recipient. After eight weeks, mice were treated with Ang II for 28 days as described above.

Eight weeks-old male WT C57BI/6J mice were tail vein-injected on three consecutive days with a total of $10 \mathrm{mg} / \mathrm{kg}$ LNA-modified antimiR-155 or control antimiR (Ribotask, Denmark). On the last day of injection, Ang II administration started as described above for 28 days. RNA was isolated from hearts with the miRVana microRNA isolation kit (Ambion, Austin, TX) and the miScript PCR system (Qiagen) was used to determine miR-155 expression. Eight weeks-old male WT C57BI/6J mice were tail vein-injected with $2.5 \times 10^{11}$ viral genome particles of adeno-associated virus serotype 9 (AAV9) in 100ul PBS. AAV9-pre-hsa-miR-155 and -scrambled vectors, and AAV9-sponge-miR-155 and sponge-control vectors were produced at high-titer as previously described ${ }^{45,46}$. The AAV9-sponge-miR-155 vector contained 4 imperfect binding sites for mmu-miR-155 (acccctatcacgtcaagcattaa; imperfect binding sites in bold, while the AAV9-sponge-control vector contained 3 mutated binding sites for miR-122 (acaaacaccattgtcagattcga; mutations in seed region in bold). Rescue efficiency of the sponge-miR-155 construct was tested in a luciferase assay. HEK293T cells were co-transfected with 1) sponge-miR-155 or sponge-control vector, 2) psiCheck2 luciferase vector (Promega) containing an imperfect miR-155 target sequence (the same also contained in the sponge) in the Renilla luciferase 3'UTR, and 3) either mmu-premiR-155 in pCDM vector (System Biosciences, Mountain View, CA) or pBluescriptll control vector (Stratagene, Santa Clara, CA). In vivo AAV9-mediated gene transfer and CMV-driven expression of pre-miR-155, scrambled, sponge-miR-155 and sponge-control was allowed for 3 weeks. Then, mice were treated with Ang II as described above.

\section{Histology}

Paraffin-embedded $4 \mu \mathrm{m}$ LV sections were stained with hematoxylin-eosin (HE) and Picro Sirious red (SR) as described before ${ }^{47}$, or were immunohistochemically stained at RT using 
Horse Reddish Peroxidase/DAB with monoclonal rat anti-mouse CD45 Leukocyte Common Antigen Ly-5 (BD-Biosciences Pharmingen, San Diego, CA) and monoclonal mouse antihuman desmin (Dako Cytomation, Denmark). Left ventricular cryo-sections of $7 \mu \mathrm{m}$ were immunohistochemically stained at RT using Alkaline Phosphatase/Fast Red with polyclonal rat anti-mouse antibodies against CD68, CD3 and Nimp1 (kind gift from Dr Menno de Winther). Morphometric analyses were performed using a microscope (Leica DM2000; Leica, Wetzlar, Germany), camera (Leica DFC295 3Mpix CMOS color), and LAS Image Analysis and OWin morphometry software (Leica). The number of positive cells was measured per $\mathrm{mm}^{2}$.

In situ hybridization was preformed as described ${ }^{48,49}$. In brief, $12 \mu \mathrm{m}$ cryo-sections were prepared from $4 \%$ paraformaldehyde-fixed hearts from WT and KO Angll-treated animals. Sections were air-dried for $30 \mathrm{~min}$, incubated in $1.3 \mathrm{mg} / \mathrm{ml}$ Pepsin $/ 0.1 \mathrm{mM} \mathrm{HCl}$ solution for $20 \mathrm{~min}$ at room temperature, and incubated overnight at $37^{\circ} \mathrm{C}$ in $1 \mathrm{x}$ hybridization buffer (ENZO Life sciences, Farmingdale, NY) with 40nM mmu-miR-155 double DIG-labeled probe (Exiqon, Vedbaek, Denmark). The following day the sections were washed twice in 5xSSC, 1XSSC and 0.2xSSC at hybridization temperature, and then blocked in DIG blocking buffer (Roche, Basel, Switzerland) for 20min followed by a 45min incubation in 1:800 anti-DIG-AP, Fab fragments (Roche). Sections were washed $3 \times 5$ min with DIG wash buffer and incubated 3min in DIG detection buffer at RT. DIG signal was detected by using NBT/BCIP tablets (Roche) for 4hrs at 30C in darkness. Finally, sections were briefly washed in MQ, quickly dehydrated through an ethanol gradient and mounted with Entellan (EMS, Hatfield, PA).

\section{FACS}

Peripheral blood was analyzed using FACS Cantoll flow cytometer (Becton Dickenson, San Diego, CA). Erythrocytes were removed by hypotonic lysis with $\mathrm{NH} 4 \mathrm{Cl}$. Cells were first incubated with anti-CD16/32 (eBioscience, San Diego, CA) to block Fc receptor binding and stained with anti-CD3e-FITC, anti-CD8a-eFLUO450, anti-CD25-APC, anti-B220-PE-Cy7 (eBioscience) and anti-CD4-PerCp (BD-Biosciences Pharmingen). Foxp3-positive cells were detected with PE anti-mouse/rat Foxp3 Staining Set (eBioscience). Leukocytes were further incubated with anti-CD11b-PE-Cy7, anti-ly6G-PE and NK1.1 PerCp-Cy5.5 (BD-Biosciences Pharmingen) to detect monocytes (CD11b+ly6G-NK1.1-) and granulocytes (CD11b+ly6G+NK1.1-).

\section{In vitro experiments}

Rat ventricular cardiac myocytes (RCMs) and fibroblasts (RCFs) were isolated by enzymatic dissociation of 1- to 2-day-old neonatal rats, plated on gelatinized 6-well plates and cultured as described previously ${ }^{50}$. Per well, $5^{*} 10^{5} \mathrm{RCMs}$ were grown on low serum media and transfected with 35nM miR-155 antagomiR or scrambled control (Ambion). After 24 hours, cells were treated with 100nM endothelin-1 (Et-1, Sigma, St. Louis, MO) or control for 24 hours, after which RNA was isolated with miRVana (Ambion) and quantitative PCR was performed as described above.

Bone marrow macrophages (BMMs) were obtained and cultured from miR-155 KO and WT mice as described previously ${ }^{51,52}$. Cells were stimulated with 10ng/ml LPS (from E. 
coli 055.B5; Sigma) for 0, 1, 6 and 24 hours, or transfected with siSocs 1 or siControl (Ambion) by electroporation as previously described ${ }^{53}$. Then media were collected, residual cells removed by centrifugation for $10 \mathrm{~min}$ at $300 \mathrm{~g}$, and media were stored at $-80^{\circ} \mathrm{C}$. BMMs were harvested for protein extraction or RNA isolation with miRVana RNA isolation kit. SOCS1 knockdown was assessed by RT-PCR using forward primer CCTCCTCGTCCTCGTCTTC and reverse primer AAGGTGCGGAAGTGAGTGTC, yielding a 103 nucleotides RT-PCR product, with GAPDH as a loading control (FGGTGGACCTCATGGCCTACA; R-CTCTCTTGCTCAGTGTCCTTGCT; 82 nucleotides). BMM proteins were blotted for Socs1 (Cell Signaling Technology). Media were subjected to II6 ELISA (kindly provided by Dr. Frank Stassen).

$\mathrm{KO}$ and WT BMM-conditioned media were dialyzed in low serum RCM media using slidea-lyzer dialysis cassettes (1/100, 3.5kDa, Thermo Fisher Scientific, Waltham, MA). RCMs were incubated with dialyzed conditioned media for 24 hours, treated with endothelin-1 or PBS for 24 hours, and either stained with rhodamin-phalloidin (Molecular probes, Invitrogen, Paisley, UK) or processed for RNA extraction (RNeasy) and qPCR as described above.

\section{Proteomics}

BMM media were subjected to mass spectroscopic proteomics analyses as previously described ${ }^{54}$. In brief, BMM media were concentrated using Amicon 3kD MWCO spin columns (Millipore) and precipitated by 2D Clean-up kit (Bio-Rad). After that, protein samples were denatured with $2 X$ sample buffer (Invitrogen) at $97^{\circ} \mathrm{C}$ for $5 \mathrm{~min}$. and separated in 4\%-12\% NuPAGE Bis-Tris gels (Invitrogen). Gels were stained using Coomassie G-250 overnight and destained. Each lane was cut into 16 gel bands without any gap and tryptic digestion was performed using the ProGest robot (Genomic Solutions) with sequencing grade modified trypsin (Promega) overnight. Digested peptides were lyophilized and resuspended with $0.05 \%$ TFA then separated by nano-flow liquid chromatography on a

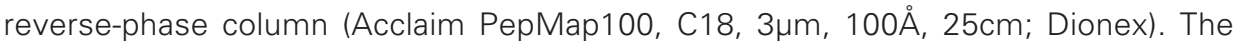
separated peptides were directly injected to a high mass accuracy ion trap tandem mass spectrometer (LTQ Orbitrap XL, Thermo). Spectra were collected from the mass analyzer using full ion scan mode over the $\mathrm{m} / \mathrm{z}$ range 450-1600. Six dependent MS/MS scans were performed using dynamic exclusion. The MS/MS data were matched to SwissProt mouse database (version 57.15, 16230 protein entries) using Mascot version 2.3.0 (Proteome Discoverer 1.1, Thermo Scientific). Carboxyamidomethylation of cysteine was used as a fixed modification, and oxidation of methionine was used as a variable modification. The mass tolerance was set at $10 \mathrm{ppm}$ for the precursor ions and $0.8 \mathrm{Da}$ for fragment ions. Two missed cleavage were allowed. Search results were loaded into Scaffold software (version 3.0.8, Proteome Software). Assignments were accepted with $>99.0 \%$ protein probability, $>95.0 \%$ peptide probability, and a minimum of two peptides.

\section{Statistical analyses}

Group numbers are given in Supplementary Table 1. Data are presented as average \pm SEM unless indicated otherwise. Comparisons between 2 groups were performed with two- 
tailed Student's t testing for Gaussian data or Mann-Whitney tests for non-Gaussian data. For comparisons of more than 2 groups, one-way ANOVA was used, followed by post hoc testing using Bonferroni correction for more groups. $\mathrm{P}<0.05$ was considered statistically significant.

\section{Results}

\section{Absence of miR-155 protects from heart failure following pressure overload}

We addressed the biological role of miR-155 in a pre-clinical model of heart failure, where we infused miR-155 KO and WT mice with a pressor-dose of Angiotensin II (Ang II) for 4 weeks. All mice survived the observation period. Whereas miR-155 WT mice developed impaired systolic function after Ang II, the absence of miR-155 protected against cardiac dysfunction (Figure 1A and Supplementary Table 1). Remarkably, miR-155 KO Ang II hearts showed dramatically decreased hypertrophy as compared to WT Ang II hearts (Figure 1B and C (upper panels)), despite similar blood pressure responses to Ang II (Figure 1D). This protection against cardiac hypertrophy was confirmed on the individual myocyte level (Figure 1C (lower panels), E) and by echocardiographically measured wall thickness (Figure 1F). Concordantly, transcript levels of cardiac stress markers alpha skeletal actin (Acta1), natriuretic peptide type A (Nppa) and natriuretic peptide type B (Nppb) remained low in Ang II treated miR-155 KO mice as compared to WT animals (Figure 1G). Capillary density was similar in untreated miR-155 KO and WT sham hearts (Figure 2A), and could therefore not explain differences in hypertrophy. In addition, as expected, vascularization increased with hypertrophy in WT mice. Interstitial fibrosis, quantified as percentage of Sirius red-stained area of the left ventricle, was increased in both WT and KO mice upon Ang II, indicating that Ang II-induced cardiac matrix deposition was unaffected by miR-155 deficiency (Figure 2b and Supplementary Table 1). Desmin staining as a marker of cardiac myocyte stress ${ }^{21,22}$ was dramatically reduced in LV sections of miR-155 KO Ang II compared to WT Ang II (Figure 2C). Cardiac protein levels of the angiotensin II type 1 receptor (Agtr1), which has been reported to be a miR-155 target in humans ${ }^{23}$ but is not conserved in mice, were not different between WT and KO mice (Figure 2D). Therefore, miR-155 KO mice are protected against Angll-induced cardiac hypertrophy and dysfunction by a mechanism that is independent of its target Agtr1, of blood pressure, of vascularization, and of cardiac fibrosis.

To confirm these findings in another model of pressure overload, we subjected miR-155 $\mathrm{KO}$ and WT mice to transverse aortic constriction (TAC). Again, miR-155 KO mice were protected from hypertrophy and heart failure (Figure 3A-D and Supplementary Table 1), and their induction of hypertrophic markers Acta1, Nppa and Nppb was blunted (Figure 3E). Both strains had trans-stenotic pressure gradients above $50 \mathrm{mmHg}$ on average (not shown) and interstitial fibrosis was not different between WT and KO mice after TAC (Figure 3F and Supplementary Table 1). Additionally, to ensure that the observed protection against heart failure was not due to differences inherent to embryologic development between miR-155 $\mathrm{KO}$ and WT mice, we injected LNA-modified antimiRs against miR-155 (antimiR-155) into 


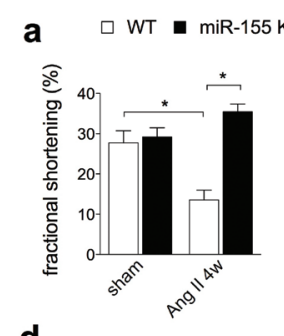

d

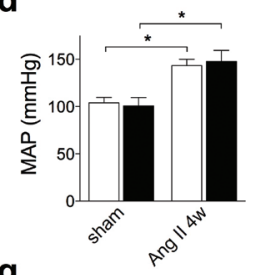

g

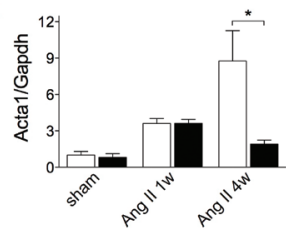

b

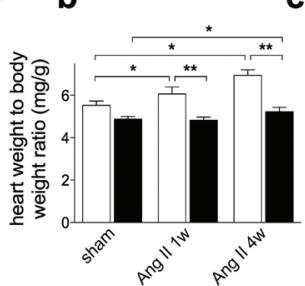

e
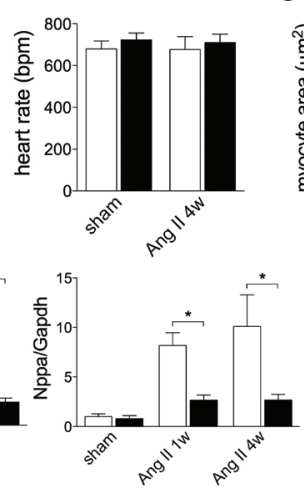

C

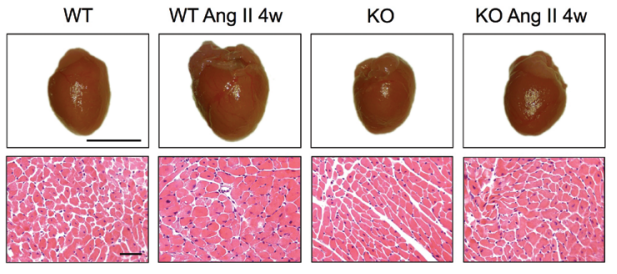

$\mathbf{f}$
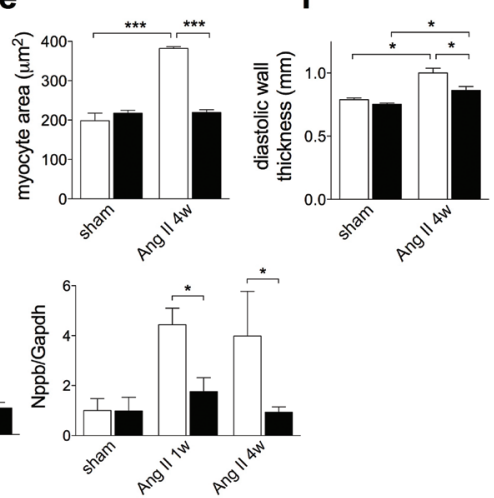

Figure 1: MiR-155 KO mice are protected from Ang II-induced cardiac hypertrophy and failure. (A) Fractional shortening as a measure of cardiac function shows no significant differences between miR155 WT and KO mice at baseline. WT mice develop systolic dysfunction after 4 weeks of Ang II, while KO mice are protected. (B) Heart weight to body weight ratios of miR-155 WT and KO mice are not different at baseline. WT mice but not KO mice develop cardiac hypertrophy following Ang II, already apparent at week 1 and pronounced after 4 weeks. (C) Representative images of whole hearts (top panels, scale bar:

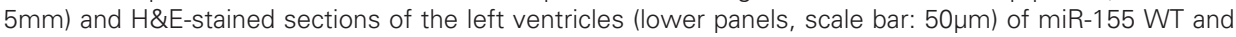
KO mice. (D) Tail cuff measurements of arterial blood pressure in WT and KO mice after 3 weeks of Ang II infusion reveal a comparable development of hypertension in both genotypes measured under similar heart rates. Quantifications of myocyte cross-sectional area (E), diastolic averaged wall thickness (F) and cardiac expression of the fetal hypertrophy marker genes Acta1, Nppa and Nppb (G) all show an increase in Ang II-treated miR-155 WT mice, but to a significantly lesser extent in KO mice. WT sham, $n=13$; WT Ang II 1W, $n=4$; WT Ang $\| 4 w, n=11 ; K O$ sham, $n=11 ; K O$ Ang $\| 1 w, n=5 ; K O$ Ang $\| 4 w, n=14$; all error bars represent s.e.m.; ${ }^{*} P<0.05,{ }^{*} P<0.01,{ }^{*}{ }^{*} P<0.005$.

adult WT mice followed by Ang II treatment for 4 weeks. We obtained a cardiac knockdown of $81 \pm 4 \%$ in antimiR-155-treated mice as compared to mice treated with control antimiR (Figure 4A). Similar to the miR-155 KO mice, antimiR-155-treated mice were protected from hypertrophy and heart failure following Ang II. MiR-155 inhibition was accompanied by preserved systolic function (Figure 4B), decreased cardiac weight and wall thickness (Figure 4C-E and Supplementary Table 1), and decreased induction of Acta1, Nppa and Nppb (Figure 4F) compared to LNA-control Ang II. Again, interstitial fibrosis induced by Ang II did not significantly differ in both groups (Figure 4G and Supplementary Table 1).

Together, these data show that, in the absence of miR-155, cardiac myocytes do not respond to pressure overload-induced stress by pathological remodeling, and are protected from cardiac dysfunction in response to both pharmacological and physical pressure overload. Therefore, we conclude that miR-155 mediates cardiac function under pathological conditions. 


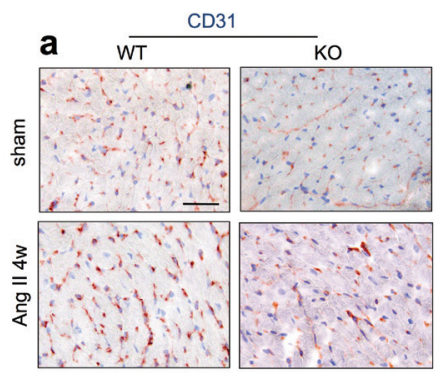

口 WT $\square$ miR-155 KO b

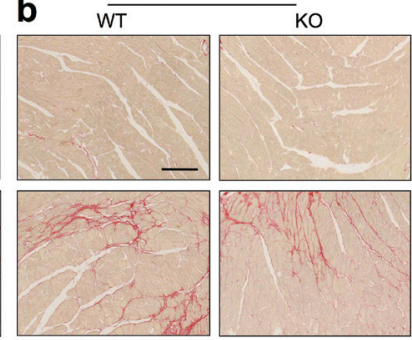

d

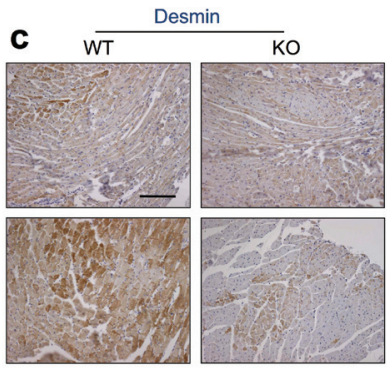

口WT $\square$ miR-155 KO
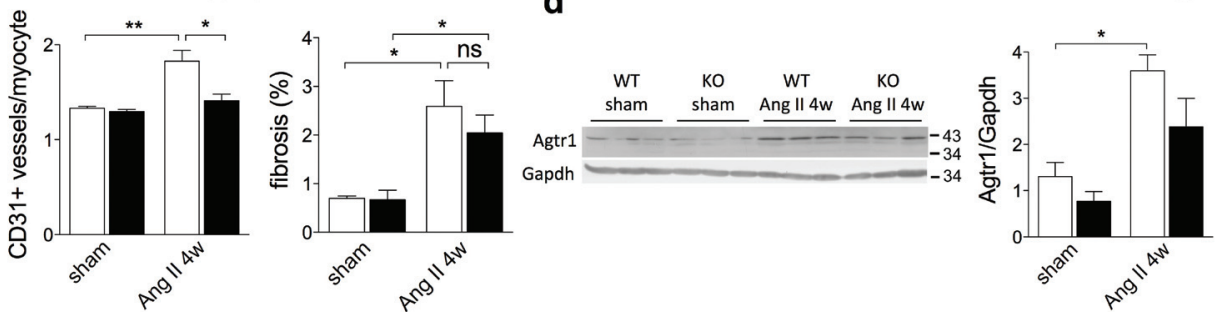

Figure 2: Protection from cardiac hypertrophy in the absence of miR-155 is independent of vascularization, fibrosis and Agtr1 expression. (A) The number of CD31-positive vessels per myocyte is similar between miR-155 WT and KO hearts at baseline, but increases only in WT hearts in response to Ang II. (B) Quantification of Sirius red staining shows comparable fibrosis in response to Ang Il-induced pressure overload in both genotypes (scale bar: $100 \mu \mathrm{m}$ ) while $(\mathrm{C})$ desmin staining as a marker of cardiac stress shows a marked increase in WT hearts but not KO hearts subjected to Ang II (scale bar: $100 \mu \mathrm{m})$. (D) Protein levels of the Agtr1 are not significantly different between the hearts of WT and KO mice both at baseline and in response to Ang II infusion. WT sham, $n=13$; WT Ang II 4w, $n=11$; KO sham, $n=11$; KO Ang II 4w, $n=14$; all error bars represent s.e.m.; ${ }^{*} P<0.05,{ }^{*} P<0.01$.

\section{MiR-155 controls cardiac monocyte/macrophage infiltration upon pressure overload}

To establish the cellular origin of miR-155 in the heart, we visualized cardiac miR-155 distribution by in situ hybridization. Almost no miR-155 signal was observed in cardiac myocytes and the interstitial compartment of sham hearts. However, following Ang IItreatment we observed marked miR-155 induction localized to infiltrating patches of interstitial leukocytes in miR-155 WT but not KO hearts (Figure 5A). Given its well-known immune role and its expression in cardiac inflammatory cells during pressure overload, we hypothesized that miR-155 may influence the function of leukocytes in the heart during stress. Immunostaining for inflammatory cell markers revealed that, following Ang II treatment, WT hearts experienced an influx of macrophages and - to a much lesser extent - T cells already after 1 week and lasting for at least 4 weeks (Figure 5B-E and Supplementary Figure 1A). However, absence of miR-155 largely abrogated this influx both in miR-155 KO and antimiR-155-treated mice (Figure 5B-E and Supplementary Figure 1A). Similarly, TAC triggered a significant recruitment of leukocytes to WT but not to KO hearts (Supplementary Figure 1B). Baseline numbers of interstitial leukocytes were not affected by absence of miR-155. These observations point towards a role for miR-155 to drive macrophage mobilization and infiltration upon pressure overload. 
In line with these data, the secretome of LPS-activated miR-155 KO macrophages showed a broadly reduced activation status as compared to WT macrophages. Levels of proteins indicative of macrophage activation or important for tissue infiltration, including the macrophage receptor mac1, the adhesion-mediating integrins LFA1 and VLA4a, and the inflammatory cytokines TNFa, IL1B and IL12, were significantly lower in the miR-155 KO macrophage secretome, suggesting compromised macrophage function upon activation in the absence of miR-155 (Figure 6A).

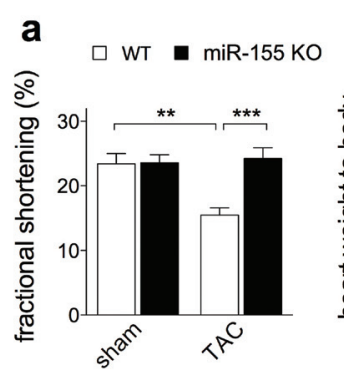

b

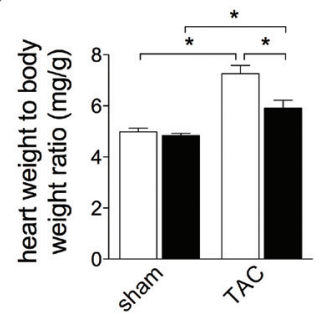

C

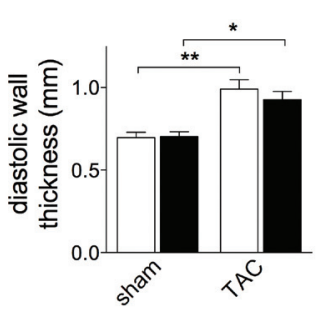

Sirius red d

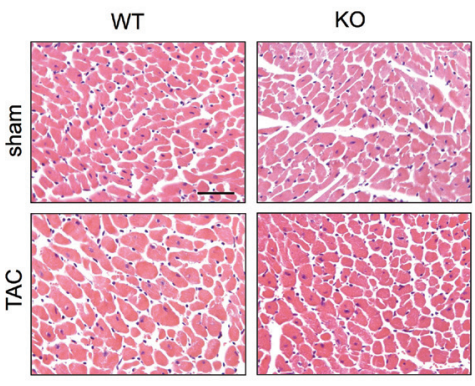

$\square$ WT $\square$ miR-155 KO
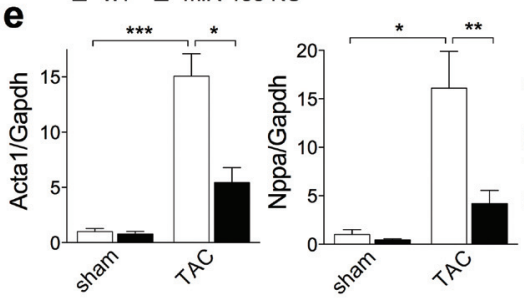

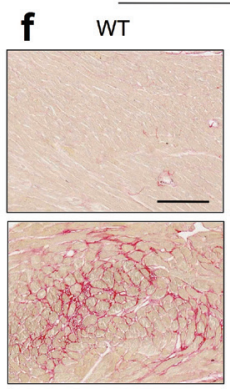

KO

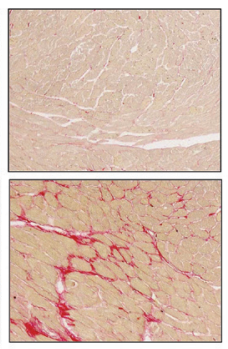

f
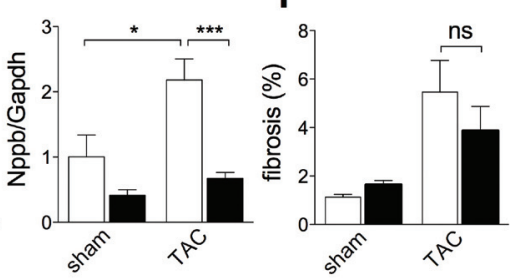

Figure 3: MiR-155 KO mice are protected from TAC-induced cardiac hypertrophy and failure. (A) Echocardiographic analysis shows that miR-155 KO but not WT mice are protected from systolic dysfunction following transverse aortic constriction (TAC) for 4 weeks. (B) Both WT and KO mice develop cardiac hypertrophy following TAC, but this response is significantly blunted in KO mice. (C) Both WT and KO mice significantly increase averaged diastolic wall thicknesses following TAC with a trend to lower wall thickness in KO mice. (D) Representative images of H\&E-stained sections of the left ventricles of miR-155 WT and KO

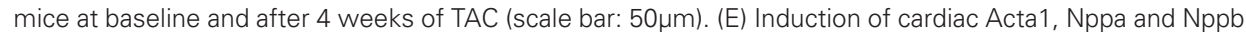
expression is significantly blunted in KO mice following TAC. (F) Quantification of Sirius red staining shows comparable fibrosis in response to TAC-induced pressure overload in both genotypes (scale bar: $100 \mu \mathrm{m}$ ). WT sham, $n=5 ;$ WT TAC, $n=6$; KO sham, $n=11$; KO TAC, $n=11$; all error bars represent s.e.m.; * $P<0.05$, ${ }^{* *} P<0.01,{ }^{* *} P<0.005$. 


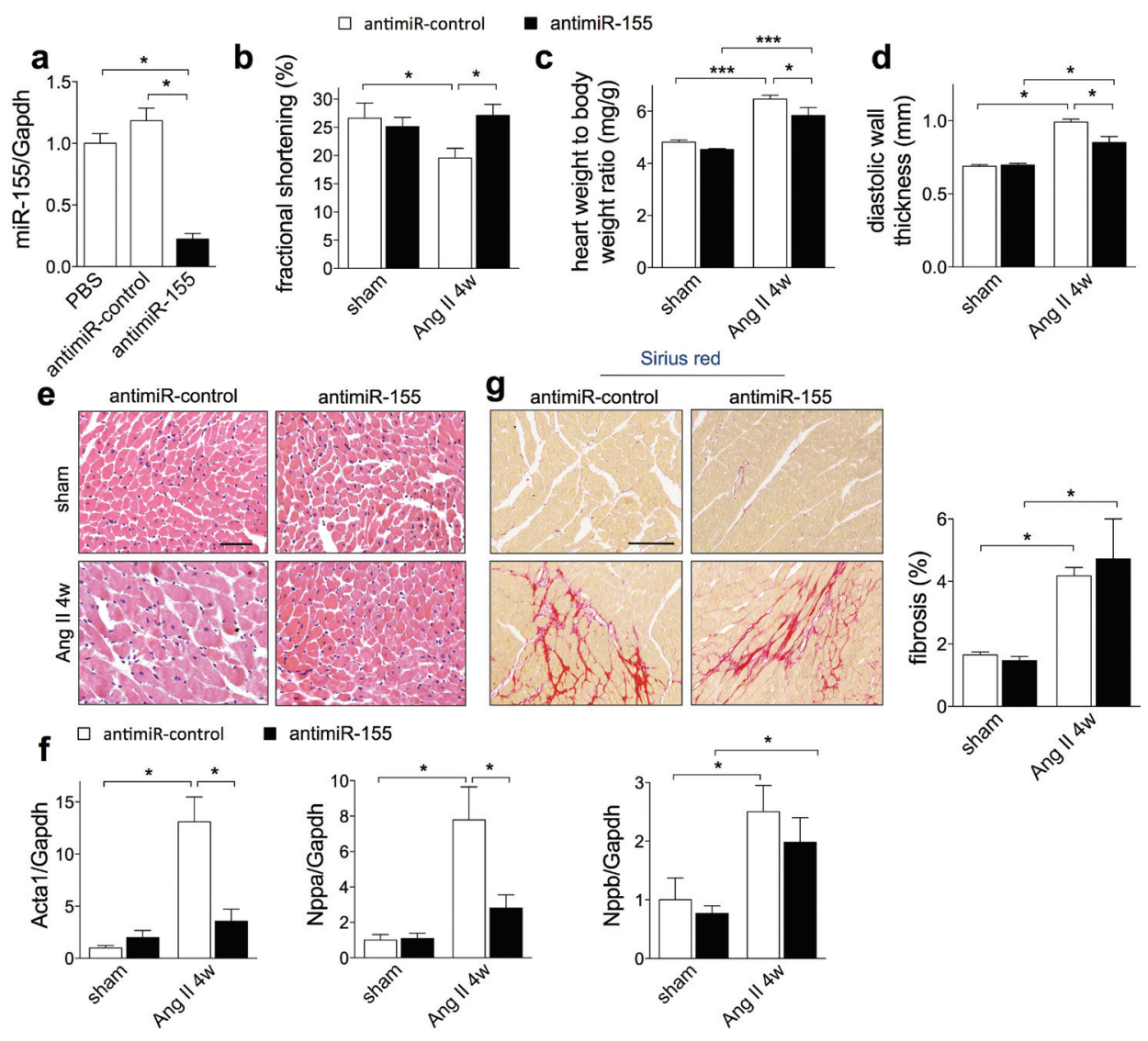

Figure 4: AntimiR-155 treatment protects against Ang II-induced cardiac hypertrophy and failure. (A) qRT-PCR analysis of miR-155 levels in mouse hearts 4 weeks after treatment with antagomiRs shows significant knockdown by antagomiRs against miR-155. AntimiR-mediated miR-155 inhibition protects WT mice from Ang II-induced systolic dysfunction (B) and cardiac hypertrophy (C, D). (E) Representative images of H\&E-stained sections of the left ventricles of miR-155 WT and KO mice at baseline and after 4 weeks of Ang II infusion (scale bar: $50 \mu \mathrm{m}$ ). (F) Induction of cardiac hypertrophic marker genes Acta1 and Nppa is significantly reduced in antimiR-155 treated mice following Ang II, while Nppb induction does not differ significantly. (G) Quantification of Sirius red staining shows comparable fibrosis in response to Ang II-induced pressure overload after control and anti-miR-155 antagomirs (scale bar: $100 \mu \mathrm{m})$. WT scrambled sham, n=6; WT scrambled Ang II, n=8; KO scrambled sham, n=6; KO scrambled Ang II, n=6; all error bars represent s.e.m., ${ }^{*} P<0.05,{ }^{*} P<0.01,{ }^{*}{ }^{*} P<0.005$.

Finally, flow cytometry of circulating leukocytes revealed that 1 week Ang II failed to mobilize CD11b+/Ly6G- monocytes in the absence of miR-155 (Figure 6B), while CD11b+/ Ly6G+ granulocytes were equally mobilized by Ang II in WT and KO (Supplementary Table 2). Again, baseline numbers of circulating monocytes and granulocytes were similar in WT and $\mathrm{KO}$ mice. In agreement with previous finding $\mathrm{s}^{24}, \mathrm{KO}$ mice were found to have a reduced number of regulatory $T$ cells. In conclusion, these data show that monocyte mobilization, cardiac macrophage infiltration and macrophage activity are hampered in response to pressure overload in miR-155-deficient mice. Combined with the protection of miR-155 
$\mathrm{KO}$ mice against pressure overload-induced HF, these data suggest that macrophage presence and activity are important pathogenic factors during the cardiac hypertrophic response and the progression to HF.

\section{Macrophages influence hypertrophic growth of cardiac myocytes via miR-155}

To pursue the hypothesis that macrophage miR-155 promotes cardiac hypertrophy, we undertook two distinct approaches. First, we investigated the myocyte hypertrophic response both in vivo and in vitro with miR-155 specifically absent in inflammatory cells. In vivo, we adoptively transferred WT bone marrow into miR-155 KO mice (WT to KO) and vice versa (KO to WT) and infused Ang II for 4 weeks. The presence of WT macrophages in miR-155 KO mice increased the cardiac hypertrophic response (Figure 7A).
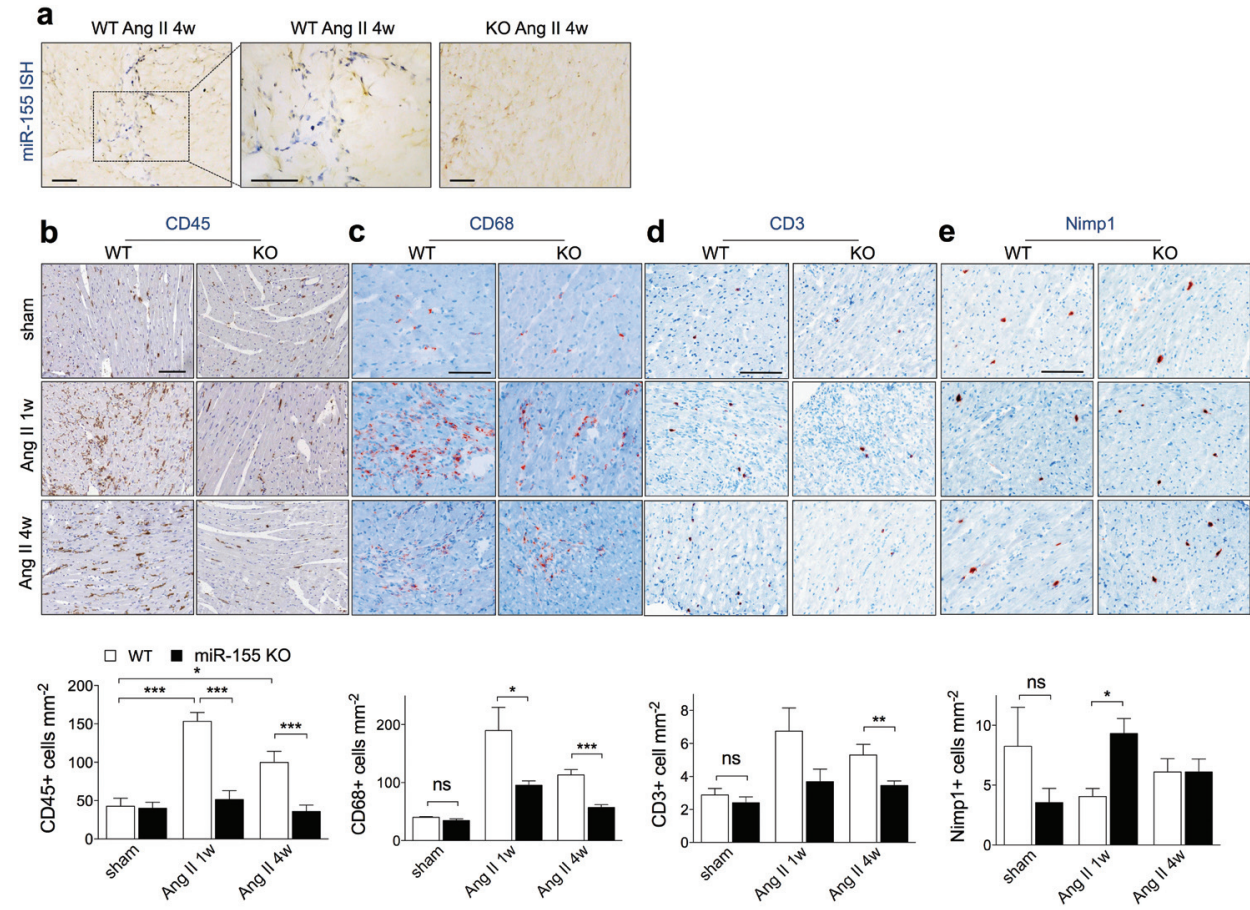

Figure 5: MiR-155 is required for pressure overload-induced influx of macrophages in the heart. (A) In situ hybridization of miR-155 in cardiac sections. While miR-155 expression is hardly detected in myocytes and interstitium of sham hearts, miR-155 signal is readily detected in patchy infiltrates of inflammatory cells after Ang II treatment. No signal was observed in KO hearts (scale bars: $50 \mu \mathrm{m})$. Quantification of immunoreactive cells using antibodies against CD45 (all leukocytes; B), CD68 (macrophages; C), CD3 (T-cells; D) or NIMP-1 (neutrophils; E). Macrophages have infiltrated the heart after 1 and 4 weeks of Ang II-induced pressure overload in WT mice, but this is significantly depressed in hearts of miR-155 KO mice. In addition, infiltration of T-cells - though substantially lower in number as compared to macrophages - is significantly reduced in miR-155 KO versus WT hearts, while numbers of NIMP-1-positive neutrophils are not different at baseline and 4 weeks of Ang $\mathrm{II}$ and show higher numbers of infiltrating cells in KO hearts after 1 week. All error bars represent s.e.m.; $n=4$ /group; ${ }^{*} P<0.05,{ }^{*} P<0.01,{ }^{*}{ }^{*} P<0.005$. Scale bars: $100 \mu \mathrm{m}$. 
Also the expression of cardiac stress markers Nppa and Nppb - but not Acta1 - was rescued by the replacement of KO macrophages by WT (Figure 7B). Vice versa, the removal of macrophage miR-155 from WT mice compromised hypertrophic growth following Ang II (Figure 7A), and cardiac stress markers Nppa, Nppb and Acta1 decreased towards full KO levels (Figure 7B). Although we did not accomplish a full rescue of hypertrophy, which is possibly due to a fraction of host macrophages remaining after irradiation ${ }^{25}$, these findings suggest that miR-155 controls pro-hypertrophic signaling by macrophages. To independently confirm this mechanism in vitro, we grew neonatal rat cardiac myocytes in conditioned culture media of LPS-activated WT and KO bone marrowderived macrophages. Interestingly, cardiac myocytes cultured in miR-155 KO macrophage media displayed a limited hypertrophic response to endothelin-1 as compared to WT macrophage media-cultured cardiac myocytes, demonstrated by Acta1 and Nppa gene expression and myocyte size (Figure 7C).

Second, though the expression of miR-155 by cardiac myocytes is low (Figure 5A), we ruled out a possible role of cardiac myocyte miR-155 to drive hypertrophy both in vivo and in vitro.

a
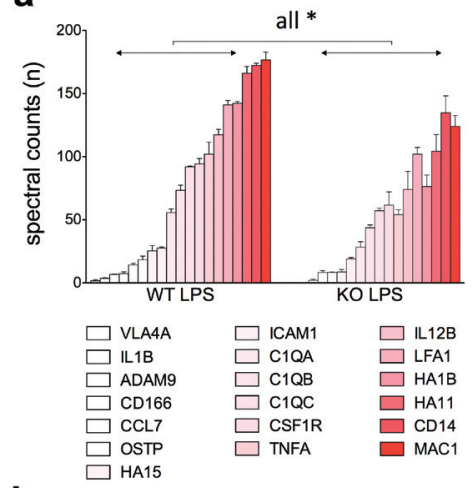

b

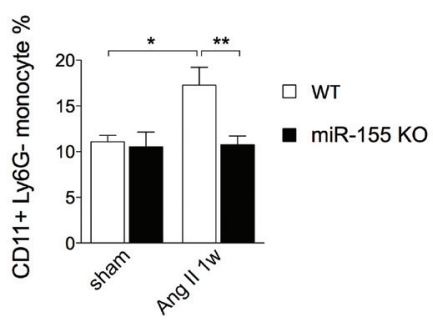

C
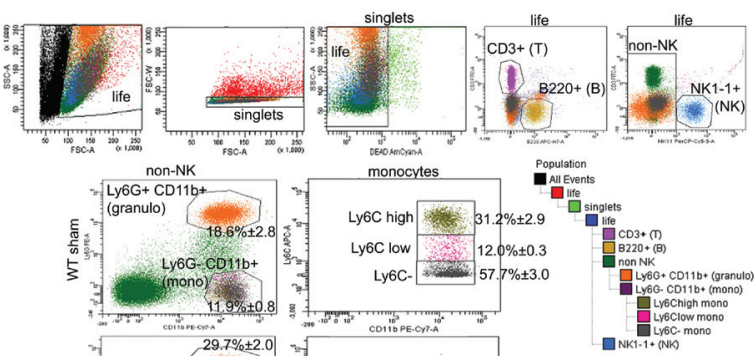
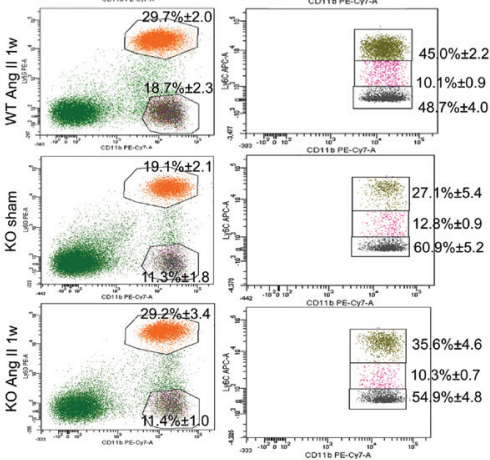

Figure 6: MiR-155 controls macrophage mobilization and activity. (A) Secreted abundance of selected macrophage proteins in WT LPS BMM media is significantly higher than in KO LPS BMM media $(n=3 /$ group). (B, C) Flow cytometry of circulating blood cells after 1 week of Ang II treatment shows impaired mobilization of CD11b+/Ly6G-/NK1.1-monocytes in KO animals, while CD11b+/Ly6G+/NK1.1- granulocyte mobilization following Ang II is unaffected by miR-155 ( $n=6 /$ group). Panel $C$ shows the gating strategy and representative flow cytometry pictures with averages and standard errors of immune cell subpopulations. All error bars represent s.e.m.; ${ }^{*} P<0.05,{ }^{*} P<0.01,{ }^{*}{ }^{*} P<0.005$. 

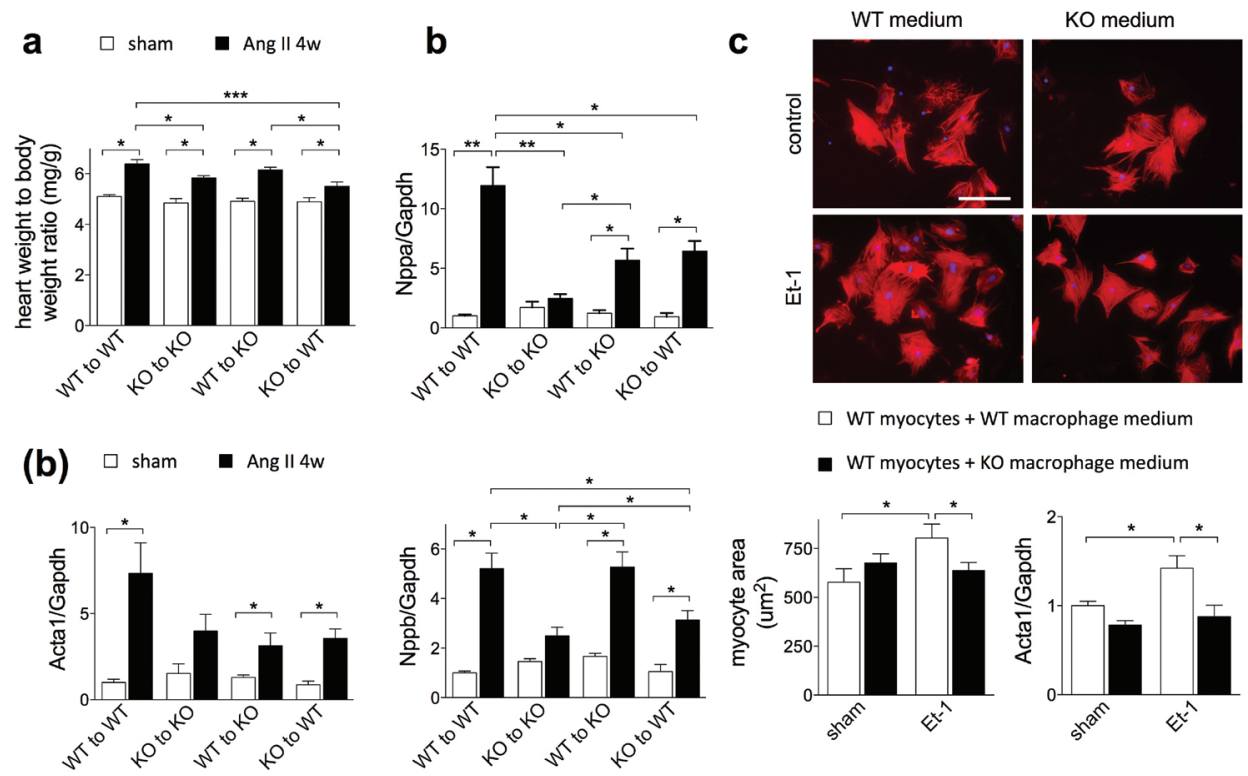

Figure 7: Macrophages influence hypertrophic growth of cardiac myocytes via miR-155. Heart weight to body weight ratios (A) and cardiac Acta1, Nppa and Nppb (B) expression of Ang II- or sham-treated mice following adoptive bone marrow transfer between miR-155 WT and KO mice show partial rescue of the hypertrophic response of KO mice reconstituted with WT bone marrow as well as blunting of the hypertrophic response in WT mice reconstituted with KO bone marrow. (C) Phalloidin staining (red) of actin filaments in cultured neonatal rat cardiac myocytes (scale bar: $50 \mu \mathrm{m}$ ). The hypertrophic growth and Acta1 gene expression response of myocytes to Et-1 is significantly blunted by addition of culture media from miR-155 KO bone marrow-derived macrophages. All error bars represent s.e.m.; WT $>W T$ sham, $n=7$; WT $>$ WT Angll, $n=13 ; \mathrm{KO}>\mathrm{KO}$ sham, $\mathrm{n}=7$; KO $>\mathrm{KO}$ Angll, $\mathrm{n}=7$; WT $>\mathrm{KO}$ sham, $\mathrm{n}=7$; WT $>\mathrm{KO}$ Angll, $\mathrm{n}=11$; $\mathrm{KO}>\mathrm{WT}$ sham, $\mathrm{n}=5$; KO $>\mathrm{WT}$ Angll, $\mathrm{n}=12$; ${ }^{*} \mathrm{P}<0.05,{ }^{*} \mathrm{P}<0.01,{ }^{*}{ }^{*} \mathrm{P}<0.005$. All quantitative in vitro data were generated from a minimum of three replicates.

In vivo, we introduced hsa-pre-miR-155 in WT mice specifically into cardiac myocytes using an adeno-associated virus serotype 9 (AAV9)-vector and observed no difference in cardiac hypertrophy neither at baseline nor following Ang II (Figure 8A-C). Vice versa, inhibition of cardiac myocyte miR-155 function using AAV9-introduced miR-155 sponges demonstrated again similar cardiac mass and hypertrophic marker responses following Ang II infusion in control sponge and miR-155 sponge treated WT mice (Figure 8D-F). In addition, we knocked down miR-155 in vitro in cardiac myocytes and induced hypertrophy with endothelin-1, after which we could not observe differences in induction of hypertrophic markers (Figure $8 \mathrm{G}-\mathrm{H})$. Together, these data show that macrophage- but not myocyte-expression of miR155 regulates hypertrophic cardiomyocyte growth in a paracrine fashion.

\section{The Socs1/II6/Stat3 signaling axis links macrophage miR-155 to cardiac myocyte hypertrophic growth}

We next addressed how miR-155 expression in macrophages influences macrophage paracrine activity. Direct miR-155 targets that potentially modulate macrophage activity and could explain the observed phenotype include Src homology 2-containing inositol 
phosphatase-1 (Ship-1) ${ }^{26}$ and Socs ${ }^{127}$. We found that in the absence of miR-155, Socs 1 protein expression is indeed elevated in untreated as well as LPS-activated macrophages as compared to WT macrophages, while Ship1 was found mildly derepressed only in unstimulated macrophages (Figure 9A-B). Therefore, we focused on Socs1, which inhibits macrophage inflammatory responses by targeting the Jak/Stat axis ${ }^{28}{ }^{29}$. In agreement, we found that the elevated Socs1 levels in LPS-treated miR-155-deficient macrophages were associated with diminished Stat3 phosphorylation at baseline and at 6 and 24 hours following LPS stimulation (Figure 9A and C), consistent with diminished pro-inflammatory

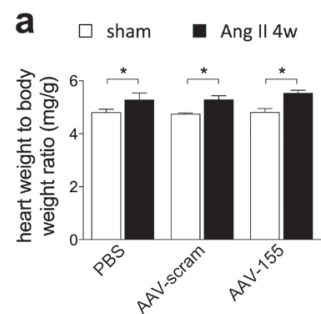

d $\square$ AAV-sponge control b

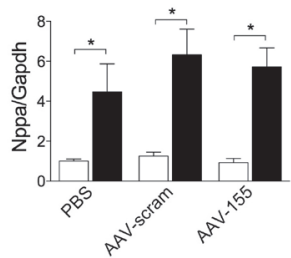

e
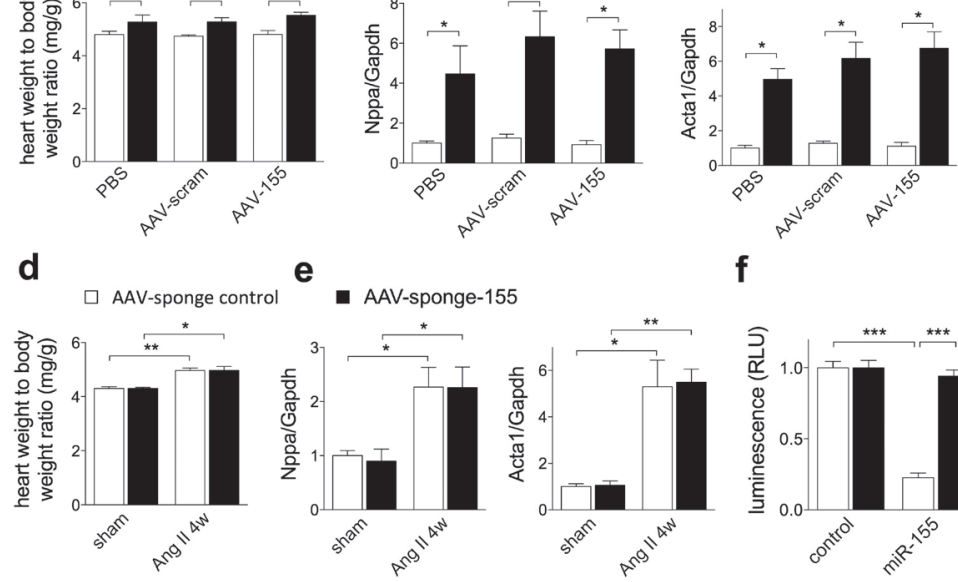

C
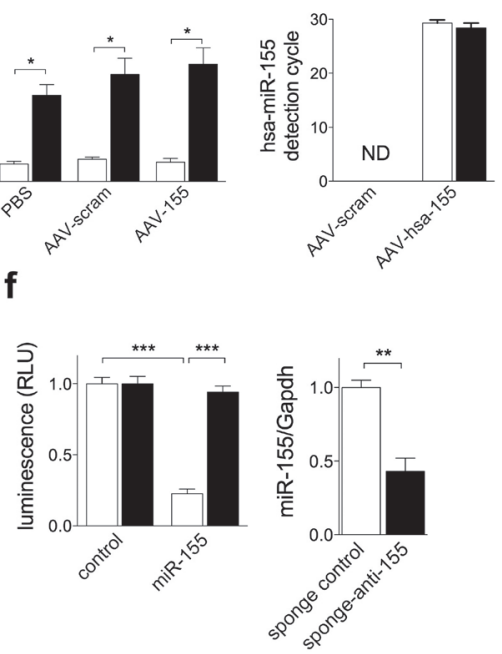

g $\square$ WT $\square \operatorname{miR}-155 \mathrm{KD} \quad \mathbf{h}$
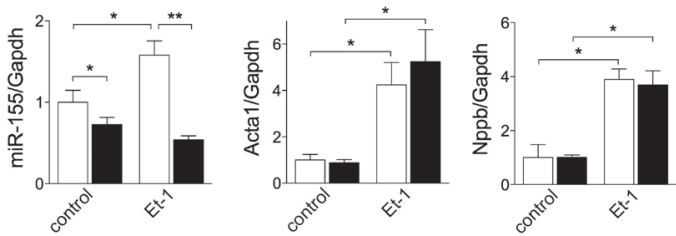

Figure 8: Myocyte-specific manipulation of miR-155 has no influence on the development of cardiac hypertrophy. In vivo cardiac myocyte miR-155 overexpression using AAV9-hsa-miR-155 leads to similar induction of cardiac hypertrophy (A) and of the fetal genes Nppa and Acta1 (B), as compared to both PBS and AAV9-scrambled treatment. (C) Human miR-155 was detected only in AAV9-hsa-miR-treated mouse groups. Note that human and mouse miR-155 differ by one nucleotide outside the seed region, enabling human-specific miR-155 detection. Vice versa, in vivo cardiac myocyte miR-155 inhibition using AAV9sponge-miR-155 also induces similar levels of cardiac hypertrophy (D) and of hypertrophic gene expression (E). (F) Luciferase validation of sponge efficiency shows a knockdown of the luciferase target to $23 \% \pm 3$ (left panel). Co-expression of sponge-miR-155 rescued this knockdown (>90\%) whereas expression of a sponge containing 4 mutated binding sites had no influence. In vivo, AAV9-sponge-miR-155 reduced miR155 expression by $57 \% \pm 7$ (right panel). Knockdown of miR-155 in neonatal rat cardiac myocytes with antagomiRs (G) does not influence induction of the fetal genes Acta1 and Nppb (H). All error bars represent s.e.m.; PBS sham, $n=8$; PBS Ang II, $n=8$; AAV-scram sham, $n=8$; AAV-scram Ang II, $n=12$; AAV-155 sham, $n=6 ; A A V-155$ Ang II, $n=8 ;{ }^{*} P<0.05,{ }^{*} P<0.01 ; N D$, not detected. All quantitative in vitro data were generated from a minimum of three replicates. 


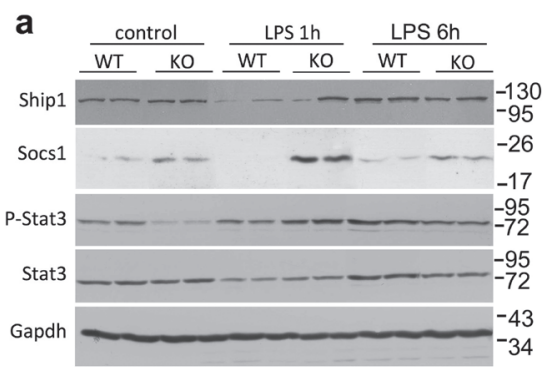

b
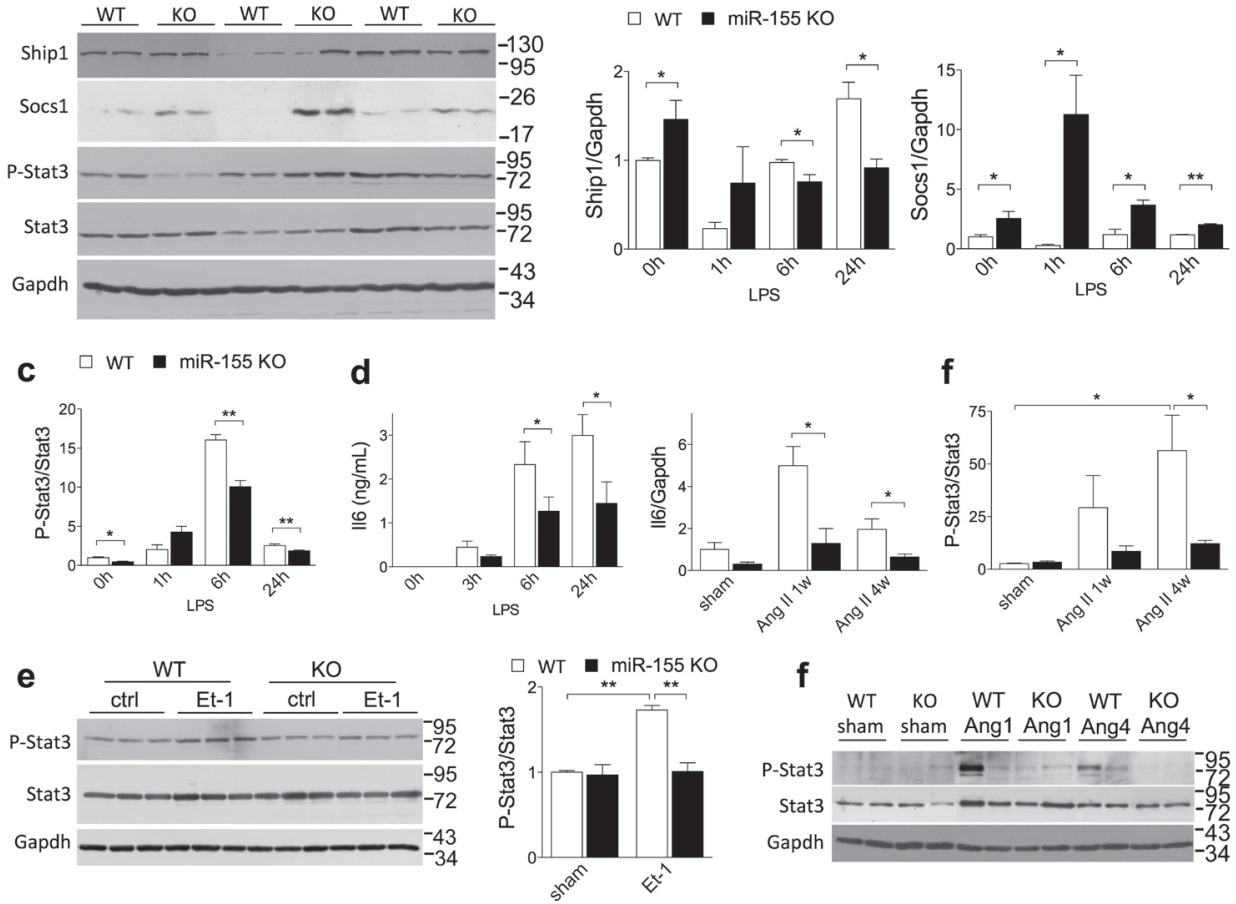

Figure 9: MiR-155 controls inflammatory signaling through its target Socs1. (A-C) Representative Western blot images and quantification of Ship1, Socs1 and Stat3 phosphorylation (P-Stat3) in bone-marrow derived macrophages from miR-155 WT or KO mice stimulated with LPS. MiR-155 KO macrophages show higher Socs1 levels and lower Stat3 activation (P-Stat3/Stat3 levels) in response to LPS versus WT macrophages. (D) Levels of $I 16$ are higher in WT versus KO macrophages in response to LPS (ELISA), and in WT versus KO mouse hearts following 1 and 4 weeks of Ang II infusion (mRNA). Levels of Tnfa are not affected by absence of miR-155 in macrophages, and are even temporarily and slightly elevated shortly after LPS stimulation, while cardiac mRNA levels are temporarily and slightly down-regulated after Ang II infusion. (E) Adding culture media from KO macrophages to neonatal cardiac myocytes inhibits their Stat3 phosphorylation in response to Et-1 stimulation. (F) Stat-3 phosphorylation is significantly lower in KO versus WT mouse hearts after 1 and 4 weeks of Ang II infusion (WT sham, $n=5$; WT Angll 1W, $n=4$; WT Angll 4W, $\mathrm{n}=6$; $\mathrm{KO}$ sham, $\mathrm{n}=6$; KO Angll $1 \mathrm{w}, \mathrm{n}=4$; $\mathrm{KO}$ Angll $4 \mathrm{w}, \mathrm{n}=4$ ). All error bars represent s.e.m., ${ }^{*} \mathrm{P}<0.05,{ }^{*} \mathrm{P}$ $<0.01,{ }^{* *} P<0.005$. Western blot images are representative. All quantitative in vitro data were generated from a minimum of three replicates.

responses in the absence of miR-155. Activated miR-155 deficient macrophages secrete less pro-inflammatory and pro-hypertrophic cytokines, including interleukin-6 (II-6) (Figure 9D) and tumor necrosis factor-alpha (Tnfa) (Figure 6A). Interestingly, both are involved in hypertrophic and inflammatory signaling; II-6 via Stat3 and Tnfa by activation of nuclear factor-kappaB (Nf-kB) ${ }^{30-32}$.

Hypothesizing that II6-dependent activation of Stat3 in myocytes could mediate the prohypertrophic effects of activated macrophages, we studied Stat3 activation in cardiac myocytes subjected to conditioned macrophage media. We found decreased P-Stat3 levels in the cardiac myocytes grown in miR-155-deficient macrophage media (Figure 9E). Similar results were obtained in vivo, where miR-155-deficient hearts showed dramatically reduced 
Stat3 activities upon pressure overload (Figure 9F). We found no evidence for involvement of Nf-kB signaling in limiting hypertrophy in the absence of miR-155 (not shown). Together, these data support a central role for Stat3 signaling in miR-155-driven cardiac hypertrophy and failure.

To confirm that macrophage-expressed miR-155 mediates cardiac hypertrophy via Socs1, we prevented the de-repression of Socs 1 in miR-155 KO macrophages with siRNAs (Figure 10A).

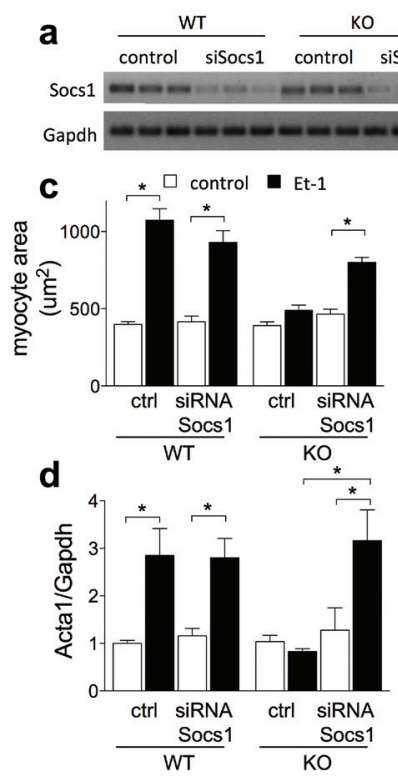

b
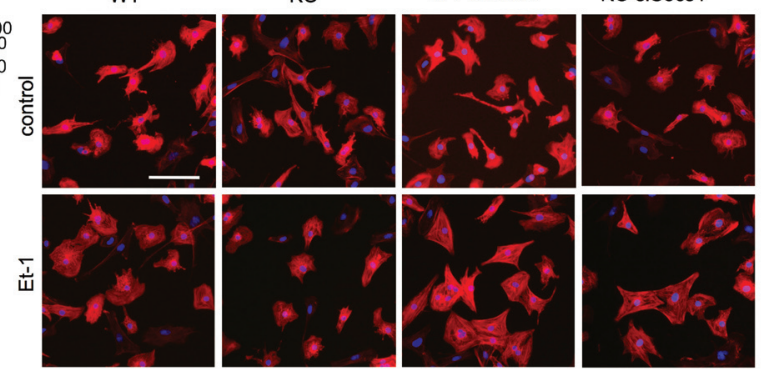

$\square$ control $\square$ Et-1
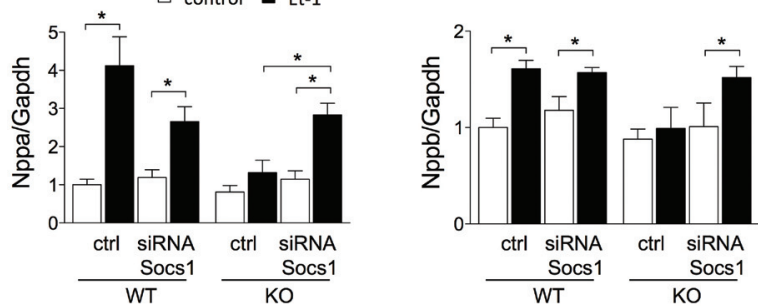

\section{e}

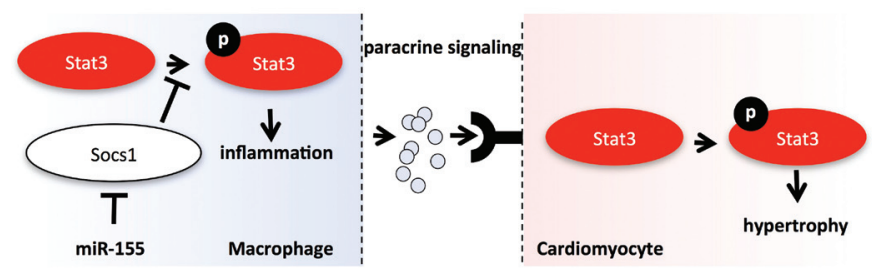

Figure 10: Socs1 knockdown in macrophages rescues paracrine hypertrophic stimulation to myocytes. (A) Knockdown of Socs1 transcripts in bone marrow-derived macrophages from miR-155 WT and KO mice. Socs 1 is repressed by miR-155 on the translational level; therefore transcript levels are similar between WT and KO cells. (B) Phalloidin staining (red) of actin filaments in cultured neonatal rat cardiac myocytes stimulated with Et-1 and grown in culture media from WT or KO bone marrow-derived macrophages that were treated with siRNAs against Socs1 or control (scale bar: $50 \mu \mathrm{m})$. Quantification of cross-sectional myocyte areas (C) and expression of hypertrophic markers Acta1, Nppa and Nppb (D) show that the blunting of hypertrophy by media from KO macrophages is rescued by Socs1 knockdown in macrophages. (E) Schematic representation of the cardiac miR-155 signaling pathway. MiR-155 inhibits Socs1, enabling the pressure overload-induced increase in 116 levels as well as directly relieving Socs1-mediated repression of macrophage Stat3 activity. II6 signals to macrophages and cardiac myocytes to induce Stat3 activity, leading to inflammatory activation and cardiac myocyte hypertrophy. All error bars represent s.e.m., ${ }^{*} P<0.05,{ }^{*} P$ $<0.01,{ }^{* *} P<0.005$. All quantitative in vitro data were generated from a minimum of three replicates. 
Socs1 knockdown in macrophages largely rescued the limited hypertrophic response of myocytes grown in miR-155 KO macrophage-conditioned media both at the level of myocyte growth and gene expression (Figure 10B-E). Therefore, we conclude that the paracrine effects of miR-155 on myocyte hypertrophy are mediated at least in part by its direct target Socs1.

\section{Discussion}

In this study, we identify a role for the macrophage-derived miR-155 in cardiac hypertrophy and failure in response to pressure overload. Gene inactivation or pharmacological inhibition of miR-155 significantly reduced the hypertrophic growth and prevented systolic dysfunction in pressure overloaded hearts. A role for miR-155 specifically derived from macrophages was indicated by the fact that transplantation of miR-155 KO bone marrow into irradiated WT mice blunted the hypertrophic response, whereas reconstitution of WT macrophages in miR-155 KO mice largely rescued the hypertrophic response. In line with these findings, miR-155 in situ hybridization co-localized with the areas of interstitial inflammation in the pressure overloaded WT hearts. Moreover, we specifically exclude a role for cardiomyocytederived miR-155 in cardiac hypertrophy, since both AAV9-mediated cardiomyocytic overexpression and knockdown of miR-155 had no effect on the in vivo cardiac hypertrophic response. As additional proof in vitro, macrophage medium derived from miR-155 KO animals blunted the hypertrophic growth of cardiomyocytes, while medium from WT macrophages did allow hypertrophy.

The mechanism responsible for cardioprotection of miR-155 KO mice was partly mediated by de-repression of the translational miR-155 target Socs1 in inflammatory cells. In vitro, Socs1 knockdown in miR-155 KO macrophages rescued the lack of cardiomyocyte hypertrophy. Socs1 increase in the absence of miR-155 reduces the release of prohypertrophic and -inflammatory cytokines such as 116 and Tnfa, thereby protecting cardiac cells from chronic cytokine-mediated activation of pro-hypertrophic signaling pathways. As such, miR-155 links hypertension-induced interstitial cardiac inflammation to myocyte hypertrophy and function (a diagram of the proposed signaling pathway is provided in Figure 10E). Our finding that leukocytes may regulate cardiac hypertrophy is in line with previous studies revealing a role for leukocyte-derived galectin-3 and PI3 kinase in cardiac hypertrophic remodeling ${ }^{5,33,34}$. Furthermore, reduction of cardiac monocyte infiltration by administration of immune-inhibitory regulatory $T$ cells prevented cardiac hypertrophy and damage during Ang II-mediated pressure overload ${ }^{35}$, and II6-null mice are protected from cardiac hypertrophy irrespective of blood pressure changes ${ }^{36}$. These studies and the current work imply that inflammatory signaling may be necessary for hypertrophic remodeling during pressure overload. Importantly, the cardiac influx of inflammatory cells upon pressure overload precedes signs of hypertrophy and heart failure (Figure 5B), suggesting a direct contribution to cardiac pathophysiology.

Cardioprotection of miR-155 KO mice was associated with reduced Stat3 activation and decreased II6 levels in cardiac myocytes as well as macrophages, in line with the de- 
repression of Socs1. On the one hand, Socs1 is an endogenous inhibitor of Stat3 activation, and on the other hand it is a potent inhibitor of cytokine production and release ${ }^{30}$. In turn, cytokines - including 116 - induce Stat3 signaling, that has both pro-hypertrophic and proinflammatory effects ${ }^{31,32}$. We propose that increased Socs 1 levels and resulting decreased Stat3 signaling reduce the production of cytokines in miR-155-deficient cardiac macrophages during pressure overload. This altered milieu of secreted factors blunts the hypertrophic response of miR-155-deficient myocytes, as evidenced by decreased Stat3 activation and decreased production of hypertrophic markers. The observed chronically reduced Stat3 activation during pressure overload in the absence of miR-155 was associated with improved cardiac function. Whereas rapid Stat3 activation in acute heart failure is cardioprotective ${ }^{37-39}$, chronic activation of this signaling pathway during pressure overload ${ }^{40}$ is detrimental and causes increased cardiac inflammation and failure ${ }^{37}$. This is reminiscent of other inflammatory pathways such as Toll-like receptor, Nuclear factor of activated T cells (NFAT) and NF-kB signaling, whose acute expression during cardiac disease is protective, while sustained expression is detrimenta| ${ }^{41-43}$. Therefore, chronic pressure overload may cause a prolonged, unbalanced Stat3 activation leading to adverse effects. In line, sustained activation of Stat3 following chronic Ang II infusion was associated with heart failure in our WT mice, and reduced Stat3 signaling in the absence of miR-155 may therefore in part contribute to the cardiac protection observed. Our data emphasize that cardiac function - like many other physiological processes - depends on balanced signaling activities.

In the present study, three essential components of the cardiac inflammatory effects of Ang II are affected by miR-155 deficiency: (1) Ang II-induced mobilization of leukocytes to the circulation; (2) leukocyte infiltration into the heart; and (3) production of pro-inflammatory and pro-hypertrophic factors by activated interstitial cardiac leukocytes. This study is the first to dissect the pro-inflammatory effects of Ang II during pressure overload. Our results indicate that miR-155 is crucial for leukocyte mobilization, tissue infiltration and activation upon pressure overload. Of note, while absence of miR-155 blunted pressure overloadinduced cardiac hypertrophy and inflammation, the cardiac fibrotic response remained intact, indicating that pressure overload-induced fibrotic remodeling was independent of miR-155 function in leukocytes. Damilano et al. show that leukocyte PI3K $y$ does influence cardiac fibrotic responses to TAC ${ }^{34}$. However, in agreement with our findings, these authors show that cardiac function is independent of fibrotic remodeling.

In conclusion, inflammatory signaling through miR-155 modulates cardiac hypertrophy and failure during pressure overload. Whereas pharmacological inhibition of miR-155 prevented cardiac hypertrophy and failure, its over-expression or knockdown in cardiomyocytes did not affect the hypertrophic response. Our data stress the importance of cardio-inflammatory crosstalk and identify miR-155 as a potential therapeutic target in heart failure.

\section{Acknowledgements}

We thank Laura de Rijck, Georg Summer, Dr Xiaoke Yin, Dr Hamid El Azzouzi, Nicole Bitsch, Paul Veulemans, Sylvia Jochems, Mathijs van de Vrie, Dr Jan D'Hooge, Dr Jack P Cleutjens, Ermira Samara, Janka Matrai, Dr Giulia Ruozi, Gregorio Fazzi and Linda Beckers for technical assistance and expertise; and Marinee Chuah and Thierry VandenDriessche for control AAV9 vectors. 


\section{References}

1. Jessup M, Brozena S. Heart failure. N Engl J Med. 2003;348:2007-2018

2. Heymans S, Hirsch E, Anker SD, Aukrust P, Balligand JL, Cohen-Tervaert JW, Drexler H, Filippatos G, Felix SB, Gullestad L, Hilfiker-Kleiner D, Janssens S, Latini R, Neubauer G, Paulus WJ, Pieske B, Ponikowski P, Schroen B, Schultheiss HP, Tschope C, Van Bilsen M, Zannad F, McMurray J, Shah AM. Inflammation as a therapeutic target in heart failure? A scientific statement from the translational research committee of the heart failure association of the european society of cardiology. Eur J Heart Fail. 2009;11:119-129

3. Mann DL. Inflammatory mediators and the failing heart: Past, present, and the foreseeable future. Circ Res. 2002;91:988-998

4. Marchant DJ, Boyd JH, Lin DC, Granville DJ, Garmaroudi FS, McManus BM. Inflammation in myocardial diseases. Circ Res. 2012;110:126-144

5. Kvakan H, Luft FC, Muller DN. Role of the immune system in hypertensive target organ damage. Trends Cardiovasc Med. 2009;19:242-246

6. Lee RC, Ambros V. An extensive class of small rnas in caenorhabditis elegans. Science. 2001;294:862-864

7. Care A, Catalucci D, Felicetti F, Bonci D, Addario A, Gallo P, Bang ML, Segnalini P, Gu Y, Dalton ND, Elia L, Latronico MV, Hoydal M, Autore C, Russo MA, Dorn GW, 2nd, Ellingsen O, Ruiz-Lozano P, Peterson KL, Croce CM, Peschle C, Condorelli G. Microrna-133 controls cardiac hypertrophy. Nat Med. 2007;13:613-618

8. Duisters RF, Tijsen AJ, Schroen B, Leenders JJ, Lentink V, van der Made I, Herias V, van Leeuwen RE, Schellings MW, Barenbrug P, Maessen JG, Heymans S, Pinto YM, Creemers EE. Mir-133 and mir-30 regulate connective tissue growth factor: Implications for a role of micrornas in myocardial matrix remodeling. Circ Res. 2009;104:170-178, 176p following 178

9. Thum T, Gross C, Fiedler J, Fischer T, Kissler S, Bussen M, Galuppo P, Just S, Rottbauer W, Frantz S, Castoldi M, Soutschek J, Koteliansky V, Rosenwald A, Basson MA, Licht JD, Pena JT, Rouhanifard SH, Muckenthaler MU, Tuschl T, Martin GR, Bauersachs J, Engelhardt S. Microrna-21 contributes to myocardial disease by stimulating map kinase signalling in fibroblasts. Nature. 2008;456:980-984

10. van Rooij E, Sutherland LB, Qi X, Richardson JA, Hill J, Olson EN. Control of stress-dependent cardiac growth and gene expression by a microrna. Science. 2007;316:575-579

11. Baltimore D, Boldin MP, O'Connell RM, Rao DS, Taganov KD. Micrornas: New regulators of immune cell development and function. Nat Immunol. 2008;9:839-845

12. Stanczyk J, Pedrioli DM, Brentano F, Sanchez-Pernaute O, Kolling C, Gay RE, Detmar M, Gay S, Kyburz D. Altered expression of microrna in synovial fibroblasts and synovial tissue in rheumatoid arthritis. Arthritis Rheum. 2008;58:1001-1009

13. Junker A, Krumbholz M, Eisele S, Mohan H, Augstein F, Bittner R, Lassmann H, Wekerle H, Hohlfeld R, Meinl E. Microrna profiling of multiple sclerosis lesions identifies modulators of the regulatory protein cd47. Brain. 2009;132:3342-3352

14. Xiao B, Liu Z, Li BS, Tang B, Li W, Guo G, Shi Y, Wang F, Wu Y, Tong WD, Guo H, Mao XH, Zou QM. Induction of microrna-155 during helicobacter pylori infection and its negative regulatory role in the inflammatory response. $\mathrm{J}$ Infect Dis. 2009;200:916-925

15. Sonkoly E, Janson P, Majuri ML, Savinko T, Fyhrquist N, Eidsmo L, Xu N, Meisgen F, Wei T, Bradley M, Stenvang J, Kauppinen S, Alenius H, Lauerma A, Homey B, Winqvist O, Stahle M, Pivarcsi A. Mir-155 is overexpressed in patients with atopic dermatitis and modulates t-cell proliferative responses by targeting cytotoxic t lymphocyte-associated antigen 4. J Allergy Clin Immunol. 2010;126:581-589 e581-520

16. O'Connell RM, Kahn D, Gibson WS, Round JL, Scholz RL, Chaudhuri AA, Kahn ME, Rao DS, Baltimore D. Microrna-155 promotes autoimmune inflammation by enhancing inflammatory t cell development. Immunity. 2010;33:607-619

17. Rodriguez A, Vigorito E, Clare S, Warren MV, Couttet P, Soond DR, van Dongen S, Grocock RJ, Das PP, Miska EA, Vetrie D, Okkenhaug K, Enright AJ, Dougan G, Turner M, Bradley A. Requirement of bic/microrna-155 for normal immune function. Science. 2007;316:608-611 
18. Thai TH, Calado DP, Casola S, Ansel KM, Xiao C, Xue Y, Murphy A, Frendewey D, Valenzuela D, Kutok JL, Schmidt-Supprian M, Rajewsky N, Yancopoulos G, Rao A, Rajewsky K. Regulation of the germinal center response by microrna-155. Science. 2007;316:604-608

19. O'Connell RM, Rao DS, Chaudhuri AA, Boldin MP, Taganov KD, Nicoll J, Paquette RL, Baltimore D. Sustained expression of microrna-155 in hematopoietic stem cells causes a myeloproliferative disorder. J Exp Med. 2008;205:585-594

20. Tili E, Croce CM, Michaille JJ. Mir-155: On the crosstalk between inflammation and cancer. Int Rev Immunol. 2009;28:264-284

21. Diguet N, Mallat Y, Ladouce R, Clodic G, Prola A, Tritsch E, Blanc J, Larcher JC, Delcayre C, Samuel JL, Friguet B, Bolbach G, Li Z, Mericskay M. Muscle creatine kinase deficiency triggers both actin depolymerization and desmin disorganization by advanced glycation end products in dilated cardiomyopathy. J Biol Chem. 2011;286:35007-35019

22. Schroen B, Leenders JJ, van Erk A, Bertrand AT, van Loon M, van Leeuwen RE, Kubben N, Duisters RF, Schellings MW, Janssen BJ, Debets JJ, Schwake M, Hoydal MA, Heymans S, Saftig P, Pinto YM. Lysosomal integral membrane protein 2 is a novel component of the cardiac intercalated disc and vital for load-induced cardiac myocyte hypertrophy. J Exp Med. 2007;204:1227-1235

23. Martin MM, Lee EJ, Buckenberger JA, Schmittgen TD, Elton TS. Microrna-155 regulates human angiotensin ii type 1 receptor expression in fibroblasts. J Biol Chem. 2006;281:18277-18284

24. Kohlhaas S, Garden OA, Scudamore C, Turner M, Okkenhaug K, Vigorito E. Cutting edge: The foxp3 target mir-155 contributes to the development of regulatory t cells. J Immunol. 2009;182:2578-2582

25. Kania G, Blyszczuk P, Valaperti A, Dieterle T, Leimenstoll B, Dirnhofer S, Zulewski H, Eriksson U. Prominin-1+/ cd133+ bone marrow-derived heart-resident cells suppress experimental autoimmune myocarditis. Cardiovasc Res. 2008;80:236-245

26. Kurowska-Stolarska M, Alivernini S, Ballantine LE, Asquith DL, Millar NL, Gilchrist DS, Reilly J, lerna M, Fraser AR, Stolarski B, McSharry C, Hueber AJ, Baxter D, Hunter J, Gay S, Liew FY, Mclnnes IB. Microrna-155 as a proinflammatory regulator in clinical and experimental arthritis. Proc Natl Acad Sci U S A. 2011;108:1119311198

27. Lu LF, Thai TH, Calado DP, Chaudhry A, Kubo M, Tanaka K, Loeb GB, Lee H, Yoshimura A, Rajewsky K, Rudensky AY. Foxp3-dependent microrna155 confers competitive fitness to regulatory $t$ cells by targeting socs1 protein. Immunity. 2009;30:80-91

28. Boengler K, Hilfiker-Kleiner D, Drexler H, Heusch G, Schulz R. The myocardial jak/stat pathway: From protection to failure. Pharmacol Ther. 2008;120:172-185

29. Dimitriou ID, Clemenza L, Scotter AJ, Chen G, Guerra FM, Rottapel R. Putting out the fire: Coordinated suppression of the innate and adaptive immune systems by socs1 and socs3 proteins. Immunol Rev. 2008;224:265-283

30. Heinrich PC, Behrmann I, Haan S, Hermanns HM, Muller-Newen G, Schaper F. Principles of interleukin (il)-6type cytokine signalling and its regulation. Biochem J. 2003;374:1-20

31. Hirota H, Yoshida K, Kishimoto T, Taga T. Continuous activation of gp130, a signal-transducing receptor component for interleukin 6-related cytokines, causes myocardial hypertrophy in mice. Proc Natl Acad Sci U S A. $1995 ; 92: 4862-4866$

32. Rebouissou S, Amessou M, Couchy G, Poussin K, Imbeaud S, Pilati C, Izard T, Balabaud C, Bioulac-Sage P, Zucman-Rossi J. Frequent in-frame somatic deletions activate gp130 in inflammatory hepatocellular tumours. Nature. 2009;457:200-204

33. Sharma UC, Pokharel S, van Brakel TJ, van Berlo JH, Cleutjens JP, Schroen B, Andre S, Crijns HJ, Gabius HJ, Maessen J, Pinto YM. Galectin-3 marks activated macrophages in failure-prone hypertrophied hearts and contributes to cardiac dysfunction. Circulation. 2004;110:3121-3128

34. Damilano F, Franco I, Perrino C, Schaefer K, Azzolino O, Carnevale D, Cifelli G, Carullo P, Ragona R, Ghigo A, Perino A, Lembo G, Hirsch E. Distinct effects of leukocyte and cardiac phosphoinositide 3-kinase gamma activity in pressure overload-induced cardiac failure. Circulation. 2011;123:391-399 
35. Kvakan H, Kleinewietfeld M, Qadri F, Park JK, Fischer R, Schwarz I, Rahn HP, Plehm R, Wellner M, Elitok S, Gratze P, Dechend R, Luft FC, Muller DN. Regulatory t cells ameliorate angiotensin ii-induced cardiac damage. Circulation. 2009;119:2904-2912

36. Coles B, Fielding CA, Rose-John S, Scheller J, Jones SA, O'Donnell VB. Classic interleukin-6 receptor signaling and interleukin-6 trans-signaling differentially control angiotensin ii-dependent hypertension, cardiac signal transducer and activator of transcription-3 activation, and vascular hypertrophy in vivo. Am J Pathol. 2007;171:315-325

37. Hilfiker-Kleiner D, Shukla P, Klein G, Schaefer A, Stapel B, Hoch M, Muller W, Scherr M, Theilmeier G, Ernst M, Hilfiker A, Drexler H. Continuous glycoprotein-130-mediated signal transducer and activator of transcription-3 activation promotes inflammation, left ventricular rupture, and adverse outcome in subacute myocardial infarction. Circulation. 2010;122:145-155

38. Kunisada K, Negoro S, Tone E, Funamoto M, Osugi T, Yamada S, Okabe M, Kishimoto T, Yamauchi-Takihara K. Signal transducer and activator of transcription 3 in the heart transduces not only a hypertrophic signal but a protective signal against doxorubicin-induced cardiomyopathy. Proc Natl Acad Sci U S A. 2000;97:315-319

39. Oshima Y, Fujio Y, Nakanishi T, Itoh N, Yamamoto Y, Negoro S, Tanaka K, Kishimoto T, Kawase I, Azuma J. Stat3 mediates cardioprotection against ischemia/reperfusion injury through metallothionein induction in the heart. Cardiovasc Res. 2005;65:428-435

40. Gao XM, Wong G, Wang B, Kiriazis H, Moore XL, Su YD, Dart A, Du XJ. Inhibition of mtor reduces chronic pressure-overload cardiac hypertrophy and fibrosis. J Hypertens. 2006;24:1663-1670

41. Gordon JW, Shaw JA, Kirshenbaum LA. Multiple facets of nf-\{kappa\}b in the heart: To be or not to nf-\{kappa\} b. Circ Res. 2011;108:1122-1132

42. Mann DL. The emerging role of innate immunity in the heart and vascular system: For whom the cell tolls. Circ Res. 2011;108:1133-1145

43. Bueno OF, Lips DJ, Kaiser RA, Wilkins BJ, Dai YS, Glascock BJ, Klevitsky R, Hewett TE, Kimball TR, Aronow BJ, Doevendans PA, Molkentin JD. Calcineurin abeta gene targeting predisposes the myocardium to acute ischemia-induced apoptosis and dysfunction. Circ Res. 2004;94:91-99

44. Bai L, Beckers L, Wijnands E, Lutgens SP, Herias MV, Saftig P, Daemen MJ, Cleutjens K, Lutgens E, Biessen EA, Heeneman S. Cathepsin $k$ gene disruption does not affect murine aneurysm formation. Atherosclerosis. 2010;209:96-103

45. Vandendriessche T, Thorrez L, Acosta-Sanchez A, Petrus I, Wang L, Ma L, L DEW, Iwasaki Y, Gillijns V, Wilson JM, Collen D, Chuah MK. Efficacy and safety of adeno-associated viral vectors based on serotype 8 and 9 vs. Lentiviral vectors for hemophilia b gene therapy. J Thromb Haemost. 2007;5:16-24

46. Swinnen M, Vanhoutte D, Van Almen GC, Hamdani N, Schellings MW, D'Hooge J, Van der Velden J, Weaver MS, Sage EH, Bornstein P, Verheyen FK, VandenDriessche T, Chuah MK, Westermann D, Paulus WJ, Van de Werf F, Schroen B, Carmeliet P, Pinto YM, Heymans S. Absence of thrombospondin-2 causes age-related dilated cardiomyopathy. Circulation. 2009;120:1585-1597

47. Junqueira LC, Bignolas G, Brentani RR. Picrosirius staining plus polarization microscopy, a specific method for collagen detection in tissue sections. Histochem J. 1979;11:447-455

48. Jorgensen S, Baker A, Moller S, Nielsen BS. Robust one-day in situ hybridization protocol for detection of micrornas in paraffin samples using Ina probes. Methods. 2010;52:375-381

49. Nuovo GJ. In situ detection of micrornas in paraffin embedded, formalin fixed tissues and the co-localization of their putative targets. Methods. 2010;52:307-315

50. De Windt LJ, Willemsen PH, Popping S, Van der Vusse GJ, Reneman RS, Van Bilsen M. Cloning and cellular distribution of a group ii phospholipase a2 expressed in the heart. J Mol Cell Cardiol. 1997;29:2095-2106

51. Peiser L, Gough PJ, Kodama T, Gordon S. Macrophage class a scavenger receptor-mediated phagocytosis of escherichia coli: Role of cell heterogeneity, microbial strain, and culture conditions in vitro. Infect Immun. 2000;68:1953-1963

52. Hume DA, Gordon S. Optimal conditions for proliferation of bone marrow-derived mouse macrophages in culture: The roles of csf-1, serum, ca2+, and adherence. J Cell Physiol. 1983;117:189-194 
53. Wiese M, Castiglione K, Hensel M, Schleicher U, Bogdan C, Jantsch J. Small interfering rna (sirna) delivery into murine bone marrow-derived macrophages by electroporation. J Immunol Methods. 2010;353:102-110

54. Yin X, Cuello F, Mayr U, Hao Z, Hornshaw M, Ehler E, Avkiran M, Mayr M. Proteomics analysis of the cardiac myofilament subproteome reveals dynamic alterations in phosphatase subunit distribution. Mol Cell Proteomics. 2010;9:497-509 


\title{
Supplementary Figures and Tables
}

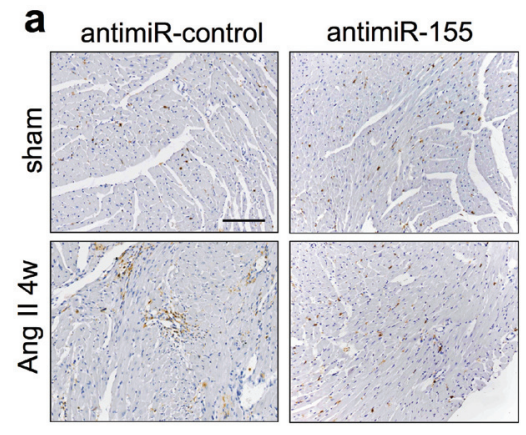

\author{
$\square$ antimiR-control a antimiR-155
}

b

$\mathrm{KO}$
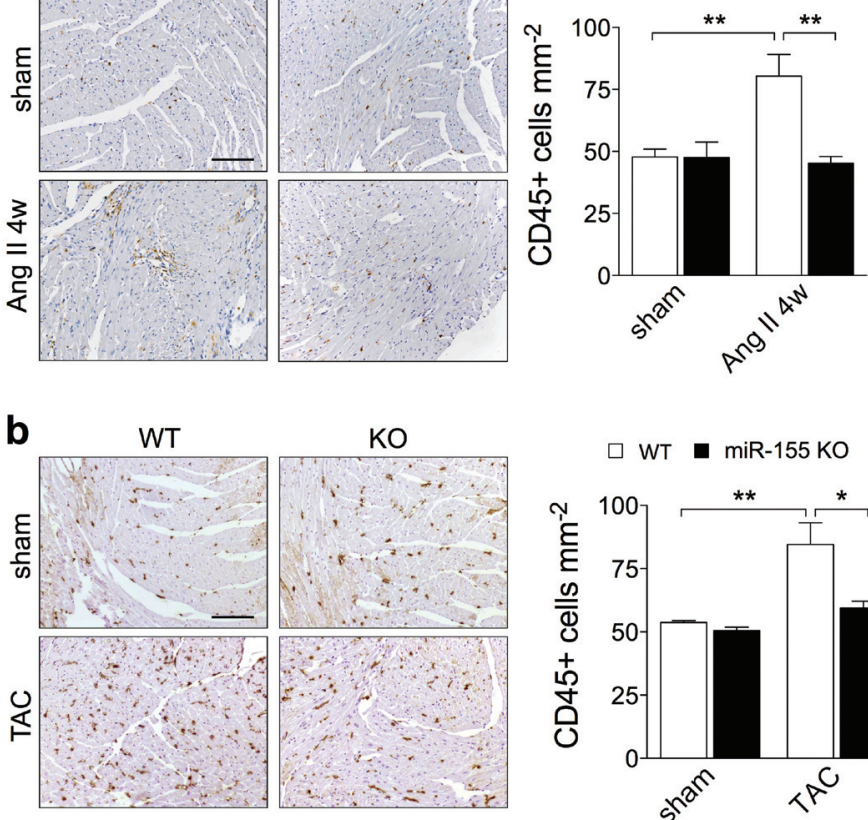

$\square$ WT $\square$ miR-155 KO

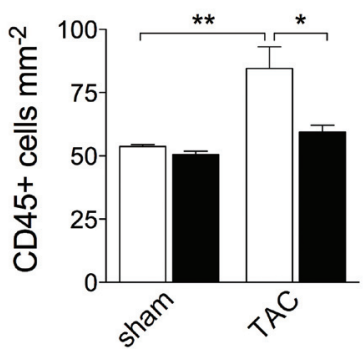

Supplementary Figure 1. MiR-155 absence reduces pressure overload-induced influx of inflammatory cells in the heart. (a) Quantification of immunoreactive cells for antibodies against CD45 (all leukocytes) in hearts of antagomiR-treated WT mice after Angll infusion shows blunting of cardiac infiltration by inflammatory cells in absence of miR-155. (b) Quantification of immunoreactive cells for antibodies against CD45 (all leukocytes) in miR-155 WT and KO hearts after TAC reveals blunting of cardiac infiltration by inflammatory cells in $\mathrm{KO}$ animals. Scale bars: $100 \mu \mathrm{m}$. All error bars represent s.e.m., ${ }^{*} \mathrm{P}<0.05,{ }^{*} \mathrm{P}<0.01$, ***P $<0.005$. 


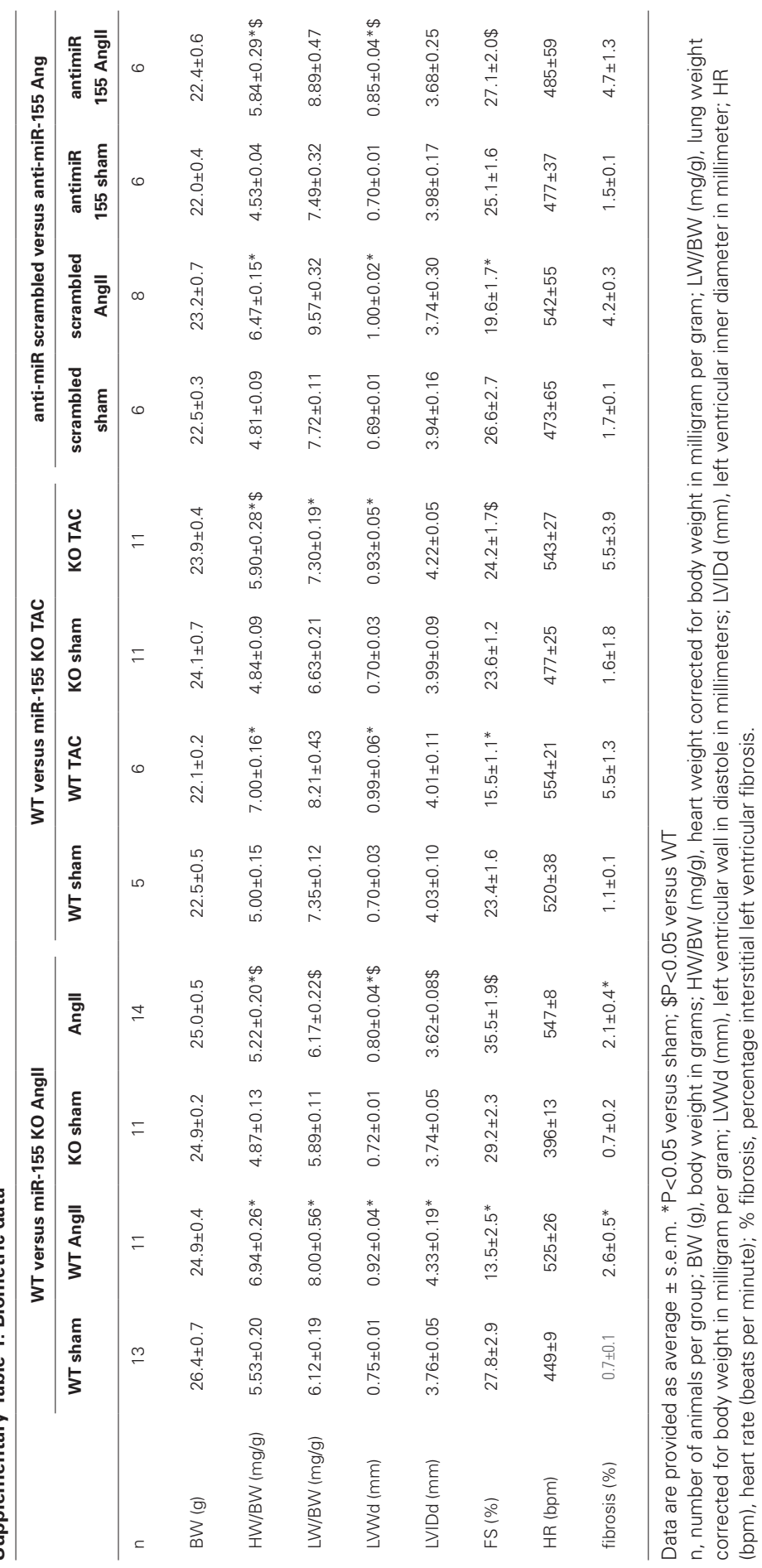


Supplementary Table 2. Peripheral blood flow cytometry in sham and Angll-treated miR-155 WT and KO mice

\begin{tabular}{|c|c|c|c|c|c|c|c|}
\hline & Marker & & & WT sham & WT Ang & KO sham & KO Ang \\
\hline \multirow[t]{4}{*}{ T cell } & CD3+ & & & $26.4 \pm 1.6$ & $24.8 \pm 2.8$ & $27.0 \pm 1.5$ & $25.8 \pm 1.3$ \\
\hline & & $\mathrm{CD} 4+^{1}$ & & $62.0 \pm 0.4$ & $54.3 \pm 0.7 \$ \$ \$$ & $57.8 \pm 0.9 * *$ & $55.7 \pm 1.0$ \\
\hline & & & $\begin{array}{l}\text { CD25+ } \\
\text { Foxp3+ }{ }^{2}\end{array}$ & $8.2 \pm 0.3$ & $10.2 \pm 0.4 \$ \$$ & $4.9 \pm 0.4^{* * *}$ & $5.5 \pm 0.3^{* * *}$ \\
\hline & & $\mathrm{CD} 8+{ }^{1}$ & & $35.2 \pm 0.4$ & $42.7 \pm 0.6 \$ \$ \$$ & $39.1 \pm 0.8^{* *}$ & $41.0 \pm 0.8$ \\
\hline B cell & B220+ & & & $30.7 \pm 4.2$ & $20.7 \pm 2.2$ & $36.9 \pm 3.8$ & $27.4 \pm 3.9$ \\
\hline \multirow[t]{2}{*}{ Monocyte } & $\begin{array}{l}\text { CD11b+ } \\
\text { Ly6G- }\end{array}$ & & & $11.9 \pm 0.8$ & $18.7 \pm 2.3 \$$ & $11.3 \pm 1.8$ & $11.4 \pm 1.0^{*}$ \\
\hline & & Ly6Chigh $^{3}$ & & $31.2 \pm 2.9$ & $45.0 \pm 2.2 \$ \$$ & $27.1 \pm 5.4$ & $35.6 \pm 4.6$ \\
\hline Granulocyte & $C D 11 b+L$ & $\mathrm{G}+$ & & $18.6 \pm 2.8$ & $29.7 \pm 2.0 \$$ & $19.1 \pm 2.1$ & $29.2 \pm 3.4 \$$ \\
\hline NK & NK1-1+ & & & $6.3 \pm 0.6$ & $6.8 \pm 1.4$ & $6.4 \pm 0.8$ & $5.4 \pm 0.3$ \\
\hline
\end{tabular}

Data are provided as average $\% \pm$ s.e.m. of life or parent; $n=6 / g r o u p ; 1 \%$ of $C D 3+; 2 \%$ of $C D 4+; 3 \%$ of CD11b+Ly6G-, ${ }^{*} P<0.05$, ${ }^{*} P<0.01,{ }^{* *} P<0.001$ versus $W T, \$ P<0.05, \$ \$ P<0.01, \$ \$ \$ P<0.001$ versus sham

Supplementary Table 3. Primers for quantitative real-time PCR

\begin{tabular}{lll}
\hline Gene & Forward primer & Reverse primer \\
\hline mmu-Acta1 & TGAGACCACCTACAACAGCA & CCAGAGCTGTGATCTCCTTC \\
mmu-Nppa & ATTGACAGGATTGGAGCCCAGAGT & TGACACACCACAAGGGCTTAGGAT \\
mmu-Nppb & GTTTGGGCTGTAACGCACTGA & GAAAGAGACCCAGGCAGAGTCA \\
mmu-II-6 & CAAAGCCAGAGTCCTTCAGAG & GCCACTCCTTCTGTGACTCC \\
mmu-GAPDH & GGTGGACCTCATGGCCTACA & CTCTCTTGCTCAGTGTCCTTGCT \\
rno-Acta1 & ATCACCAAGGGCTTCTTCCT & CCGCCGATCCACACTGA \\
rno-Nppa & ATCACCAAGGGCTTCTTCCT & TGTTGGACACCGCACTGTAT \\
rno-Nppb & GCTGCTTTGGGCAGAAGATAGA & GCCAGGAGGTCTTCCTAAAACA \\
rno-GAPDH & GGTGGACCTCATGGCCTACA & CTCTCTTGCTCTCAGTATCCTTGCT \\
\hline
\end{tabular}


Maarten F. Corsten ${ }^{1}$ MD

Robert Dennert ${ }^{1} \mathrm{MD}$

Sylvia Jochems ${ }^{1}$ BSc

Tatiana Kuznetsova ${ }^{2}$ MD PhD

Yvan Devaux ${ }^{3} \mathrm{PhD}$,

L. Hofstra ${ }^{4}$ MD PhD

Daniel R. Wagner ${ }^{3,5}$ MD PhD

Jan Staessen² MD PhD

Stephane Heymans* 1 MD PhD

Blanche Schroen ${ }^{*} 1 \mathrm{PhD}$

* Contributed equally

1 Center for Heart Failure Research, Cardiovascular Research Institute Maastricht, The Netherlands

2 Catholic University of Leuven, Department of Cardiovascular Diseases, Leuven, Belgium

3 Centre de Recherche Public (CRP) - Santé, Luxembourg, Luxembourg

4 Maastricht University Medical Center, Maastricht, The Netherlands

5 Centre Hospitalier Luxembourg, Luxembourg 


\section{Circulating MicroRNA-208b and MicroRNA-499 Reflect Myocardial Damage in Cardiovascular Disease}

Modified from Circulation Cardiovascular Genetics 2010;3:499-506 [IF 4.0]

\section{ABSTRACT}

Background Small RNA molecules, called microRNAs, freely circulate in human plasma and correlate with varying pathologies. In this study, we have explored their diagnostic potential in a selection of prevalent cardiovascular disorders.

Methods and Results MicroRNAs were isolated from plasmas from wellcharacterized patients with varying degrees of cardiac damage: a) acute myocardial infarction (AMI), b) viral myocarditis (VM), c) diastolic dysfunction, and d) acute heart failure (AHF). Plasma levels of selected microRNAs, including heart-associated (miR-1, -133a, -208b and -499), fibrosis-associated (miR-21 and miR-29b) and leukocyte-associated (miR-146, -155 and -223) candidates, were subsequently assessed using real-time PCR. Strikingly, in plasma from AMI patients, cardiac myocyte-associated miRs-208b and -499 were highly elevated, 1600-fold $(p<0.005)$ and 100 -fold $(p<0.0005)$ respectively, as compared with controls. ROC-curve analysis revealed an AUC of $0.94\left(p<10^{-10}\right)$ for miR-208b and $0.92\left(p<10^{-9}\right)$ for miR-499. Both microRNAs correlated with plasma troponin $\mathrm{T}$, indicating release of microRNAs from injured cardiomyocytes. In VM, we observed a milder but significant elevation of these microRNAs, 30- and 6-fold respectively. Plasma levels of leukocyte-expressed microRNAs were not significantly increased in AMI or VM patients, despite elevated white blood cell counts. In AHF patients, only miR-499 was significantly elevated (2-fold), while no significant changes in microRNAs studied could be observed in diastolic dysfunction. Remarkably, plasma microRNA levels were not affected by a wide range of clinical confounders, including age, gender, BMI, kidney function, systolic blood pressure, and white blood cell count.

Conclusions Cardiac damage initiates the detectable release of cardiomyocytespecific microRNAs-208b and -499 into the circulation. 


\section{Clinical perspective}

It was recently discovered that small RNAs, called microRNAs, circulate freely and stably in human plasma. This finding has sparked interest in the potential of microRNAs as biomarkers, because microRNAs are strongly implicated in cardiovascular disease and RNA molecules can be detected with high specificity and sensitivity using novel molecular techniques. We report profiles of microRNAs in varying cardiovascular disorders: acute myocardial infarction (AMI), acute viral myocarditis (VM), diastolic dysfunction and acute heart failure. Most strikingly, 2 cardiomyocyte-specific microRNAs (miR-208b and miR-499) are markedly elevated upon cardiac damage and correlate to circulating levels of troponin T. Our findings suggest a potential role for microRNAs as biomarkers in cardiology and mandate subsequent investigations to define their clinical applicability in early detection of myocardial damage. 


\section{Introduction}

MicroRNAs (miRNAs) form a class of small ( 22 nucleotides) non-coding RNAs that negatively regulate gene expression by recognizing complementary messenger RNAs (mRNAs) and prohibiting their translation into functional protein'. Their critical (patho) physiological importance is evidenced by their marked evolutionary conservation and current estimates are that they fin-tune expression of up to $50 \%$ of protein-coding genes ${ }^{2,3}$. MiRNAs are crucial for virtually all cellular processes and are a prerequisite for normal cardiac function ${ }^{4-7}$. Consequently, aberrant miRNA expression profiles are associated with various cardiovascular conditions such as hypertrophy, fibrosis, heart failure, and arrhythmias $^{8-11}$.

Recently, different groups reported that non-cell associated miRNAs are circulating freely in mammalian blood with marked biostability, and can be detected with high sensitivity and specificity in human plasma and serum ${ }^{12,13}$. Though the biological meaning of these miRNAs is unclear, pioneer profiling studies have attributed specific serum miRNA signatures to conditions varying from pregnancy to drug-induced liver injury, sepsis, and a variety of malignancies ${ }^{12-16}$. The diagnostic potential of miRNA detection in human plasma for cardiovascular disorders is beginning to be explored: Ai et al. studied plasmas of 93 AMI patients and found miR-1 increased ${ }^{17}$; Adachi et al. found elevated miR-499 in plasmas of 9 AMI patients ${ }^{18}$; increased plasma levels of miR-208a were reported by Wang et al. for $33 \mathrm{AMI}$ patients ${ }^{19}$; Cheng et al. reported increased plasma miR-1 levels in $31 \mathrm{AMI}$ patients ${ }^{20}$; and finally in a total of 42 heart failure patients, miR-423-5p was found increased by Tijsen et al. and correlated with proBNP levels ${ }^{21}$. Cardiovascular disease populations typically are elderly, overweight, and are associated with possible confounding factors such as age, gender, body-mass-index, and kidney and liver function. The importance of these clinical confounders for multiple plasma miRNA levels has, to our knowledge, not been investigated to date.

\section{Methods}

\section{Clinical samples}

All clinical samples were obtained with approval of the local Human Research Ethical Committees and stored at -80 degrees Celsius. They were subjected to freeze-thawing once.

\section{Acute myocardial infarction (AMI)}

Citrate plasma samples were obtained from patients presenting with $\mathrm{AMI}(n=32)$ and patients with atypical chest pain, positive stress testing but with normal coronary angiograms $(n=36)$. All patients with acute MI were enrolled in the Luxembourg acute MI registry and treated with primary percutaneous coronary intervention. All patients had successful mechanical reperfusion and stenting of the infarct artery within 12 hours of chest pain onset. All patients received Aspirin, Clopidogrel, Heparin and Abciximab. Acute MI was 
defined by the presence of chest pain $<12$ hours with significant ST elevation and increase in creatine kinase and troponin I to greater than 2-fold upper limit of normal. Blood samples were obtained at the time of mechanical reperfusion. More details are provided in Supplementary Table 1.

\section{Viral myocarditis (VM)}

Ethylenediaminetetraacetic acid (EDTA) plasma samples were obtained from patients with acute $V M$ during hospitalization in the acute phase $(n=14)$, post- $V M$ phase (ranging from 1 to 12 months after the acute inflammatory phase, $n=20$ ) and from age-matched healthy controls $(n=20)$ at the Cardiology Department of the Maastricht University Medical Center between 2007 and 2009. Acute VM patients were further subdivided into mild VM (troponin $\mathrm{T}<0.01 \mu \mathrm{g} / \mathrm{L}$ and normal left ventricular ejection fraction (LVEF), $\mathrm{n}=4$ ), moderate VM (elevated troponin $T$ or $\operatorname{LVEF}<50 \%, n=5)$ and severe $\operatorname{VM}(\operatorname{LVEF}<30 \%, n=5)$. See Supplementary Table 2.

\section{Diastolic dysfunction}

We examined EDTA plasma samples from 79 individuals at ages of 42-85, originally selected from a general Flemish population for the FLemisch Study on Enviromnent, Genes, and Health Outcomes (FLEMENGHO ${ }^{22}$ ), and thoroughly examined including echocardiographic analysis and detailed epidemiological characterization, as described previously ${ }^{23}$. Asmptomatic individuals were age-matched and divided into three groups. Group 1: controls $(n=20)$ with normal cardiac function and no risk factors, corresponding to AHA stage 0 of Heart Failure ${ }^{24}$. Group 2: hypertensive individuals $(n=20)$ without structural of functional cardiac involvement, corresponding to AHA stage A of HF. Group 3: hypertensive individuals with clear signs of cardiac involvement $(n=39)$ with impaired left ventricle $(L V)$ relaxation, or elevated end-diastolic LV pressures, corresponding the AHA stage B of HF. Full details are provided in Supplementary Table 3.

\section{Acute heart failure (AHF)}

EDTA plasma samples were collected from patients that were admitted with acute decompensation of congestive heart failure according to predefined Framingham-based criteria ${ }^{25}$ and an elevated plasma NT-proBNP (>200pmol/L) ( $n=33$ ), and from healthy staffmembers as controls $(n=34)$ at the Cardiology Department of the Maastricht University Medical Center. For additional details, see Supplementary Table 4.

\section{Whole blood samples}

To compare miRNA levels in matched plasma and leukocyte-rich whole blood, whole blood samples were used from 12 individuals from the Diastolic dysfunction cohort.

\section{Urine samples}

To investigate whether plasma miRs are excreted from the blood through glomerular filtration, simultaneous urine and EDTA plasma samples were taken from healthy volunteers $(n=5)$ from the VM healthy control group in Maastricht in November 2009. 


\section{RNA extraction}

Total RNA was extracted from $100 \mu \mathrm{L}$ of plasma, whole blood or urine, using the mirVana PARIS kit (Ambion, Warrington, UK), according to the manufacturer's instructions and without enrichment for small RNAs, and subsequently eluted in $50 \mu \mathrm{L}$ nuclease-free water. Subsequently, potential genomic DNA contamination was eliminated using DNA-free kit (Ambion).

\section{cDNA synthesis and $q R T-P C R$}

$15 \mu \mathrm{L}$ of RNA was used per $20-\mu \mathrm{L}$ reaction to generate cDNA using the miScript kit (Qiagen, Venlo, The Netherlands) that is designed to specifically detect mature microRNAs. The 20 $\mu \mathrm{L}$ reaction mix was then diluted $4 \mathrm{x}$ in nuclease free water and $2 \mu \mathrm{L}$ of cDNA was added per qPCR reaction, using BR SYBR-green supermix for IQ (Quanta Biosciences, Amsterdam, The Netherlands) in a MylQ iCycler (Bio-Rad, Veenendal, The Netherlands) device using MiScript primers sets (Qiagen). The selection of miRNA candidates to be tested was based on intellectual choice for each disease cohort. The following miRNAs were assayed: AMI: miR-1, -122, -133a, -208b, -223, -499; VM: miR-1, -133a, -146a, -146b, -155, -208b, -223, -499; Diastolic Dysfunction: miR-1, -21, -29b, -122, -126, -133a, -146a, -146b, -155, -208b, $-223,-499$ and AHF: miR-1, $-122,-133 a,-208 b,-223,-499$.

\section{Normalization of experimental data}

Quantitative qPCR measurements of miRNA levels were normalized for isolation efficiency using a mix of three spiked-in synthetic C. elegans miRNAs (Eurogentec, Maastricht, The Netherlands), lacking sequence homology to human miRNAs, as described previously ${ }^{13}$. Oligonucleotides were spiked into the samples during RNA isolation following plasma incubation with the provided $2 x$ denaturing solution. We have chosen to correct for spikes rather than for endogenous "stable" miRNAs, since there is no consensus in the literature regarding stable microRNAs for correction.

\section{Statistical analyses}

Statistical analyses were performed using GraphPad Prism 4.0a for Macintosh (GraphPad Software Inc., La Jolla, CA, USA),. Data are presented as mean \pm SEM unless indicated otherwise and plasma miRNA levels are presented as fold-change relative to controls. Linear regression analyses were used to correlate quantitative variables following normalization of non-Gaussian variables by log-transformation. Regression analyses of miRNA levels versus clinical parameters in the diastolic dysfunction database involved $\mathrm{n}=79$ patient plasmas, resulting in a power of $80 \%$ to demonstrate a Pearson correlation of 0.31. Comparisons between 2 groups were performed with Student's t tests for Gaussian data or Mann-Whitney tests for non-Gaussian data. For comparisons of more than 2 groups, one-way ANOVA was used, followed by post hoc testing using Bonferroni correction for more groups. Receiver operating characteristic curves were generated with PASW 18.0 for Macintosh, using nonparametric analyses to estimate error margins for the area under the curve. 


\section{Results}

\section{Plasma miRNA-208b and $\mathbf{- 4 9 9}$ levels are highly elevated after acute myocardial infarction and correlate with cardiac injury markers}

We first established miRNA plasma levels in AMI patients as compared to patients presenting with atypical chest pain and no cardiac disease. Heart-associated miRNAs, but not liver-specific miR-122 or leukocyte-associated miR-223, were elevated in AMI patients compared to controls (Figure 1). While miR-133a $(p<0.05)$ and miR-1 (non significant) levels were mildly increased, we found a robust elevation of plasma miR-208b and miR-499 levels by $\sim 1600$-fold $(p<0.005)$ and $\sim 100$-fold $(P<0.0005)$, respectively. Receiver operating characteristic $(\mathrm{ROC})$ curves generated for both miRNAs revealed an area under the curve of 0.944 for miR-208b $(95 \%$ confidence interval $=0.863-1.000$, $\mathrm{p}<10^{-10}$ ) and 0.918 for miR-499 (95\% confidence interval $=0.842-0.995, \mathrm{p}<10^{-9}$ ) (Figure 2). Subsequently, we assessed the correlation between plasma levels of miR-208b and miR-499 with serum levels of cardiac injury markers troponin T (Figure 2C and D) and creatine phosphokinase (CPK) in the AMI group (Supplementary Figure 1). Both miR-208b and miR-499 correlated significantly to troponin T and CPK levels, with the highest degree of correlation observed for miR-499 (CPK: regression coefficient $R=0.41, p<0.0001$; and troponin $\mathrm{T}: \mathrm{R}=0.69, \mathrm{p}<0.0001$ )

Intriguingly, miR-223 was mildly but significantly decreased in AMI patients as compared to controls. We found no correlation between miR-223 levels and Troponin T levels or WBC counts (Supplementary Figure 1). Wang et al. also report decreased miR-223 levels in patients with sepsis ${ }^{26}$. Future research will need to establish if there is a rationale for the diminished presence of this predominantly granulocyte-originating miRNA in inflammation-related pathologies.

\section{Cardiomyocyte-associated but not inflammatory microRNAs are significantly elevated in the acute stage of viral myocarditis}

We subsequently investigated plasma miRNA levels in patients with acute VM, patients in the post VM phase, and healthy controls. Again, levels of miR-208b and miR-499 were significantly elevated (30- and 6-fold, respectively, $p<0.01$ versus controls for both) during the acute phase of VM (Figure 3 and Supplementary Figure 2). Their expression levels significantly correlated with the severity of VM: subgroup analysis within the acute VM patients revealed normal values of miRs-208b and -499 in mild VM patients (with no troponin T elevation), and increasing levels for moderate and severe VM ( 20-fold and 50-fold higher, respectively, than control levels for miR-208b, and $\sim 5$-fold and 10-fold for miR-499) (Supplementary Figure 2). Of note, the more accentuated rise in miRNA plasma levels in AMI compared to VM paralleled the higher troponin T levels in AMI patients (Supplementary Tables 1 and 2). Levels of miR-1, -133a and leukocyte-associated miRs-146a, -146b, -155 and -223 were not significantly different between groups (Figure 3), despite significant leukocytosis in the acute VM group (Supplementary Table 2). 

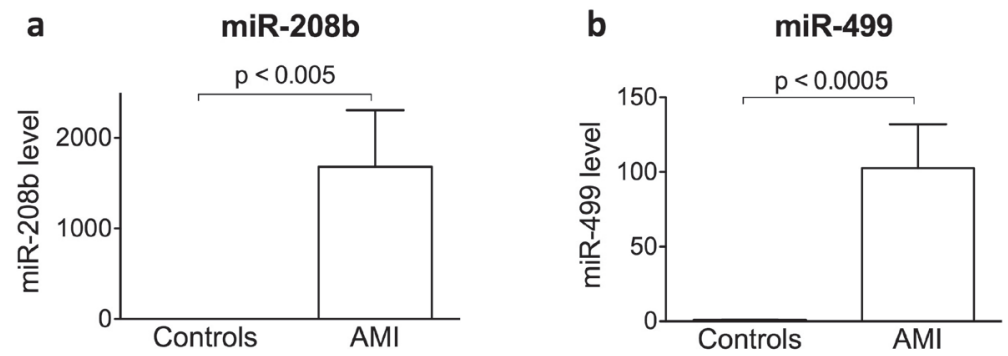

C

\section{miR-1}

d
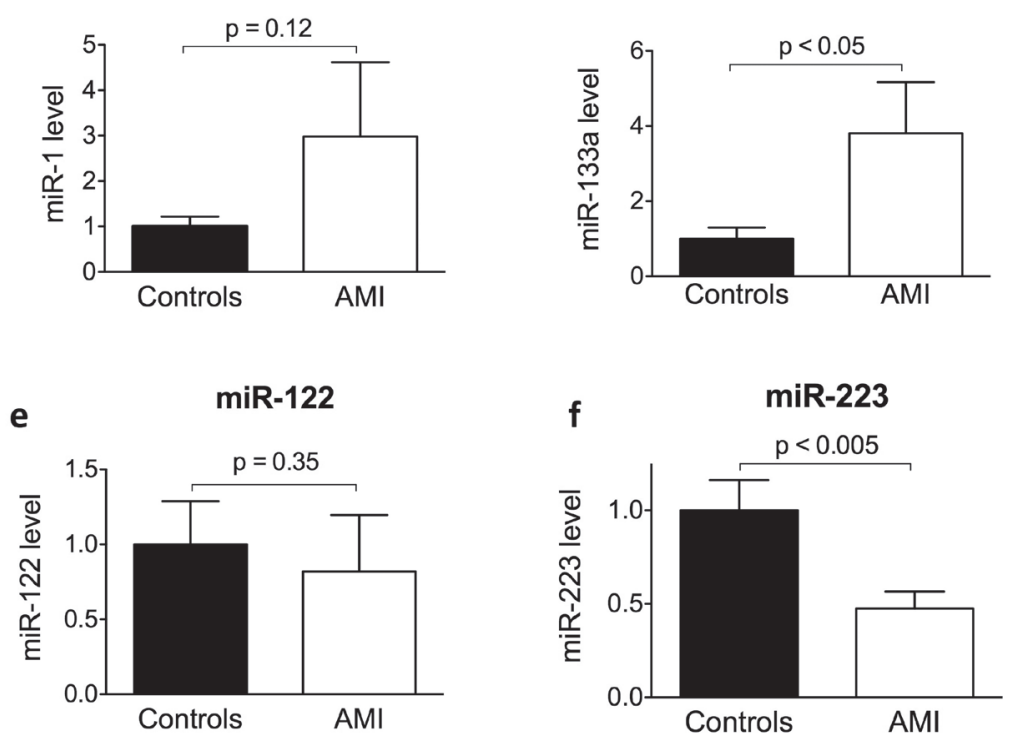

Figure 1. Cardiomyocyte miRNA levels mark acute myocardial infarction (AMI). Compared to microRNA levels in controls with atypical chest pain and no cardiac disease $(n=36)$, AMl patient plasmas $(n=32)$ show a sharp rise in levels of heart-associated microRNAs miR-208b (1600-fold, $p<0.005),-499$ (100 fold, $p<0,0005)$ and to a lesser extent miR-133a $(p<0.05)$ and miR-1 $(p=0.12)$ (panels A through D). Plasma levels of liverspecific miR-122 were not different between groups $(F)$ and miR-223 levels were even decreased in AMI plasmas $(p<0.005)(E)$.

\section{Cardiomyocyte- and leukocyte-expressed plasma miRNAs are not diagnostic for diastolic dysfunction}

None of the muscular or leukocytic miRNAs studied showed significantly different levels between the healthy controls, patients with hypertension without cardiac changes and patients with evident myocardial diastolic dysfunction (Figure 4 and Supplementary Figure 3). Also levels of miR-21 and -29b, which are both involved in the pathophysiology of cardiac fibrosis and upregulated in failing human hearts ${ }^{27-29}$, were not elevated in diastolic dysfunction plasmas. We then investigated correlations of the individual miRNA levels to 
cardiovascular indices. Intriguingly, the myocardium-associated miR-133a correlated significantly and positively to serum NT-proBNP levels ( $R=0.43, p=0.001$, see Figure $4 \mathrm{H}$ ), However, this correlation was independent of cardiac diastolic function, as miR-133a was not elevated in patients with diastolic dysfunction (Figure 4D). In addition, the NT-proBNP levels in this cohort were very mildly elevated, as opposed to the NT-proBNP levels in the acute heart failure cohort, where no correlation was found (Figure 5G).

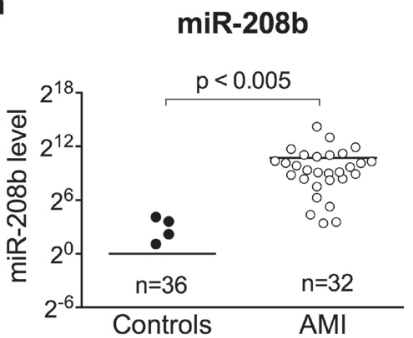

C Trop T vs miR-208b in AMI

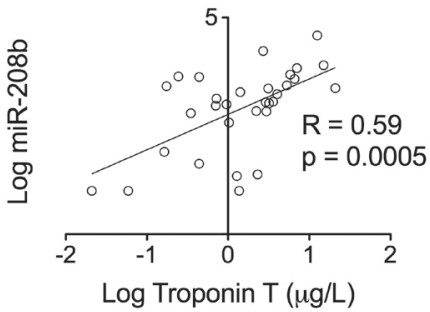

e ROC-curve miR-208b

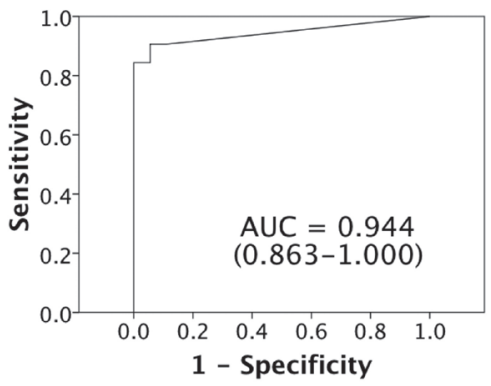

b

miR-499

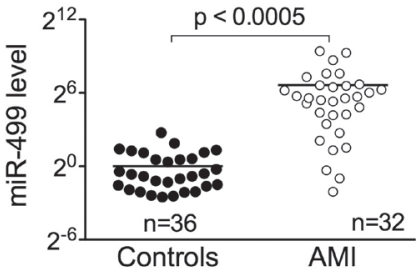

d Trop T vs miR-499 in AMI

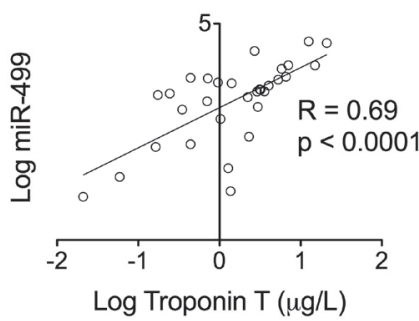

f ROC-curve miR-499

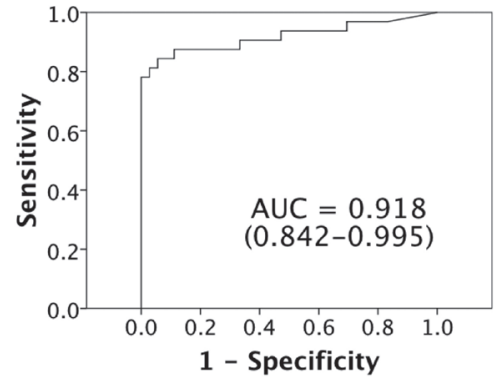

Figure 2. MiR-208b and miR-499 are potential circulating biomarkers for acute myocardial infarction (AMI). (A and B) Scatter plots of plasma miR levels in controls $(n=36)$ versus AMI patients $(n=32)$, revealing a 1500-fold increase for miR-208b $(p<0.005)$ and 90-fold increase for miR-499 $(p<0.001)$ in AMI. MiR-208b was not detected in 32 out of 36 controls and 3 our of $32 \mathrm{AMI}$ patients. (C and D) Within the AMI groups levels of miR-208b and miR-499 correlated significantly with circulating troponin T levels (miR-208b: R=0.59, $p=0.0005$ and miR-499: $R=0.69, p<0.0001)$. Panels A-D show that a few AMI patients had relatively low miR208 and miR-499 levels. This was reproducible and not due to technical issues. (E and F) Receiver operating characteristic (ROC) curves revealing an area under the curve of 0.944 for miR-208b (95\% confidence interval: 0.863-1.00, $\mathrm{p}<10-10$ ) and 0.918 for miR-499 (95\% Cl: 0.842-0.995, $\mathrm{p}<10-9$ ). 
Tijsen et al. also found no association between miR-133a plasma levels and NT-proBNP levels in heart failure patients ${ }^{21}$. No correlations were observed between miRNA plasma levels and systolic blood pressure, or left ventricular mass index (Supplementary Table 5). Finally, no differences were found in plasma microRNA levels of smokers and non-smokers, with exception of miR-29b, which was significantly higher in smokers $(p=0.02)$.

\section{a}

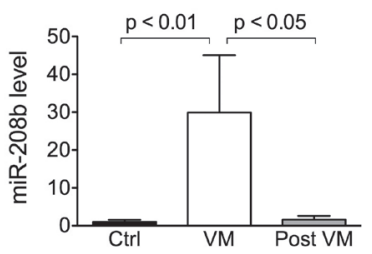

miR-1

C

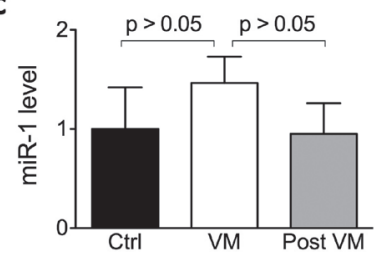

miR-146a

e

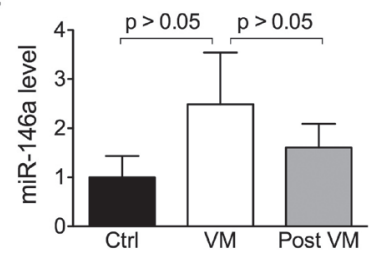

g

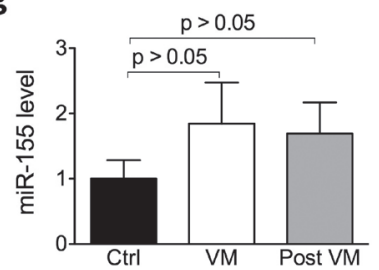

b

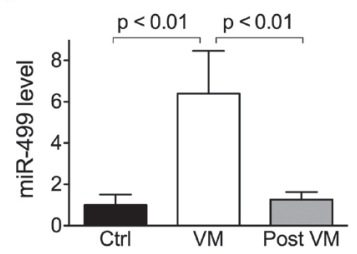

$\operatorname{miR}-133 a$

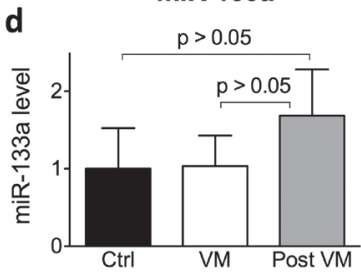

f

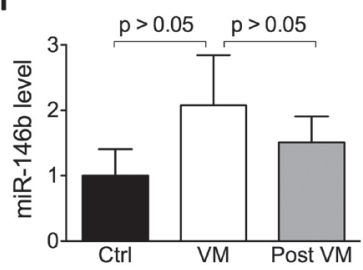

h $\quad$ miR-223

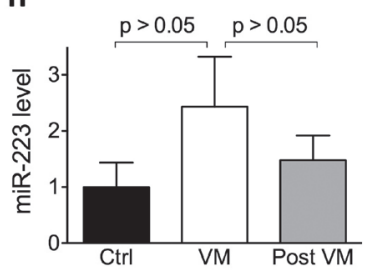

Figure 3. Plasma miRNA levels reflect myocardial damage but not inflammation in acute viral myocarditis. ( $A$ and B) MiR-208b and -499, which were the strongest indicators of myocardial damage in myocardial infarction, are significantly elevated in plasmas from acute VM patients $(n=14)$, compared to post VM patients ( $n=20, p<0.05$ for miR-208b and $p<0.01$ for miR-499) or healthy controls ( $n=20, p<0.01$ for both miRNAs). MiRs-1 and -133a levels were not significantly different between groups (C and D). (E-H) Leukocyte-associated miRs-146a, -146b, -155 , and -223 showed a non-significant trend to higher levels during the acute phase of VM. 
a

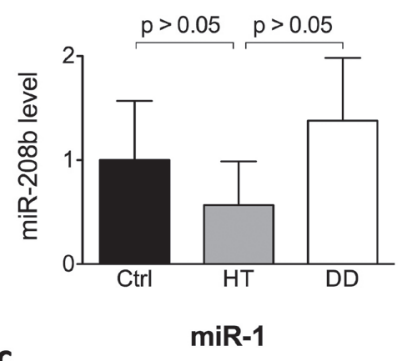

C

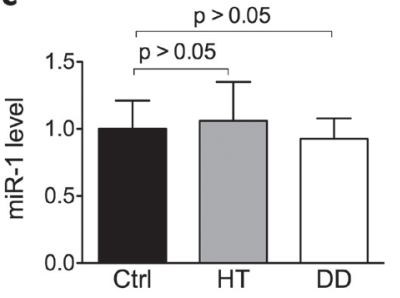

e

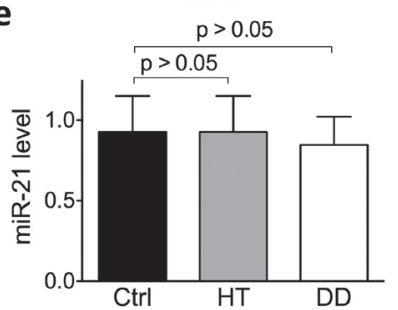

g

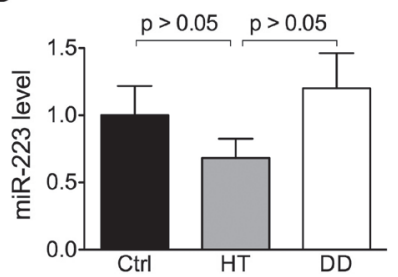

b

miR-499

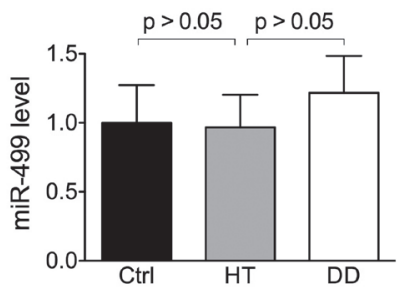

d

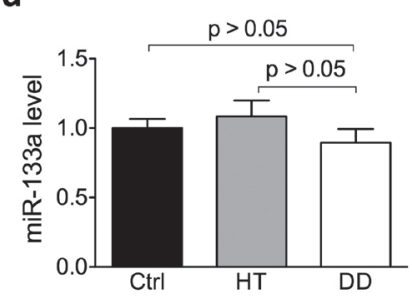

f

miR-29b

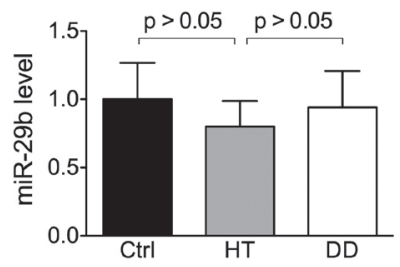

h NT-proBNP vs miR-133a

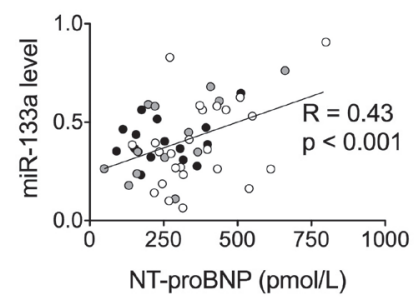

Figure 4. Plasma miRNA levels in a cohort of diastolic dysfunction. Levels of our plasma microRNA panel did not discriminate between diastolic dysfunction patients $(n=39)$ and hypertensive $(n=20)$ or normotensive $(n=20)$ controls. Panels A through $G$ depict levels of the muscle-associated miRs-208b, -499, -1 and -133 (A-D), fibrosis associated miRs-21 and $-29 b$ ( $E$ and F), and the leukocyte-associated miR-223 (G). $(\mathrm{H})$ The striated muscle associated miR-133a correlates significantly to serum N-terminal prohormone brain natriuretic peptide (NT-proBNP) levels in patients with diastolic dysfunction ( $R=0.43, p=0.001)$. Color-coding of data points in $4 \mathrm{H}$ is as follows: controls black, hypertensive patients grey, and patients with diastolic dysfunction white. 


\section{Acute heart failure provokes mild differences in heart-associated miRNAs}

We subsequently assessed plasma miRNA levels in patients with acutely decompensated AHF. All heart-associated miRNAs showed a trend towards mildly higher plasma levels in the AHF group, reaching significance for miR-499 (2-fold; $p<0.05$ ) (Figure 5). Of note, significance might have been masked for miR-1, by the mild negative correlation of miR-1 with age (Supplementary Table 1, $R=-0.25, p=0.04$ ) and the higher mean age of AHF patients versus controls. Additionally, the liver-specific miR-122, that is known to correlate with hepatic damage ${ }^{16}$, was significantly elevated in AHF patients (Figure 5), possibly reflecting hepatic venous congestion. In this patient group, we found no positive correlation of miR-133a with NT-pro-BNP levels (Figure 5G), nor with troponin T levels (Figure $5 \mathrm{H}$ ).

\section{Circulating plasma miRNAs are markedly robust against clinical confounders including age, gender, and renal function}

To determine to which extent plasma miRNA levels are confounded by the baseline characteristics of plasma donors, we selected 79 samples from the extensively characterized diastolic dysfunction patient database to evaluate the potential impact of a set of clinical confounders on miRNA levels (See Supplementary Table 5). Linear regression analyses revealed that plasma miRNA levels were not affected by the evaluated parameters. Patient gender and body mass index did not correlate with any of the plasma miRNAs (Supplementary Table 5). Plasma sample age, ranging from 11 to 49 months also had no influence on detected levels, indicating that miRNAs are not degraded when stored at -80 degrees Celsius. Patient age did not significantly correlate with 10 out of 12 miRNAs, and only showed weak negative correlation with miRNAs -1 and $-29 b(R=-0.25$ and -0.26 , respectively and $\mathrm{p}<0.05$ for both). Interestingly, glomerular filtration rate did not correlate with plasma miRNA levels. In addition, we investigated matched plasma and urine levels of miR-122 (liver) and -223 (granulocytes), and found that while both miRNAs were well detected in both sample types, levels in plasma compared to urine were 1000 -fold higher for miR-122 and 100 fold for miR-223 (Supplementary Figure 4). Together, these data indicate that freely circulating miRNAs are not renally cleared.

Plasma levels of the liver-specific miR-122 correlated with levels of the liver-specific marker gamma glutamyl transferase (gamma GT) (Supplementary Table 5), in agreement with the elevated levels of miR-122 in AHF (Figure 5E). Finally, levels of miR-133a correlated with NT-proBNP levels in this patient group, as described above.

\section{Leukocyte-associated miRNAs do not reflect white blood cell count or leukocytic miRNA levels}

To investigate whether leukocyte-associated miRNAs could serve as markers for mild inflammatory processes, we compared plasma levels of inflammatory miRNAs to WBC counts. Plasma levels did not correlate with circulating numbers of leukocytes, indicating that secretion of miRNAs from circulating leukocytes is not the main determinant of their plasma levels (Supplementary Figure 5 and Supplementary Table 5). This lack of correlation was independently confirmed in our plasma databases of acute VM and AMI plasmas with leukocytosis (Supplementary Figure 1 and data not shown). We subsequently compared 
a

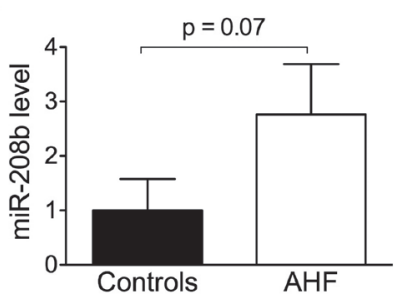

C

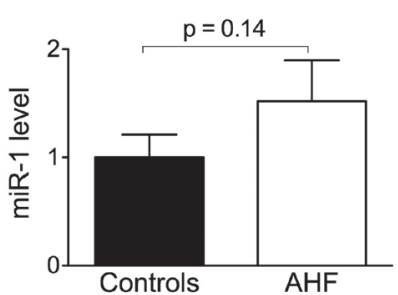

e

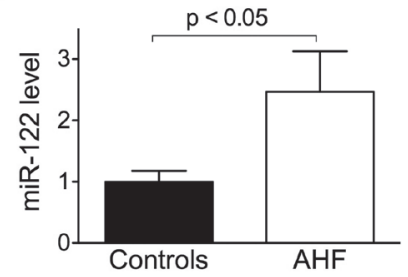

g

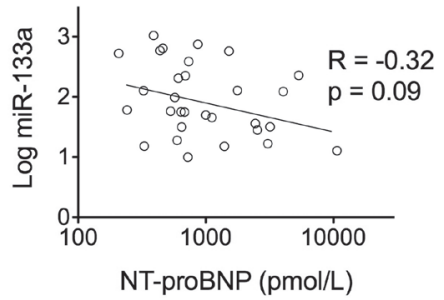

b

miR-499

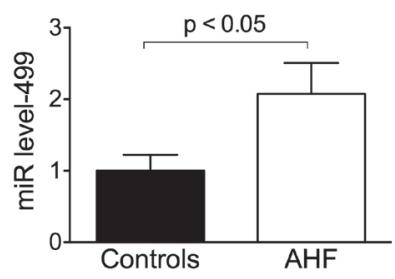

miR-133a

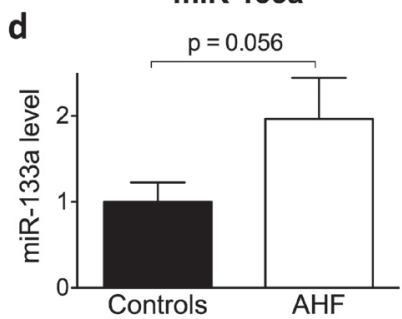

f

miR-223

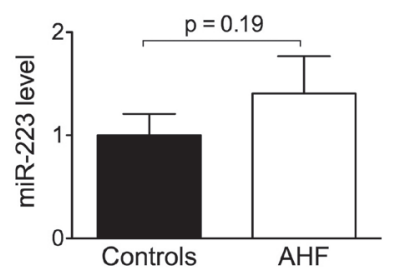

h Trop T versus miR-133a



Figure 5. Plasma miRNA levels in patients with acutely heart failure AHF. (A-D) Plasma levels of the heart-associated miRs-208b, -499, -1, and -133a were higher in AHF patients $(n=33)$ than in controls $(n=20)$, although only miR-499 level differences were significantly elevated $(p<0.05)$. The liver-specific miR-122 was elevated in AHF patients (E), while granulocyte-specific miR-223 was not different for AHF patients and controls (F). (G) In AHF patients, miR-133a failed to correlate with NT-proBNP (R=-0.32, $p=0.09$ ) as was observed in diastolic dysfunction patients. This lack of correlation could not be explained by a correlation with circulating troponin $T$ levels $(R=0.27, p=0.23)(H)$. 
the levels of plasma miRNA with whole blood miRNA levels (containing leukocytes) across patients, but again did not find a significant correlation (Supplementary Figure 4), indicating that plasma miRNAs reflect neither WBC count nor leukocytic miRNA expression. However, we did notice a striking quantitative correlation between leukocyte-associated but not cardiac miRNAs within samples (Supplementary Table 6A and B), pointing towards a common etiology. We also found highly identical patterns of these miRNAs in plasma and whole blood (Supplementary Figure 6) within individuals, with levels always 1000-fold higher in whole blood, suggesting that leukocytes are indeed the primary source of these miRNAs in plasma. The paradoxical lack of correlation with WBC counts and whole blood miRNA might be explained by variable leakage between patients, compromising their diagnostic accuracy.

\section{Discussion}

Recent evidence that freely circulating miRNAs may be informative of human pathology has ignited wide interest in their diagnostic potential ${ }^{13,16}$. Our study demonstrates that in humans, diverse conditions of myocardial damage are associated with striking perturbations of plasma levels of heart-associated miRNAs-208b and -499. These are increasingly elevated during acute heart failure (minimal), viral myocarditis (marked), and acute myocardial infarction (extensive). In AMI, where cardiac damage was most severe, ROC-curves for AMI detection by miR-208b or -499 levels revealed a pronounced diagnostic accuracy, evidenced by an area under the curve of $>0.91$ with $p<10^{-9}$ for both individual miRNAs. Encouragingly, these data are in agreement with a recent report published during the preparation of this manuscript, that examined plasma levels of muscle-specific miRNAs in AMI patients and also identified miRs-208(a) and -499 as the most sensitive biomarkers of AMI ${ }^{19}$. Supplementary Table 7 provides an overview of the plasma miRNA changes reported in cardiovascular disease thus far. In addition, our study uniquely shows that miR-208b and -499 release is not exclusive for AMI and occurs independently of ischemia and ejection fraction. Biologically, these data evoke tantalizing questions about potential functions for circulating miR-208b and -499 , which modulate cardiomyocyte performance by shifting the balance between slow and fast muscle fibre gene programs towards the slow type ${ }^{7,30}$. There is currently no evidence demonstrating a functional role for plasma miRNAs through affecting distant gene expression in vivo. Interestingly, it was reported that following druginduced liver injury, miRNAs elevated in plasma were consistently downregulated in corresponding livers ${ }^{16}$ suggesting the intriguing possibility of a cellular survival mechanism in which, during stress, undesirable miRNAs are actively excreted. However, miR-208 is elevated in acutely infarcted human hearts ${ }^{31}$ and additional research is required to address the biological role of plasma miRNAs after cardiac damage.

Interestingly, the profound increases of miRNAs -208b and -499 in patients with AMI (1600and 100-fold, respectively) are in contrast with the mild elevations observed for heartassociated miRs-1 and -133a (3- and 4-fold, respectively). This could be a reflection of the higher specificity of miR-208b and -499 for cardiac muscle, or higher baseline levels of 
miR-1 and -133a in serum, due to turnover of skeletal muscle ${ }^{19}$. An alternative possibility is that these miRNAs might be trapped in structural complexes in cardiomyocytes, preventing leakage. Three groups previously reported that plasma miR-1 levels are elevated in AMI patients ${ }^{17}, 19,20$, but also in these studies this elevation was milder than observed for miR-208b and -499. Our data generally support their conclusions but extend on their findings by identifying miRs $-208 \mathrm{~b}$ and -499 as far more sensitive markers for myocardial damage not only in AMI but also in VM. An intruiging observation from our study was the correlation of miR-133a plasma levels with with NT-proBNP in asymptomatic patients with diastolic dysfunction, which was not observed in AHF patients. Additional research in larger patient populations will be required to unveil the significance of this phenomenon.

The influence of potentially confounding patient characteristics has so far remained largely unexplored. We present evidence that the investigated plasma miRNA levels are highly robust to a variety of patient parameters including age, gender, body mass index, systolic blood pressure, smoking, and glomerular filtration rate. Further, the liver-specific miR-122 was the only miRNA mildly correlating with gamma GT levels, in agreement with the recent report that plasma miR-122 parallels levels of aminotransferase levels during hepatic damage $^{16}$. These findings are of vital importance for the diagnostic applicability of plasma miRNAs in clinical settings, and in particular cardiovascular medicine, where skewed distributions of age and biometry, combined with comorbidities such as renal failure are common. Our data indicate caution to use leukocyte-expressed plasma miRNAs as diagnostic targets in conditions of mild inflammation, as their levels may be subject to variable leakage from circulating leukocytes, eg caused by venipuncture. On the other hand, the significant decrease in miR-223 levels following AMI has potential biological and/ or diagnostic significance, given the study by Wang et al. ${ }^{26}$ who find decreased miR-223 levels during sepsis. Therefore, the value of miR-223 for AMI needs to be investigated further.

In conclusion, we report that cardiac damage in diverse cardiac diseases initiates massive release of cardiomyocyte-specific microRNAs into the circulation, and that these microRNAs are highly insensitive to clinical characteristics in a cardiovascular patient population. 


\section{References}

1. Bartel DP. MicroRNAs: genomics, biogenesis, mechanism, and function. Cell. 2004;116:281-297.

2. Baek D, Villen J, Shin C, Camargo FD, Gygi SP, Bartel DP. The impact of microRNAs on protein output. Nature. 2008;455:64-71.

3. Selbach M, Schwanhausser B, Thierfelder N, Fang Z, Khanin R, Rajewsky N. Widespread changes in protein synthesis induced by microRNAs. Nature. 2008;455:58-63.

4. Chien KR. Molecular medicine: microRNAs and the tell-tale heart. Nature. 2007;447:389-390.

5. da Costa Martins PA, Bourajjaj M, Gladka M, Kortland M, van Oort RJ, Pinto YM, Molkentin JD, De Windt LJ. Conditional dicer gene deletion in the postnatal myocardium provokes spontaneous cardiac remodeling. Circulation. 2008;118:1567-1576.

6. Liu N, Bezprozvannaya S, Williams AH, Qi X, Richardson JA, Bassel-Duby R, Olson EN. microRNA-133a regulates cardiomyocyte proliferation and suppresses smooth muscle gene expression in the heart. Genes Dev. 2008;22:3242-3254.

7. van Rooij E, Quiat D, Johnson BA, Sutherland LB, Qi X, Richardson JA, Kelm RJ, Jr., Olson EN. A family of microRNAs encoded by myosin genes governs myosin expression and muscle performance. Dev Cell. 2009; 17:662-673.

8. Care A, Catalucci D, Felicetti F, Bonci D, Addario A, Gallo P, Bang ML, Segnalini P, Gu Y, Dalton ND, Elia L, Latronico MV, Hoydal M, Autore C, Russo MA, Dorn GW, 2nd, Ellingsen O, Ruiz-Lozano P, Peterson KL, Croce CM, Peschle C, Condorelli G. MicroRNA-133 controls cardiac hypertrophy. Nat Med. 2007;13:613-618.

9. Schroen B, Heymans S. MicroRNAs and beyond: the heart reveals its treasures. Hypertension. 2009;54:11891194.

10. Thum T, Galuppo P, Wolf C, Fiedler J, Kneitz S, van Laake LW, Doevendans PA, Mummery CL, Borlak J, Haverich A, Gross C, Engelhardt S, Ertl G, Bauersachs J. MicroRNAs in the human heart: a clue to fetal gene reprogramming in heart failure. Circulation. 2007;116:258-267.

11. van Rooij E, Sutherland LB, Liu N, Williams AH, McAnally J, Gerard RD, Richardson JA, Olson EN. A signature pattern of stress-responsive microRNAs that can evoke cardiac hypertrophy and heart failure. Proc Natl Acad Sci U S A. 2006;103:18255-18260.

12. Gilad S, Meiri E, Yogev Y, Benjamin S, Lebanony D, Yerushalmi N, Benjamin H, Kushnir M, Cholakh H, Melamed N, Bentwich Z, Hod M, Goren Y, Chajut A. Serum microRNAs are promising novel biomarkers. PLoS One. 2008;3:e3148.

13. Mitchell PS, Parkin RK, Kroh EM, Fritz BR, Wyman SK, Pogosova-Agadjanyan EL, Peterson A, Noteboom J, O'Briant KC, Allen A, Lin DW, Urban N, Drescher CW, Knudsen BS, Stirewalt DL, Gentleman R, Vessella RL, Nelson PS, Martin DB, Tewari M. Circulating microRNAs as stable blood-based markers for cancer detection. Proc Natl Acad Sci U S A. 2008;105:10513-10518.

14. Chen X, Ba Y, Ma L, Cai X, Yin Y, Wang K, Guo J, Zhang Y, Chen J, Guo X, Li Q, Li X, Wang W, Wang J, Jiang X, Xiang Y, Xu C, Zheng P, Zhang J, Li R, Zhang H, Shang X, Gong T, Ning G, Zen K, Zhang CY. Characterization of microRNAs in serum: a novel class of biomarkers for diagnosis of cancer and other diseases. Cell Res. 2008;18:997-1006.

15. Vasilescu C, Rossi S, Shimizu M, Tudor S, Veronese A, Ferracin M, Nicoloso MS, Barbarotto E, Popa M, Stanciulea O, Fernandez MH, Tulbure D, Bueso-Ramos CE, Negrini M, Calin GA. MicroRNA fingerprints identify miR-150 as a plasma prognostic marker in patients with sepsis. PLoS One. 2009;4:e7405.

16. Wang K, Zhang S, Marzolf B, Troisch P, Brightman A, Hu Z, Hood LE, Galas DJ. Circulating microRNAs, potential biomarkers for drug-induced liver injury. Proc Natl Acad Sci U S A. 2009;106:4402-4407.

17. Ai J, Zhang R, Li Y, Pu J, Lu Y, Jiao J, Li K, Yu B, Li Z, Wang R, Wang L, Li Q, Wang N, Shan H, Yang B. Circulating microRNA-1 as a potential novel biomarker for acute myocardial infarction. Biochem Biophys Res Commun. 2009;391:73-77.

18. Adachi T, Nakanishi M, Otsuka Y, Nishimura K, Hirokawa G, Goto Y, Nonogi H, Iwai N. Plasma microRNA 499 as a biomarker of acute myocardial infarction. Clin Chem. 2010;56:1183-1185. 
19. Wang GK, Zhu JQ, Zhang JT, Li Q, Li Y, He J, Qin YW, Jing Q. Circulating microRNA: a novel potential biomarker for early diagnosis of acute myocardial infarction in humans. Eur Heart J. 2010;31:659-666.

20. Cheng Y, Tan N, Yang J, Liu X, Cao X, He P, Dong X, Qin S, Zhang C. A translational study of circulating cell-free microRNA-1 in acute myocardial infarction. Clin Sci (Lond). 2010;119:87-95.

21. Tijsen AJ, Creemers EE, Moerland PD, de Windt LJ, van der Wal AC, Kok WE, Pinto YM. MiR423-5p as a circulating biomarker for heart failure. Circ Res. 2009;106:1035-1039.

22. Zhang H, Thijs L, Kuznetsova T, Fagard RH, Li X, Staessen JA. Progression to hypertension in the nonhypertensive participants in the Flemish Study on Environment, Genes and Health Outcomes. J Hypertens. 2006;24:1719-1727.

23. Kuznetsova T, Herbots L, Lopez B, Jin Y, Richart T, Thijs L, Gonzalez A, Herregods MC, Fagard RH, Diez J, Staessen JA. Prevalence of left ventricular diastolic dysfunction in a general population. Circ Heart Fail. 2009;2:105-112.

24. Jessup M, Abraham WT, Casey DE, Feldman AM, Francis GS, Ganiats TG, Konstam MA, Mancini DM, Rahko PS, Silver MA, Stevenson LW, Yancy CW. 2009 focused update: ACCF/AHA Guidelines for the Diagnosis and Management of Heart Failure in Adults: a report of the American College of Cardiology Foundation/American Heart Association Task Force on Practice Guidelines: developed in collaboration with the International Society for Heart and Lung Transplantation. Circulation. 2009;119:1977-2016.

25. McKee PA, Castelli WP, McNamara PM, Kannel WB. The natural history of congestive heart failure: the Framingham study. N Engl J Med. 1971;285:1441-1446.

26. Wang JF, Yu ML, Yu G, Bian JJ, Deng XM, Wan XJ, Zhu KM. Serum miR-146a and miR-223 as potential new biomarkers for sepsis. Biochem Biophys Res Commun. 2010;394:184-188.

27. Matkovich SJ, Van Booven DJ, Youker KA, Torre-Amione G, Diwan A, Eschenbacher WH, Dorn LE, Watson MA, Margulies KB, Dorn GW, 2nd. Reciprocal regulation of myocardial microRNAs and messenger RNA in human cardiomyopathy and reversal of the microRNA signature by biomechanical support. Circulation. 2009;119:1263-1271.

28. Thum T, Gross C, Fiedler J, Fischer T, Kissler S, Bussen M, Galuppo P, Just S, Rottbauer W, Frantz S, Castoldi M, Soutschek J, Koteliansky V, Rosenwald A, Basson MA, Licht JD, Pena JT, Rouhanifard SH, Muckenthaler MU, Tuschl T, Martin GR, Bauersachs J, Engelhardt S. MicroRNA-21 contributes to myocardial disease by stimulating MAP kinase signalling in fibroblasts. Nature. 2008;456:980-984.

29. van Rooij E, Sutherland LB, Thatcher JE, DiMaio JM, Naseem RH, Marshall WS, Hill JA, Olson EN. Dysregulation of microRNAs after myocardial infarction reveals a role of miR-29 in cardiac fibrosis. Proc Natl Acad Sci U S A. 2008;105:13027-13032

30. Miyata S, Minobe W, Bristow MR, Leinwand LA. Myosin heavy chain isoform expression in the failing and nonfailing human heart. Circ Res. 2000;86:386-390.

31. Bostjancic E, Zidar N, Stajer D, Glavac D. MicroRNAs miR-1, miR-133a, miR-133b and miR-208 are dysregulated in human myocardial infarction. Cardiology. 2009;115:163-169. 


\section{Supplementary Figures and Tables}

a

CPK versus miR-208b in AMI

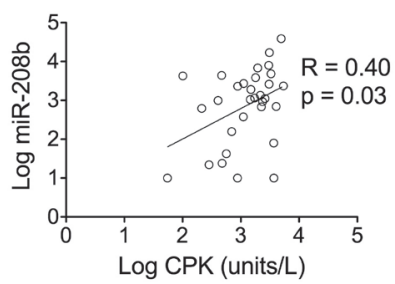

c

WBC count vs miR-223 in AMI

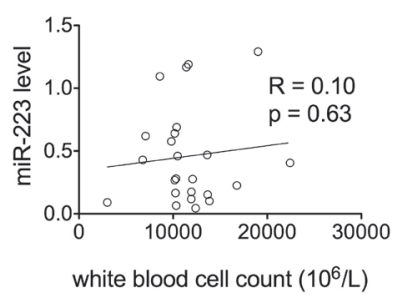

CPK versus miR-499 in AMI

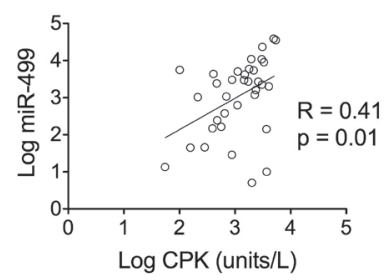

d

Trop T vs miR-223 in AMI

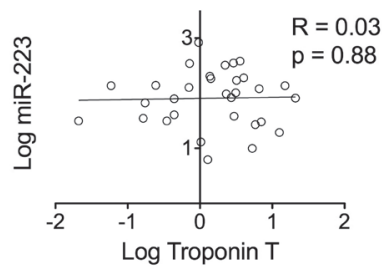

Supplementary Figure 1. Plasma miRNA levels in AMI. Plasma levels of miR-208b (panel A) and miR-499 (B) correlate with circulating levels of cardiac damage marker creatine phosphokinase (CPK) in AMI patients (miR-208b: $R=0.40, p=0.03$ and miR-499: $R=0.41, p=0.01$ ). Regression analysis between plasma levels of the granulocyte-specific miR-223 and white blood cell count (panel C) revealed no correlation $(R=0.10$, $p=0.63$ ). MiR-223 levels also did not correlate with levels of troponin $T$ (panel $D ; R=0.03, p=0.88$ ).
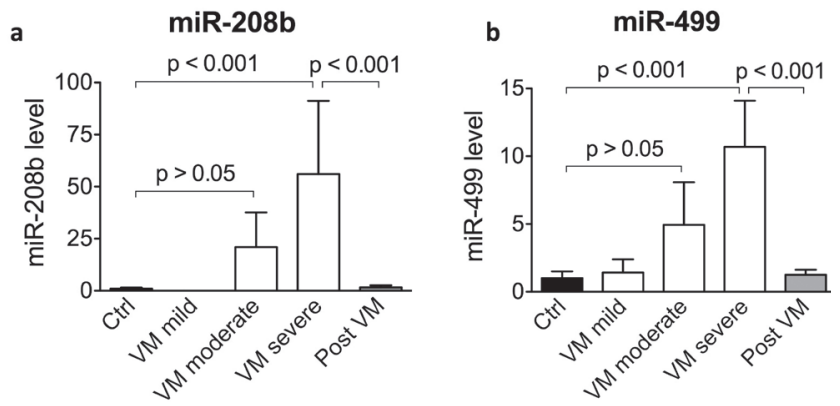

Supplementary Figure 2. Plasma miR-208b and miR-499 elevation in viral myocarditis reflects VM severity. Plasma levels of miR-208b (panel A) and miR-499 (panel B) are elevated in acute viral myocarditis in relation to disease severity, as classified into mild VM (troponin $T<0.01 \mu \mathrm{g} / \mathrm{L}$ and normal left ventricular ejection fraction (LVEF), $n=4$ ), moderate VM (elevated troponin $T$ or $L V E F<50 \%, n=5$ ) and severe VM (LVEF $<30 \%, n=5)$. MiR-208b was detected in only 4 out of 20 control patients and none of the patients in the mild VM group (that per definition does not include patients with troponin T elevation). MiR-499 was detected in all patients and levels were comparably low in the control and mild $\mathrm{V}$ group. Moderate VM samples display a trend towards elevation of both microRNAs and in severe VM, both miR-208b and miR499 are significantly elevated $(p<0.001)$. 
a

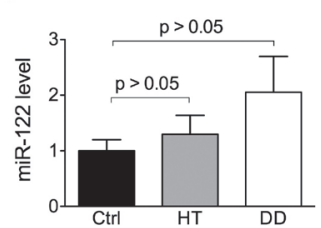

d

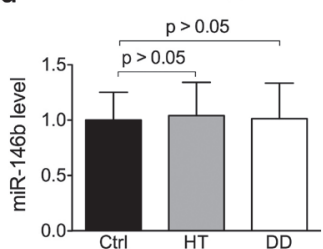

b

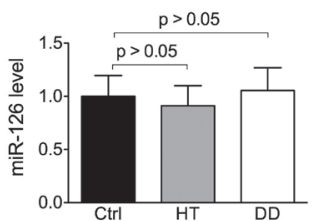

e

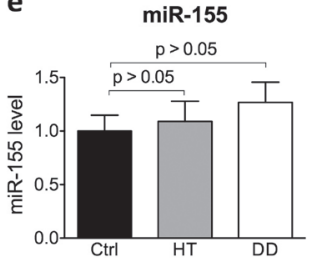

C

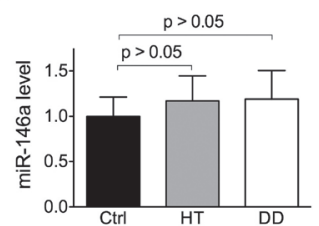

$\mathbf{f}$

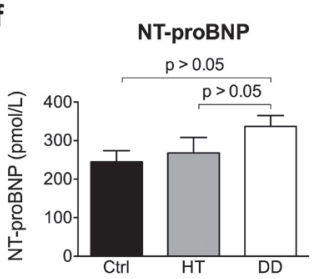

Supplementary Figure 3. Plasma miRNA levels in patients with myocardial Diastolic Dysfunction. Panels A through $F$ depict plasma miRNA levels in controls without hypertension or cardiovascular risk factors, hypertensive patients without cardiac involvement, and patients with evident diastolic dysfunction. MiRNAs studies included miRNAs associated with liver (miR-122), endothelium (miR-126), and leukocytes (miRs-146a, 146b, -155, 223). Panel F depicts NT-proBNP levels for all three groups (not significantly different).

\title{
plasma vs urine miRNA levels
}

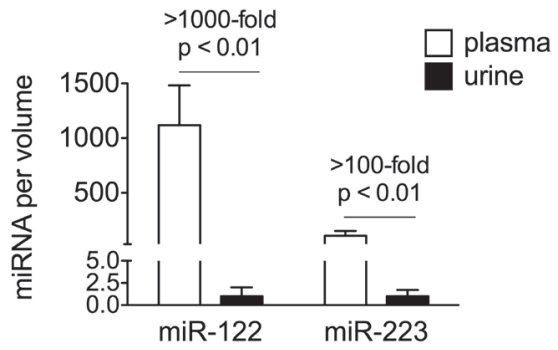

\begin{abstract}
Supplementary Figure 4. Plasma miRNAs are not subject to efficient glomerular filtration. MicroRNA detection in matched plasma and urine samples revealed substantially higher levels in plasmas for all investigated microRNAs, which, together with he lack of correlation of with glomerular filtration rate, indicates that microRNAs are not renally cleared. Displayed microRNAs were selected for predominant preglomerular origin (miR122: liver-specific; miR-223: granulocyte-specific). Error bars represent standard deviations.
\end{abstract}


a

miR-146b

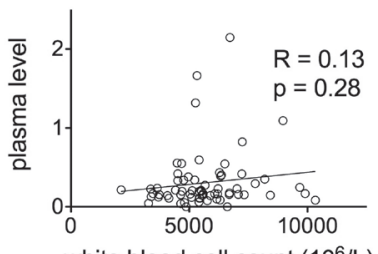

white blood cell count $\left(10^{6} / \mathrm{L}\right)$

C

\section{miR-155}

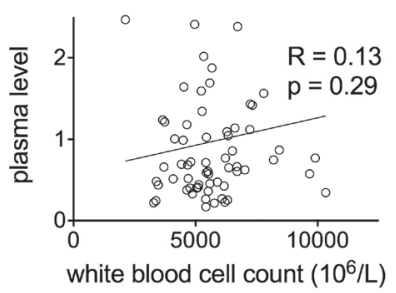

e

miR-223

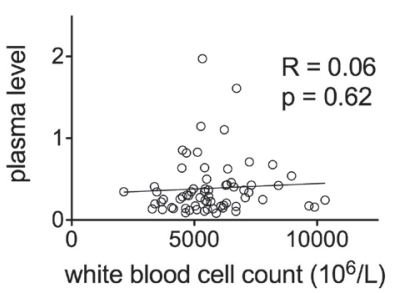

b

miR-146b

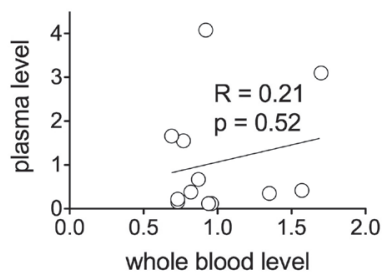

d

miR-155

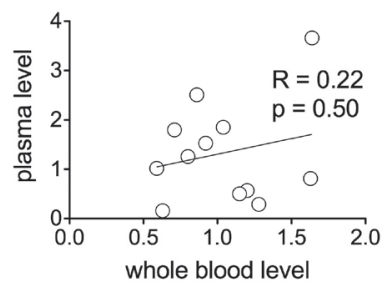

miR-223

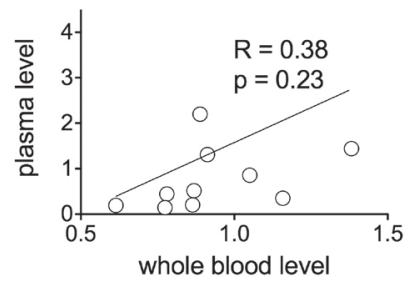

Supplementary Figure 5. Plasma microRNAs do not reflect white blood cell count or leukocytic microRNA fraction in Diastolic Dysfunction. Plasma levels of leukocyte-associated microRNAs-146a, -155 and -223 fail to correlate with circulating white blood cell counts ( $n=79$ ) (A, C and E). Plasma levels of leukocyte-associated microRNAs additionally do not correlate with measured microRNA levels from whole blood ( $n=12)(B, D$ and F). 
a

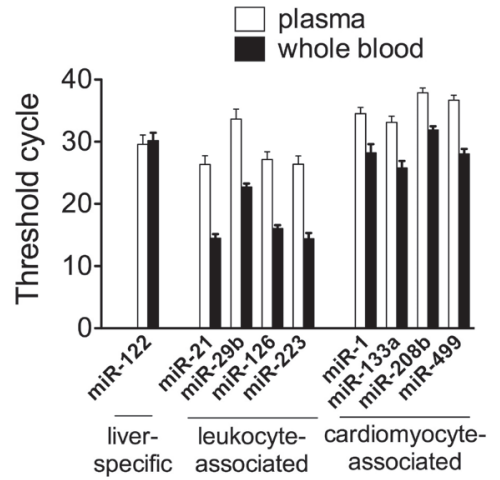

b

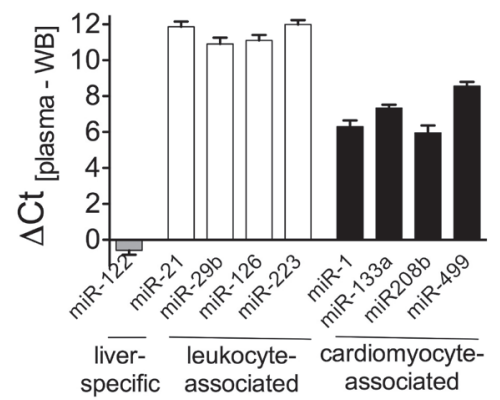

Supplementary Figure 6. Plasma microRNA profiles mirror microRNA profiles in the leukocyte blood fraction and might be skewed by leakage. (A) Average threshold cycle (Ct) values for qRT-PCR detection of microRNAs in paired plasma and whole blood samples $(n=12)$, showing the highest whole blood levels (lowest Ct values) in leukocyte-associated microRNAs-21, -29b, -126 and -223. Panel (B) displays the difference between plasma and whole blood Ct per patient. The liver-specific miR-122 is not detected earlier in whole blood, as expected due to its exclusive liver specificity. Interestingly, leukocyte-associated microRNAs show highly similar patterns in plasma and whole blood (all were detected $\sim 11$ cycles earlier in whole blood than plasma regardless of expression level), indicating that these plasma microRNA are predominantly derived from leukocytes. Since plasma microRNAs did not correlate with leukocyte counts at the individual patient level (Supplementary Figure 5), these findings may suggest that variable leakage of microRNAs from leukocytes distorts the diagnostic accuracy of plasma microRNAs. Error bars represent standard deviations. 
Supplementary Table 1. Acute Myocardial Infarction Database Patient Characteristics

\begin{tabular}{lccc}
\hline & $\begin{array}{c}\text { Acute Myocardial Infarction } \\
\text { Characteristic }\end{array}$ & Controls \\
(n=36) & (n=36) & P* \\
\hline Age, years & $62 \pm 13$ & $62 \pm 13$ & 0.95 \\
Women & $12(33.3)$ & $13(36.1)$ & 0.86 \\
Sample age, months & $80 \pm 10$ & $94 \pm 1$ & $<0.0001$ \\
Serum Troponin T, $\mu \mathrm{g} / \mathrm{L}$ & $3.5 \pm 4.8$ & $<0.01$ & $<0.0001$ \\
NT-proBNP, pmol/L & $615 \pm 678$ & $366 \pm 835$ & 0.36 \\
Serum hs-CRP, mg/L & $0.74 \pm 0.80$ & $0.46 \pm 0.79$ & 0.23 \\
PTCA, n & $36(100)$ & $1(2.8)$ & $<0.0001$ \\
\hline
\end{tabular}

Data are presented as mean $\pm \mathrm{SD}$, or $n(\%),{ }^{*}$ P-values based on student t-test or Chi-square analysis, CPK Creatine Phosphokinase NT-proBNP, hs-CRP High-Sensitivity C-Reactive Protein, N-terminal Prohormone Brain Natriuretic Peptide, PTCA Percutaneous Transluminal Coronary Angioplasty

Supplementary Table 2. Viral Myocarditis Database Patient Characteristics

\begin{tabular}{lcccc}
\hline Characteristic & $\begin{array}{c}\text { Acute VM } \\
(\mathbf{n = 1 4 )}\end{array}$ & $\begin{array}{c}\text { Post VM } \\
(\mathbf{n = 2 0 )}\end{array}$ & $\begin{array}{c}\text { Controls } \\
(\mathbf{n = 2 0 )}\end{array}$ & $\mathbf{P}$ \\
\hline Age, years & $32.7 \pm 16.3$ & $37.3 \pm 19.4$ & $32.1 \pm 7.3$ & $0.51^{*}$ \\
Women & $2(14.2)$ & $3(15)$ & $8(40)$ & $0.96 \dagger$ \\
Sample age, months & $19 \pm 13$ & $22 \pm 6$ & $6 \pm 0.4$ & $<0.001^{*}$ \\
Serum CRP, mg/L & $95 \pm 92$ & $11 \pm 14$ & $\mathrm{ND}$ & $<0.005 \ddagger$ \\
Serum troponin T, $\mu \mathrm{g} / \mathrm{L}$ & $1.1 \pm 1.6$ & $<0.01$ & $\mathrm{ND}$ & $<0.05 \neq$ \\
Blood leukocytes, $\times 10 \% / \mathrm{L}$ & $12.5 \pm 5.5$ & $7.1 \pm 3.1$ & $\mathrm{ND}$ & $<0.005 \neq$ \\
LVEF, \% & $48.3 \pm 14.1$ & $57.2 \pm 7.6$ & $\mathrm{ND}$ & $<0.001^{*}$ \\
\hline
\end{tabular}

Data are presented as mean $\pm \mathrm{SD}$, or $\mathrm{n}(\%)$, P-values based on * One-way ANOVA, † Chi-square analysis or $¥$ Student's t-test or Mann-Whitney test, CRP C-Reactive Protein, LVEF Left Ventricular Ejection Fraction, ND Not determined 
Supplementary Table 3. Diastolic Dysfunction Patient Baseline Characteristics

\begin{tabular}{|c|c|c|c|c|}
\hline Characteristic & $\begin{array}{l}\text { Controls } \\
(n=20)\end{array}$ & $\begin{array}{l}\text { Hypertension } \\
\qquad(\mathrm{n}=20)\end{array}$ & $\begin{array}{c}\text { Diastolic } \\
\text { Dysfunction } \\
\text { (n=39) }\end{array}$ & $\mathbf{P}^{*}$ \\
\hline Age, years & $63 \pm 7$ & $60 \pm 8$ & $69 \pm 8$ & $<0.001$ \\
\hline Women & $9(45)$ & $8(40)$ & $17(44)$ & 0.95 \\
\hline Sample age, months & $33 \pm 11$ & $33 \pm 11$ & $37 \pm 11$ & 0.31 \\
\hline $\mathrm{BMI}, \mathrm{kg} / \mathrm{m}^{2}$ & $24.8 \pm 2.3$ & $27.3 \pm 4.3$ & $28.8 \pm 3.4$ & $<0.001$ \\
\hline $\mathrm{SBP}, \mathrm{mm} \mathrm{Hg}$ & $121 \pm 8$ & $157 \pm 15$ & $150 \pm 13$ & $<0.0001$ \\
\hline Creatinine, $\mu \mathrm{mol} / \mathrm{L}$ & $86 \pm 17$ & $90 \pm 14$ & $90 \pm 16$ & 0.86 \\
\hline GFRt, $\mathrm{mL} / \mathrm{min}$ & $73 \pm 17$ & $80 \pm 16$ & $72 \pm 21$ & 0.29 \\
\hline$\nu \mathrm{GT}$, units/L & $31 \pm 20$ & $36 \pm 21$ & $32 \pm 25$ & 0.78 \\
\hline WBC count, $\times 10^{9} / \mathrm{L}$ & $6.1 \pm 2.1$ & $5.6 \pm 1.4$ & $5.7 \pm 1.7$ & 0.66 \\
\hline Glucose, mmol/L & $4.9 \pm 0.3$ & $5.1 \pm 0.8$ & $5.2 \pm 1.7$ & 0.55 \\
\hline NT-proBNP, pmol/L & $245 \pm 118$ & $268 \pm 155$ & $337 \pm 159$ & 0.09 \\
\hline LVMI, g/m² & $88 \pm 12$ & $100 \pm 18$ & $117 \pm 22$ & $<0.0001$ \\
\hline Current smoker & $6(30)$ & $5(25)$ & $4(10)$ & 0.14 \\
\hline
\end{tabular}

Data are presented as mean $\pm S D$, or $n(\%), *$ P-values based on One-way ANOVA or Chi-square analysis, $\dagger$ Glomerular filtration rates were calculated using the Cockroft-Gault formula, BMI Body Mass Index, EDP End Diastolic Pressure, GFR Glomerular Filtration Rate, LVMI Left Ventricular Mass Index, NT-proBNP N-terminal Prohormone Brain Natriuretic Peptide, SBP Systolic Blood Pressure

Supplementary Table 4. Acute Heart Failure Database Patient Characteristics

\begin{tabular}{lccc}
\hline & $\begin{array}{c}\text { Acute Decompensation } \\
\text { Characteristic }\end{array}$ & $\begin{array}{c}\text { Healthy Controls } \\
(\mathbf{n = 3 3})\end{array}$ & $\mathbf{P}^{*}$ \\
\hline Age, years & $76.3 \pm 8.4$ & $34.8 \pm 17.1$ & $<0.0001$ \\
Women & $20(60.1)$ & $16(59.6)$ & 0.82 \\
Sample age, months & $6.5 \pm 2.4$ & $6.9 \pm 2.0$ & 0.50 \\
NT-proBNP, pmol/L & $1590 \pm 2022$ & $\mathrm{ND}$ & \\
LVEF, \% & $37.0 \pm 15.0$ & $\mathrm{ND}$ & \\
Preserved LVEF $>$ 45\% & $15(45)$ & & \\
Underlying pathology & & \\
CAD & $19(57.5)$ & \\
Valvular & $6(18.2)$ & \\
\hline
\end{tabular}

Data are presented as mean $\pm \mathrm{SD}$, or $\mathrm{n}(\%),{ }^{*}$ P-values based on student t-test or Chi-square analysis, CAD Coronary Artery Disease, CRP C-Reactive Protein, LVEF Left Ventricular Ejection Fraction, ND Not determined, NT-proBNP N-terminal Prohormone Brain Natriuretic Peptide 





Supplementary Table 6a. MicroRNA level intercorrelations in Diastolic Dysfunction Plasmas (correlation coefficient R)

\begin{tabular}{|c|c|c|c|c|c|c|c|c|}
\hline$R$ values & miR-21 & miR-29b & miR-126 & miR-146a & miR-146b & miR-223 & miR-133a* & miR-208b* \\
\hline miR-21 & $x$ & & & & & & & \\
\hline miR-29b & $>0.75$ & $x$ & & & & & & \\
\hline miR-126 & $>0.75$ & $>0.75$ & $x$ & & & & & \\
\hline miR-146a & $>0.75$ & $>0.75$ & $>0.75$ & $x$ & & & & \\
\hline miR-146b & $>0.75$ & $>0.75$ & $>0.75$ & $>0.75$ & $x$ & & & \\
\hline miR-223 & $>0.75$ & $>0.75$ & $>0.75$ & $>0.75$ & $>0.75$ & $x$ & & \\
\hline miR-133a* & $<0.2$ & $<0.2$ & $<0.2$ & $<0.2$ & $<0.2$ & $<0.2$ & $x$ & \\
\hline miR-208b* & $<0.2$ & $<0.2$ & $<0.2$ & $<0.2$ & $<0.2$ & $<0.2$ & $<0.2$ & $x$ \\
\hline
\end{tabular}

* Non-leukocyte associated microRNAs

Supplementary Table 6b. MicroRNA level inter-correlations in Diastolic Dysfunction Plasmas (P values)

\begin{tabular}{|c|c|c|c|c|c|c|c|c|}
\hline P-value & miR-21 & miR-29b & miR-126 & miR-146a & miR-146b & miR-223 & miR-133a* & miR-208b* \\
\hline miR-21 & $x$ & & & & & & & \\
\hline miR-29b & $<0.0001$ & $x$ & & & & & & \\
\hline miR-126 & $<0.0001$ & $<0.0001$ & $x$ & & & & & \\
\hline miR-146a & $<0.0001$ & $<0.0001$ & $<0.0001$ & $x$ & & & & \\
\hline miR-146b & $<0.0001$ & $<0.0001$ & $<0.0001$ & $<0.0001$ & $x$ & & & \\
\hline miR-223 & $<0.0001$ & $<0.0001$ & $<0.0001$ & $<0.0001$ & $<0.0001$ & $x$ & & \\
\hline miR-133a* & 0.24 & 0.69 & 0.11 & 0.17 & 0.34 & 0.30 & $x$ & \\
\hline miR-208b* & 0.16 & 0.54 & 0.55 & 0.39 & 0.89 & 0.97 & 0.42 & $x$ \\
\hline
\end{tabular}

* Non-leukocyte associated microRNAs 

General Discussion 
MicroRNAs (miRNAs) are important regulators of pre-mammalian and mammalian gene expression and their involvement in cardiovascular disease has been well documented ${ }^{1-3}$. Most studies have focused on the role of miRNAs in processes such as cardiomyocyte hypertrophy, angiogenesis, apoptosis and fibrosis ${ }^{4-7}$. In contrast, miRNA regulation of cardiac inflammation, which is critically involved in the pathogenesis of heart failure, has hitherto not been addressed ${ }^{8,9}$. Therefore, the first aim of this thesis was to study miRNA signaling in cardiac inflammation during different forms of heart failure. We approached this question by (1) screening for cardiac miRNA expression alterations during acute viral myocarditis and next by (2) addressing the cardiac response to pressure overload in mice deficient for the pro-inflammatory microRNA-155 (miR-155). The main conclusions from these studies are that miRNAs are important contributors to inflammatory signaling and heart failure development in both myocarditis and hypertensive heart failure, and that their adverse effects can be therapeutically targeted by pharmacological inhibition in rodents. During viral myocarditis, many miRNAs are dysregulated in the hearts of human patients and mice (Chapter 4). Among them, miR-155 is consistently and strongly upregulated during the acute inflammatory phase. Pharmacological inhibition of miR-155 reduces cardiac injury in the acute phase and improves survival (Chapter 4). In contrast, signaling by the miR-221miR-222 family is protective against viral replication, cardiac injury, and inflammatory infiltration during acute viral myocarditis (Chapter 5). Following pressure overload, miR-155 controls the activation and cardiac infiltration of immune cells. Absence or inhibition of miR-155 attenuates pro-hypertrophic signaling by cardiac macrophages and diminishes cardiac hypertrophy as well as systolic dysfunction (Chapter 6).

The second aim of this thesis was to explore the potential of using miRNAs as biomarkers for cardiovascular diseases in patients. By studying levels of individual miRNAs in plasma samples from patients suffering from acute myocardial infarction, viral myocarditis, heart failure, and from controls, we discovered that the cardiac-enriched miRNAs miR-208b and miR-499 are sensitive indicators of myocardial damage (Chapter 7). Levels of these miRNAs are strongly elevated in patients with acute myocardial infarction and viral myocarditis, and correlate tightly with biochemical markers of cardiomyocytes death, such as cardiac troponin T. These alterations were independent of clinical characteristics such as age, gender, body mass index, and renal function, and may therefore be clinically applicable in the diagnosis of acute myocardial infarction.

\section{MicroRNAs as mediators of viral myocarditis}

Viral infection is the most prevalent cause of acute myocarditis in Western society and the treatment of virus-induced myocarditis represents a difficult clinical problem ${ }^{30}$. Since both the virus and the immune response against it contribute to cardiac injury and the development of dilated cardiomyopathy, broad inhibition of inflammation does not lead to patient benefit as it does in non-viral myocarditis ${ }^{31-34}$. Over the past decade, accumulating insights into the beneficial versus detrimental nature of specific immune response components (cellular or molecular) suggest that targeted treatment of viral myocarditis 
may be feasible, as reviewed in Chapter 3. For instance, toll-like receptor-3 signaling through TRIF is necessary for efficient antiviral defence and cardiac protection, while TLR-4 and TLR-9 signaling through the molecular adapter MyD88 promote cardiac injury and mortality ${ }^{35-37}$. Similarly, endogenous inhibitors of inflammation, such as alternatively activated macrophages or regulatory T cells, attenuate viral replication as well as myocarditis in mice ${ }^{38,39}$. While such findings establish a clear rationale for targeted treatment, development and testing of specific inhibitors is a challenging and time-consuming endeavour.

MiRNAs play important roles in numerous diseases and can efficiently be targeted in vivo in mice and primates through a variety of chemistries ${ }^{40-42}$. They are attractive therapeutic targets due to the straightforward design of antimiRs - based on Watson-Crick complementarity - and the generally subtle effects of miRNAs on signaling networks ${ }^{2,43,}$ 44. To map the behaviour of miRNAs during viral myocarditis, we have longitudinally profiled miRNA alterations in the hearts of human patients as well as myocarditis-susceptible and -resistant mouse strains (Chapter 4). A large number of miRNAs is up or downregulated during acute myocarditis, including miR-155 that is elevated in the hearts of both mice and humans during myocarditis. Inhibition of miR-155 in mice suppresses T cell activation and cardiac infiltration by myeloid cells, which reduces cardiac necrosis in the acute phase and mortality in the long run (Chapter 4 and Figure 1). Previous studies have demonstrated that miR-155 suppression ameliorates autoimmune diseases, such as experimental autoimmune encephalitis and rheumatoid arthritis ${ }^{45,46}$. The therapeutic benefit of miR-155 suppression in a combined infectious-autoimmune disease such as viral myocarditis complements these observations and shows that miR-155 may be a molecularly targetable switch to improve outcome in viral myocarditis patients. In contrast to miR-155, inhibition of the miR-221-222 family severely aggravates cardiac inflammation and injury in mice, at least in part by promoting viral replication in the heart (Chapter 5 and Figure 1). Since cardiac miR-221 and miR-222 are upregulated in mouse myocarditis, but downregulated in cardiac biopsies of humans during viral myocarditis, restoring their expression levels in the hearts of human patients may be of therapeutic benefit.

In addition to miRNA dysregulation during acute myocarditis, we studied the different response in early miRNA signaling between myocarditis susceptible $(\mathrm{C} 3 \mathrm{H})$ and resistant (C57Bl/6) mice. Intriguingly, profiles of dysregulated miRNAs at day 4 after infection with the cardiotropic Coxsackievirus B3 were markedly divergent between these strains, despite equal viral replication in the heart. These differences may be responsible for the differential generation of immune responses and provide clues about the determinants of myocarditis susceptibility between humans.

While our study is the first do describe the expression and functional roles of miRNAs during viral myocarditis, we have likely uncovered the mere tip of the iceberg and the study generates several important questions. During what phases of disease is modulation of miRNAs such as miR-155 and miR-221/miR-222 effective and how long should treatment continue? Which miRNAs are involved in the progression of acute into chronic myocarditis? Can miRNA function during enteroviral myocarditis be extrapolated to other increasingly prevalent viral causes of myocarditis, such as parvovirus B19 and human herpes virus 6 ? 
Systematic investigation of the last question requires analysis of endomyocardial biopsies (EMB). EMB is infrequently performed in patients with acute viral myocarditis since many patients with acute myocarditis spontaneously recover. Unfortunately, this complicates the association of therapeutic outcomes with molecular disease markers (viral, miRNA) that is needed to develop more targeted treatments for this heterogeneous group of patients. In addition, non-standardized EMB performance across institutions fosters variation between clinical studies. Recent discoveries provide arguments in favor of more frequent EMB performance. First, specific treatments benefit EMB-identifiable patient subgroups, such as immunosuppression and interferon-beta administration in non-viral and viral myocarditis, respectively ${ }^{33,48}$. Secondly, the establishment of non-invasive prognostic parameters during the acute phase allows to restrict EMB sampling to patients at risk for poor outcome, such as those with late gadolinium enhancement on cardiac magnetic resonance imaging ${ }^{49}$. These findings help pave the way towards integration of lessons from cardiac miRNA biology into patient care.

\section{Viral myocarditis}

Pressure overload

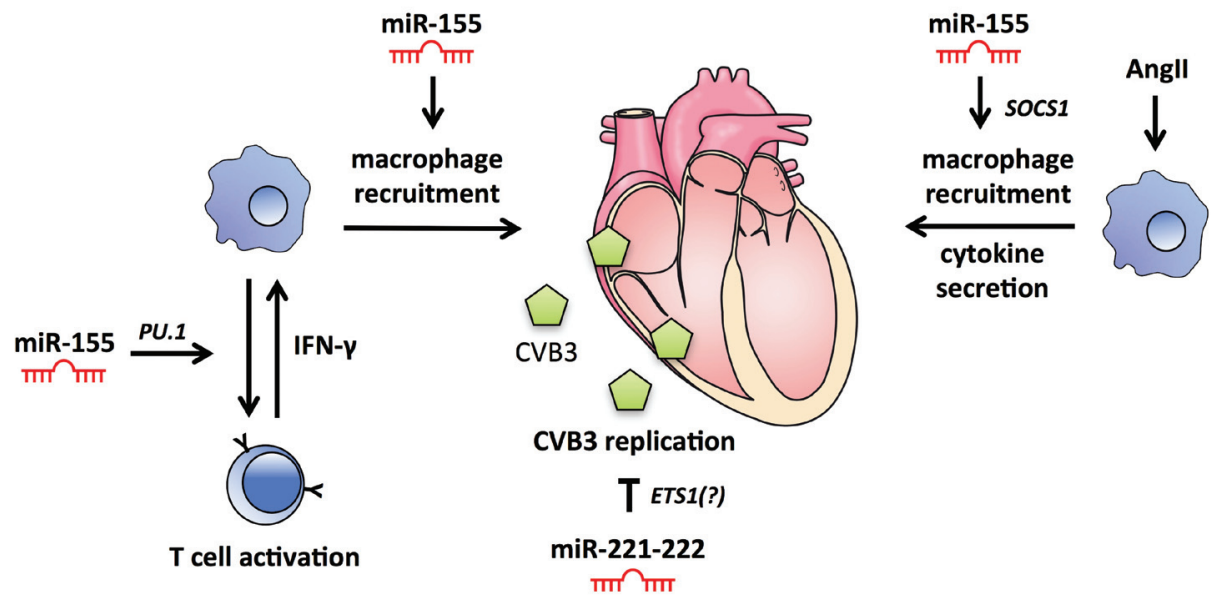

Figure 1. Effects of microRNA-155 (miR-155) and miR-221-222 in the heart during cardiac inflammation. Inflammatory injury to the heart during viral myocarditis is mediated by the pro-inflammatory effects of miR155. MiR-155 is expressed by immune cells, including macrophages and T lymphocytes, and stimulates T cell activation by targeting genes involved in the regulation of immune responses, such as PU.1. In addition, miR-155 promotes macrophage recruitment to the heart, thereby augmenting the local inflammatory activity. The miR-221-222 family inhibits replication of Coxsackievirus B3 (CVB3) in cardiomyocytes and protects against virus accumulation and cardiac inflammation. This effect may be mediated by the direct miR-221222 target ETS1, that is known to promote the replication RNA viruses. In addition to viral myocarditis, inflammation is also a hallmark of pressure overload-induced cardiac disease, which is mediated partly by activation of the pro-inflammatory vasoconstrictor hormone Angiotensin II (AnglI). During pressure-induced cardiac inflammation, miR-155 promotes the recruitment of monocyte-macrophages into the blood, their infiltration into the heart, and stimulates their secretion of pro-inflammatory and pro-hypertrophic cytokines through targeting the inhibitor of cytokine secretion SOCS1. 


\section{Inflammation and miR-155 in the pressure-overloaded heart}

Chronic arterial hypertension increases cardiac workload and ventricular wall stress. The human heart accommodates for such changes by adapting its shape and function in a process called hypertrophic remodeling. In this process, cardiac enlargement is associated with increased size and contractile organization of individual myocytes ${ }^{10}$. In the short run this is an adaptive, compensatory response that results in wall stress normalization. However, in the long run, cardiac hypertrophy is associated with progression to heart failure, arrhythmias, and sudden death, independent of the underlying trigger ${ }^{10,11}$. In line with these observations, inhibition or reversal of hypertrophy in patients by treatment with angiotensinconverting enzyme inhibitors lowers their risk for death and heart failure progression, while persistent hypertrophy predicts adverse outcomes independent from blood pressure responses ${ }^{12}$. Hypertrophic remodeling is therefore considered a therapeutic target in cardiovascular medicine. The molecular mechanism of myocyte hypertrophy is complex and involves activation of hypertrophic gene programs through biomechanical stress or extracellular triggers including neurohormones (angiotensin II, endothelin-1, catecholamines) and peptide growth factors (such as epidermal growth factor, fibroblast growth factor-2 (FGF2), insulin-like growth factor- 1$)^{13}$. Over the past decade it has become well accepted that signaling by adjacent cardiac cells, such as fibroblasts and endothelial cells, is of critical importance for hypertrophy. For instance, fibroblasts stimulate myocyte hypertrophy by secreting pro-hypertrophic factors including transforming growth factor-beta (TGF- $\beta$ ), FGF2 and members of the interleukin 6 (II-6) family ${ }^{14}$.

In line with the pro-hypertrophic effects of the inflammatory cytokine II-6, the immune system is also emerging as a contributor to hypertrophic signaling, as supported by a number of observations. First, pressure overload causes cardiac infiltration of activated immune cells, which precedes hypertrophic remodeling (Chapter 4). Second, myocyte hypertrophy can be triggered by multiple cytokines secreted from infiltrated immune cells, including II-6, I-1 $\beta$, tumor necrosis factor, and TGF- $\beta^{10,13,15-17}$. Third, several pro-hypertrophic signal transduction pathways are also essential pro-inflammatory pathways in immune cells, such as calcineurin-nuclear factor of activated T cells (NFAT), nuclear factor kappa B (NF-kB), signal transducer and activator of transcription 3 (STAT3), and phosphoinositide 3 kinase gamma (PI3K) signaling ${ }^{18-20}$. Finally, infusion with regulatory $T$ cells (a class of $T$ cells with broad anti-inflammatory effects) blunts cardiac hypertrophy during angiotensin-induced pressure overload in mice independent from blood pressure changes ${ }^{21}$. Beyond promoting hypertrophy, inflammatory cytokines also directly impact the progression of heart failure by inducing myocyte death, fetal gene expression, contractile dysfunction, and interstitial fibrosis leading to left ventricular dysfunction, pulmonary edema, and cardiomyopathy ${ }^{22}$. In patients, circulating levels of tumor necrosis factor (TNF- $\alpha$ ), interleukin-1 $\beta$ (II-1 $\beta$ ) and II-6 are elevated earlier during heart failure progression than are classical neurohormones ${ }^{23}$ and TNF- $\alpha$ and II-6 levels are predictors of mortality independent of age, left ventricular ejection fraction and New York Heart Association (NYHA) class ${ }^{24}$. In conclusion, a firm rationale supports the investigation of inflammation in hypertrophic remodeling and heart failure, 
and of the miRNAs controlling inflammatory activity as potential therapeutic targets for these diseases ${ }^{8}$.

MiRNAs are expressed by many cell types in the heart, as evidenced by a large number of miRNAs whose cardiac levels are not affected by myocyte-restricted Dicer deletion in the post-natal mouse heart ${ }^{25}$. We found that among these miRNAs, miR-155 is expressed by infiltrating inflammatory cells in the pressure-overloaded heart and contributes to prohypertrophic paracrine signaling from macrophages to cardiomyocytes (Chapter 6 and Figure 1). MiR-155 promotes cardiac inflammation in response to angiotensin II infusion at multiple levels, as miR-155 deficiency results in decreased monocyte recruitment to circulating blood, reduces numbers of macrophages infiltrating the heart, and attenuates pro-inflammatory cytokine secretion from infiltrating cells. Attenuated inflammation dampened cardiac hypertrophy and consequent systolic dysfunction in mice without altering blood pressure responses to angiotensin II. These effects were primarily leukocyteautonomous and based on miR-155-dependent pro-hypertrophic effects of the macrophage secretome on myocytes (Chapter 6). These data add new weight to the importance of the cardio-inflammatory crosstalk and suggest that targeting miR-155 may be of benefit in the treatment of hypertrophic remodeling.

Our work raises a number of general questions. For instance, what paracrine factors from macrophages promote myocyte hypertrophy? While cytokines such as TNF- $\alpha$ and the II-1 and II-6 family may fully account for hypertrophic stimulation, other secreted factors may also be involved. We have found a large number of protein levels altered in the secretomes of miR-155 wild-type versus knockout macrophages. An interesting approach would be to systematically study the hypertrophic effects of secretome fractions defined by size or sensitivity to proteolytic versus ribonucleic digestion. Another question coined is whether the observed protection of contractile function is secondary to blunted hypertrophy or caused by a parallel mechanism such as an attenuated negative-inotropic cytokine milieu. Also, might inflammation be the trigger that differentiates between physiological and pathological hypertrophy? While the lack of inflammation associated with physiological hypertrophy in athletes supports such a hypothesis, NF-kB is necessary for adaptive physiological hypertrophy in mice ${ }^{26}$. In contrast, inhibition of miR-155 leads to decreased hypertrophy and preserves cardiac function (Chapter 6). Therefore, it may be the nature and severity, rather than the occurrence of inflammation that determines outcome. It is noteworthy that such dose-dependent phenotypic outcomes of signaling are recurrently observed in inflammatory and cardiovascular signaling. Inflammatory responses protect the organism against acute threats while chronic inflammatory activation is the cause of many human diseases. In parallel, initially compensatory hypertrophy is detrimental in the setting of chronic overload. Also, the hypertrophic and inflammatory signaling pathways are often myocyte pro-survival pathways in acute settings (such as transient or permanent myocardial ischemia) while chronic activation is deleterious ${ }^{27-29}$. Remedying chronic effects of such pathways will become more urgent due to the increasing chronic disease burden in our progressively aging Western population. 


\section{Plasma miRNAs as biomarkers of cardiovascular disease}

The stability and detectability of miRNAs released into the circulation offers opportunities for their use as biomarkers for human disease. By comparing plasma miRNA levels in patients suffering from cardiovascular disease - including acute myocardial infarction (AMI), viral myocarditis (VM), heart failure and diastolic dysfunction - we identified miR-208b and miR-499 as sensitive indicators of cardiomyocyte injury (Chapter 7 and Figure 2). Both miRNAs are strongly enriched in muscular cells and their plasma levels in the general population are low to absent. Their plasma levels are not affected by typical clinical confounding parameters (age, gender, arterial blood pressure, body-mass index, renal function) but rise sharply during acute myocardial infarction and viral myocarditis. Elevations correlated strongly with circulating levels of troponin $T$ and could accurately discriminate AMI patients from controls (Chapter 7). Plasma levels of two other myocyte-enriched miRNAs, miR-1 and miR-133b, were also elevated in AMI patients, albeit less profoundly. In patients with diastolic dysfunction, a condition not associated with myocyte death, we found no significant differences in any of the miRNAs studied.

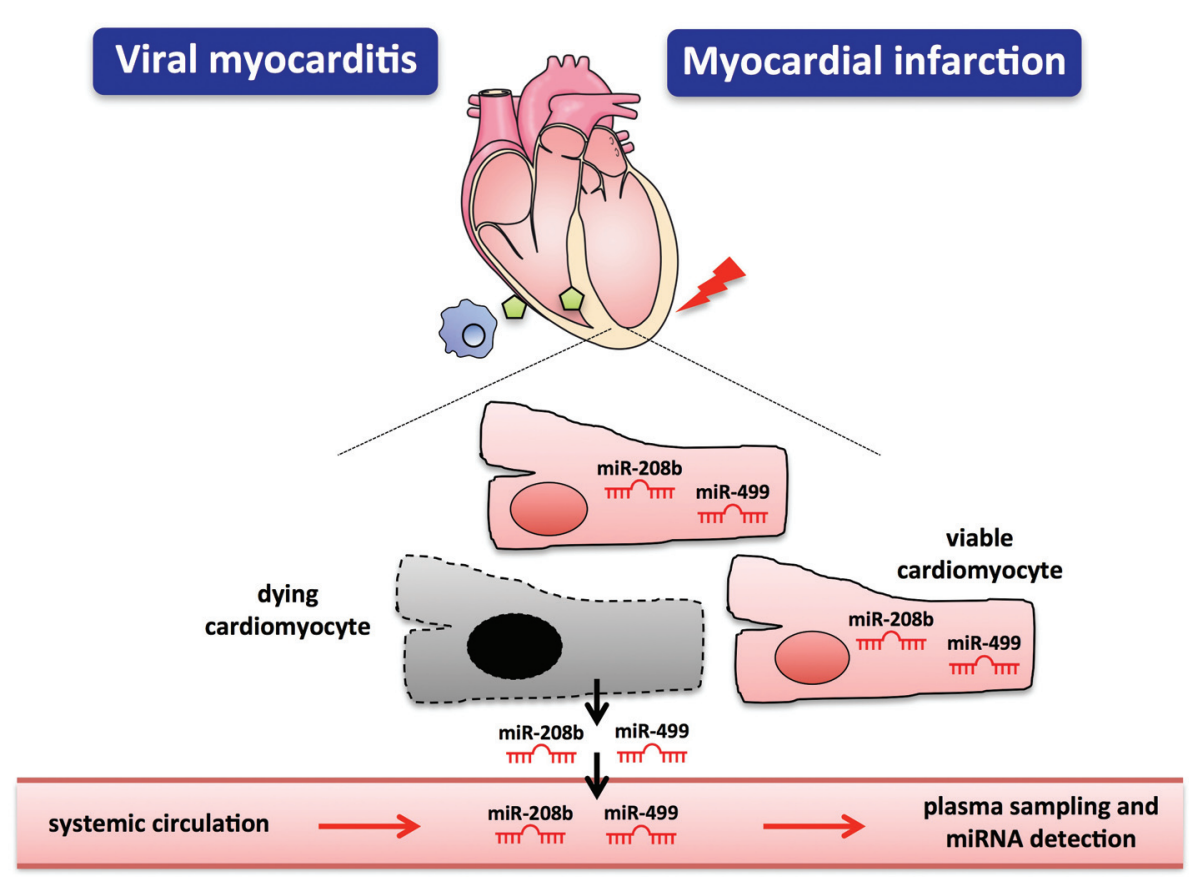

Figure 2. Cardiomyocyte damage leads to the release of myocyte-enriched miRNAs into the systemic circulation. MiR-208b and miR-499 are highly expressed in viable cardiomyocytes and are hardly detectable in plasma samples of healthy individuals. However, during acute cardiac damage caused by acute myocardial infarction or viral myocarditis, dying myocytes release miR-208b and miR-499 into the blood stream. The resulting elevation of miR-208b and miR-499 can be detected in plasma samples, allowing their use as early and sensitive biomarkers of cardiac damage. 
Finally, we hypothesized that assessment of inflammatory miRNAs in addition to miR-208b and miR-499 might aid the clinically challenging differential diagnosis between $\mathrm{AMI}$ and VM. However, levels of inflammation-associated miR-146a, miR-146b, miR-155 and miR223 were not significantly elevated or depressed during VM. Though miR-223 levels were mildly lower in AMI patients versus controls, we did not find evidence supporting a strong discriminatory potential of inflammation-associated miRNAs. Rather, plasma patterns of miRNAs enriched in circulating blood cells strongly resembled the miRNA patterns in these cells, suggesting that leakage of such miRNA from blood cells confounds their plasma levels and diagnostic potential (Chapter 7). These findings have been corroborated by other groups ${ }^{50,51}$, indicating that miRNAs not enriched in blood cells, such as the myocyteenriched miRNAs may be most suited as biomarkers for cardiovascular disease.

Our findings with regard to myocyte-miRNA elevation in AMI patient plasma have been confirmed by a number of independent studies during the preparation and after publication of our manuscript ${ }^{52-56}$. To be clinically useful, biomarkers must provide information not available from clinical assessment or existing blood tests ${ }^{57}$. For AMI diagnosis, the most widely used tests measure plasma levels of cardiac troponins. The major limitation of these tests is their low sensitivity at the time of patient presentation due to a delayed increase in circulating levels of cardiac troponins. This necessitates serial blood testing up to 6 hours after onset of complaints and causes treatment delay and increased complications ${ }^{58}$. While the recently introduced high-sensitivity troponins (hs-TNT and hs-TNI) have markedly improved early diagnostic accuracy, there is considerable room left for improvement since the negative predictive value of hs-TNI testing within 3 hours after onset of complaints does not exceed $84 \%{ }^{59,60}$. We have therefore expanded upon the findings presented in Chapter 6 and studied the diagnostic performance of miR-208b and miR-499 in comparison to hs-TNT in a larger cohort of AMI patients $(n=510)$ with a focus on early diagnosis. MiRNA and hs-TNT levels were both detectable in plasma from 1 hour after onset of complaints and 3 hours after onset the percentage miR-499 positivity was by trend higher than hs-TNT positivity $(93 \% \text { versus } 88 \%)^{61}$. However, both miRNA and hs-TNT testing provided comparably high diagnostic value (areas under the curve of 0.97) and miR-499 levels did not significantly contribute to a clinical classification model including hs-TNT as well as several risk factors ${ }^{61}$. In agreement, a study investigating miRNA levels in plasma of $n=444$ patients with acute coronary syndromes reported that miR-133a and miR-208b were associated with risk of death after adjusting for age and gender but lost their association after adjusting for hs-TNT ${ }^{56}$. While these findings suggest that miRNAs are powerful but not superior biomarkers for $\mathrm{AMI}$ in comparison to high-sensitivity troponins, a number of practical considerations must be taken into account before definite conclusions can be drawn. First, technical differences in isolation and miRNA measurements between labs considerably impact elementary outcomes, such as the measurability of low abundance miRNAs in controls and the reported fold-increases of miRNAs ${ }^{62,63}$. Improved and standardized measurements will be instrumental in integrating study results for generalizable conclusions. In addition, both detectability and kinetics of miRNA increases after onset of complaints affect diagnostic usefulness: while miR-499 performed as good as hs-TNT in 
our hands at 3 hours after onset of complaints, the miR-499 levels peak several hours later than those of miR-208, miR-1 and miR-13353,54. Finally, the straightforward measurement of different miRNAs facilitates the integration of multiple miRNAs into a multiplex classifier that may outperform single biomarkers ${ }^{64}$.

In conclusion, we and others have demonstrated the feasibility of using miRNAs as plasma biomarkers for cardiovascular disease. Improvements in isolation techniques are required to adequately address their potential for clinical translation. From a general perspective, these findings have raised vexing questions regarding the significance of cellular export or leakage of miRNAs and their potential biological functions. Though myocyte necrosis is likely an important source of myocyte miRNA elevation in plasma, miR-499 levels are downregulated in infarcted hearts ${ }^{54}$, possibly indicating active cellular miRNA export. Similarly, liver miRNAs elevated in plasma during drug-induced hepatotoxicity are simultaneously downregulated in hepatic tissue ${ }^{65}$. In addition, proteins and lipid carriers carry distinct miRNA populations in plasma and the cellular export of these populations is differently regulated ${ }^{6-69}$. Selective measurements of miRNAs in these compartments may therefore provide novel dimensions of diagnostic information. Finally, some lines of evidence suggest that plasma miRNAs may be involved in paracrine signaling after uptake in remote cells ${ }^{67,70-73}$. Efforts are ongoing to better understand the basic biology underlying the phenomenon of plasma miRNA, and these insights may translate into clinical applications within the next decade.

\section{Conclusions and future perspectives}

This thesis presents important roles for miRNAs during inflammation in hypertrophic and inflammatory heart disease, as well as their use as biomarkers for cardiac disease. The beneficial effect of suppressing cardiac inflammation through miR-155 has two important implications. First, it emphasizes the importance of inflammation as a contributor and therapeutic target in heart failure. Secondly, it directly suggests that miR-155 inhibitors may benefit patients with viral myocarditis or myocardial hypertrophy. The clinical translation of these findings will nevertheless require rigorous additional studies. Though miRNA inhibitors have been successfully tested in non-human primates and are currently in phase II clinical trials ${ }^{41,42,74}$, an attractive feature of miRNA therapeutics also represents a disadvantage: the nuanced effects on gene expression through concerted repression of hundreds of mRNA targets complicate predictions of side-effects. From a mechanistic perspective, the multitude of miRNA targets also complicates pinpointing single targets as responsible for miRNA loss-of-function phenotypes. MiRNA biology challenges the traditional approach to biological interactions as more or less linear signaling pathways by adding a new dimension of complexity. This complexity involves dynamic regulation of extensive signaling networks with complex feedback and feed-forward systems. Systems biology approaches will be indispensable for studying these signaling environments, and a key challenge for molecular biologists will be to translate the generated complexity into 
understandable knowledge and intuitive heuristics. In contrast, utilizing miRNAs as biomarkers for cardiovascular disease does not require in-depth understanding of their biological roles but will require standardized methods for reproducible measurements across different centers. In spite of these challenges, our knowledge about the role of miRNAs in cardiovascular disease accumulates rapidly and their translation into clinically applicable therapeutic and diagnostic tools is probably a matter of time. 


\section{References}

1. Bartel DP. Micrornas: Target recognition and regulatory functions. Cell. 2009;136:215-233

2. Small EM, Olson EN. Pervasive roles of micrornas in cardiovascular biology. Nature. 2011;469:336-342

3. Schroen B, Heymans S. Micrornas and beyond: The heart reveals its treasures. Hypertension. 2009;54:1189-1194

4. van Rooij E, Sutherland LB, Liu N, Williams AH, McAnally J, Gerard RD, Richardson JA, Olson EN. A signature pattern of stress-responsive micrornas that can evoke cardiac hypertrophy and heart failure. Proc Natl Acad Sci U S A. 2006;103:18255-18260

5. Thum T, Gross C, Fiedler J, Fischer T, Kissler S, Bussen M, Galuppo P, Just S, Rottbauer W, Frantz S, Castoldi M, Soutschek J, Koteliansky V, Rosenwald A, Basson MA, Licht JD, Pena JT, Rouhanifard SH, Muckenthaler MU, Tuschl T, Martin GR, Bauersachs J, Engelhardt S. Microrna-21 contributes to myocardial disease by stimulating map kinase signalling in fibroblasts. Nature. 2008;456:980-984

6. da Costa Martins PA, Salic K, Gladka MM, Armand AS, Leptidis S, el Azzouzi H, Hansen A, Coenen-de Roo CJ, Bierhuizen MF, van der Nagel R, van Kuik J, de Weger R, de Bruin A, Condorelli G, Arbones ML, Eschenhagen T, De Windt LJ. Microrna-199b targets the nuclear kinase dyrk1a in an auto-amplification loop promoting calcineurin/nfat signalling. Nature cell biology. 2010;12:1220-1227

7. Bonauer A, Carmona G, Iwasaki M, Mione M, Koyanagi M, Fischer A, Burchfield J, Fox H, Doebele C, Ohtani K, Chavakis E, Potente M, Tjwa M, Urbich C, Zeiher AM, Dimmeler S. Microrna-92a controls angiogenesis and functional recovery of ischemic tissues in mice. Science. 2009;324:1710-1713

8. Heymans S, Hirsch E, Anker SD, Aukrust P, Balligand JL, Cohen-Tervaert JW, Drexler H, Filippatos G, Felix SB, Gullestad L, Hilfiker-Kleiner D, Janssens S, Latini R, Neubauer G, Paulus WJ, Pieske B, Ponikowski P, Schroen B, Schultheiss HP, Tschope C, Van Bilsen M, Zannad F, McMurray J, Shah AM. Inflammation as a therapeutic target in heart failure? A scientific statement from the translational research committee of the heart failure association of the european society of cardiology. Eur J Heart Fail. 2009;11:119-129

9. Marchant DJ, Boyd JH, Lin DC, Granville DJ, Garmaroudi FS, McManus BM. Inflammation in myocardial diseases. Circulation research. 2012;110:126-144

10. Frey N, Olson EN. Cardiac hypertrophy: The good, the bad, and the ugly. Annual review of physiology. 2003;65:45-79

11. Levy D, Garrison RJ, Savage DD, Kannel WB, Castelli WP. Prognostic implications of echocardiographically determined left ventricular mass in the framingham heart study. The New England journal of medicine. 1990;322:1561-1566

12. Mathew J, Sleight P, Lonn E, Johnstone D, Pogue J, Yi Q, Bosch J, Sussex B, Probstfield J, Yusuf S. Reduction of cardiovascular risk by regression of electrocardiographic markers of left ventricular hypertrophy by the angiotensin-converting enzyme inhibitor ramipril. Circulation. 2001;104:1615-1621

13. Heineke J, Molkentin JD. Regulation of cardiac hypertrophy by intracellular signalling pathways. Nature reviews. Molecular cell biology. 2006;7:589-600

14. Kakkar R, Lee RT. Intramyocardial fibroblast myocyte communication. Circulation research. 2010;106:47-57

15. Coles B, Fielding CA, Rose-John S, Scheller J, Jones SA, O'Donnell VB. Classic interleukin-6 receptor signaling and interleukin- 6 trans-signaling differentially control angiotensin ii-dependent hypertension, cardiac signal transducer and activator of transcription-3 activation, and vascular hypertrophy in vivo. The American journal of pathology. 2007; 171:315-325

16. Hirota H, Yoshida K, Kishimoto T, Taga T. Continuous activation of gp130, a signal-transducing receptor component for interleukin 6-related cytokines, causes myocardial hypertrophy in mice. Proceedings of the National Academy of Sciences of the United States of America. 1995;92:4862-4866

17. Zhu Y, Li T, Song J, Liu C, Hu Y, Que L, Ha T, Kelley J, Chen Q, Li C, Li Y. The tir/bb-loop mimetic as-1 prevents cardiac hypertrophy by inhibiting il-1r-mediated myd88-dependent signaling. Basic research in cardiology. 2011;106:787-799 
18. Freund C, Schmidt-Ullrich R, Baurand A, Dunger S, Schneider W, Loser P, El-Jamali A, Dietz R, Scheidereit C, Bergmann MW. Requirement of nuclear factor-kappab in angiotensin ii- and isoproterenol-induced cardiac hypertrophy in vivo. Circulation. 2005;111:2319-2325

19. Damilano F, Franco I, Perrino C, Schaefer K, Azzolino O, Carnevale D, Cifelli G, Carullo P, Ragona R, Ghigo A, Perino A, Lembo G, Hirsch E. Distinct effects of leukocyte and cardiac phosphoinositide 3-kinase gamma activity in pressure overload-induced cardiac failure. Circulation. 2011;123:391-399

20. Haghikia A, Stapel B, Hoch M, Hilfiker-Kleiner D. Stat3 and cardiac remodeling. Heart failure reviews. 2011;16:35-47

21. Kvakan H, Kleinewietfeld M, Qadri F, Park JK, Fischer R, Schwarz I, Rahn HP, Plehm R, Wellner M, Elitok S, Gratze P, Dechend R, Luft FC, Muller DN. Regulatory t cells ameliorate angiotensin ii-induced cardiac damage. Circulation. 2009;119:2904-2912

22. Mann DL. Inflammatory mediators and the failing heart: Past, present, and the foreseeable future. Circulation research. 2002;91:988-998

23. Benedict CR, Weiner DH, Johnstone DE, Bourassa MG, Ghali JK, Nicklas J, Kirlin P, Greenberg B, Quinones MA, Yusuf S. Comparative neurohormonal responses in patients with preserved and impaired left ventricular ejection fraction: Results of the studies of left ventricular dysfunction (solvd) registry. The solvd investigators. Journal of the American College of Cardiology. 1993;22:146A-153A

24. Deswal A, Petersen NJ, Feldman AM, Young JB, White BG, Mann DL. Cytokines and cytokine receptors in advanced heart failure: An analysis of the cytokine database from the vesnarinone trial (vest). Circulation. 2001;103:2055-2059

25. da Costa Martins PA, Bourajjaj M, Gladka M, Kortland M, van Oort RJ, Pinto YM, Molkentin JD, De Windt LJ. Conditional dicer gene deletion in the postnatal myocardium provokes spontaneous cardiac remodeling. Circulation. 2008;118:1567-1576

26. Zelarayan L, Renger A, Noack C, Zafiriou MP, Gehrke C, van der Nagel R, Dietz R, de Windt L, Bergmann MW. Nf-kappab activation is required for adaptive cardiac hypertrophy. Cardiovascular research. 2009;84:416-424

27. Hilfiker-Kleiner D, Shukla P, Klein G, Schaefer A, Stapel B, Hoch M, Muller W, Scherr M, Theilmeier G, Ernst M, Hilfiker A, Drexler H. Continuous glycoprotein-130-mediated signal transducer and activator of transcription-3 activation promotes inflammation, left ventricular rupture, and adverse outcome in subacute myocardial infarction. Circulation. 2010;122:145-155

28. Pu WT, Ma Q, Izumo S. Nfat transcription factors are critical survival factors that inhibit cardiomyocyte apoptosis during phenylephrine stimulation in vitro. Circulation research. 2003;92:725-731

29. Bourajjaj M, Armand AS, da Costa Martins PA, Weijts B, van der Nagel R, Heeneman S, Wehrens XH, De Windt LJ. Nfatc2 is a necessary mediator of calcineurin-dependent cardiac hypertrophy and heart failure. The Journal of biological chemistry. 2008;283:22295-22303

30. Sagar S, Liu PP, Cooper LT, Jr. Myocarditis. Lancet. 2011

31. Mason JW, O'Connell JB, Herskowitz A, Rose NR, McManus BM, Billingham ME, Moon TE. A clinical trial of immunosuppressive therapy for myocarditis. The myocarditis treatment trial investigators. The New England journal of medicine. 1995;333:269-275

32. Frustaci A, Chimenti C, Calabrese F, Pieroni M, Thiene G, Maseri A. Immunosuppressive therapy for active lymphocytic myocarditis: Virological and immunologic profile of responders versus nonresponders. Circulation. 2003; 107:857-863

33. Frustaci A, Russo MA, Chimenti C. Randomized study on the efficacy of immunosuppressive therapy in patients with virus-negative inflammatory cardiomyopathy: The timic study. European heart journal. 2009;30:1995-2002

34. Blauwet LA, Cooper LT. Myocarditis. Progress in cardiovascular diseases. 2010;52:274-288

35. Riad A, Westermann D, Zietsch C, Savvatis K, Becher PM, Bereswill S, Heimesaat MM, Lettau O, Lassner D, Dorner A, Poller W, Busch M, Felix SB, Schultheiss HP, Tschope C. Trif is a critical survival factor in viral cardiomyopathy. Journal of immunology. 2011;186:2561-2570

36. Fairweather D, Yusung S, Frisancho S, Barrett M, Gatewood S, Steele R, Rose NR. II-12 receptor beta 1 and toll-like receptor 4 increase il-1 beta- and il-18-associated myocarditis and coxsackievirus replication. Journal of immunology. 2003;170:4731-4737 
37. Riad A, Westermann D, Escher F, Becher PM, Savvatis K, Lettau O, Heimesaat MM, Bereswill S, Volk HD, Schultheiss HP, Tschope C. Myeloid differentiation factor-88 contributes to tlr9-mediated modulation of acute coxsackievirus b3-induced myocarditis in vivo. American journal of physiology. Heart and circulatory physiology. 2010;298:H2024-2031

38. Li K, Xu W, Guo Q, Jiang Z, Wang P, Yue Y, Xiong S. Differential macrophage polarization in male and female balb/c mice infected with coxsackievirus b3 defines susceptibility to viral myocarditis. Circulation research. 2009;105:353-364

39. Shi Y, Fukuoka M, Li G, Liu Y, Chen M, Konviser M, Chen X, Opavsky MA, Liu PP. Regulatory t cells protect mice against coxsackievirus-induced myocarditis through the transforming growth factor beta-coxsackieadenovirus receptor pathway. Circulation. 2010;121:2624-2634

40. Krutzfeldt J, Rajewsky N, Braich R, Rajeev KG, Tuschl T, Manoharan M, Stoffel M. Silencing of micrornas in vivo with 'antagomirs'. Nature. 2005;438:685-689

41. Lanford RE, Hildebrandt-Eriksen ES, Petri A, Persson R, Lindow M, Munk ME, Kauppinen S, Orum H. Therapeutic silencing of microrna-122 in primates with chronic hepatitis c virus infection. Science. 2010;327:198-201

42. Rayner KJ, Esau CC, Hussain FN, McDaniel AL, Marshall SM, van Gils JM, Ray TD, Sheedy FJ, Goedeke L, Liu X, Khatsenko OG, Kaimal V, Lees CJ, Fernandez-Hernando C, Fisher EA, Temel RE, Moore KJ. Inhibition of mir-33a/b in non-human primates raises plasma hdl and lowers vldl triglycerides. Nature. 2011;478:404-407

43. Brenner JL, Jasiewicz KL, Fahley AF, Kemp BJ, Abbott AL. Loss of individual micrornas causes mutant phenotypes in sensitized genetic backgrounds in C. Elegans. Current biology : CB. 2010;20:1321-1325

44. Guo H, Ingolia NT, Weissman JS, Bartel DP. Mammalian micrornas predominantly act to decrease target mrna levels. Nature. 2010;466:835-840

45. O'Connell RM, Kahn D, Gibson WS, Round JL, Scholz RL, Chaudhuri AA, Kahn ME, Rao DS, Baltimore D. Microrna-155 promotes autoimmune inflammation by enhancing inflammatory t cell development. Immunity. 2010;33:607-619

46. Kurowska-Stolarska M, Alivernini S, Ballantine LE, Asquith DL, Millar NL, Gilchrist DS, Reilly J, lerna M, Fraser AR, Stolarski B, McSharry C, Hueber AJ, Baxter D, Hunter J, Gay S, Liew FY, Mclnnes IB. Microrna-155 as a proinflammatory regulator in clinical and experimental arthritis. Proceedings of the National Academy of Sciences of the United States of America. 2011;108:11193-11198

47. Zhu N, Zhang D, Chen S, Liu X, Lin L, Huang X, Guo Z, Liu J, Wang Y, Yuan W, Qin Y. Endothelial enriched micrornas regulate angiotensin ii-induced endothelial inflammation and migration. Atherosclerosis. 2011;215:286-293

48. Kuhl U, Pauschinger M, Schwimmbeck PL, Seeberg B, Lober C, Noutsias M, Poller W, Schultheiss HP. Interferon-beta treatment eliminates cardiotropic viruses and improves left ventricular function in patients with myocardial persistence of viral genomes and left ventricular dysfunction. Circulation. 2003;107:27932798

49. Grun S, Schumm J, Greulich S, Wagner A, Schneider S, Bruder O, Kispert EM, Hill S, Ong P, Klingel K, Kandolf R, Sechtem U, Mahrholdt H. Long-term follow-up of biopsy-proven viral myocarditis: Predictors of mortality and incomplete recovery. Journal of the American College of Cardiology. 2012

50. Duttagupta R, Jiang R, Gollub J, Getts RC, Jones KW. Impact of cellular mirnas on circulating mirna biomarker signatures. PLoS ONE. 2011;6:e20769

51. Pritchard CC, Kroh E, Wood B, Arroyo JD, Dougherty KJ, Miyaji MM, Tait JF, Tewari M. Blood cell origin of circulating micrornas: A cautionary note for cancer biomarker studies. Cancer prevention research. 2012;5:492-497

52. Adachi T, Nakanishi M, Otsuka Y, Nishimura K, Hirokawa G, Goto Y, Nonogi H, Iwai N. Plasma microrna 499 as a biomarker of acute myocardial infarction. Clin Chem. 2010;56:p1-p3

53. Wang GK, Zhu JQ, Zhang JT, Li Q, Li Y, He J, Qin YW, Jing Q. Circulating microrna: A novel potential biomarker for early diagnosis of acute myocardial infarction in humans. Eur Heart J. 2010;31:659-666

54. D'Alessandra Y, Devanna P, Limana F, Straino S, Di Carlo A, Brambilla PG, Rubino M, Carena MC, Spazzafumo L, De Simone M, Micheli B, Biglioli P, Achilli F, Martelli F, Maggiolini S, Marenzi G, Pompilio G, Capogrossi MC. Circulating micrornas are new and sensitive biomarkers of myocardial infarction. European heart journal. 2010;31:2765-2773 
55. De Rosa S, Fichtlscherer S, Lehmann R, Assmus B, Dimmeler S, Zeiher AM. Transcoronary concentration gradients of circulating micrornas. Circulation. 2011;124:1936-1944

56. Widera C, Gupta SK, Lorenzen JM, Bang C, Bauersachs J, Bethmann K, Kempf T, Wollert KC, Thum T. Diagnostic and prognostic impact of six circulating micrornas in acute coronary syndrome. Journal of molecular and cellular cardiology. 2011;51:872-875

57. Morrow DA, de Lemos JA. Benchmarks for the assessment of novel cardiovascular biomarkers. Circulation. 2007;115:949-952

58. Anderson JL, Adams CD, Antman EM, Bridges CR, Califf RM, Casey DE, Jr., Chavey WE, 2nd, Fesmire FM, Hochman JS, Levin TN, Lincoff AM, Peterson ED, Theroux P, Wenger NK, Wright RS, Smith SC, Jr., Jacobs AK, Halperin JL, Hunt SA, Krumholz HM, Kushner FG, Lytle BW, Nishimura R, Ornato JP, Page RL, Riegel B. Acc/aha 2007 guidelines for the management of patients with unstable angina/non st-elevation myocardial infarction: A report of the american college of cardiology/american heart association task force on practice guidelines (writing committee to revise the 2002 guidelines for the management of patients with unstable angina/non st-elevation myocardial infarction): Developed in collaboration with the american college of emergency physicians, the society for cardiovascular angiography and interventions, and the society of thoracic surgeons: Endorsed by the american association of cardiovascular and pulmonary rehabilitation and the society for academic emergency medicine. Circulation. 2007;116:e148-304

59. Reichlin T, Hochholzer W, Bassetti S, Steuer S, Stelzig C, Hartwiger S, Biedert S, Schaub N, Buerge C, Potocki M, Noveanu M, Breidthardt T, Twerenbold R, Winkler K, Bingisser R, Mueller C. Early diagnosis of myocardial infarction with sensitive cardiac troponin assays. The New England journal of medicine. 2009;361:858-867

60. Keller T, Zeller T, Peetz D, Tzikas S, Roth A, Czyz E, Bickel C, Baldus S, Warnholtz A, Frohlich M, Sinning CR, Eleftheriadis MS, Wild PS, Schnabel RB, Lubos E, Jachmann N, Genth-Zotz S, Post F, Nicaud V, Tiret L, Lackner KJ, Munzel TF, Blankenberg S. Sensitive troponin i assay in early diagnosis of acute myocardial infarction. The New England journal of medicine. 2009;361:868-877

61. Devaux Y, Vausort M, Goretti E, Nazarov PV, Azuaje F, Gilson G, Corsten MF, Schroen B, Lair ML, Heymans S, Wagner DR. Use of circulating micrornas to diagnose acute myocardial infarction. Clinical chemistry. 2012;58:559-567

62. Fichtlscherer S, Zeiher AM, Dimmeler S. Circulating micrornas: Biomarkers or mediators of cardiovascular diseases? Arteriosclerosis, thrombosis, and vascular biology. 2011;31:2383-2390

63. Creemers EE, Tijsen AJ, Pinto YM. Circulating micrornas: Novel biomarkers and extracellular communicators in cardiovascular disease? Circulation research. 2012;110:483-495

64. Zampetaki A, Kiechl S, Drozdov I, Willeit P, Mayr U, Prokopi M, Mayr A, Weger S, Oberhollenzer F, Bonora E, Shah A, Willeit J, Mayr M. Plasma microrna profiling reveals loss of endothelial mir-126 and other micrornas in type 2 diabetes. Circulation research. 2010;107:810-817

65. Wang K, Zhang S, Marzolf B, Troisch P, Brightman A, Hu Z, Hood LE, Galas DJ. Circulating micrornas, potential biomarkers for drug-induced liver injury. Proc Natl Acad Sci U S A. 2009;106:4402-4407

66. Arroyo JD, Chevillet JR, Kroh EM, Ruf IK, Pritchard CC, Gibson DF, Mitchell PS, Bennett CF, PogosovaAgadjanyan EL, Stirewalt DL, Tait JF, Tewari M. Argonaute2 complexes carry a population of circulating micrornas independent of vesicles in human plasma. Proceedings of the National Academy of Sciences of the United States of America. 2011;108:5003-5008

67. Valadi H, Ekstrom K, Bossios A, Sjostrand M, Lee JJ, Lotvall JO. Exosome-mediated transfer of mrnas and micrornas is a novel mechanism of genetic exchange between cells. Nature cell biology. 2007;9:654-659

68. Pigati L, Yaddanapudi SC, Iyengar R, Kim DJ, Hearn SA, Danforth D, Hastings ML, Duelli DM. Selective release of microrna species from normal and malignant mammary epithelial cells. PLoS ONE. 2010;5:e13515

69. Turchinovich A, Weiz L, Langheinz A, Burwinkel B. Characterization of extracellular circulating microrna. Nucleic acids research. 2011;39:7223-7233

70. Skog J, Wurdinger T, van Rijn S, Meijer DH, Gainche L, Sena-Esteves M, Curry WT, Jr., Carter BS, Krichevsky AM, Breakefield XO. Glioblastoma microvesicles transport rna and proteins that promote tumour growth and provide diagnostic biomarkers. Nat Cell Biol. 2008;10:1470-1476 
71. Zernecke A, Bidzhekov K, Noels H, Shagdarsuren E, Gan L, Denecke B, Hristov M, Koppel T, Jahantigh MN, Lutgens E, Wang S, Olson EN, Schober A, Weber C. Delivery of microrna-126 by apoptotic bodies induces cxcl12-dependent vascular protection. Science signaling. 2009;2:ra81

72. Vickers KC, Palmisano BT, Shoucri BM, Shamburek RD, Remaley AT. Micrornas are transported in plasma and delivered to recipient cells by high-density lipoproteins. Nature cell biology. 2011;13:423-433

73. Zhang Y, Liu D, Chen X, Li J, Li L, Bian Z, Sun F, Lu J, Yin Y, Cai X, Sun Q, Wang K, Ba Y, Wang Q, Wang D, Yang J, Liu P, Xu T, Yan Q, Zhang J, Zen K, Zhang CY. Secreted monocytic mir-150 enhances targeted endothelial cell migration. Molecular cell. 2010;39:133-144

74. Elmen J, Lindow M, Schutz S, Lawrence M, Petri A, Obad S, Lindholm M, Hedtjarn M, Hansen HF, Berger U, Gullans S, Kearney P, Sarnow P, Straarup EM, Kauppinen S. Lna-mediated microrna silencing in non-human primates. Nature. 2008;452:896-899 

Summary

Samenvatting 


\section{Summary}

Heart failure is associated with both structural and molecular changes in the heart. Cardiac cells respond to stress through the dynamic production of signaling molecules that direct cell behavior. While some of these signaling molecules are adaptive and protect against the development of heart failure, others have frank deleterious effects. The research field of molecular cardiology aims to identify adaptive and maladaptive molecules as targets for clinical therapies and diagnostics. An important and relatively novel class of signaling molecules is formed by the microRNAs. The human genome encodes approximately one thousand microRNAs that govern virtually all cellular processes by binding to messenger RNA and repressing the expression of target proteins. Each microRNA has a unique set of targets that can include hundreds of proteins. MicroRNAs are important for normal bodily functions, but additionally play important roles during a variety of human diseases, including cardiovascular diseases and cancer. In this thesis, we have identified microRNA functions in cardiac inflammation and the development of heart failure, as well as the possibility of using microRNAs in circulating blood as biomarkers for heart disease.

To study dysregulated microRNAs in cardiac inflammation we first profiled cardiac microRNA changes during viral myocarditis. Viral myocarditis is an inflammatory disease of the heart that is often caused by infection with common viruses. Although these viruses directly damage cardiac cells, the immune response significantly contributes to cardiac injury and heart failure development. A large number of microRNAs is up or downregulated in the hearts of both patients and mice suffering from viral myocarditis. We identified patterns of microRNA changes associated with acute inflammation, as well as with disease susceptibility and selected strikingly dysregulated microRNAs for further investigation in a mouse model of viral myocarditis. MicroRNA-155 expression strongly increases in human and mouse myocarditis hearts. Inhibition of microRNA-155 during viral infection reduces immune infiltration and activation, attenuates necrosis in the acute phase and improves survival and cardiac function in mice. In contrast, inhibitors against microRNA-221 and microRNA-222, two highly related microRNA family members that are upregulated in mouse myocarditis, enhance viral replication and aggravate cardiac inflammation and injury. Our results indicate that microRNA changes in the heart during myocarditis include adaptive and maladaptive responses and that inhibiting microRNA-155, or conversely supporting microRNA-221-222, may benefit patients.

Next, we studied the importance of microRNA-155 for the development of heart failure during pressure overload, a model roughly equivalent to arterial hypertension in humans. Mice that lack the microRNA-155 gene exhibit a reduced influx of inflammatory cells in the heart and this is associated with protection from cardiac hypertrophy and contractile dysfunction. Mechanistically, activated inflammatory cells secrete cytokines that contribute to cardiac hypertrophy. Secretion of these factors is promoted by microRNA-155 and is reduced in immune cells of microRNA-155 knockout mice. MicroRNA-155 is 
therefore a maladaptive mediator of inflammation and heart failure during pressure overload and a potential therapeutic target in hypertensive heart disease.

MicroRNAs are abundant in living cells, but can also be detected as freely circulating in human plasma. To study whether such plasma measurements can be used for the diagnosis of cardiovascular disease, we measured microRNA levels in the plasma of patients with varying cardiovascular disorders. Plasma levels of microRNA-208b and microRNA-499, two microRNAs that are enriched in cardiomyocytes, sharply increase during acute myocardial infarction and acute viral myocarditis. Their levels correlate strongly with markers of cardiomyocyte necrosis and are not affected by clinical characteristics such as age, gender, body-mass index, blood pressure, and kidney function. Our data demonstrate the feasibility of using myocyte-enriched microRNAs as biomarkers for cardiac injury during cardiovascular disease in patients.

In summary, this thesis presents important roles for microRNAs during inflammation in hypertrophic and inflammatory heart disease, as well as their use as biomarkers. The studies presented provide insight into the contribution of inflammatory signaling to heart failure. In addition, the identification of individual microRNAs as potential diagnostic and therapeutic targets contributes to the translation of basic microRNA research into clinical applications for cardiovascular disease. 


\section{Samenvatting}

Bij hartfalen treden zowel structurele als moleculaire veranderingen in het hart op. Cellen in het hart reageren op uiteenlopende soorten stress door de dynamische productie van signaalmoleculen welke de werking van de cel beïnvloeden. Sommige van deze signaalmoleculen zijn adaptief en beschermen tegen het ontstaan van hartfalen, terwijl anderen juist overwegend schadelijke effecten hebben op het hart. In de moleculaire cardiologie proberen onderzoekers zulke adaptieve en maladaptieve moleculen te identificeren als aangrijpingspunten voor nieuwe behandelingsmethoden en diagnostiek bij patiënten. Een belangrijke en relatief nieuwe klasse signaalmoleculen zijn de microRNAs. Het totaal menselijke DNA, ofwel genoom, bevat bouwtekeningen voor ongeveer een duizendtal microRNAs. Deze microRNAs beïnvloeden vrijwel alle cellulaire processen door binding aan specifieke 'target' messenger RNAs, hetgeen leidt tot remming van target eiwit productie. Elk microRNA heeft een unieke set aan targets die wel honderden eiwitten kan omvatten. MicroRNAs zijn belangrijk voor normale lichaamsfuncties, maar spelen ook een belangrijke rol bij een veelvoud aan menselijke ziekten, zoals hart- en vaatziekten en kanker. In dit proefschrift hebben we enerzijds microRNAs geïdentificeerd welke betrokken zijn bij ontsteking in het hart en hartfalen, en anderzijds de mogelijkheid onderzocht om vrij circulerende microRNAs in het bloed te gebruiken als biomarkers voor hartziekten.

Om microRNAs met een rol in cardiale ontsteking te identificeren hebben we eerst een expressie 'profiling' uitgevoerd van alle microRNAs in het hart tijdens virale myocarditis. Virale myocarditis is een ontsteking van het hart die wordt veroorzaakt door infectie van het hart met veelvoorkomende virussen. Hoewel deze virussen directe hartschade kunnen veroorzaken, draagt de immuunrespons in belangrijke mate bij aan de onomkeerbare hartbeschadiging en daaruit volgend hartfalen. In de harten van patiënten en muizen welke lijden aan virale myocarditis is de concentratie van een groot aantal microRNAs, vergeleken met gezonde controles, verhoogd of juist verlaagd. Op deze wijze konden we microRNA patronen identificeren welke geassocieerd zijn met acute ontsteking of gevoeligheid voor de ziekte. Vervolgens hebben we microRNAs met een opvallend expressiepatroon tijdens virale myocarditis geselecteerd voor andere bestudering in een muizenmodel van deze ziekte. De expressie van microRNA-155 in het hart neemt sterkt toe tijdens virale myocarditis in zowel mensen als muizen. Remming van microRNA-155 activiteit tijdens virale infectie remt de immuunrespons in het hart, vermindert necrose van hartspiercellen tijdens de acute ziektefase en verbetert de overleving en hartfunctie in muizen. Remming van de twee sterk aan elkaar verwante microRNAs-221 en -222, welke ook verhoogd tot expressie komen tijdens virale myocarditis in de muis, leidt daarentegen tot toegenomen virusvermenigvuldiging, ontsteking en schade aan het hart. Deze bevindingen laten zien dat microRNA veranderingen in het hart tijdens virale myocarditis zowel adaptieve als maladaptieve veranderingen omvatten en dat remming van miR-155, of juist stimulering van microRNA-221 en -222, gunstig zou kunnen zijn voor patiënten met deze ziekte. 
Vervolgens hebben we de effecten van microRNA-155 bestudeerd tijdens de ontwikkeling van hartfalen door drukoverbelasting in muizen, middels een diermodel dat vergelijkbaar is met arteriële hypertensie bij mensen. Muizen welke het microRNA-155 gen missen (microRNA-155 knockout muizen) vertonen bij drukoverbelasting een verminderde infiltratie van ontstekingscellen in het hart en zijn deels beschermd tegen hartspierverdikking en afname van knijpkracht in vergelijking met normale muizen. Geactiveerde ontstekingscellen scheiden stoffen (cytokinen) uit welke bijdragen aan hartspiercelverdikking. Uitscheiding van deze cytokinen wordt gestimuleerd foor microRNA-155 en is verminderd in ontstekingscellen van microRNA-155 knockout muizen. MicroRNA-155 is dus een maladaptieve regulator van ontsteking en hartfalen tijden drukoverbelasting, en vormt een potentieel doelwit voor de behandeling van hypertensieve hartziekten.

MicroRNAs bevinden zich voornamelijk in levende cellen, maar kunnen ook gedetecteerd worden als vrij circulerende deeltjes in het plasma van de menselijke bloedsomloop. Om te bestuderen of zulke plasma bepalingen bruikbaar zijn voor de diagnose van hart- en vaatziekten, hebben we microRNA niveaus gemeten in het plasma van patiënten met uiteenlopende cardiovasculaire aandoeningen. Plasma niveaus van microRNA-208b en microRNA-499, twee microRNAs die sterk geconcentreerd zijn in hartspiercellen, vertonen een sterke stijging tijdens het doormaken van een acute hartinfarct of acute virale myocarditis. Deze plasma niveaus correleren goed met gevestigde markers van hartspiercelverval en worden niet verstoord door patiënt-specifieke factoren zoals leeftijd, geslacht, gewicht, bloeddruk of nierfunctie. Onze data tonen daarmee aan dat het mogelijk is om hartspiercel-microRNAs te gebruiken als plasma biomarkers voor hartschade bij met cardiovasculaire aandoeningen.

Samenvattend presenteert dit proefschrift belangrijke rollen voor microRNAs bij het ontstekingsproces in hypertrofische en inflammatoire hartziekten, en poneert het tevens de mogelijkheid tot het gebruik van microRNAs als biomarkers voor hart- en vaatziekten. De beschreven studies bieden inzicht in de bijdrage van ontsteking aan de ontwikkeling van hartfalen. Daarnaast draagt de identificatie van individuele microRNAs als potentiële doelwitten voor nieuwe diagnostiek en behandelingen bij aan de vertaalslag van basaal microRNA onderzoek naar klinische toepassingen voor de hart- en vaatziekten. 

Nawoord 


\section{Nawoord}

Klaar. Met de proeve van wetenschappelijke bekwaamheid die minstens zozeer doorzettingsvermogen test. Na jaren van veel struikelen en veel opstaan een onwerkelijk maar heerlijk gevoel. Sinds mijn eerste wankele wetenschappelijke stapjes als student in 2004 heeft het onderzoek me veel verscheidenheid gebracht. Van imaging in Maastricht en Irvine naar de microRNAs en oncologie in Boston, om vervolgens via de wondere wereld van targeted delivery-research wederom in Maastricht bij mijn uiteindelijke promotieproject van microRNAs in de cardiologie uit te komen. Een mens heeft wel eens een rechtere lijn getrokken. Desalniettemin heeft de variëteit aan verschillende labs en onderwerpen een weelde aan ervaringen opgeleverd. Ik ben dankbaar voor de bijdrage die velen hieraan geleverd hebben en wil enkele personen in het bijzonder bedanken.

Allereerst mijn promotor. Geachte professor Heymans, beste Stephane, hartelijk dank voor het vertrouwen dat ik de afgelopen drie jaar van je heb ontvangen. Je explosief enthousiasme en optimisme zijn ongeëvenaard en na de brainstorms over mijn projecten heb ik geregeld een extra bakje koffie nodig gehad om mijn gedachten weer in het gareel te krijgen. Juist door onze verschillen in stijl van onderzoek doen en schrijven heb ik veel van je kunnen leren, met name over de balans tussen nuance en leesbaarheid. Ik ben benieuwd welke opportuniteiten zich de komende jaren nog aan je zullen voordoen, maar je mix van Belgische vermomming en Hollandse assertiviteit zullen ongetwijfeld op velerlei plekken tot hun recht komen.

Mijn copromotor Blanche Schroen. Je technische kennis en analytisch vermogen hebben een grote invloed gehad op dit proefschrift. Je was zowel begeleider, klankbord als teamgenoot. Bij ons gezamenlijke puzzelen aan de microRNA projecten in dit boekje hebben we een hoop hoofdbrekens, teleurstellingen en overwinningen gedeeld. Ik wens je alle succes toe met de ontpopping tot zelfstandig PI en met combinatie tussen werk en jullie groeiende gezinnetje.

Mijn paranimfen Bob en Johan. Bob, sinds de brugklas lopen we eigenlijk op bijna alle fronten gelijk op. Des te mooier dat we grofweg gelijktijdig onze promoties af zullen ronden. Je nuchtere humor is het perfecte antigif tegen zowel teleurstellingen als zelfoverschatting (dit in tegenstelling tot je sportkwaliteiten: die werken bij mij enkel tegen zelfoverschatting). Ik ben trots dat je bij mijn verdediging achter me zult staan.

Johan, collega-telg van de MMIT PhD academy. Enthousiaste jonge researchhond, hoogleraar in de gadgetkunde en trouwe karaokepartner 'voor al uw feesten en partijen'. Ik heb genoten van onze tijd als kamergenoten op de 4 e en alles wat daaruit voortgevloeid is. Hopelijk kunnen we je het komend jaar weer op komen zoeken bij je nieuwe researchavontuur in Boston. Mooi dat je er als paranimf bij bent.

En dan natuurlijk het lab zelf. De grillen van een promovendus komen nergens zo prachtig tot uiting als bij de collega's die de hele week naast je staan en zitten. Ons clubje heeft 
inmiddels een aardige kritische massa bereikt maar daarbinnen heeft zo'n beetje iedereen wel bijgedragen aan mijn werkplezier van de afgelopen jaren. Laat ik beginnen met Wouter Verhesen, collega en kamergenoot in mijn laatste jaren. Moleculaire hoeksteen van het lab en uomo universale. Je vele kanten hebben ogenschijnlijk niets met elkaar van doen: wetenschapper, sterrenkijker, stemmings-inverteur, archeoloog, kunstenaar, darter en cartoonist. Je gevoel voor humor en hand van de meester zijn van grote waarde geweest voor zowel het proces als resultaat van deze promotie, waarvoor dank. Rick, nestor en labmanager: door jouw organisatie was werken in ons lab een logistiek feest en ik heb met veel plezier met je samengewerkt. Anna, de beste pretoogjes van het lab en een Brits accent om jaloers op te zijn. Wat je ook zegt, het klinkt altijd goed. Met levenservaring opgedaan in zo'n beetje alle landen van Europa - behalve Luxemburg - raak je je relaxte mojo niet een-twee-drie kwijt en jouw "it'll be fine..." is inmiddels een begrip. Geert van Almen, Eindhovense boy uit het boekje, prins van de koffie en koning van de woordgrap. Jammer dat we maar een jaartje een kamer gedeeld hebben! Gelukkig was dit niet te kort om te genieten van jouw zelf gezette bakkies (the best in town), je muzikale inborst ("ik heb effe underground radio aangezet op JOUW computer, dat is wel ok toch?"), en prachtige verhalen ("ben jij misschien dokter want ik heb hier ECHT last van"). Mooie tijden. Marieke Rienks, of rupsie, of mimelecan: medisch partner in basic crime en trouwe congresgenoot. Hebben samen nog ontdekkingen gedaan, bijvoorbeeld dat er in de buurt van Les Diablerets twee hotel Eurotel Victoria's zijn. We zaten vaak op één lijn qua lab-beleving en dat is in een promotie heel wat waard. Succes met je eigen afronding, ik heb er alle vertrouwen in dat je dat varkentje gaat wassen. Derks, ongepolijste bioloog met de beste T-cel receptor imitatie van ons lab. Je bent zo eigenwijs als nieuwsgierig en enthousiast en van die mix zal je in de wetenschap nog veel plezier beleven. Bedankt voor de gezelligheid. Tim, je hebt een waardevol snufje Deutsche Grundlichkeit aan het lab toegevoegd. Ik ben benieuwd wat er de komende jaren uit jouw koker rolt en wens je alle succes en plezier met het proces. Lucas, Ward en Sophie, kroonprinsen en -prinses van onze Belgische (pardon Vlaamse) dependance. Veel succes met ook jullie laatste loodjes binnenkort. In Leuven ook een bijzonder woord van dank aan Paolo. We hebben samen heel wat uurtjes dierenwerk versleten en je bijdrage aan dit proefschrift is groot geweest. Grazie amico! Georg, bless the Suiss for sending you over to us. I imagine you still have nightmares about my relentless bothering you about the arrays. Sudo sleep well, it is done now! Thanks for everything. Laura, je nuchtere vrolijkheid wordt gemist op het lab. Ik heb met plezier met je gewerkt en ga de uitspraak van nexetrien niet meer vergeten. Verder dank voor een leuke samenwerking aan Mark Schellings, Mark van Bilzen, Sylvia en tot slot onze onmisbare klinische tak: achtereenvolgens Robert, Casper en Hazebroek. De raakvlakken waren met eenieder van jullie verschillend (boksen, muziek en studententijd) maar daarom niet minder goed. Wat Casper en Mark betreft: een man weet pas wattie mist als zijn WESP-student er niet is. Bedankt voor het enthousiasme en het snijden van heel veel coupes. Barbara, rots in de administratieve branding: ik ga je gevoel voor humor missen.

Mijn bazen van het eerste uur, professor Hofstra, Reutelingsperger en Narula! Leo, spin doctor van het MMIT laboratory. Waar jij je hoofd laat zien is het nooit saai. Wat heb je me 
veel uitgedaagd om mijn grenzen te verleggen. Van reviews schrijven ("het is nog niet sexy genoeg..") tot spreken op te grote congressen ("of vind je dat je daar nog te licht voor bent?") en geweldige onderzoeksstages in California en Boston. Het heeft mijn wetenschappelijke honger sterk geprikkeld. Een aantal van je bijzondere kwaliteiten zie ik als voorbeeld, zoals je hypnotiserende sprekerskwaliteiten en denkkracht in de grote lijnen. Selectief niet beantwoorden van mails valt daar overigens niet onder, je capaciteit om daarmee weg te komen dan weer wel. Chris, je was de eerste basaal onderzoeker die ik in het wild tegenkwam. Ik heb genoten van jouw zorgvuldig zoeken naar de juiste onderzoeksvragen en non-template experimenten die deze vragen zouden kunnen beantwoorden. Finally, Jagat, thank you for many stimulating discussions both in Irvine and afterwards. Mijn overige collega's van de MMIT clan. Nicole, jouw vast chirurgische hand is de meest constante factor geweest in de afgelopen jaren. Bedankt voor al je inzet en warme interesse. Maarten Anderegg aka Maar-eleven: een maand lang met twee volwassen kerels in harmonie op dezelfde kamers wonen en werken is niet vanzelfsprekend. Vraag maar aan Makiki. Toch was ons avontuur in Boston een fantastische ervaring waar ik onverwacht een goede vriend aan over gehouden heb. Suus en Reinier, California was niet hetzelfde geweest zonder jullie. Het waren memorabele maanden die ik door de 'casual fridays', goede tripjes en natuurlijk de Cadillac niet snel zal vergeten. Ward, bedankt voor je gezelligheid en mooie lange verhalen. Ik hoop dat promotiegate nog een goed vervolg gaat vinden. Dank ook aan Abdel, Ewald en Bas en aan de biochemische kant Niko, Petra, Lisette, Heidi, Cecile en Kristoff.

Khalid Shah, my mentor in Boston. Dear Khalid, your receiving the Harvard Medical School Young Mentor Award earlier this year comes as no surprise to me. The amount of handson supervision that you invest in your students is amazing. You have taught me the basics of molecular biology, stell cells, gene therapy and of course the psychology of science. Your faith in my capabilities has been a huge stimulus. I look forward to keeping in touch with you. In your lab I would especially like to thank Shawn "living the dream" Hingtgen for a lot of help and a good time.

De open samenwerking tussen labs in het Groot Cardiolab biedt een geweldige omgeving om ieders onderzoek optimaal tot zijn recht te laten komen. Enkelen die hieraan hebben bijgedragen wil ik met name noemen: Leon, Paula, Kanita, Steve, Leonne, Gustavo, Natasja, Monica, Ella, Ellen, en Rio. De kerst- en afscheidsborrels met labdance waren op hun manier uniek. Tenslotte mijn dank aan alle indirecte collega's die verder hebben bijgedragen aan mijn werk de afgelopen jaren. Fazzi, Ben, Agnieszka, Jacques, Helma en Peter van de farmacologie, Nico van de kindergeneeskunde. Bij de moleculaire genetica Lauran, Marion en Menno en zeker ook Guillaume, bij wie ik altijd terecht kon voor een vraag of ontspannend praatje. De medewerkers van het CPV, in het bijzonder Rick en Clarice.

Graag wil ik ook Prof. L. de Windt, Prof. H.J.G.M. Crijns, Prof. M.J. Post, Prof. Y.M. Pinto en Prof. T. Thum bedanken voor het beoordelen van mijn proefschrift. 
Tot slot wil ik graag nog wat veren uitdelen aan degenen die op de achtergrond zo belangrijk zijn geweest. Allereerst mijn vader en moeder, respectievelijk de bèta en alfa in mij, en mijn zusje Ilke. Een vaste basis is onmisbaar voor al dat wil groeien. Als die basis ook nog een warm, veilig en humorvol nest vormt mag de spruit zich gelukkig prijzen. Bij dezen.

Lieve Veer, de laatste loodjes hebben ook van jou offers gevergd. Bedankt voor je onaflatende lieve aandacht, afleiding en ondersteuning. Ik kan met je lachen en op je bouwen. Je bent een levenskunstenares waar ik nog lang van en mee hoop te genieten.

Maarten Corsten,

Maastricht, juni 2012 

Curriculum Vitae List of Publications 


\section{Curriculum Vitae}

Maarten Corsten was born on June $27^{\text {th }} 1983$ in Breda, The Netherlands. After obtaining his gymnasium degree summa cum laude at the Mencia de Mendoza Lyceum in Breda in 2001, he enrolled in his medical studies at Maastricht University that same year. He initiated training in cardiovascular research as a student with Prof. dr. L. Hofstra in 2004, focusing on molecular imaging of cell death. Supported by grants from the Netherlands Heart Foundation and the KWF Cancer Foundation he spent several months in the labs of Prof. dr. J. Narula at the University of Irvine, California in 2005 and of Dr. K. Shah at the Center of Molecular Imaging Research at Massachusetts General Hospital in Boston in 2006, 2008 and 2009. Meanwhile, he completed the first year of Economics at Maastricht University in 2005 and received the Kootstra Talent Fellowship from the Maastricht University Faculty of Medicine in 2007. After receiving his medical degree in 2009, Maarten Corsten commenced his PhD program in the lab of Prof. dr. S. Heymans at the Maastricht University Department of Cardiology, studying the biological importance of microRNAs in inflammatory heart disease. In 2011, he was awarded the Dutch-German Basic Research young investigator award and the runner-up young investigator award at the Heart Failure Association of the European Society of Cardiology. He finished his research in June 2012 and currently works as a resident specializing in Internal Medicine at the Meander Medisch Centrum in Amersfoort. 


\section{List of publications}

Corsten MF, Kietselaer BLJH, Bennaghmouch A, Reutelingsperger CPM, Boersma HH, Hofstra L. A molecular view of apoptosis with annexin a5: A focus on cardiovascular disease. Annexins. 2005;2

Corsten MF, Hofstra L, Narula J, Reutelingsperger CP. Counting heads in the war against cancer: Defining the role of annexin a5 imaging in cancer treatment and surveillance. Cancer Res. 2006;66:1255-1260

Corsten MF, Reutelingsperger CP, Hofstra L. Imaging apoptosis for detecting plaque instability: Rendering death a brighter facade. Current opinion in biotechnology. 2007; 18:83-89

4. Wolters SL, Corsten MF, Reutelingsperger CP, Narula J, Hofstra L. Cardiovascular molecular imaging of apoptosis. European journal of nuclear medicine and molecular imaging. 2007;34 Supp/ 1:S86-98

Corsten MF, Miranda R, Kasmieh R, Krichevsky AM, Weissleder R, Shah K. Microrna-21 knockdown disrupts glioma growth in vivo and displays synergistic cytotoxicity with neural precursor cell delivered s-trail in human gliomas. Cancer Res. 2007;67:8994-9000

Corsten MF, Shah K. Therapeutic stem-cells for cancer treatment: Hopes and hurdles in tactical warfare. Lancet Oncol. 2008;9:376-384

Laufer EM, Winkens HM, Corsten MF, Reutelingsperger CP, Narula J, Hofstra L. Pet and spect imaging of apoptosis in vulnerable atherosclerotic plaques with radiolabeled annexin a5. The quarterly journal of nuclear medicine and molecular imaging : official publication of the Italian Association of Nuclear Medicine. 2009;53:26-34

Corsten MF, Dennert R, Jochems S, Kuznetsova T, Devaux Y, Hofstra L, Wagner DR, Staessen JA, Heymans * $^{*}$, Schroen B* (*contributed equally). Circulating microrna-208b and microrna-499 reflect myocardial damage in cardiovascular disease. Circulation. Cardiovascular genetics. 2010;3:499-506

Devaux Y, Vausort M, Goretti E, Nazarov PV, Azuaje F, Gilson G, Corsten MF, Schroen B, Lair ML, Heymans S, Wagner DR. Use of circulating micrornas to diagnose acute myocardial infarction. Clinical chemistry. 2012;58:559-567

Corsten MF, Schroen B, Heymans S. Inflammation in viral myocarditis: Friend or foe? Trends in molecular medicine. 2012;18:426-437 
Corsten MF, Papageorgiou A, Verhesen W, Carai P, Lindow M, Obad S, Summer G, Coort SL, Hazebroek M, van Leeuwen R, Gijbels MJ, Wijnands E, Biessen EA, De Winther MP, Stassen FR, Carmeliet P, Kauppinen S, Schroen B*, Heymans * $^{*}$ ( contributed equally). Microrna profiling identifies microrna-155 as an adverse mediator of cardiac injury and dysfunction during acute viral myocarditis. Circulation research. 2012;111:415-425

Heymans $S^{*}$, Corsten MF*, Carai P, Verhesen W, Van Leeuwen REW, Stöger L, Wijnands E, Janssen B, Creemers EE, Pinto YM, Zacchigna S, Giacca M, Grimm D, Schürmann N, Vigorito E, Thum T, Stassen F, Carmeliet P, Mayr M, De Windt LJ, Lutgens E, De Winther M, Papageorgiou AP, Schroen B ( ${ }^{*}$ contributed equally). Macrophage microrna-155 controls cardiac hypertrophy and failure. Submitted.

Corsten MF, Papageorgiou A, Verhesen W, Hazebroek M, Carai P, Summer G, Verheyen F, Van Leeuwen REW, Schroen B, Heymans S. The microrna-221-222 family controls viral replication and cardiac inflammation in acute enteroviral myocarditis. In preparation.

\section{Book chapters}

Corsten MF, Bennaghmouch A. Optical characterization of arterial apoptosis. In: Shah K, ed. Molecular imaging - Methods in molecular biology. Humana Press 2011; vol. 680, pp 117-29.

Anderegg MCJ, Corsten MF. MicroRNA adjuvants in stem cell-based cancer therapy. In: Shah K, ed. Stem cell-based therapeutics for cancer. Wiley Blackwell 2012. In press. 